\title{
A Short Total Synthesis of Benzophenanthridine Alkaloids via a Rhodium(III)-Catalyzed C-H Ring-Opening Reaction
}

\author{
Narasingan Aravindan and Masilamani Jeganmohan* \\ Department of chemistry, Indian Institute of Technology Madras, Chennai 600036, Tamil Nadu, \\ India \\ E-mail: mjeganmohan@iitm.ac.in
}

Electronic Supplementary Information

Table of Contents

1. X-Ray analysis

2. Mechanistic Investigation

3. Copies of ${ }^{1} \mathrm{H}$ and ${ }^{13} \mathrm{C}$ NMR
S2-S3

S4

S5-S122 


\section{Crystallographic Data of Compound 3aa}

Table S1. X-Ray Analysis of Compound 3aa

\begin{tabular}{|c|c|}
\hline & $3 \mathbf{a a}$ \\
\hline empirical formula & $\mathrm{C}_{24} \mathrm{H}_{28} \mathrm{~N}_{2} \mathrm{O}_{4}$ \\
\hline formula weight & 408.48 \\
\hline Temp $(\mathrm{K})$ & 296 \\
\hline crystal system & Monoclinic \\
\hline space group & $\mathrm{P} 2{ }_{1} / \mathrm{c}$ \\
\hline $\mathrm{a}(\AA)$ & $8.3634(3)$ \\
\hline $\mathrm{b}(\AA)$ & $13.1676(5)$ \\
\hline c $(\AA)$ & $21.0318(9)$ \\
\hline $\mathrm{a}(\mathrm{deg})$ & 90 \\
\hline $\mathrm{b}(\mathrm{deg})$ & $100.7290(18)$ \\
\hline $\mathrm{g}(\mathrm{deg})$ & 90 \\
\hline Volume & $2275.66(15)$ \\
\hline $\mathrm{Z}$ & 4 \\
\hline$\rho_{\text {calcd }}\left(\mathrm{Mg} \mathrm{m}^{-3}\right)$ & 1.192 \\
\hline$\mu\left(\mathrm{mm}^{-1}\right)$ & 0.081 \\
\hline $\mathrm{F}(000)$ & 872 \\
\hline crystal size (mm) & $0.250 \times 0.220 \times 0.100$ \\
\hline$\Theta$ range $(\mathrm{deg})$ & 1.834 to 24.996. \\
\hline $\begin{array}{l}\text { no. of collected/unique } \\
\text { reflections }\end{array}$ & $16986 / 4007[\mathrm{R}(\mathrm{int})=0.0377]$ \\
\hline $\begin{array}{l}\text { no. of data / restraints / } \\
\text { parameters }\end{array}$ & 4007 / 0 / 281 \\
\hline GOF & 1.015 \\
\hline $\mathrm{R} 1, w \mathrm{R} 2(I>2 \sigma(I))$ & $0.0456,0.1054$ \\
\hline $\mathrm{R} 1, w \mathrm{R} 2$ (all data) & $0.0842,0.1268$ \\
\hline GOF & 1.015 \\
\hline$\Delta \rho_{\max } / \Delta \rho_{\min }\left(\mathrm{e} \AA^{-3}\right)$ & $0.15 /-0.16$ \\
\hline
\end{tabular}




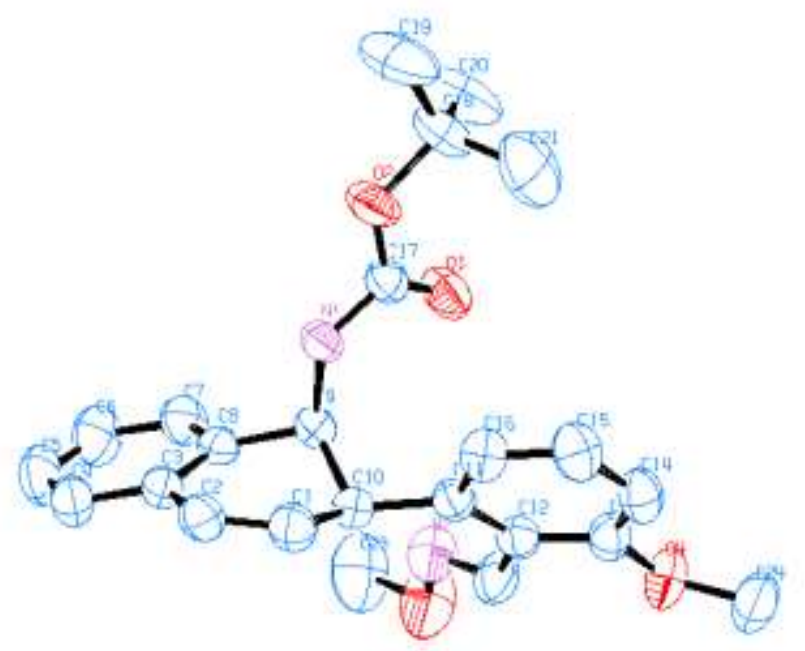

Figure S1: ORTEP representation of compound 3aa displaying thermal elliposoid at 50\% probability. 
${ }^{1} \mathrm{H}$ NMR spectra of compound d-1f $\left(\mathrm{CDCl}_{3}, 400 \mathrm{MHz}\right)$.

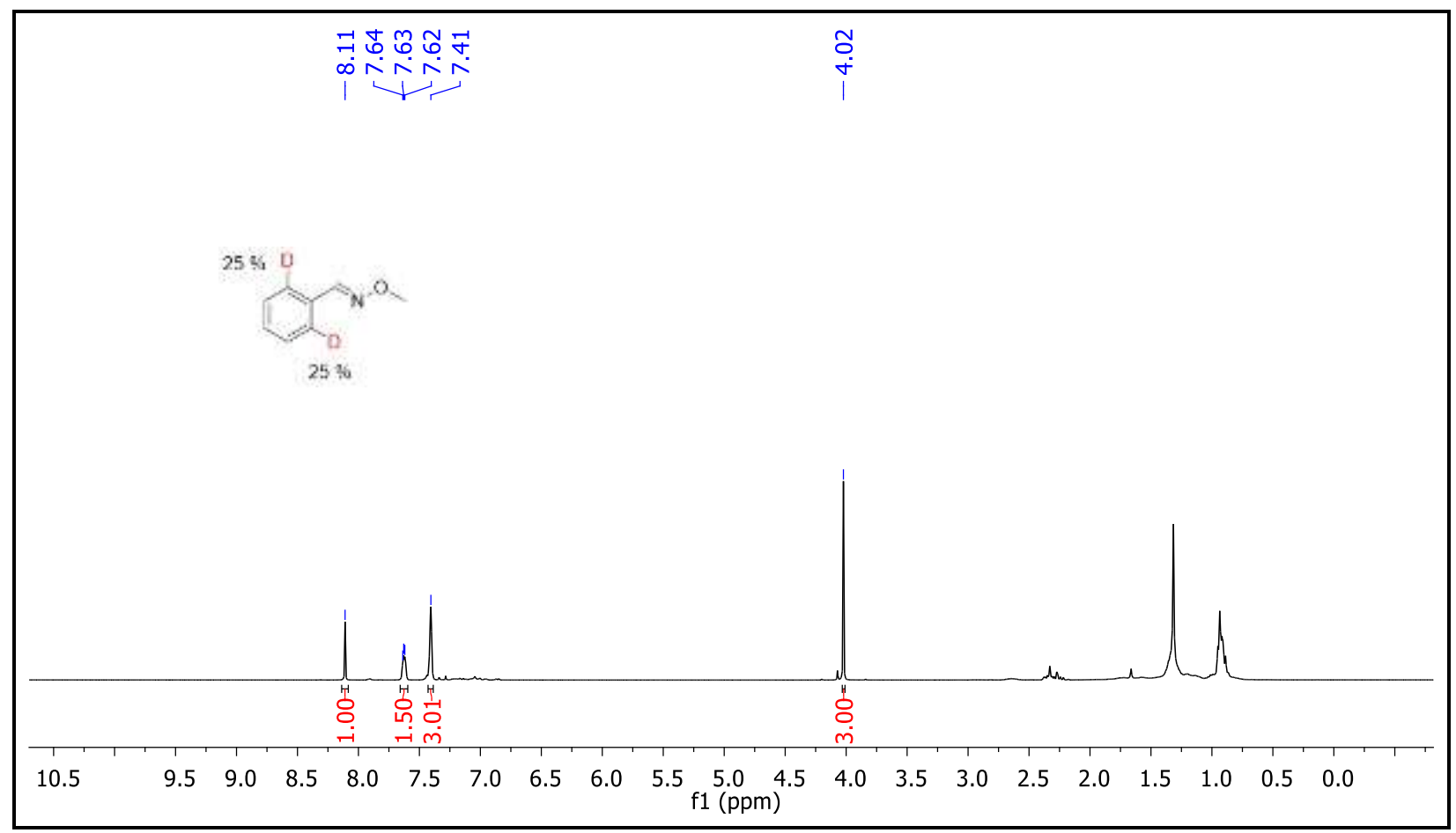

${ }^{1} \mathrm{H}$ NMR spectra of compound d-3fa $\left(\mathrm{CDCl}_{3}, 400 \mathrm{MHz}\right)$.

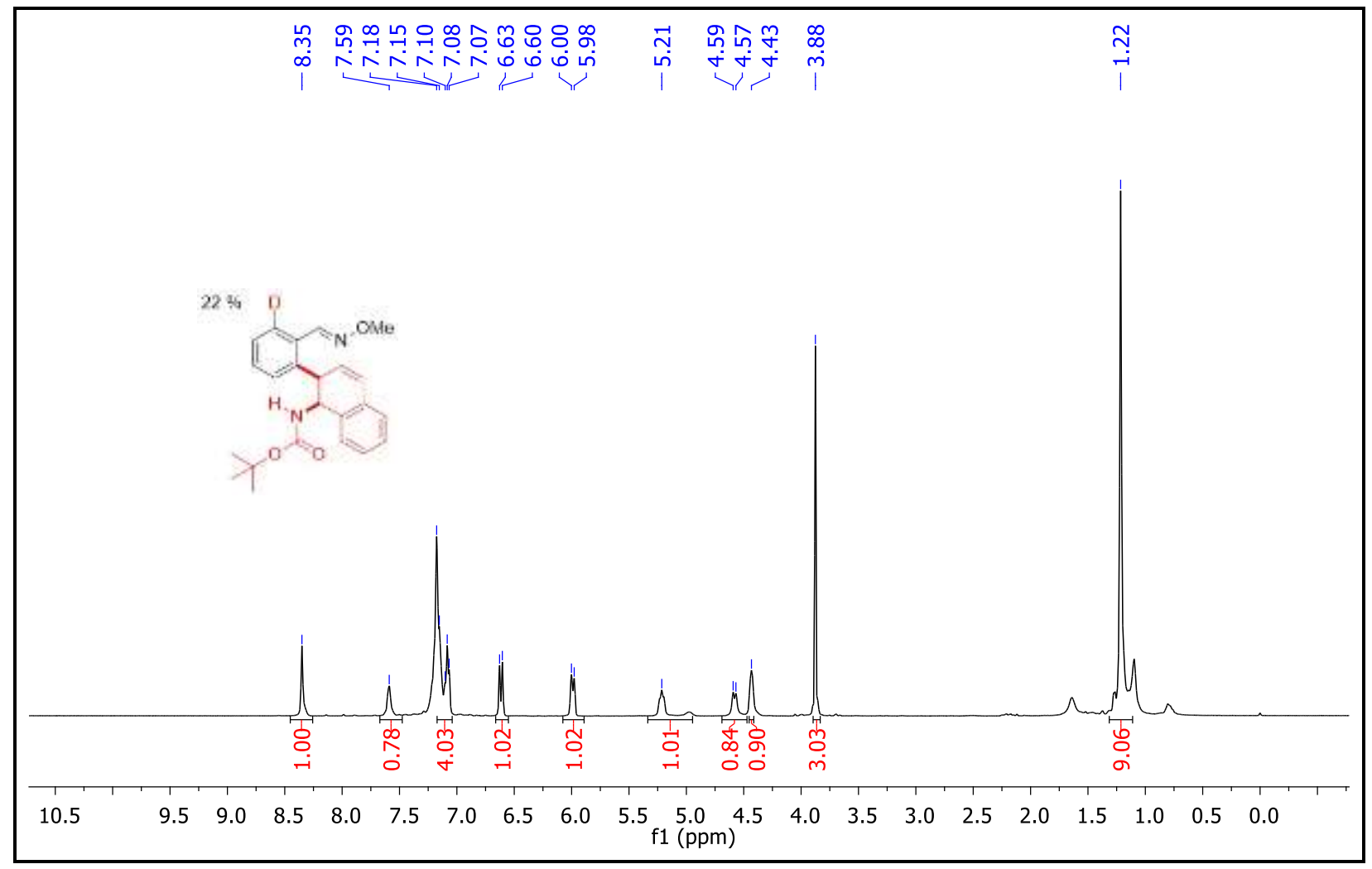


${ }^{1} \mathrm{H}$ and ${ }^{13} \mathrm{C}\left\{{ }^{1} \mathrm{H}\right\}$ NMR Spectra of Compound 3aa $\left(\mathrm{CDCl}_{3}, 400 \mathrm{MHz}\right)$.
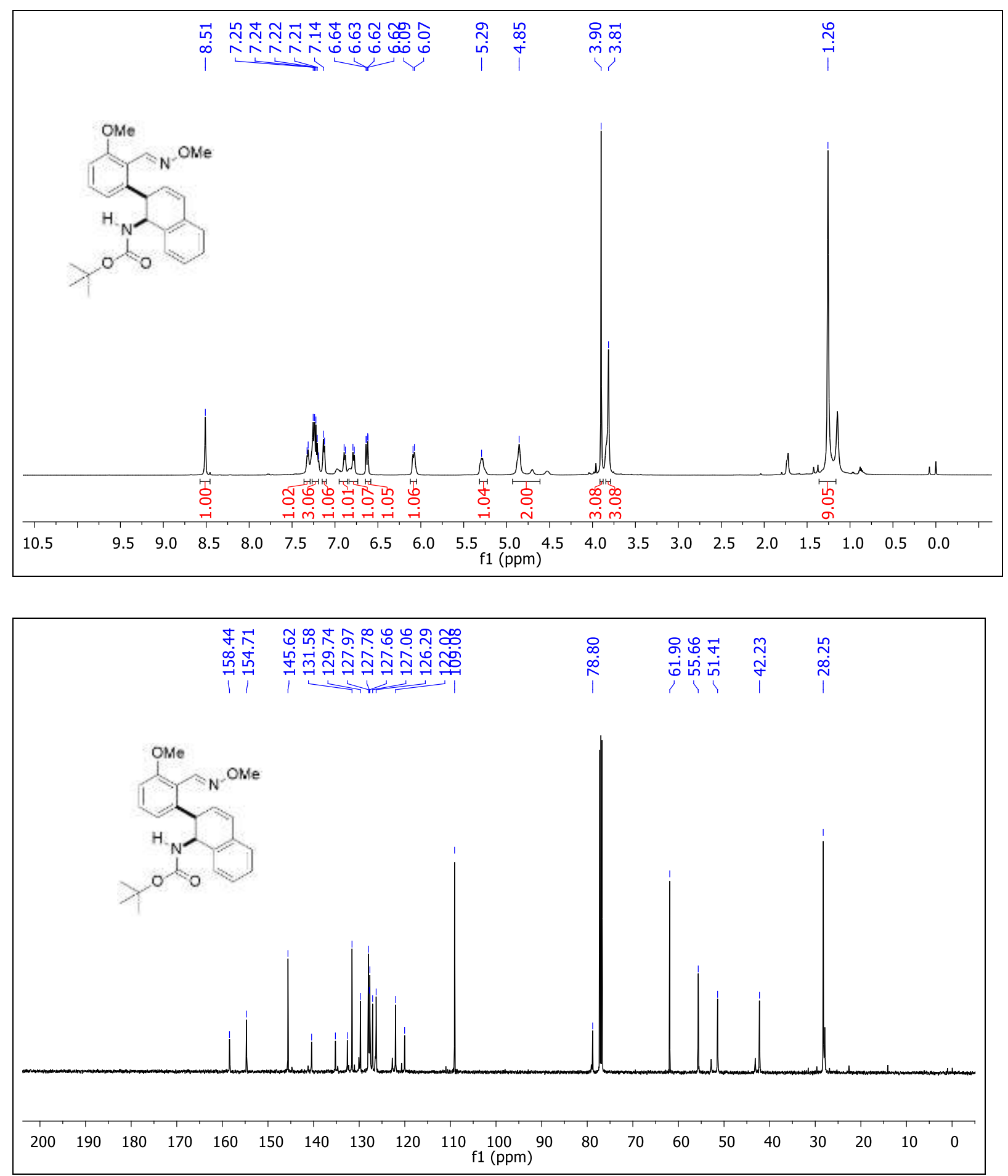
DEPT (135) NMR Spectrum of Compound 3aa $\left(\mathrm{CDCl}_{3}, 101 \mathrm{MHz}\right)$.

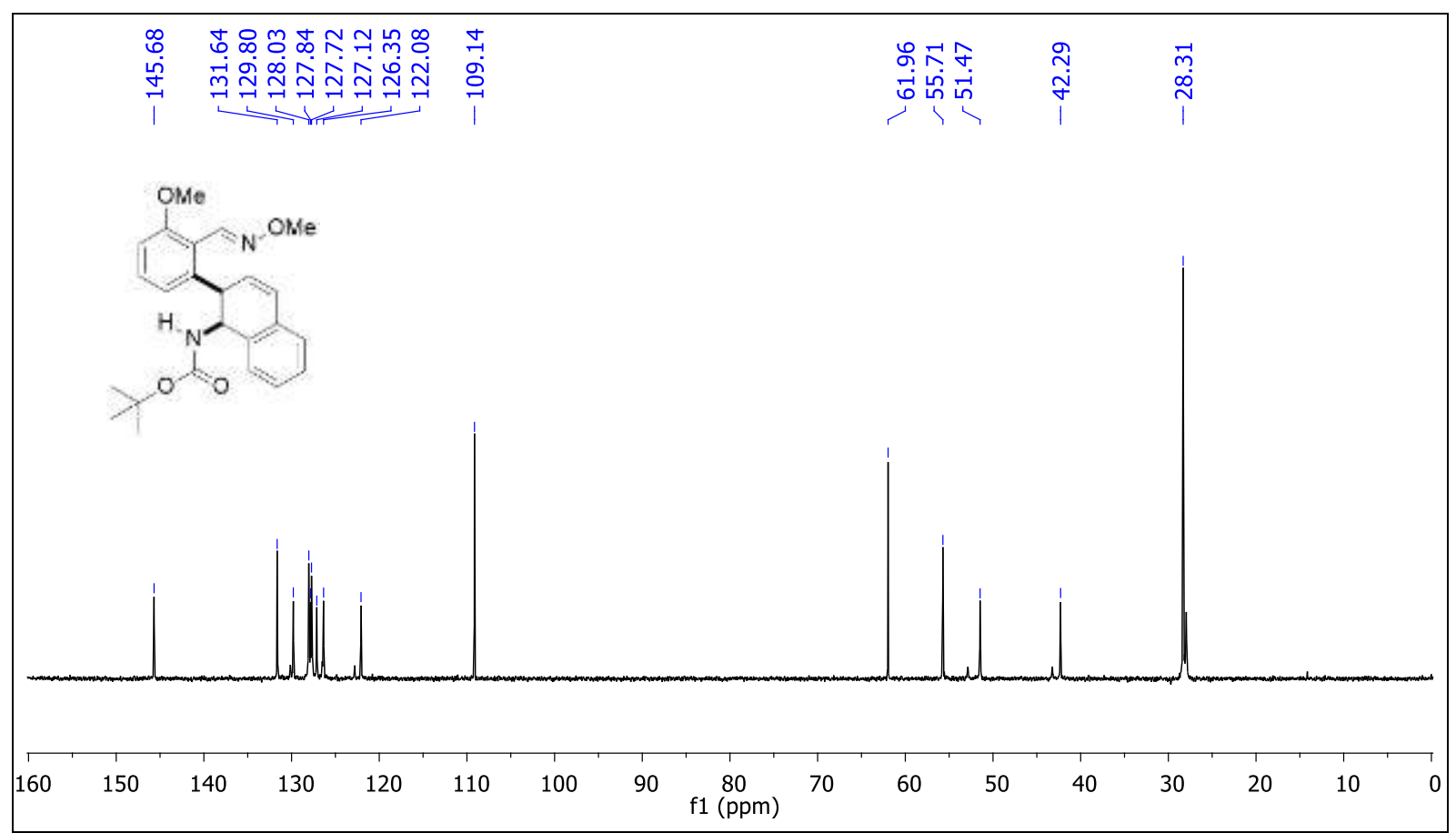


${ }^{1} \mathrm{H}$ and ${ }^{13} \mathrm{C}\left\{{ }^{1} \mathrm{H}\right\}$ NMR Spectra of Compound $3 \mathbf{b a}\left(\mathrm{CDCl}_{3}, 400 \mathrm{MHz}\right)$.
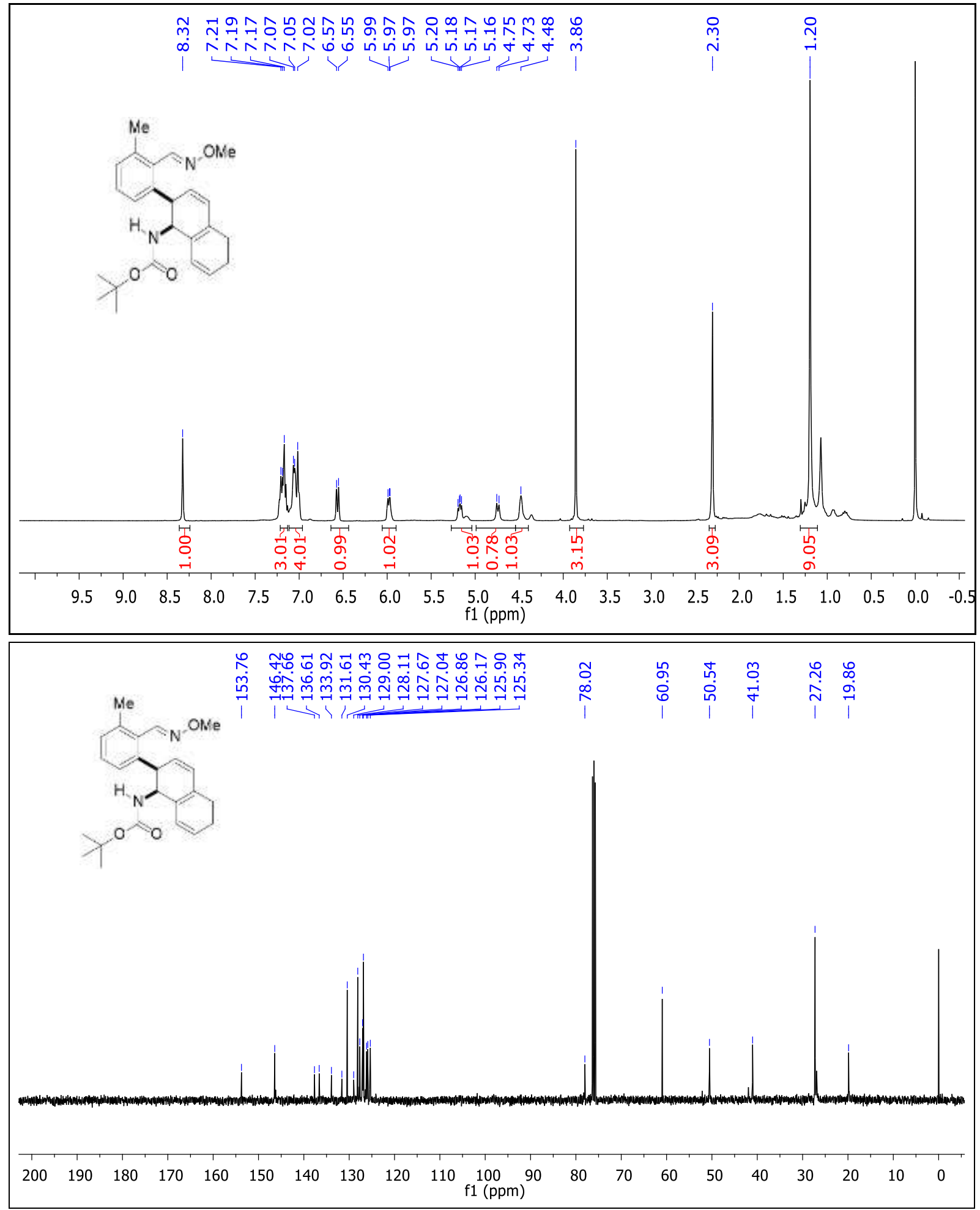
DEPT (135) NMR Spectrum of Compound $3 \mathbf{b a}\left(\mathrm{CDCl}_{3}, 101 \mathrm{MHz}\right)$.

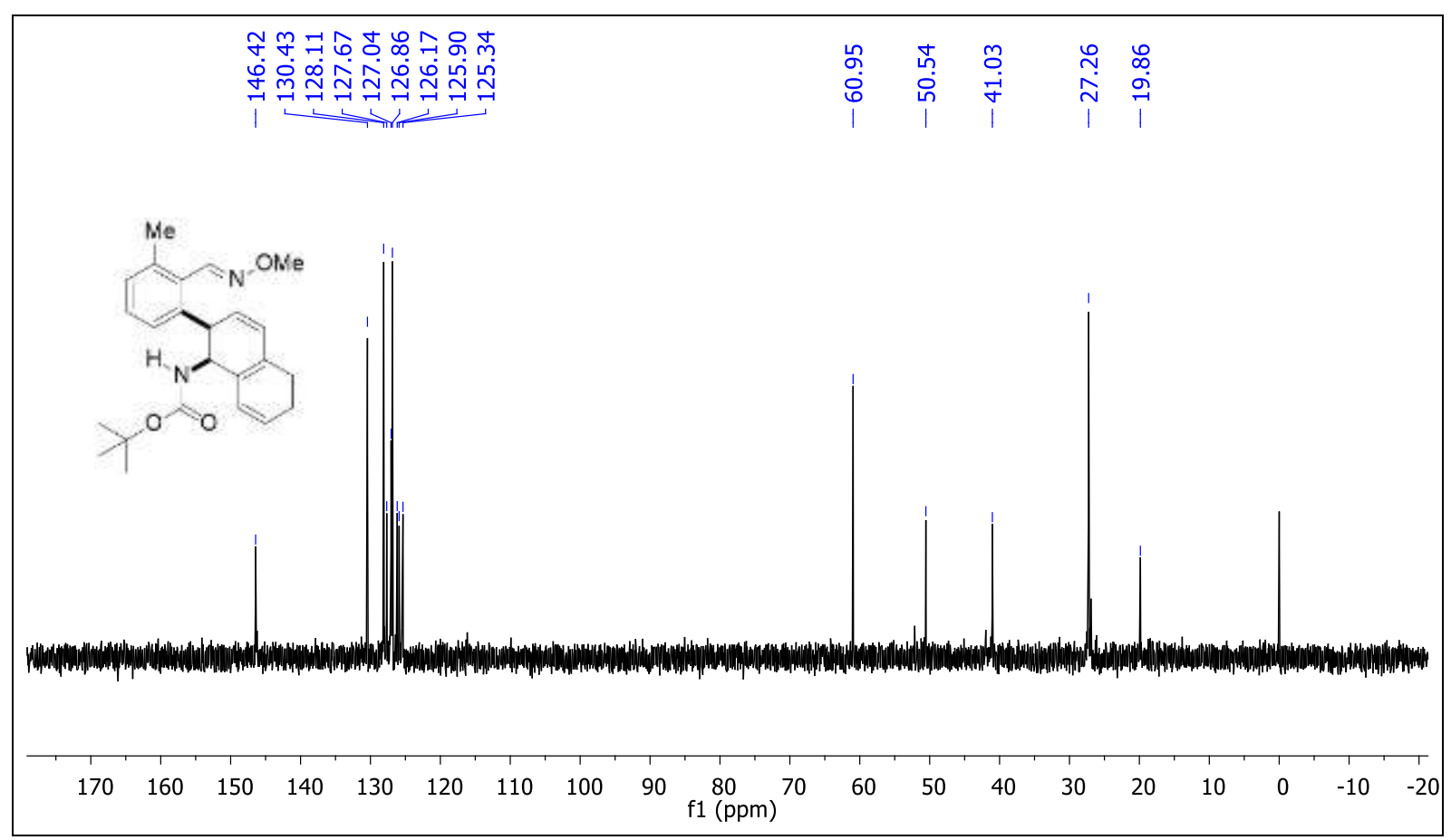


${ }^{1} \mathrm{H}$ and ${ }^{13} \mathrm{C}\left\{{ }^{1} \mathrm{H}\right\}$ NMR Spectra of Compound 3ca $\left(\mathrm{CDCl}_{3}, 400 \mathrm{MHz}\right)$.
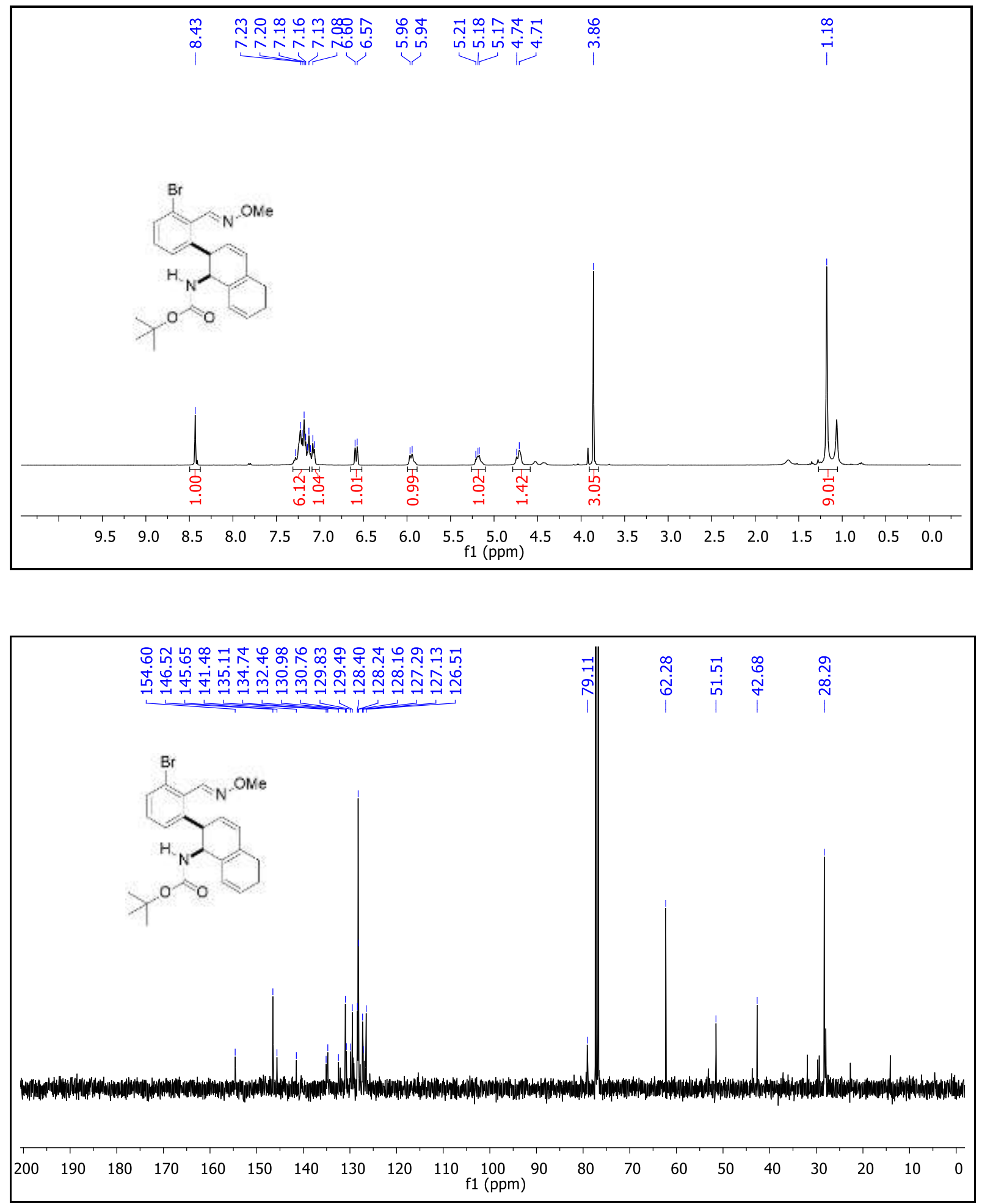
DEPT (135) NMR Spectrum of Compound 3ca $\left(\mathrm{CDCl}_{3}, 101 \mathrm{MHz}\right)$.

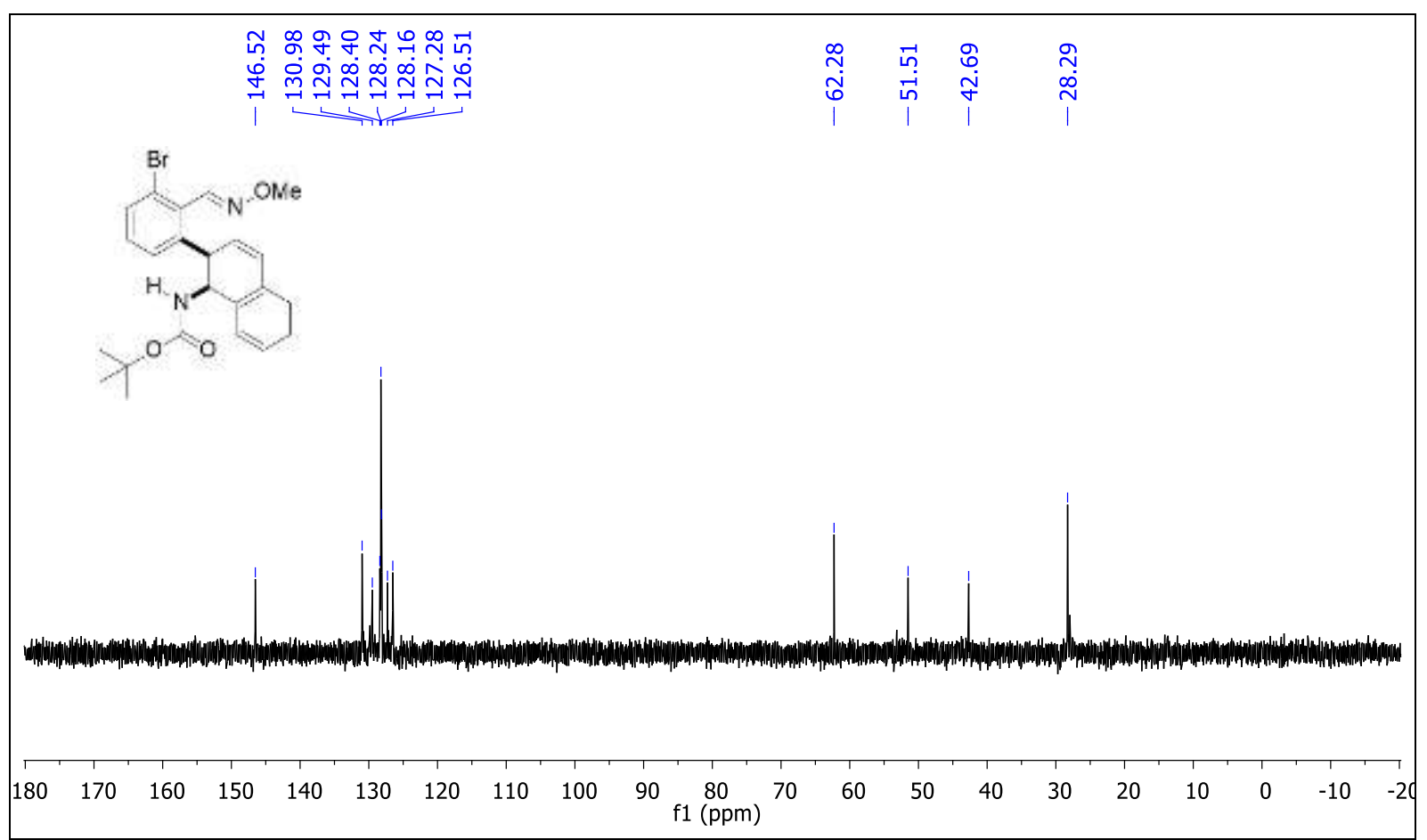


${ }^{1} \mathrm{H}$ and ${ }^{13} \mathrm{C}\left\{{ }^{1} \mathrm{H}\right\}$ NMR Spectra of Compound 3da $\left(\mathrm{CDCl}_{3}, 400 \mathrm{MHz}\right)$.
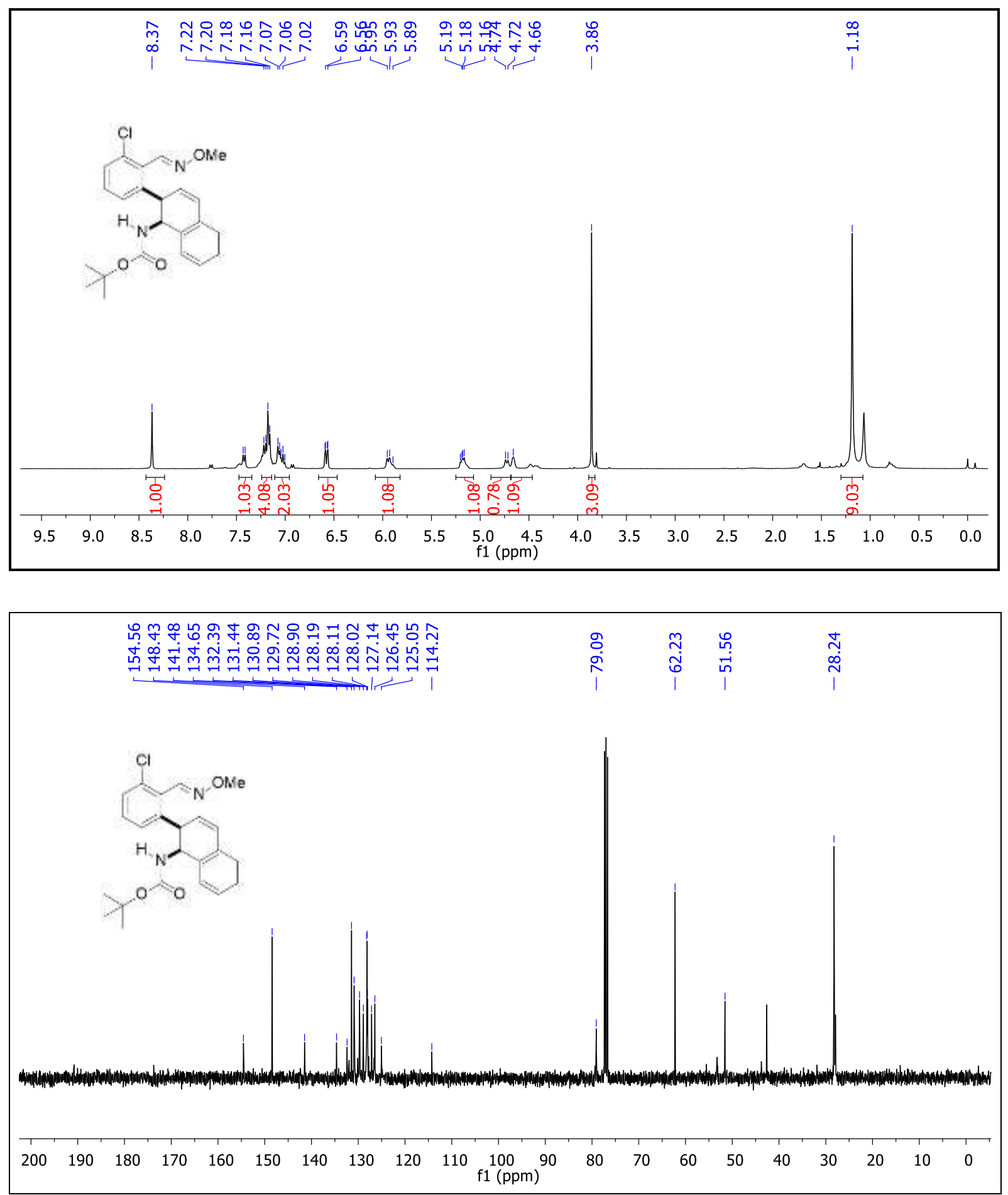
DEPT (135) NMR Spectrum of Compound 3da $\left(\mathrm{CDCl}_{3}, 101 \mathrm{MHz}\right)$.

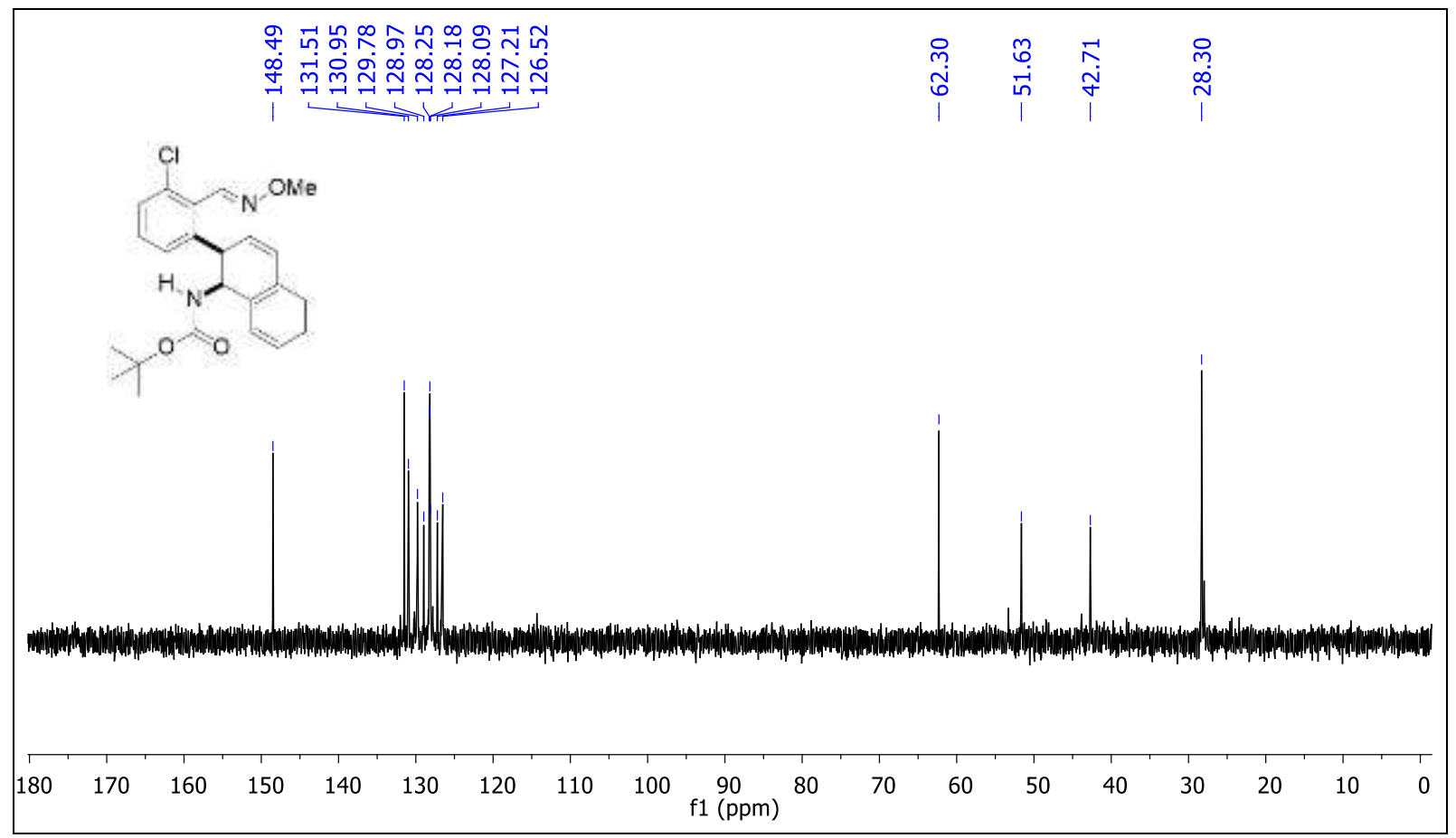


${ }^{1} \mathrm{H}$ and ${ }^{13} \mathrm{C}\left\{{ }^{1} \mathrm{H}\right\}$ NMR Spectra of Compound 3ea $\left(\mathrm{CDCl}_{3}, 400 \mathrm{MHz}\right)$.
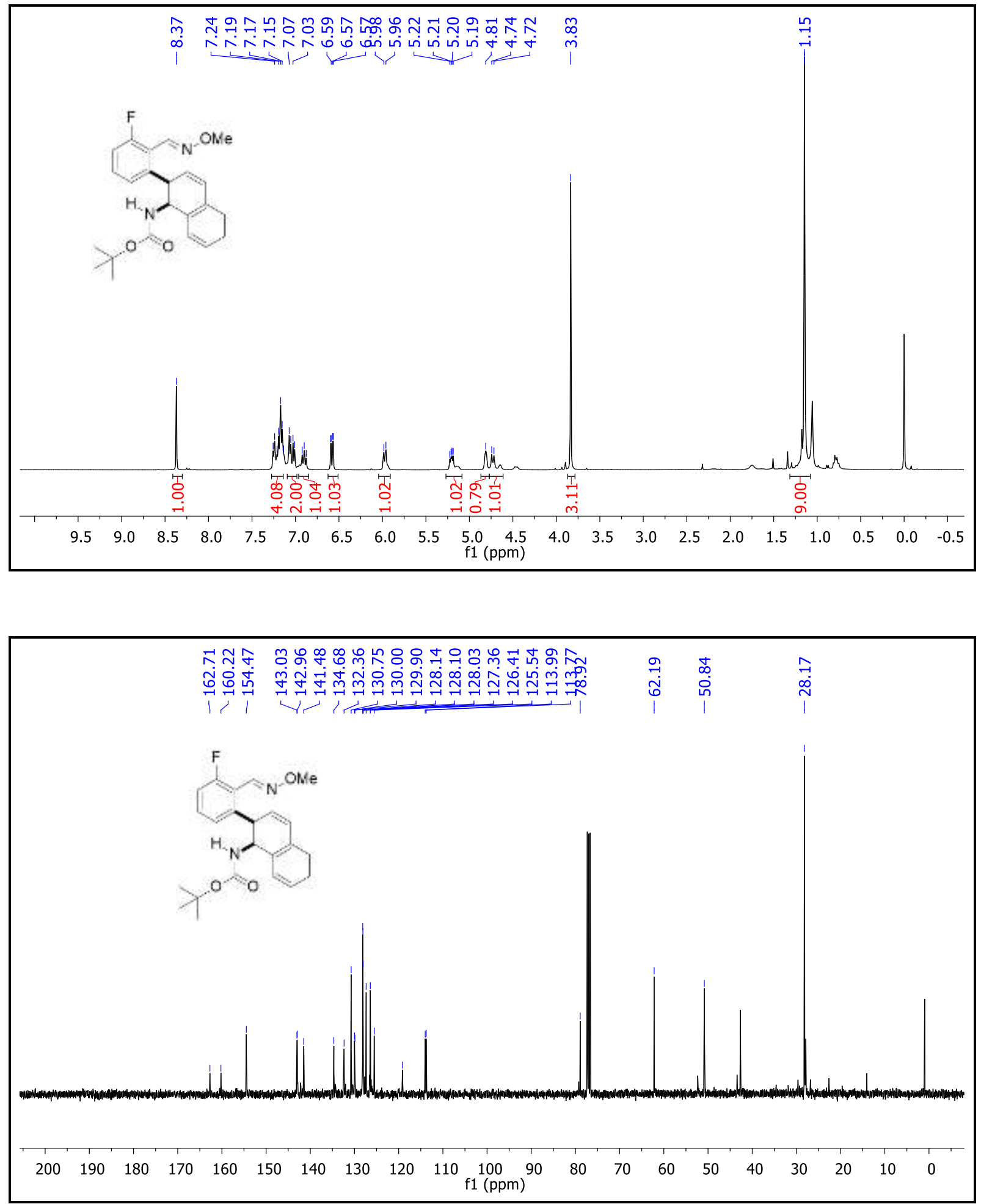
DEPT (135) NMR Spectrum of Compound 3ea $\left(\mathrm{CDCl}_{3}, 101 \mathrm{MHz}\right)$.

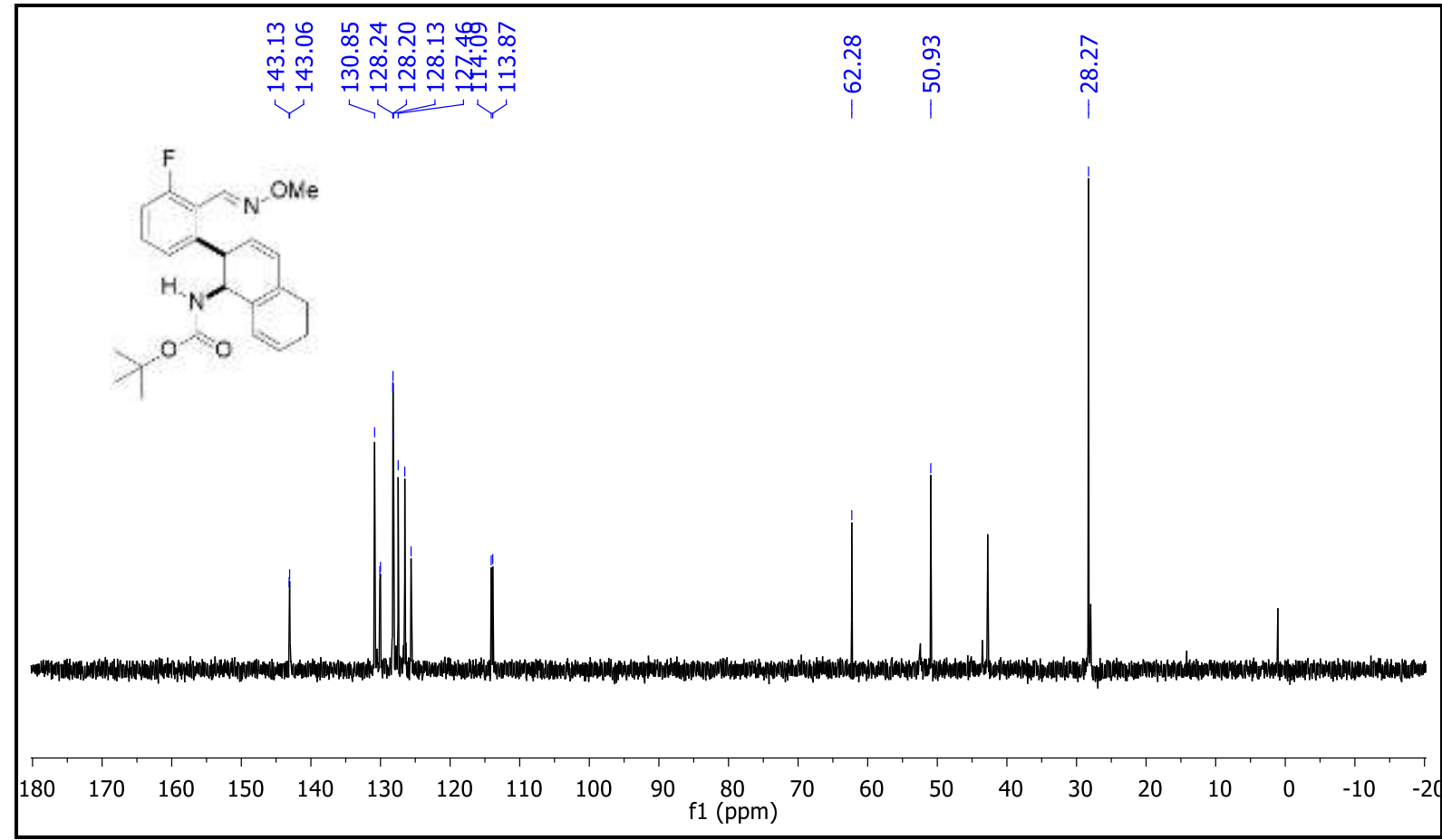

${ }^{19} \mathrm{~F}$ NMR Spectrum of Compound 3ea $\left(\mathrm{CDCl}_{3}, 471 \mathrm{MHz}\right)$.

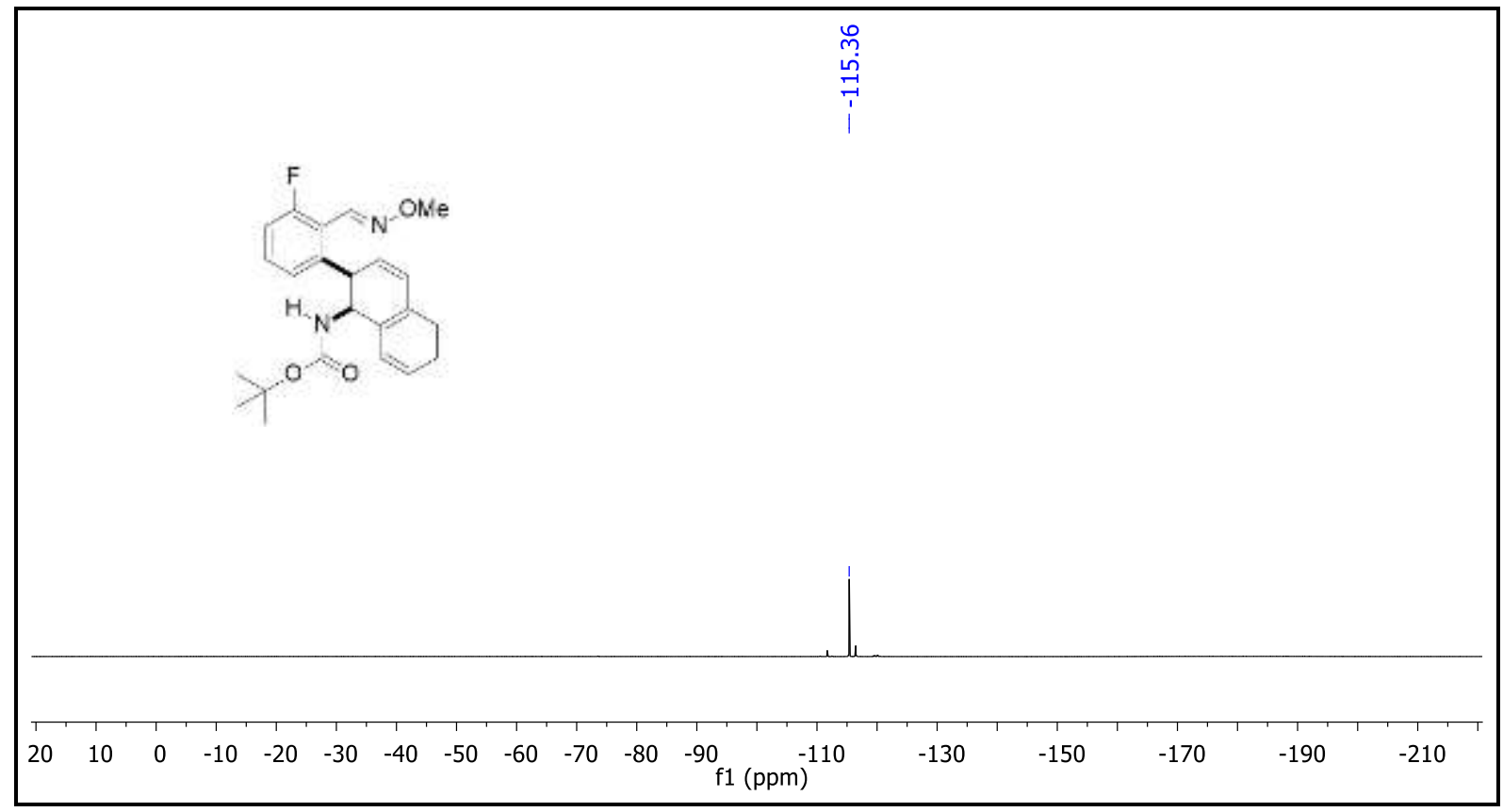


${ }^{1} \mathrm{H}$ and ${ }^{13} \mathrm{C}\left\{{ }^{1} \mathrm{H}\right\}$ NMR Spectra of Compound $3 f a\left(\mathrm{CDCl}_{3}, 400 \mathrm{MHz}\right)$.
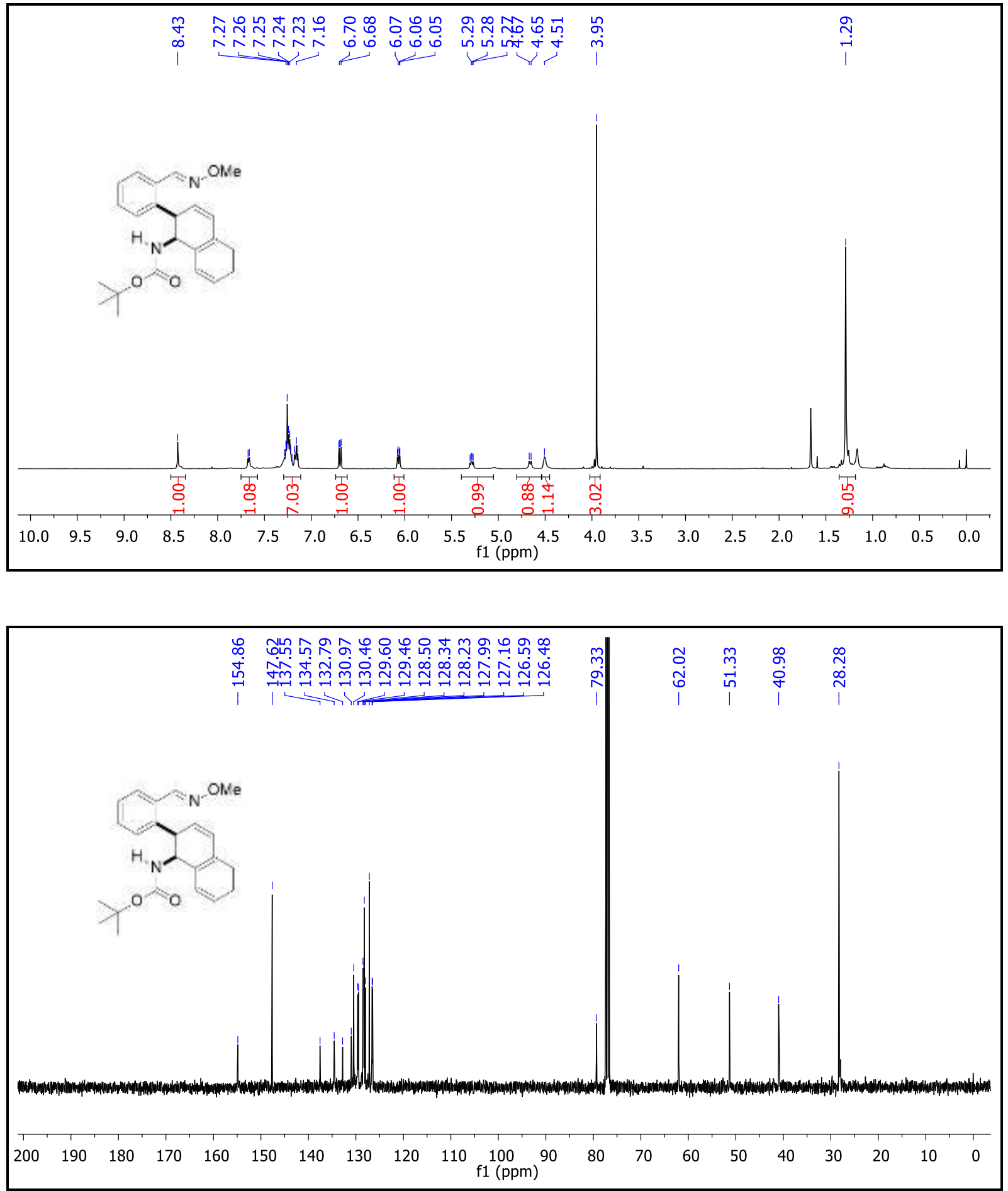
DEPT (135) NMR Spectrum of Compound $\mathbf{3 f a}\left(\mathrm{CDCl}_{3}, 101 \mathrm{MHz}\right)$.

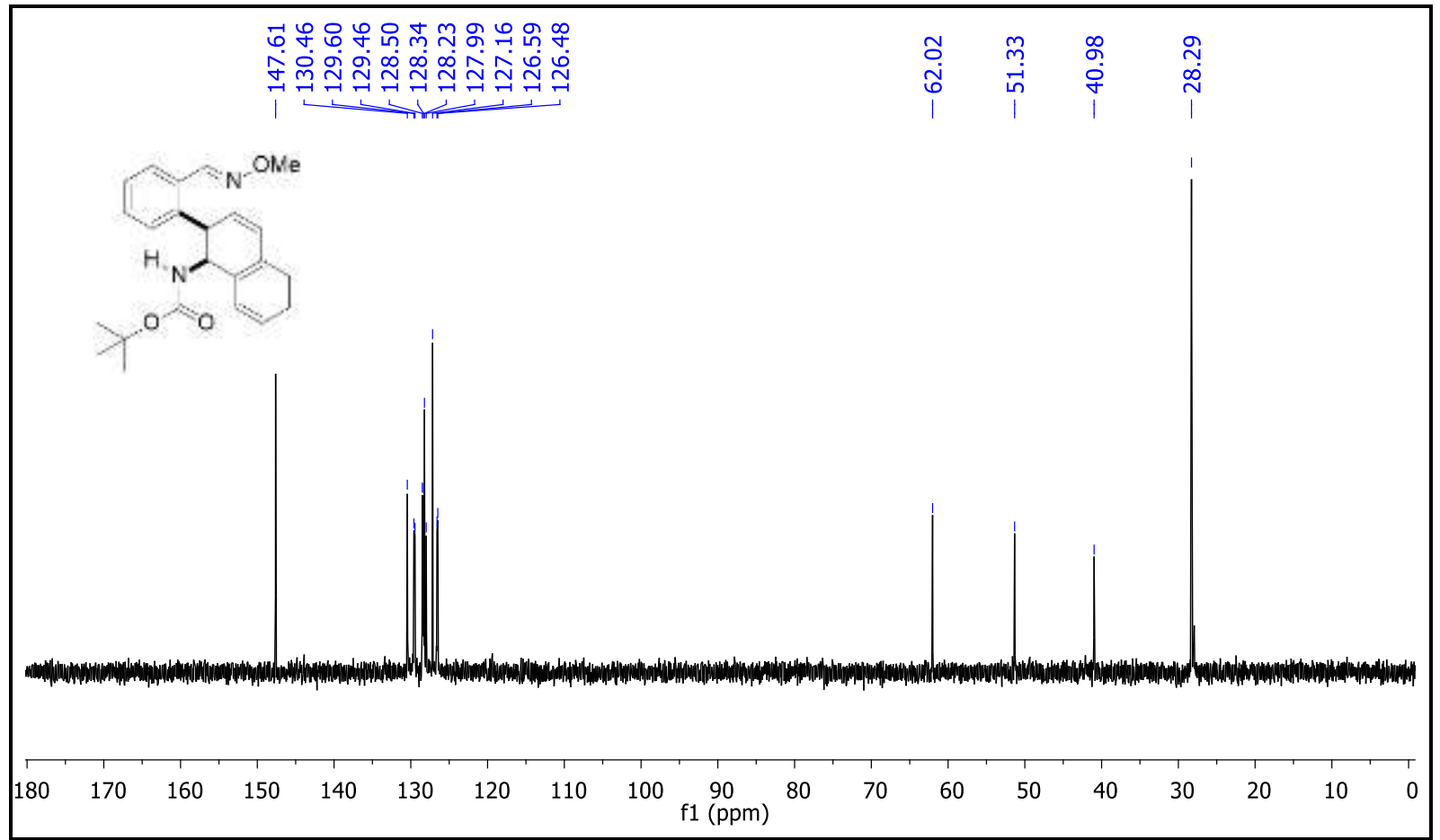


${ }^{1} \mathrm{H}$ and ${ }^{13} \mathrm{C}\left\{{ }^{1} \mathrm{H}\right\}$ NMR Spectra of Compound 3ga $\left(\mathrm{CDCl}_{3}, 400 \mathrm{MHz}\right)$.
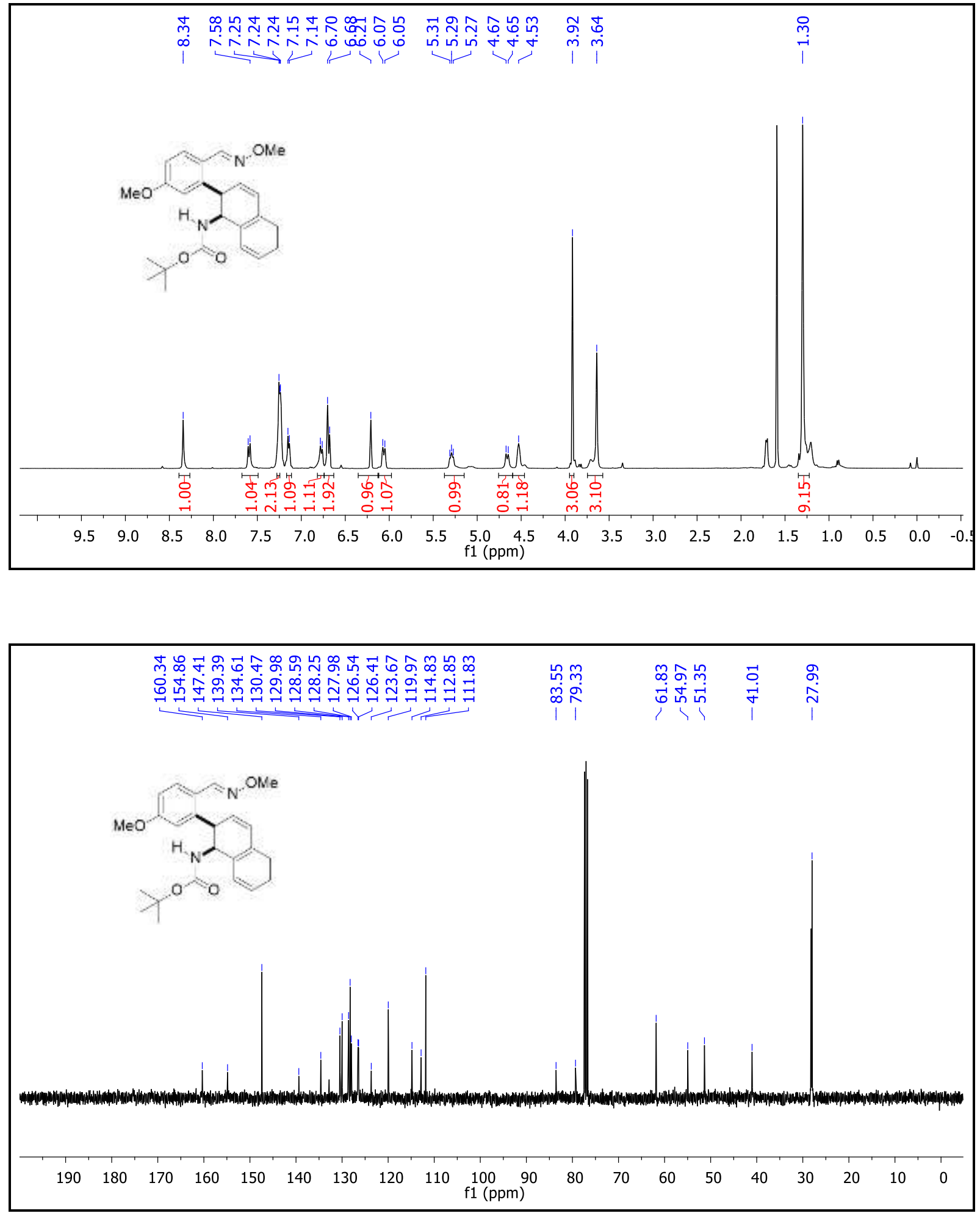
DEPT (135) NMR Spectrum of Compound 3ga $\left(\mathrm{CDCl}_{3}, 101 \mathrm{MHz}\right)$.

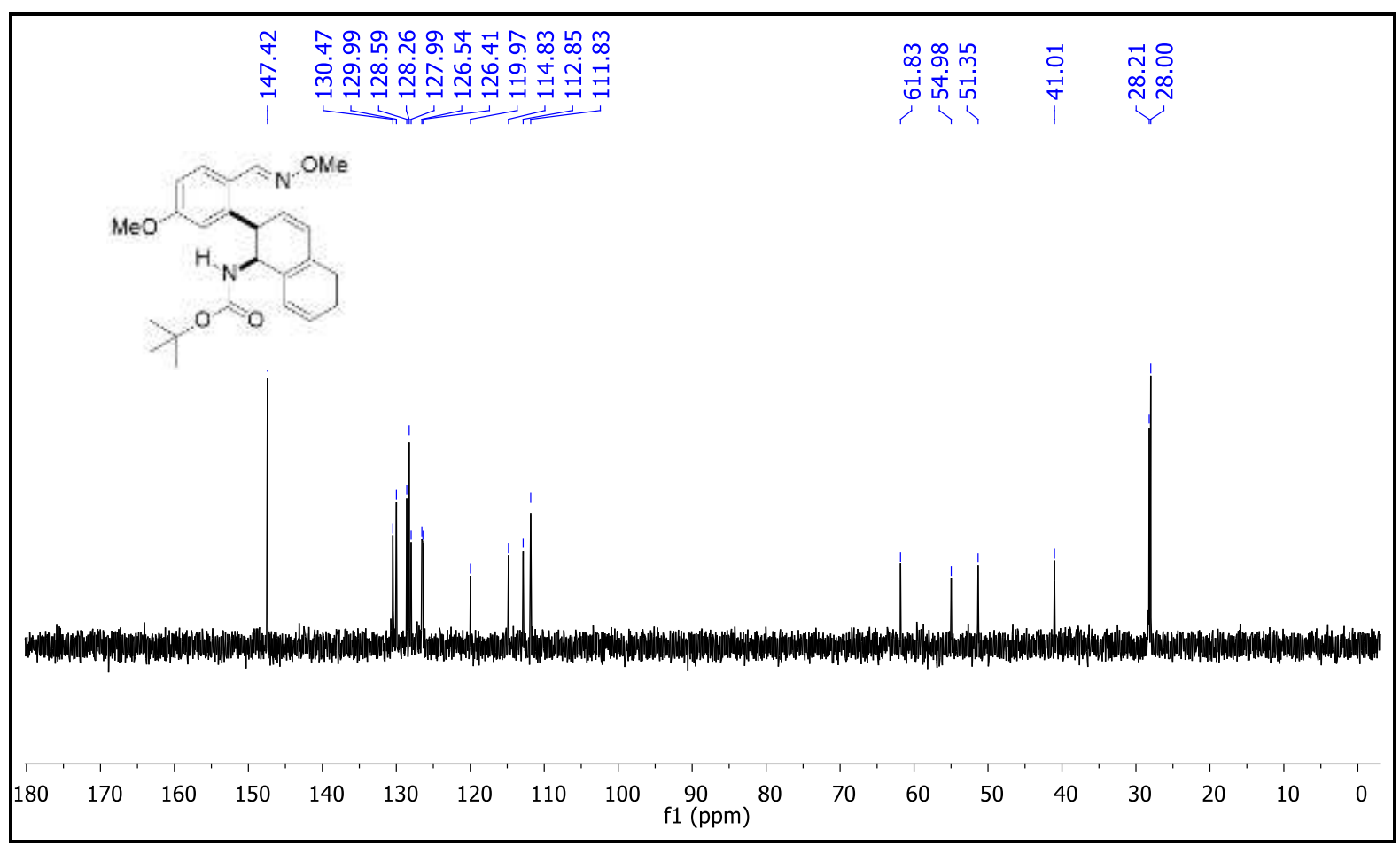


${ }^{1} \mathrm{H}$ and ${ }^{13} \mathrm{C}\left\{{ }^{1} \mathrm{H}\right\}$ NMR Spectra of Compound 3ha $\left(\mathrm{CDCl}_{3}, 400 \mathrm{MHz}\right)$.
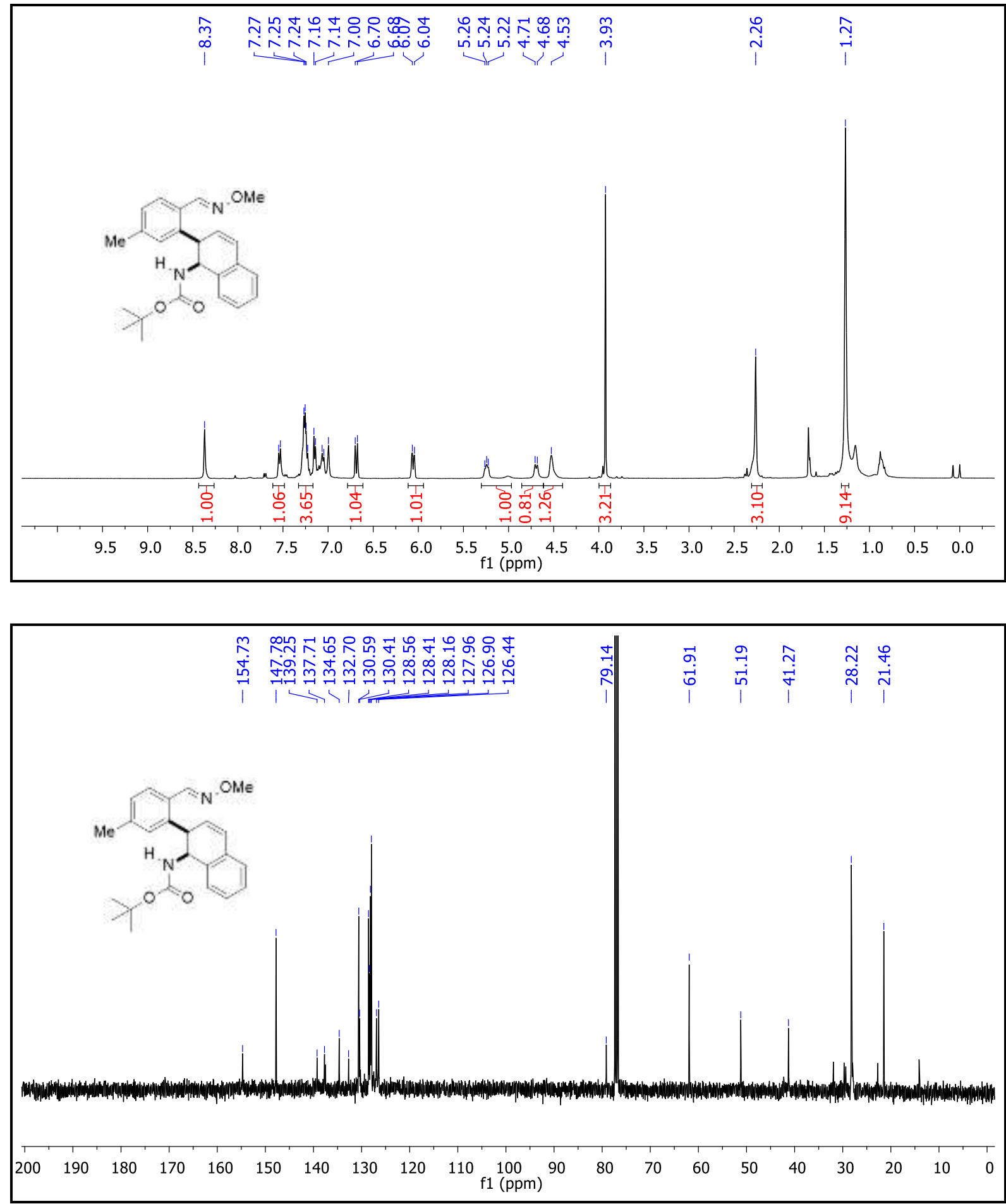
DEPT (135) NMR Spectrum of Compound 3ha $\left(\mathrm{CDCl}_{3}, 101 \mathrm{MHz}\right)$.

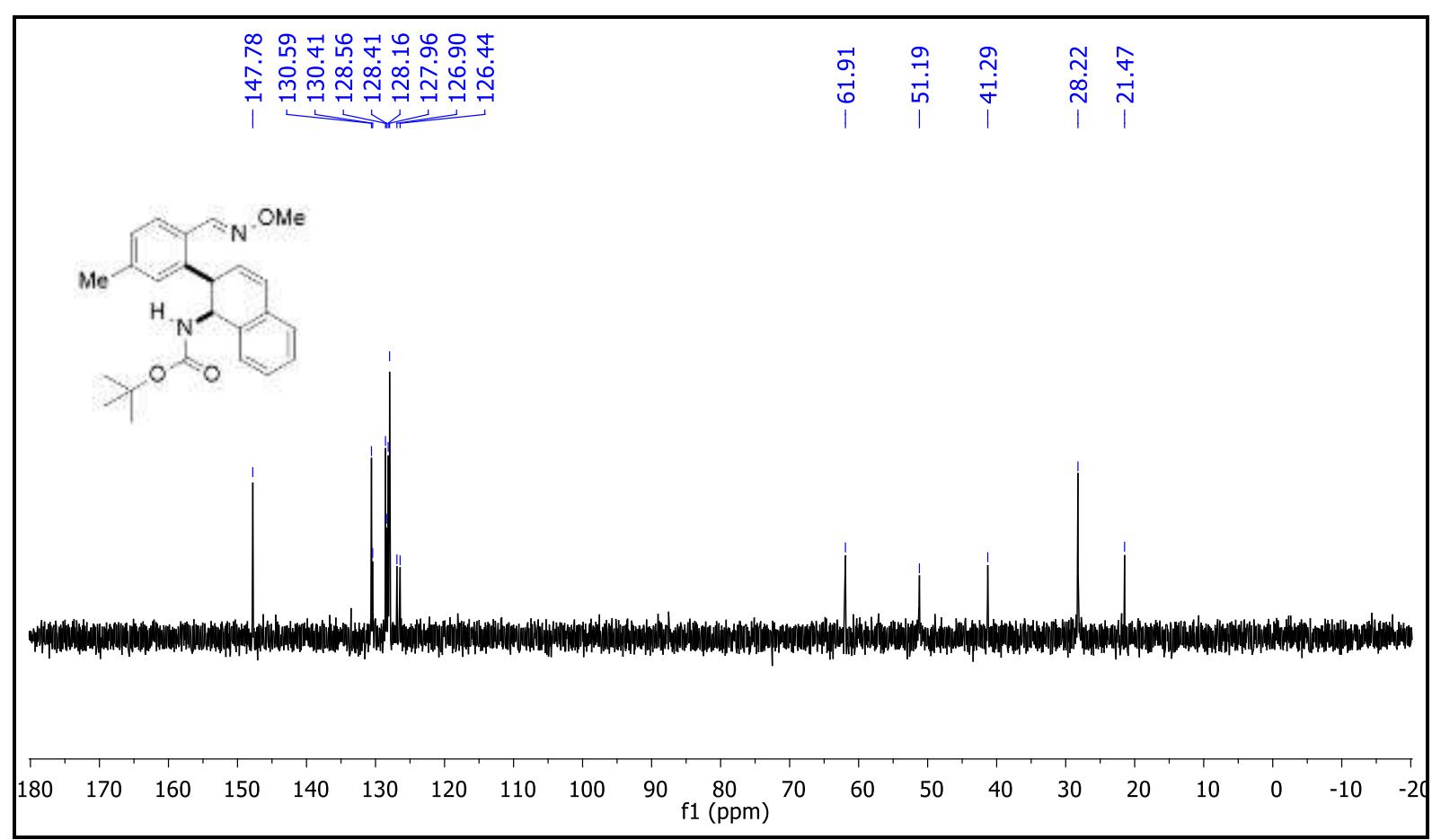


${ }^{1} \mathrm{H}$ and ${ }^{13} \mathrm{C}\left\{{ }^{1} \mathrm{H}\right\}$ NMR Spectra of Compound 3ia $\left(\mathrm{CDCl}_{3}, 400 \mathrm{MHz}\right)$.
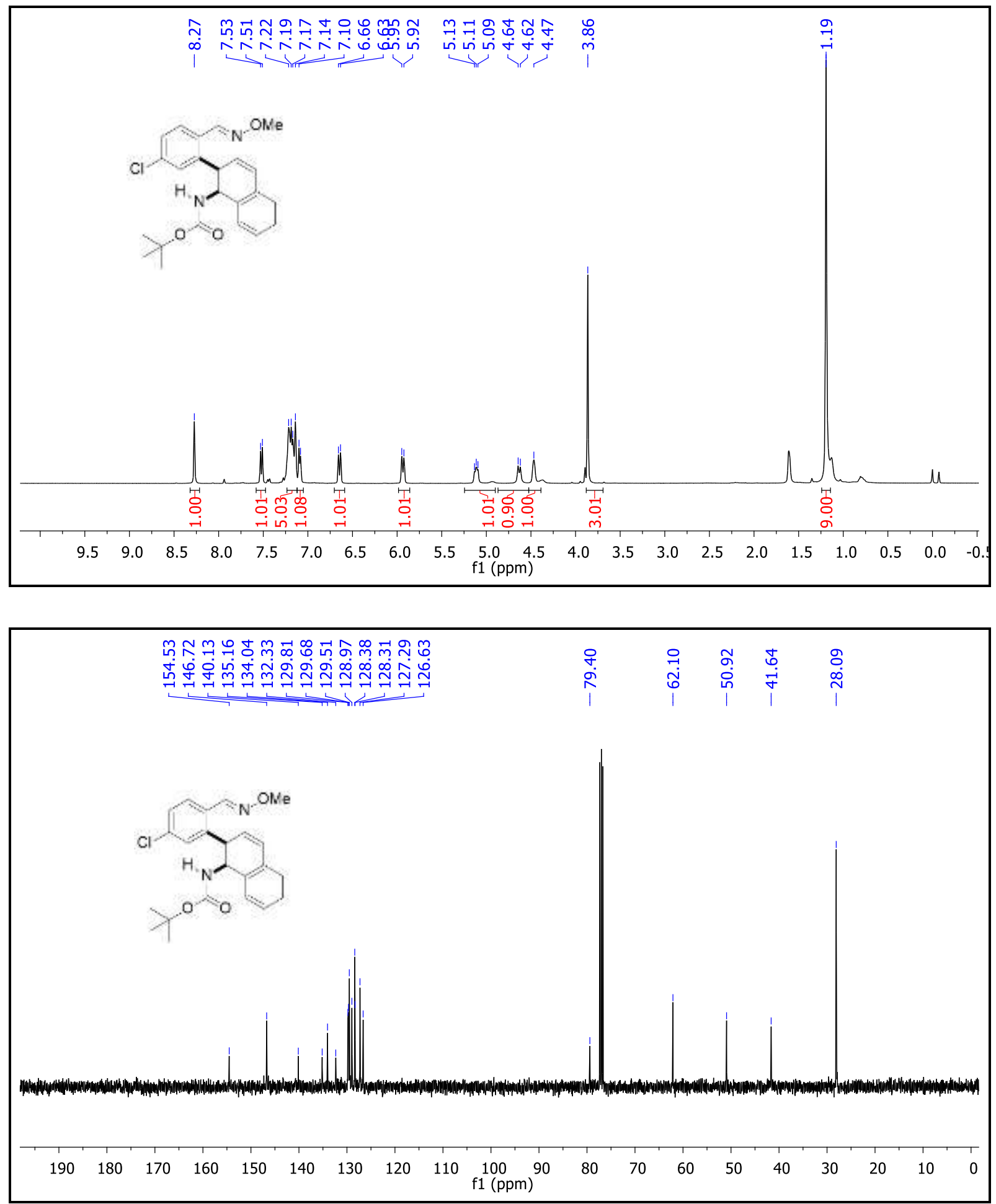
DEPT (135) NMR Spectrum of Compound 3ia $\left(\mathrm{CDCl}_{3}, 101 \mathrm{MHz}\right)$.

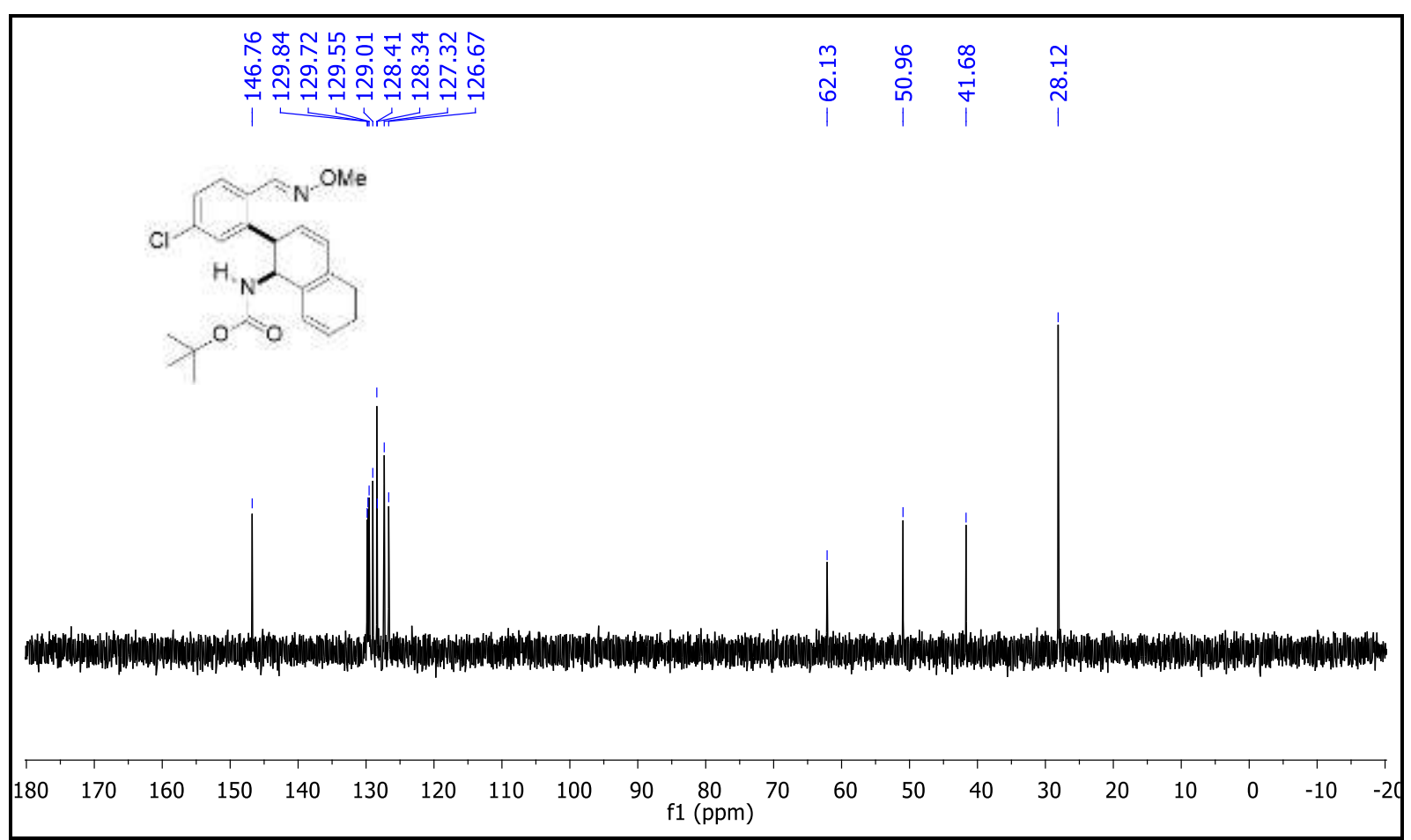


${ }^{1} \mathrm{H}$ and ${ }^{13} \mathrm{C}\left\{{ }^{1} \mathrm{H}\right\}$ NMR Spectra of Compound 3ja $\left(\mathrm{CDCl}_{3}, 400 \mathrm{MHz}\right)$.
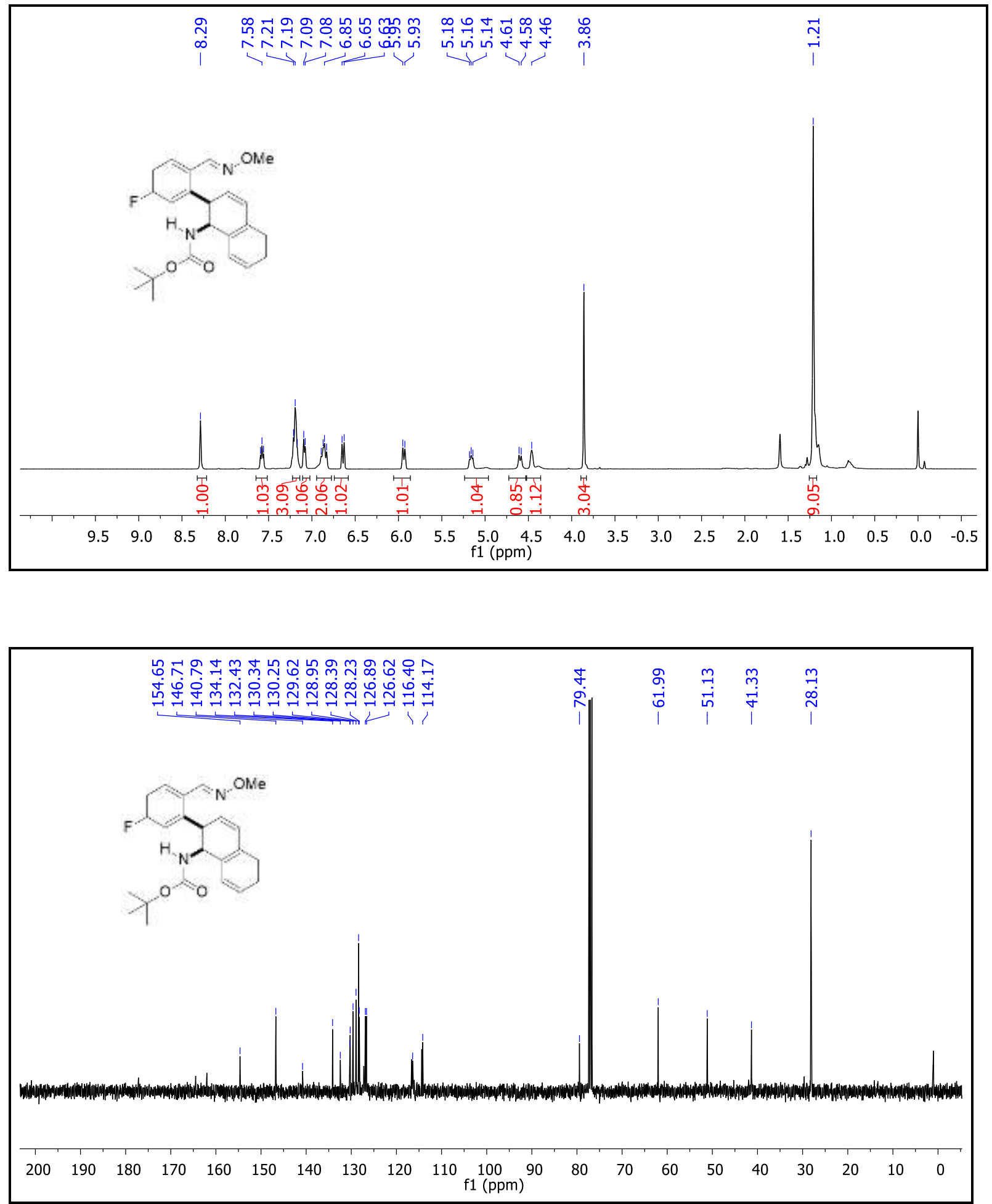
DEPT (135) NMR Spectrum of Compound 3ja $\left(\mathrm{CDCl}_{3}, 101 \mathrm{MHz}\right)$.

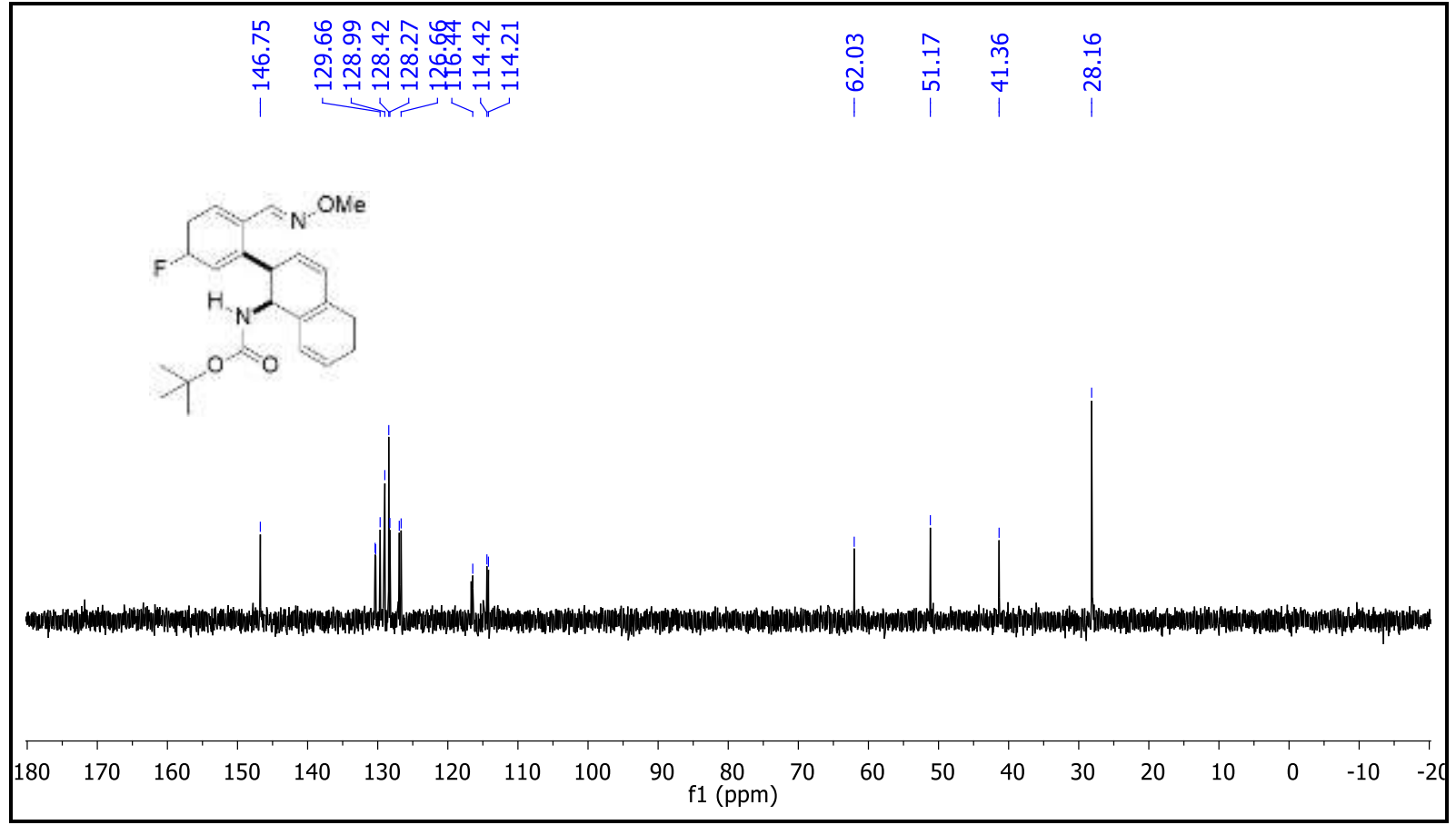

${ }^{19} \mathrm{~F}$ NMR Spectrum of Compound $\mathbf{3 j a}\left(\mathrm{CDCl}_{3}, 471 \mathrm{MHz}\right)$.

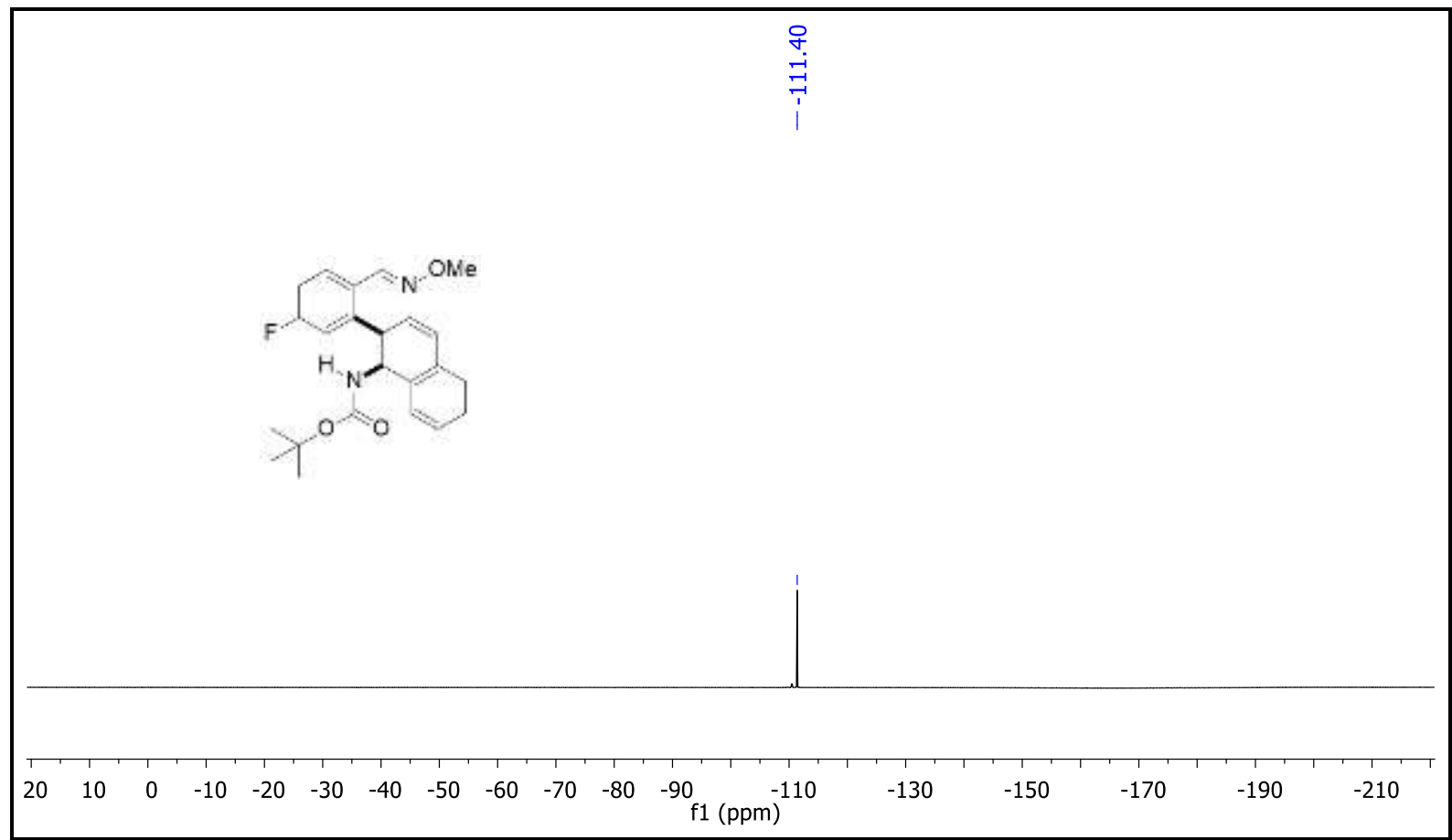


${ }^{1} \mathrm{H}$ and ${ }^{13} \mathrm{C}\left\{{ }^{1} \mathrm{H}\right\}$ NMR Spectra of Compound 3ka $\left(\mathrm{CDCl}_{3}, 400 \mathrm{MHz}\right)$.
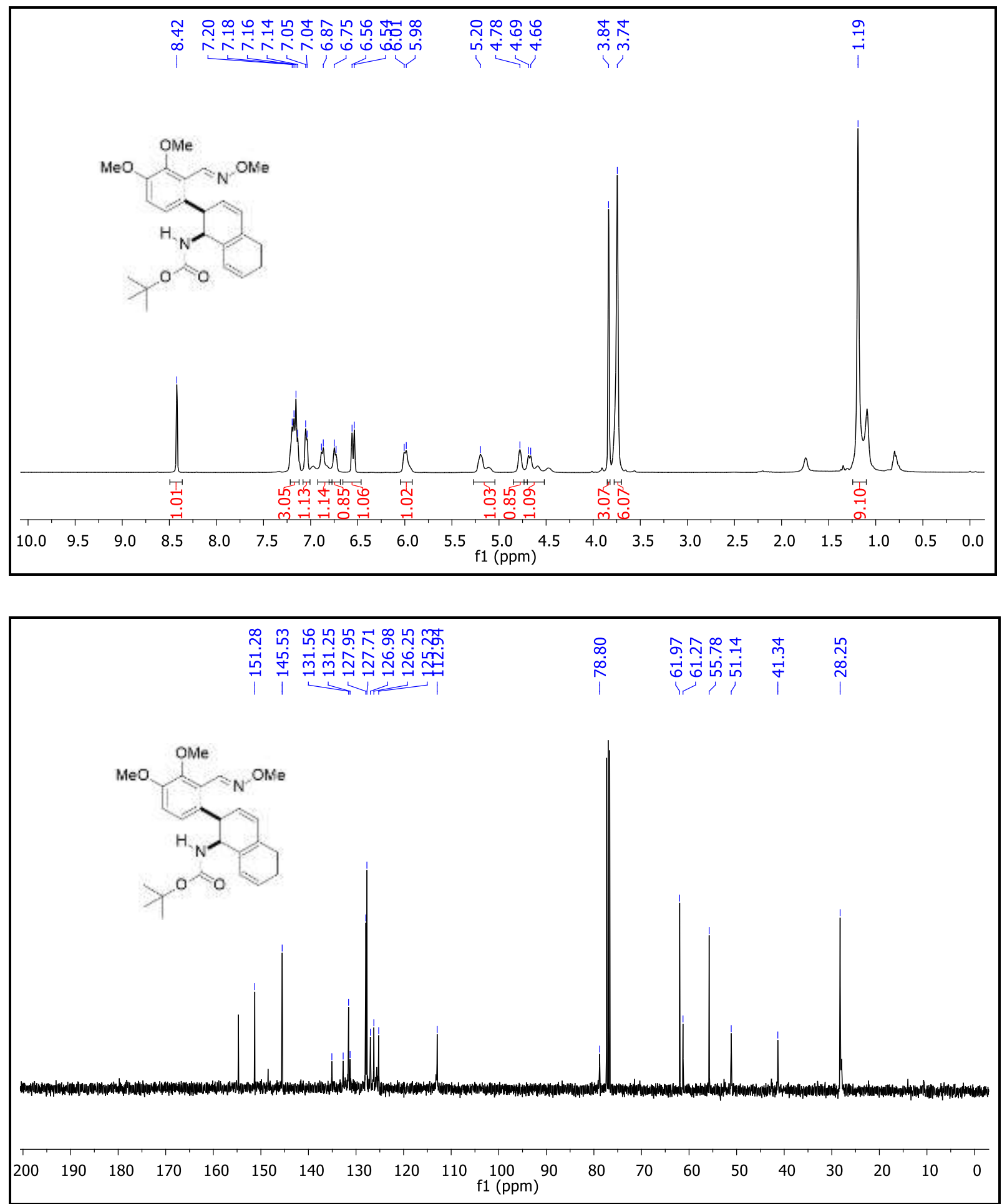
DEPT (135) NMR Spectrum of Compound 3ka $\left(\mathrm{CDCl}_{3}, 101 \mathrm{MHz}\right)$.

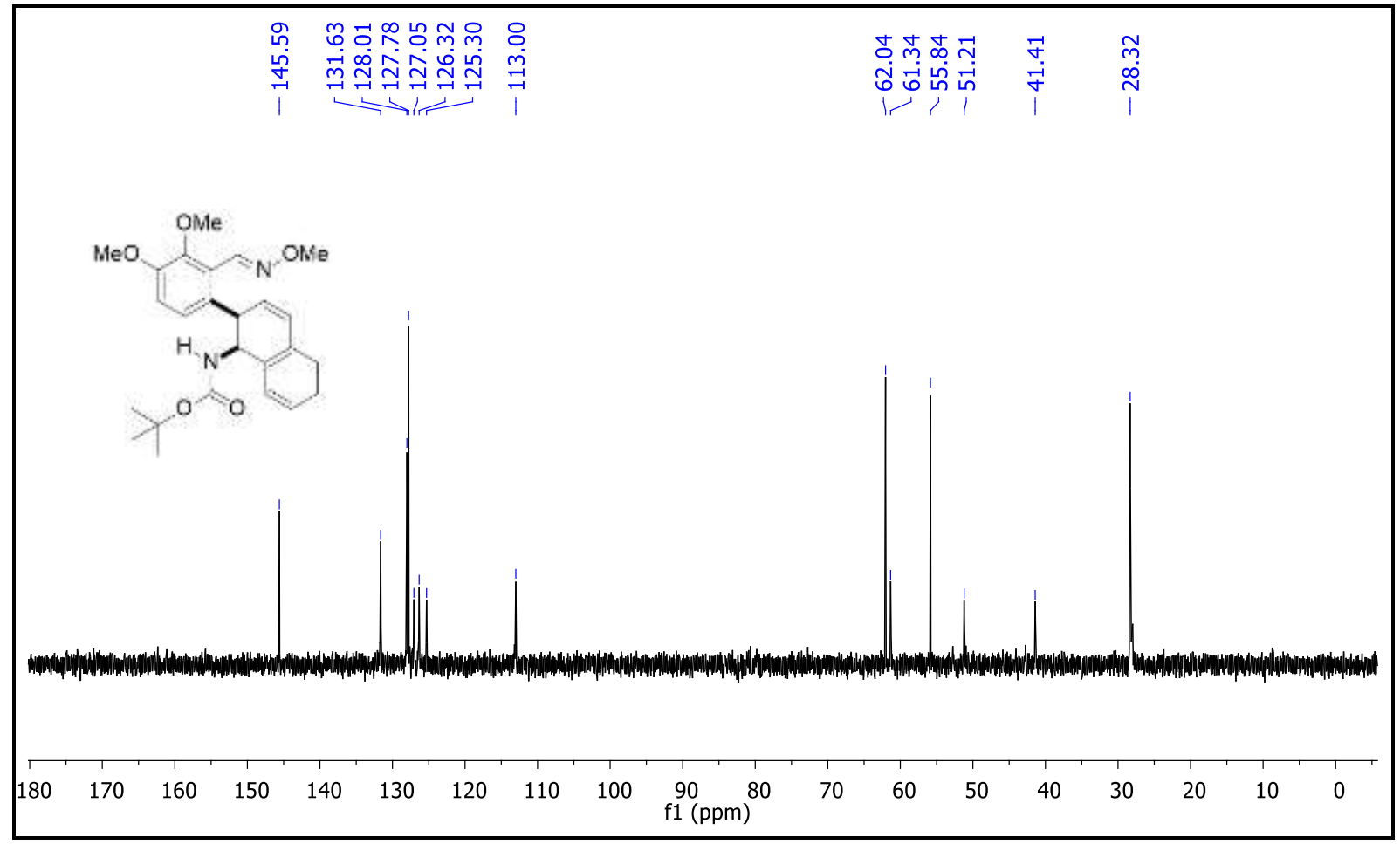


${ }^{1} \mathrm{H}$ and ${ }^{13} \mathrm{C}\left\{{ }^{1} \mathrm{H}\right\}$ NMR Spectra of Compound 3la $\left(\mathrm{CDCl}_{3}, 500 \mathrm{MHz}\right)$.
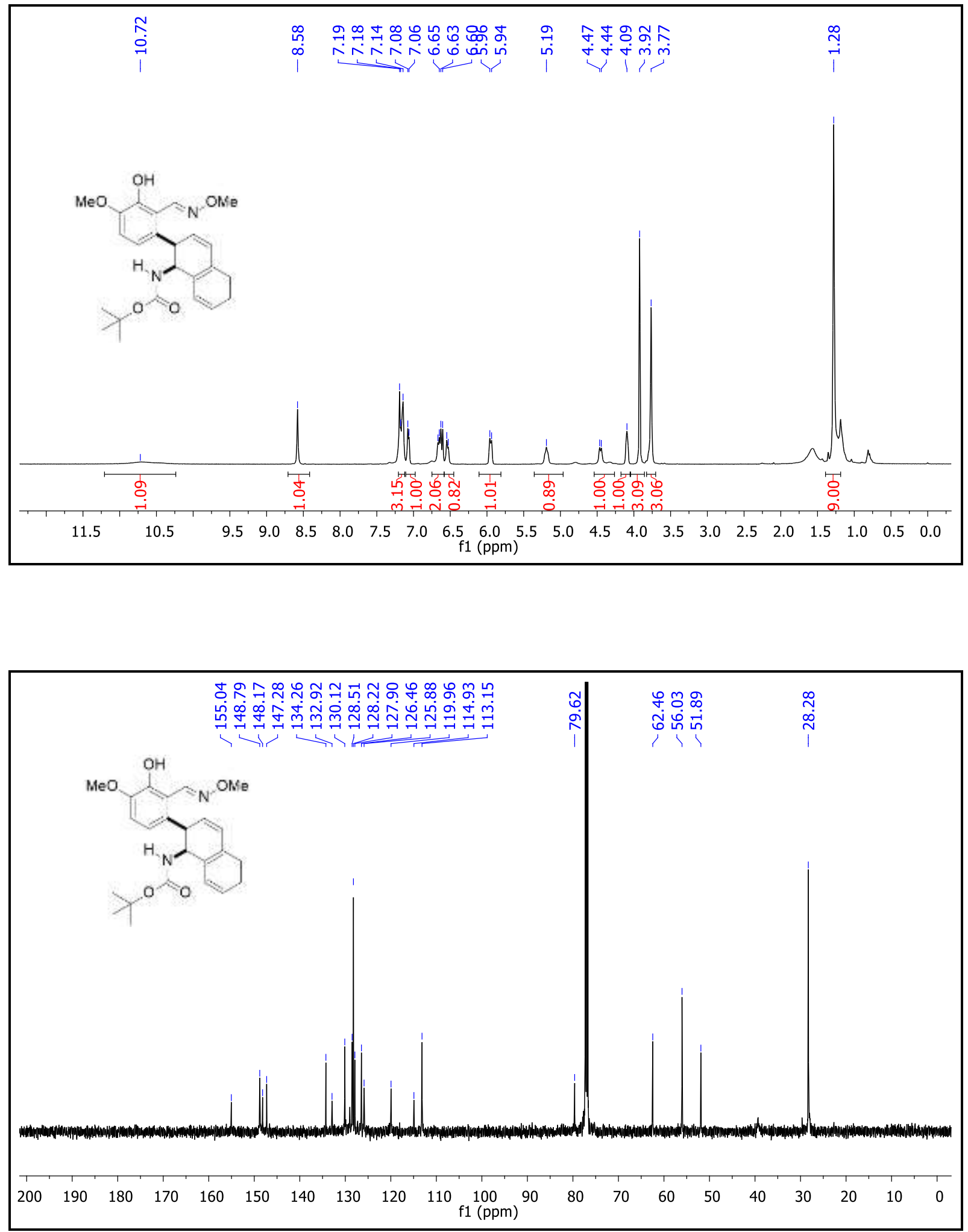
DEPT (135) NMR Spectrum of Compound 3la $\left(\mathrm{CDCl}_{3}, 126 \mathrm{MHz}\right)$.

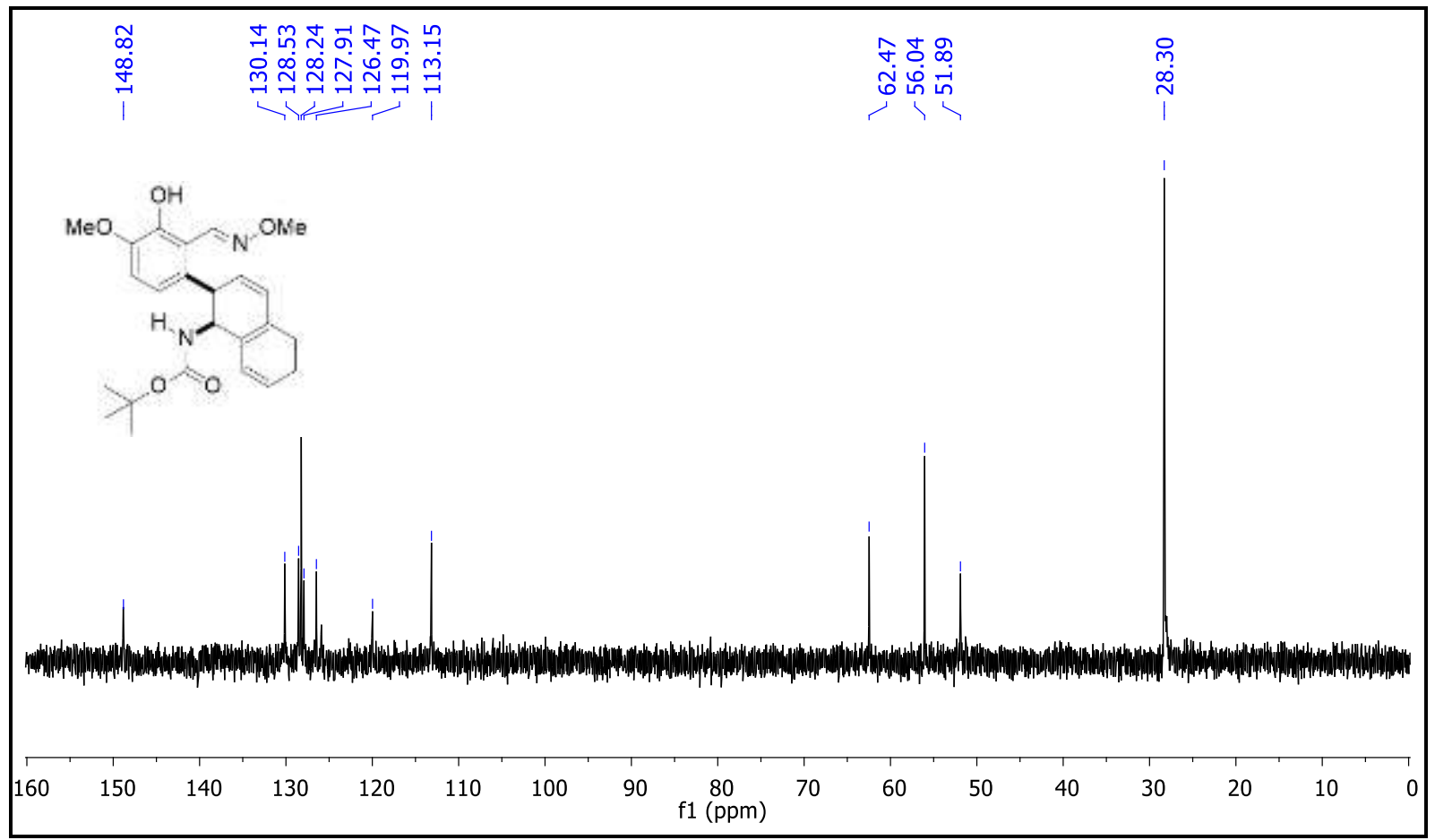


${ }^{1} \mathrm{H}$ and ${ }^{13} \mathrm{C}\left\{{ }^{1} \mathrm{H}\right\}$ NMR Spectra of Compound 3ma $\left(\mathrm{CDCl}_{3}, 400 \mathrm{MHz}\right)$.
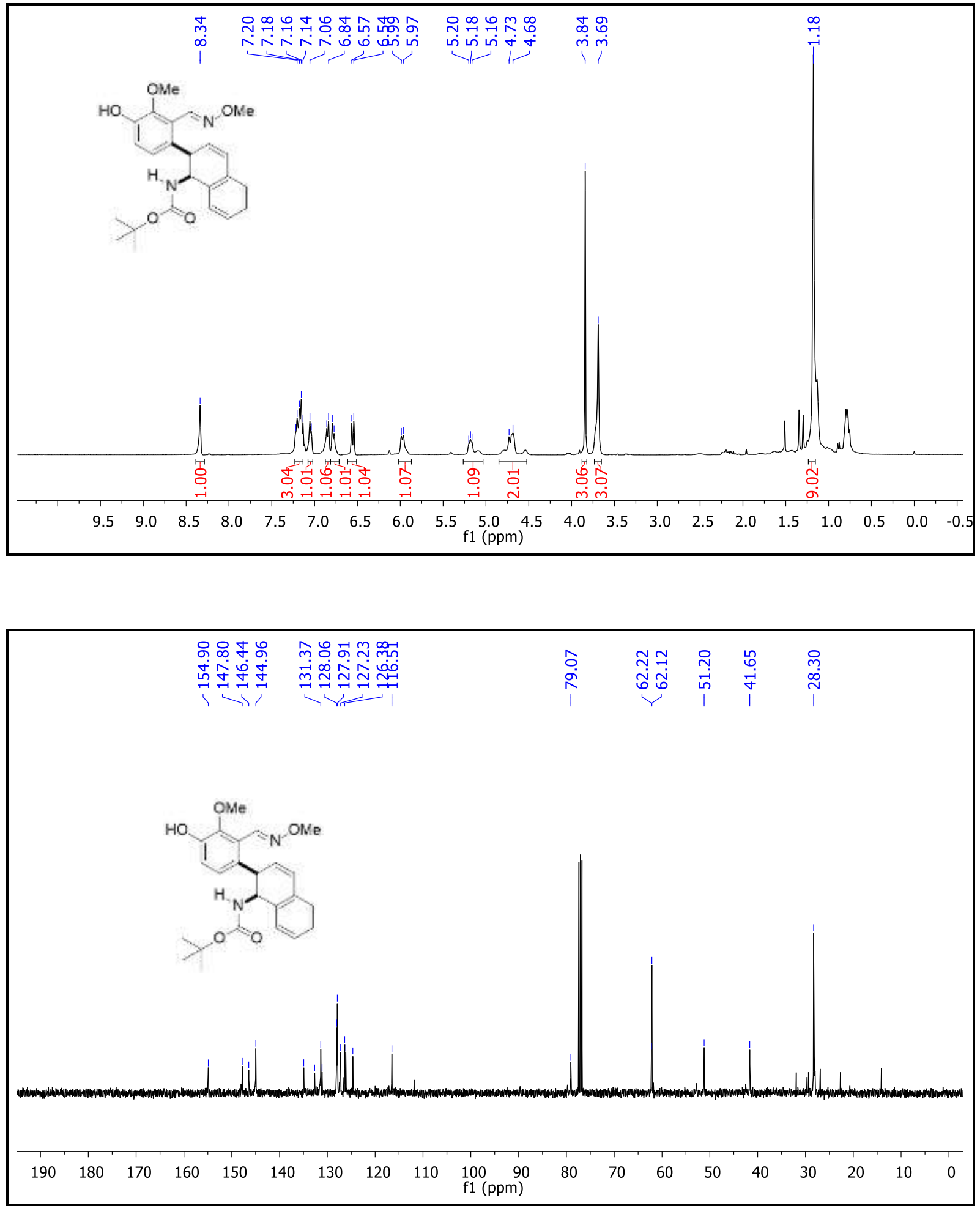
DEPT (135) NMR Spectrum of Compound 3ma $\left(\mathrm{CDCl}_{3}, 101 \mathrm{MHz}\right)$.

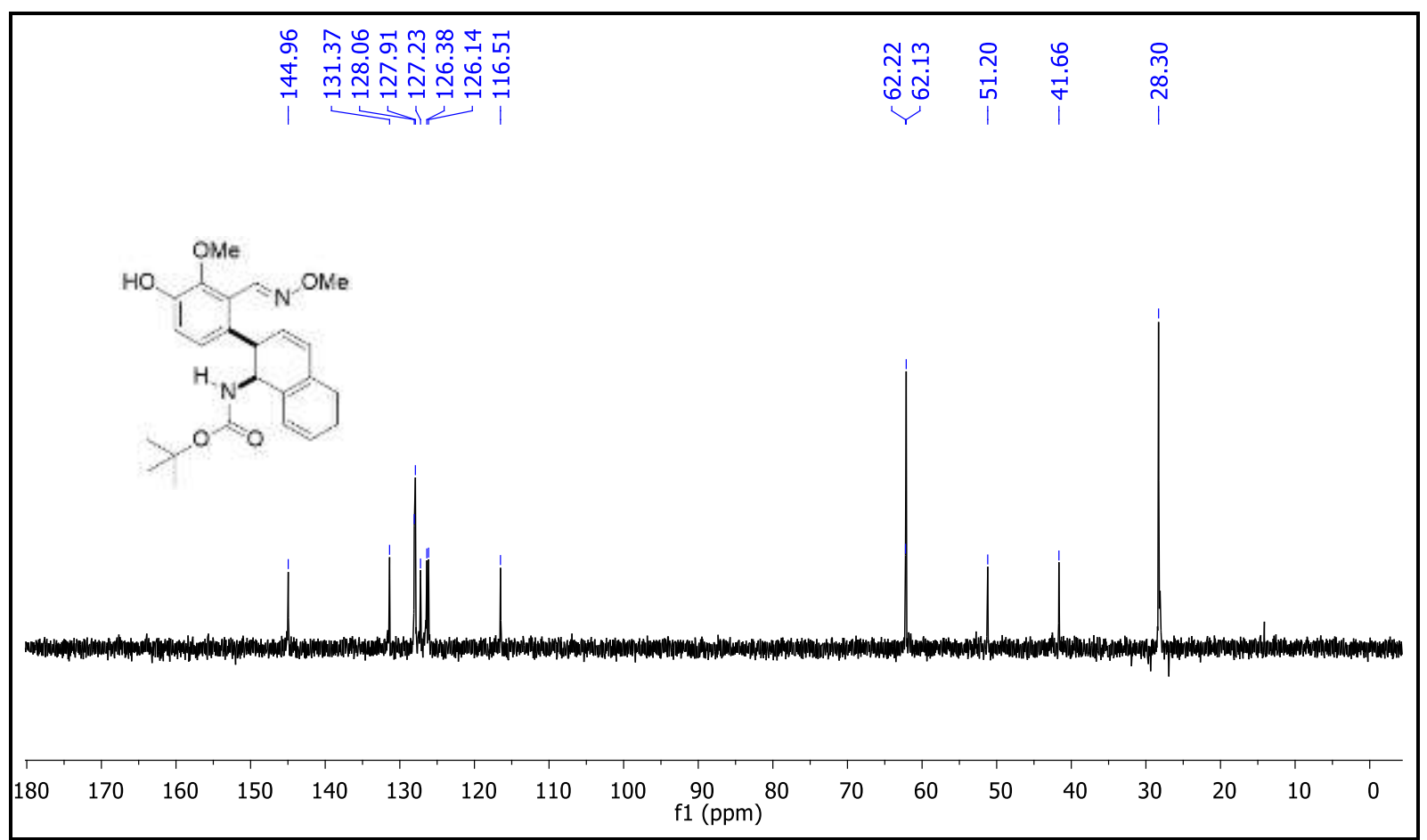


${ }^{1} \mathrm{H}$ and ${ }^{13} \mathrm{C}\left\{{ }^{1} \mathrm{H}\right\}$ NMR Spectra of Compound 3na $\left(\mathrm{CDCl}_{3}, 400 \mathrm{MHz}\right)$.
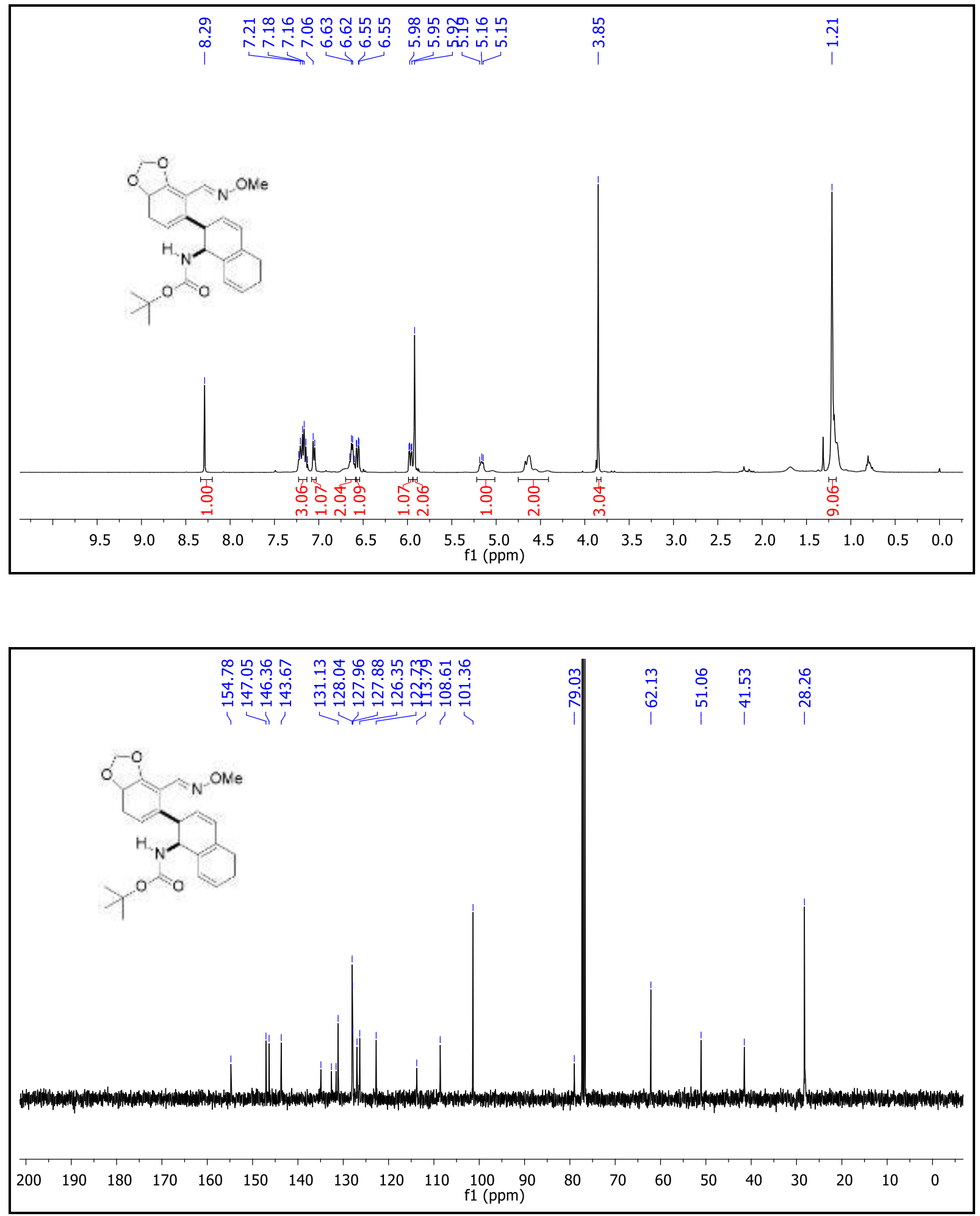
DEPT (135) NMR Spectrum of Compound 3na $\left(\mathrm{CDCl}_{3}, 101 \mathrm{MHz}\right)$.

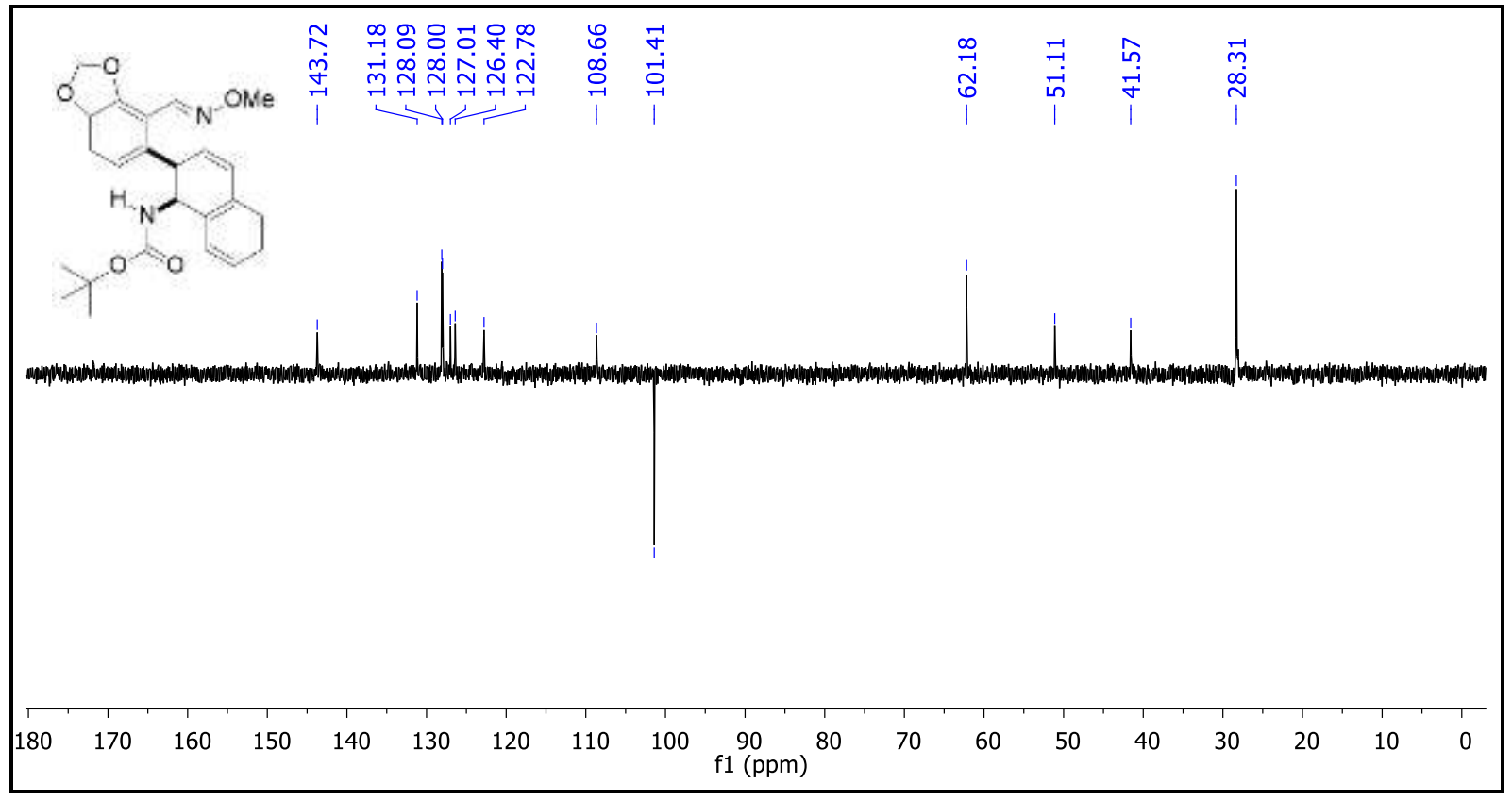


${ }^{1} \mathrm{H}$ and ${ }^{13} \mathrm{C}\left\{{ }^{1} \mathrm{H}\right\}$ NMR Spectra of Compound 3oa $\left(\mathrm{CDCl}_{3}, 400 \mathrm{MHz}\right)$.
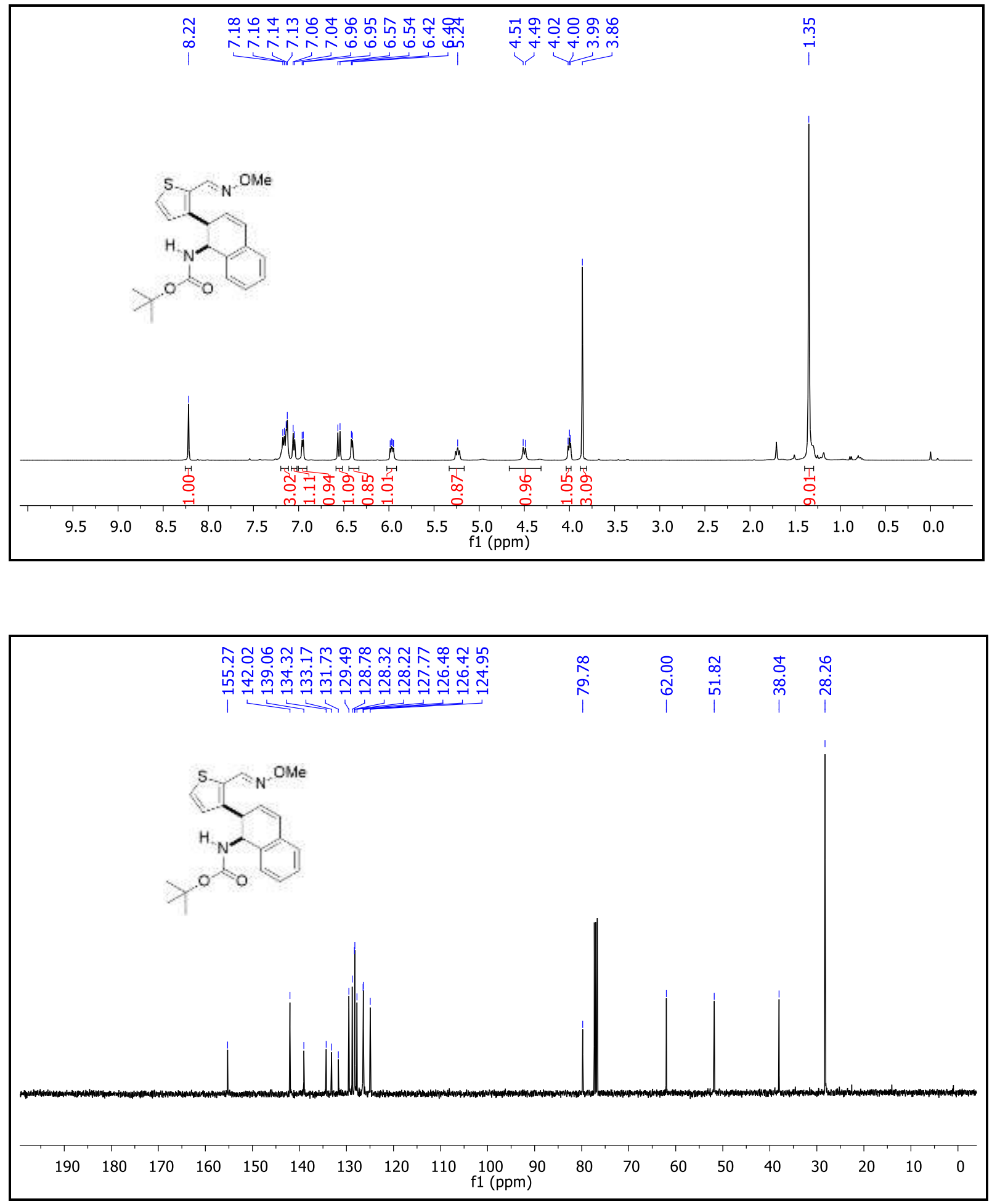
DEPT (135) NMR Spectrum of Compound 3oa $\left(\mathrm{CDCl}_{3}, 101 \mathrm{MHz}\right)$.

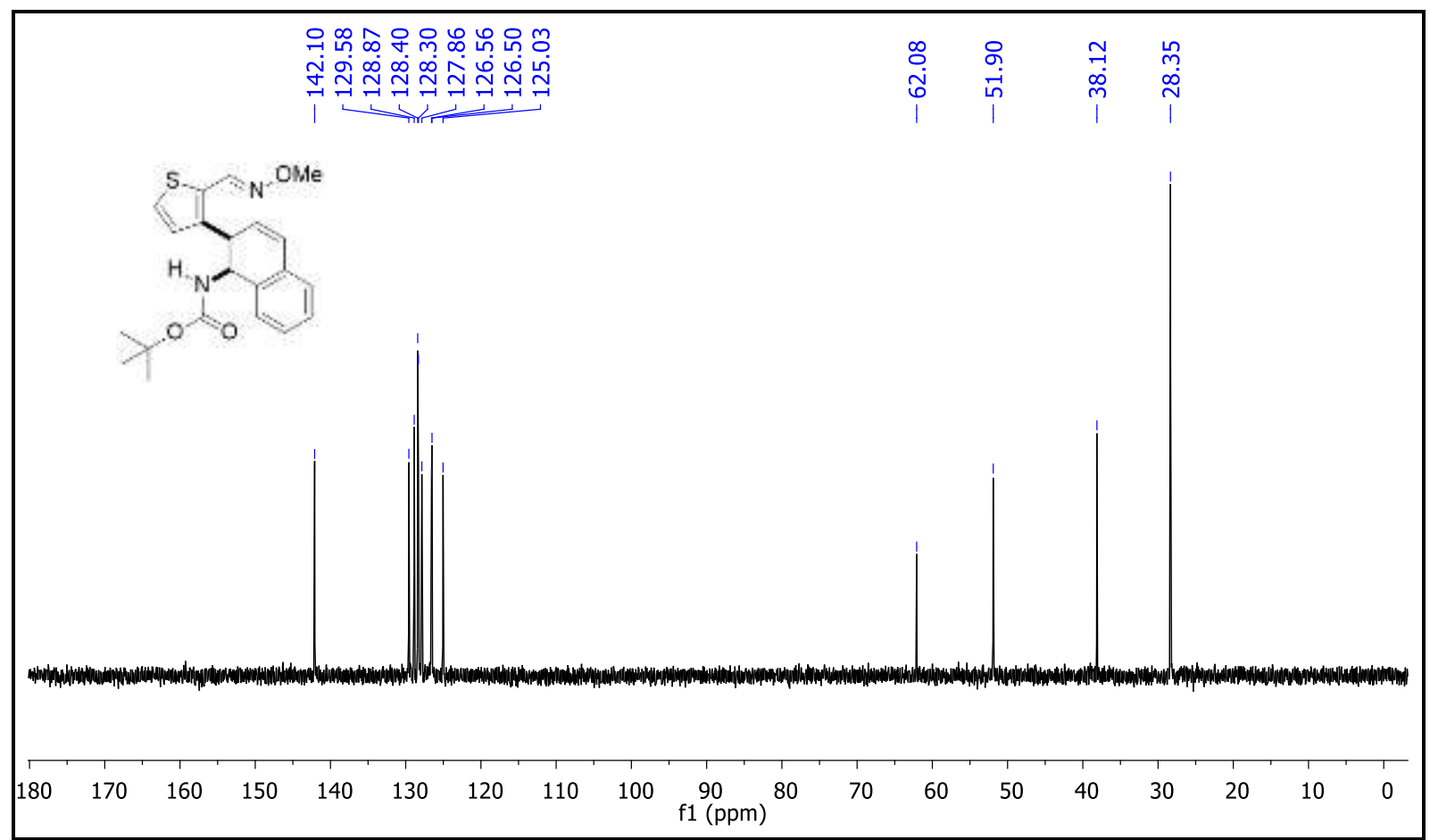


${ }^{1} \mathrm{H}$ and ${ }^{13} \mathrm{C}\left\{{ }^{1} \mathrm{H}\right\}$ NMR Spectra of Compound 3pa $\left(\mathrm{CDCl}_{3}, 400 \mathrm{MHz}\right)$.
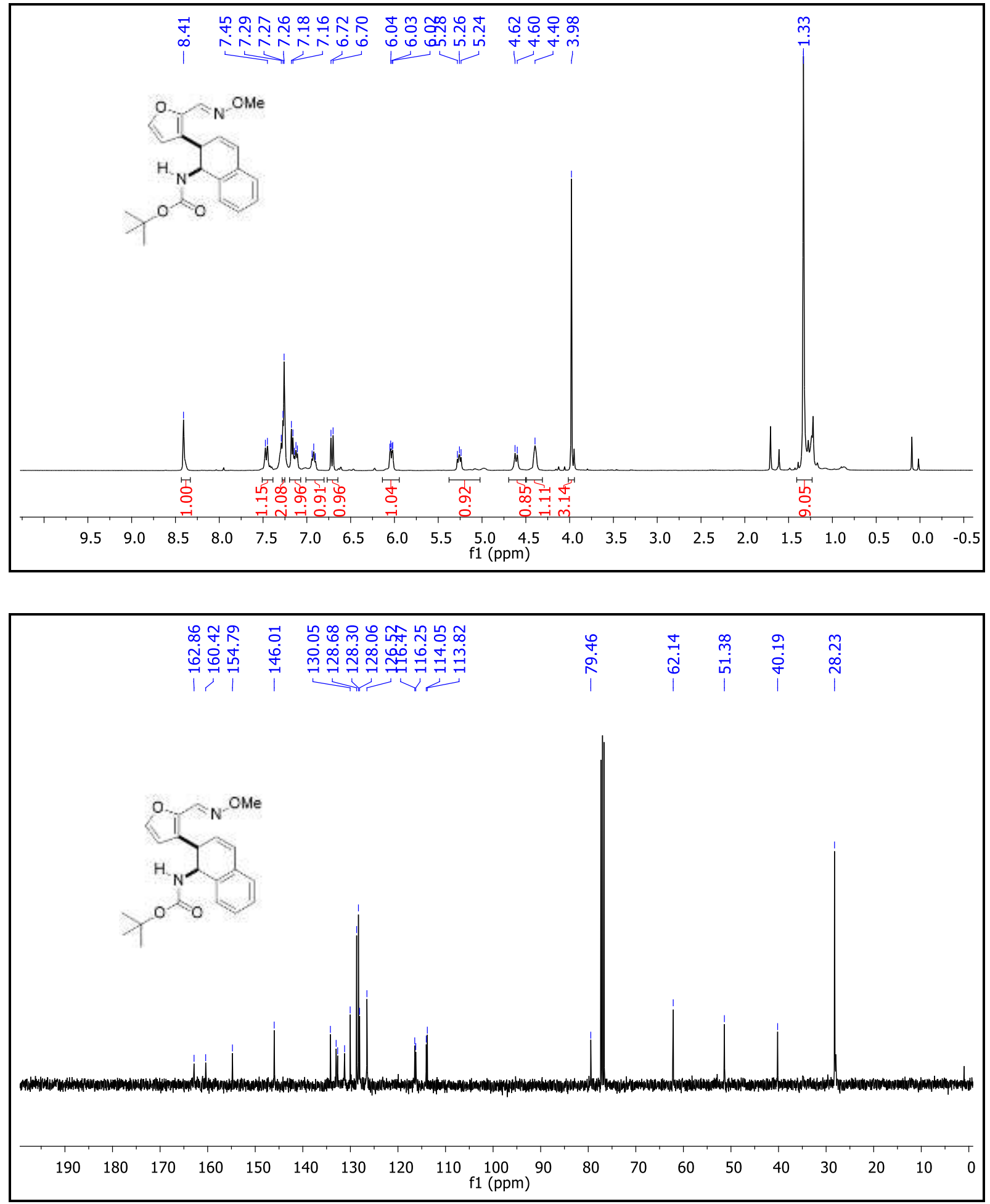
DEPT (135) NMR Spectrum of Compound 3pa $\left(\mathrm{CDCl}_{3}, 101 \mathrm{MHz}\right)$.

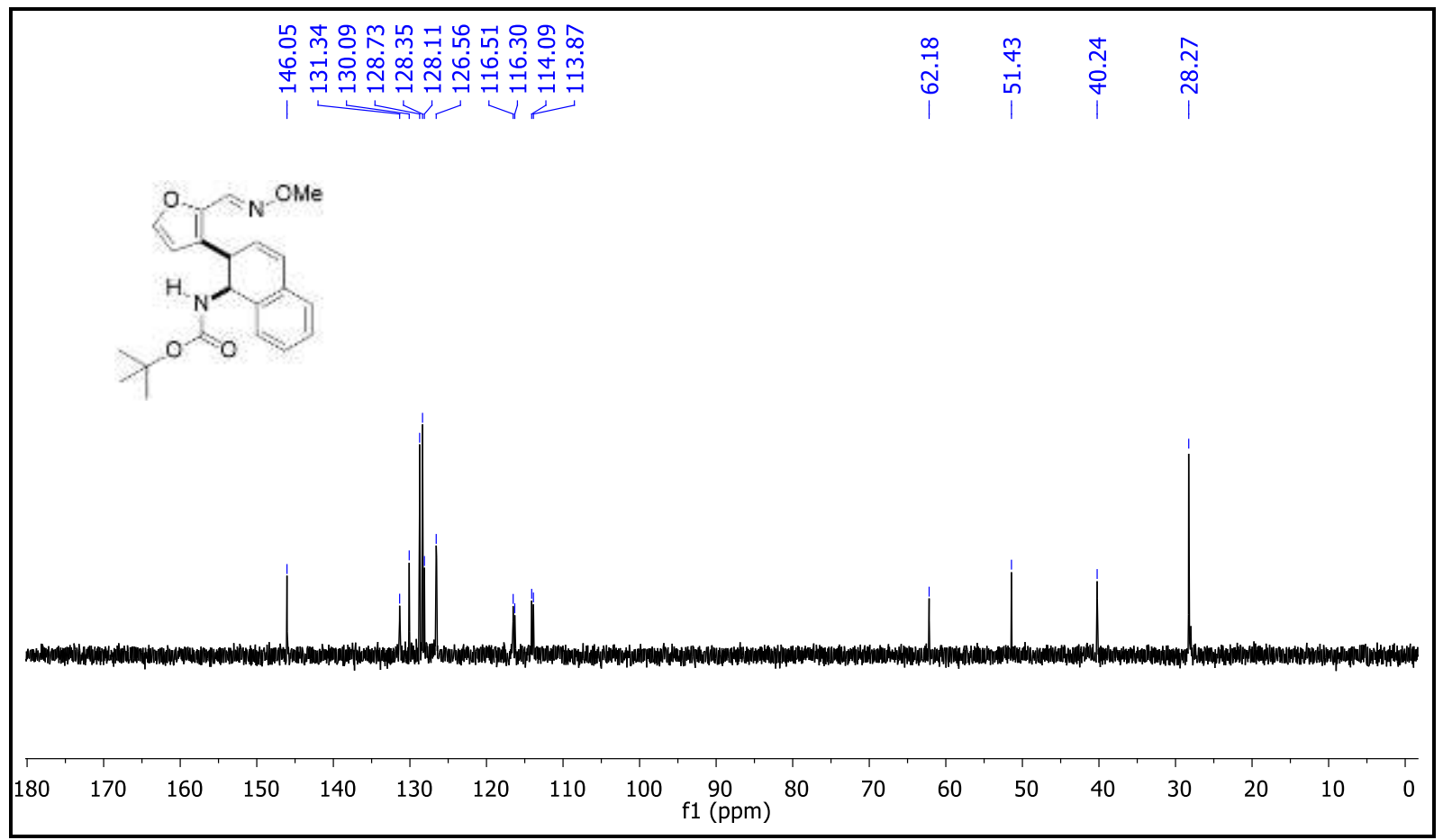


${ }^{1} \mathrm{H}$ and ${ }^{13} \mathrm{C}\left\{{ }^{1} \mathrm{H}\right\}$ NMR Spectra of Compound 3qa $\left(\mathrm{CDCl}_{3}, 400 \mathrm{MHz}\right)$.
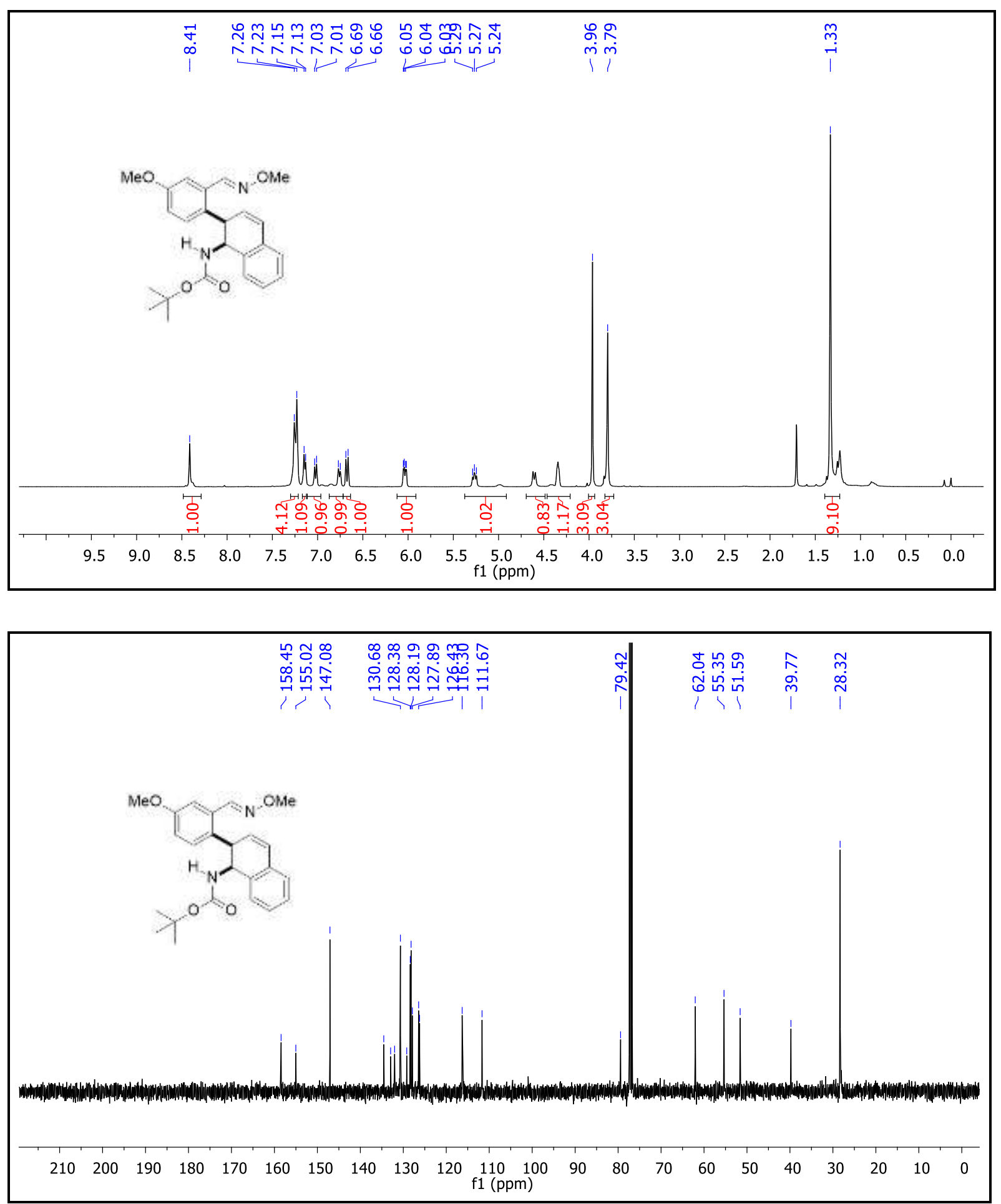
DEPT (135) NMR Spectrum of Compound 3qa ( $\left.\mathrm{CDCl}_{3}, 101 \mathrm{MHz}\right)$.

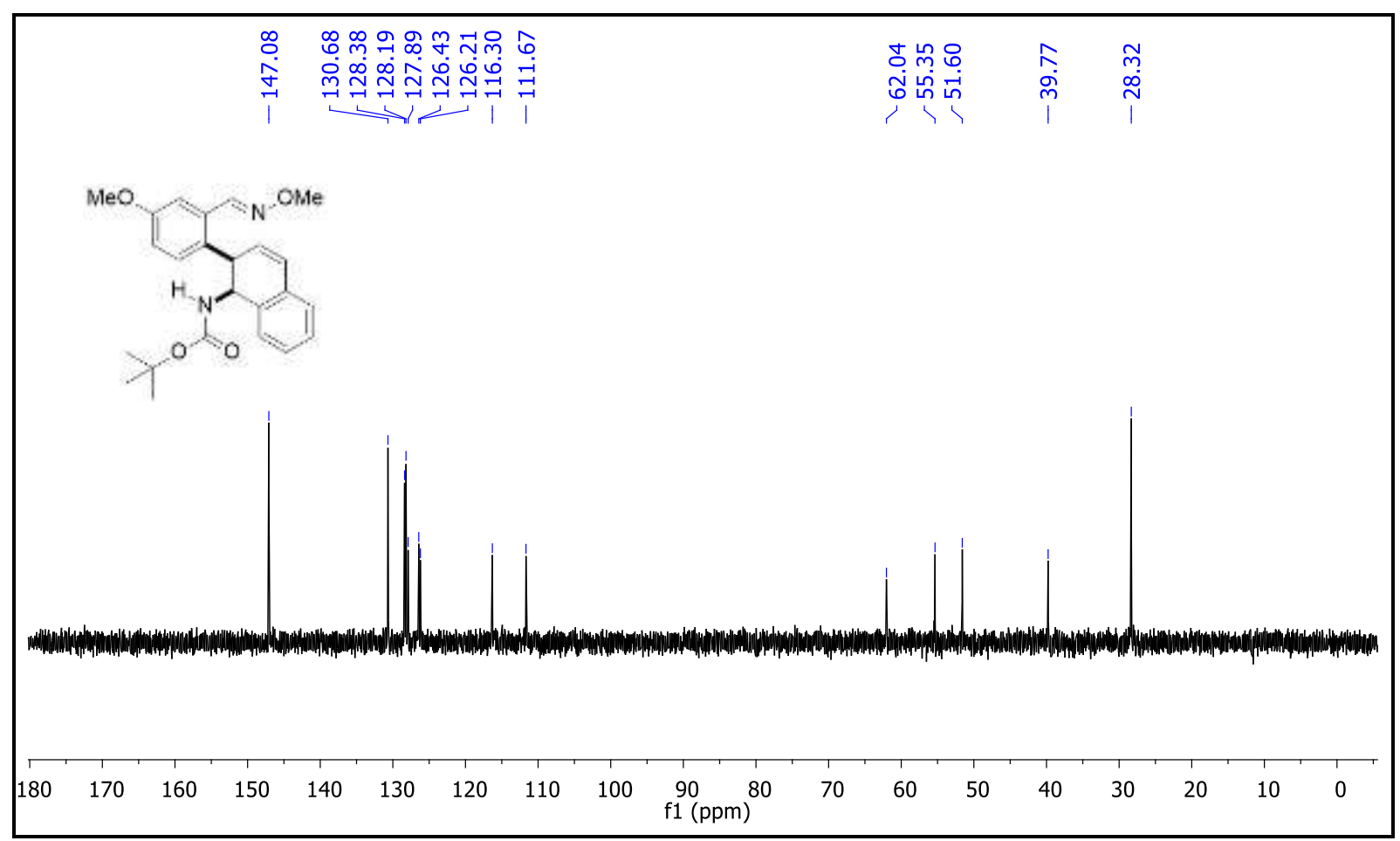


${ }^{1} \mathrm{H}$ and ${ }^{13} \mathrm{C}\left\{{ }^{1} \mathrm{H}\right\}$ NMR Spectra of Compound 3ra $\left(\mathrm{CDCl}_{3}, 400 \mathrm{MHz}\right)$.
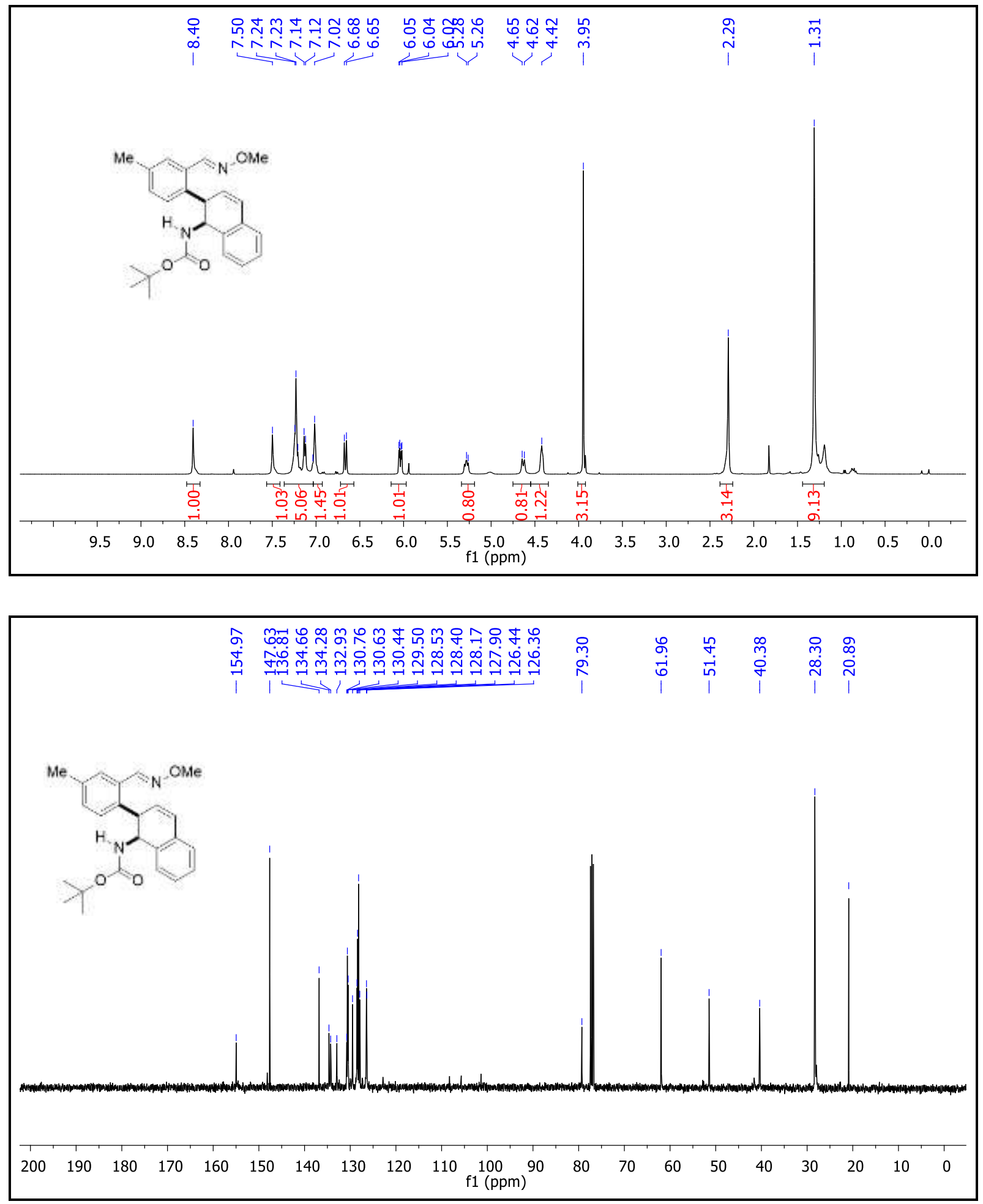
DEPT (135) NMR Spectrum of Compound 3ra $\left(\mathrm{CDCl}_{3}, 101 \mathrm{MHz}\right)$.

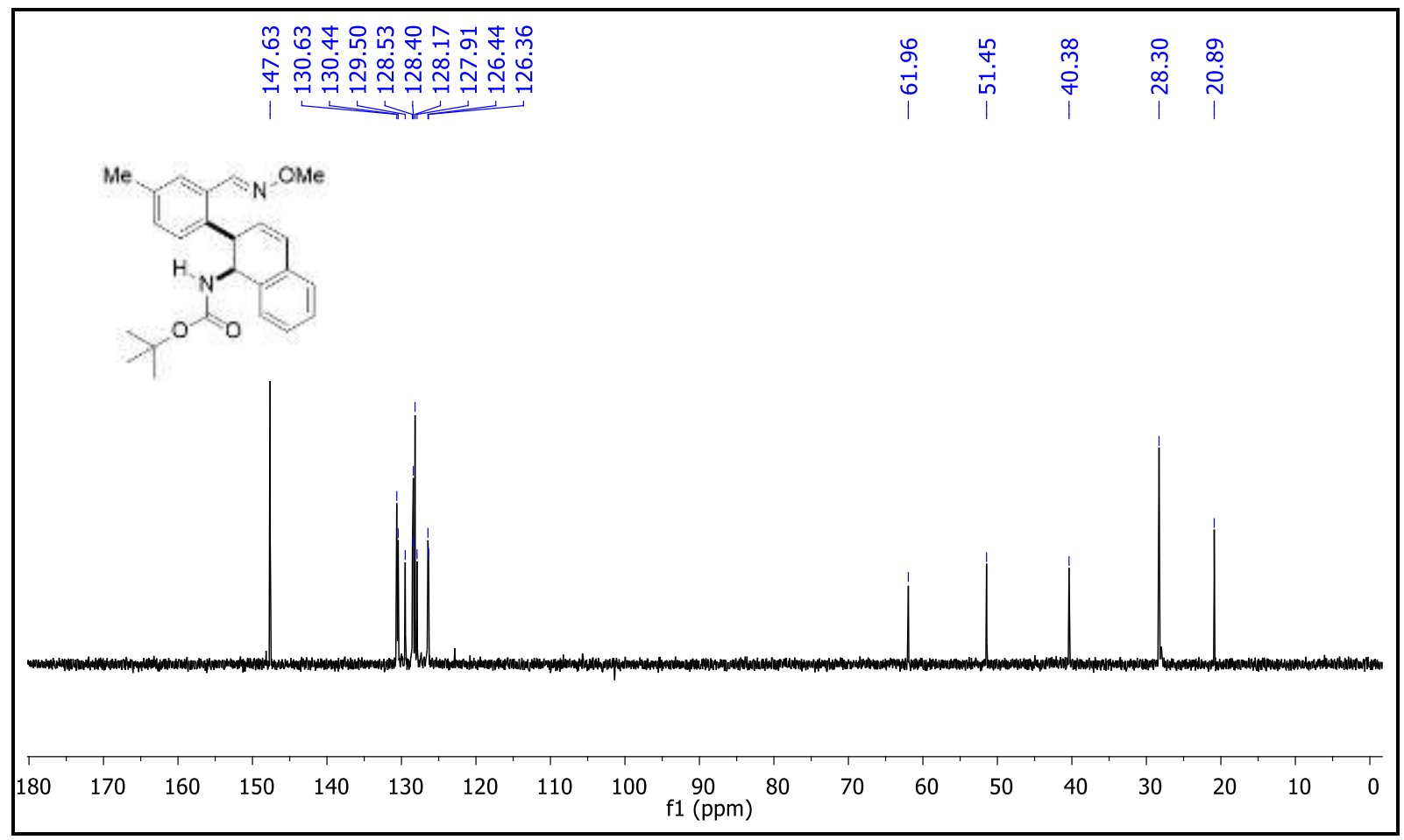


${ }^{1} \mathrm{H}$ and ${ }^{13} \mathrm{C}\left\{{ }^{1} \mathrm{H}\right\}$ NMR Spectra of Compound 3sa $\left(\mathrm{CDCl}_{3}, 400 \mathrm{MHz}\right)$.
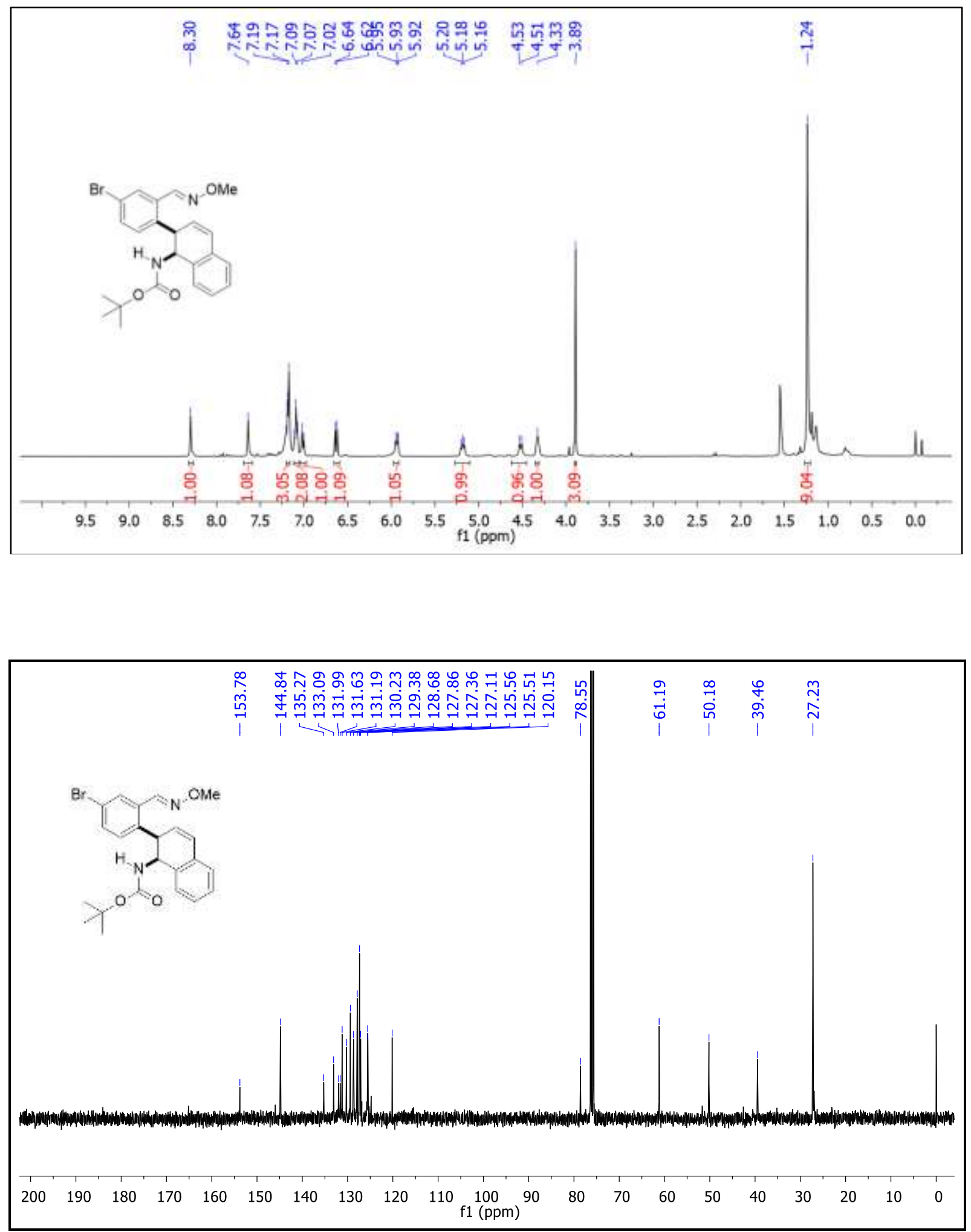
DEPT (135) NMR Spectrum of Compound 3sa ( $\left.\mathrm{CDCl}_{3}, 101 \mathrm{MHz}\right)$.

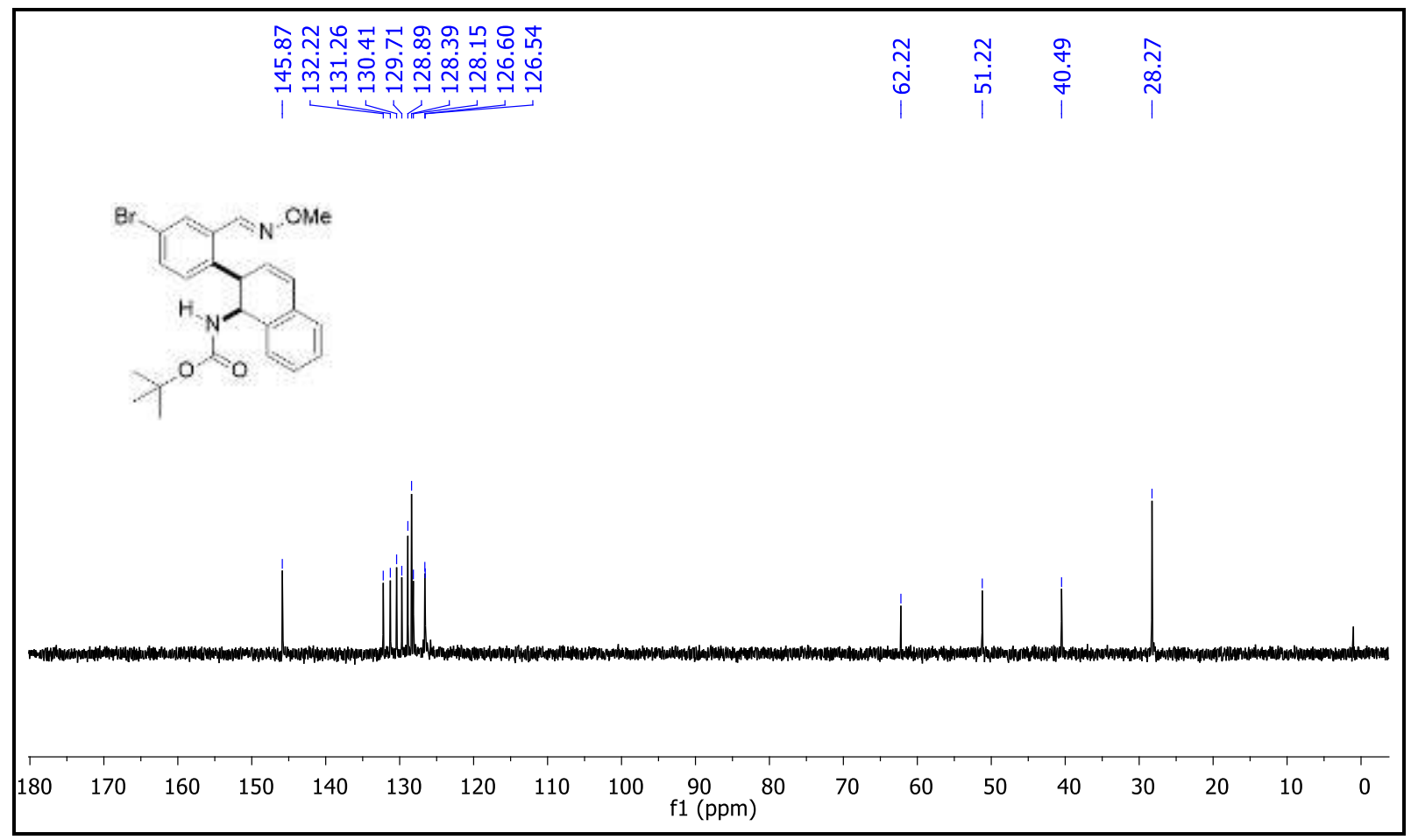


${ }^{1} \mathrm{H}$ and ${ }^{13} \mathrm{C}\left\{{ }^{1} \mathrm{H}\right\}$ NMR Spectra of Compound 3ta $\left(\mathrm{CDCl}_{3}, 400 \mathrm{MHz}\right)$.
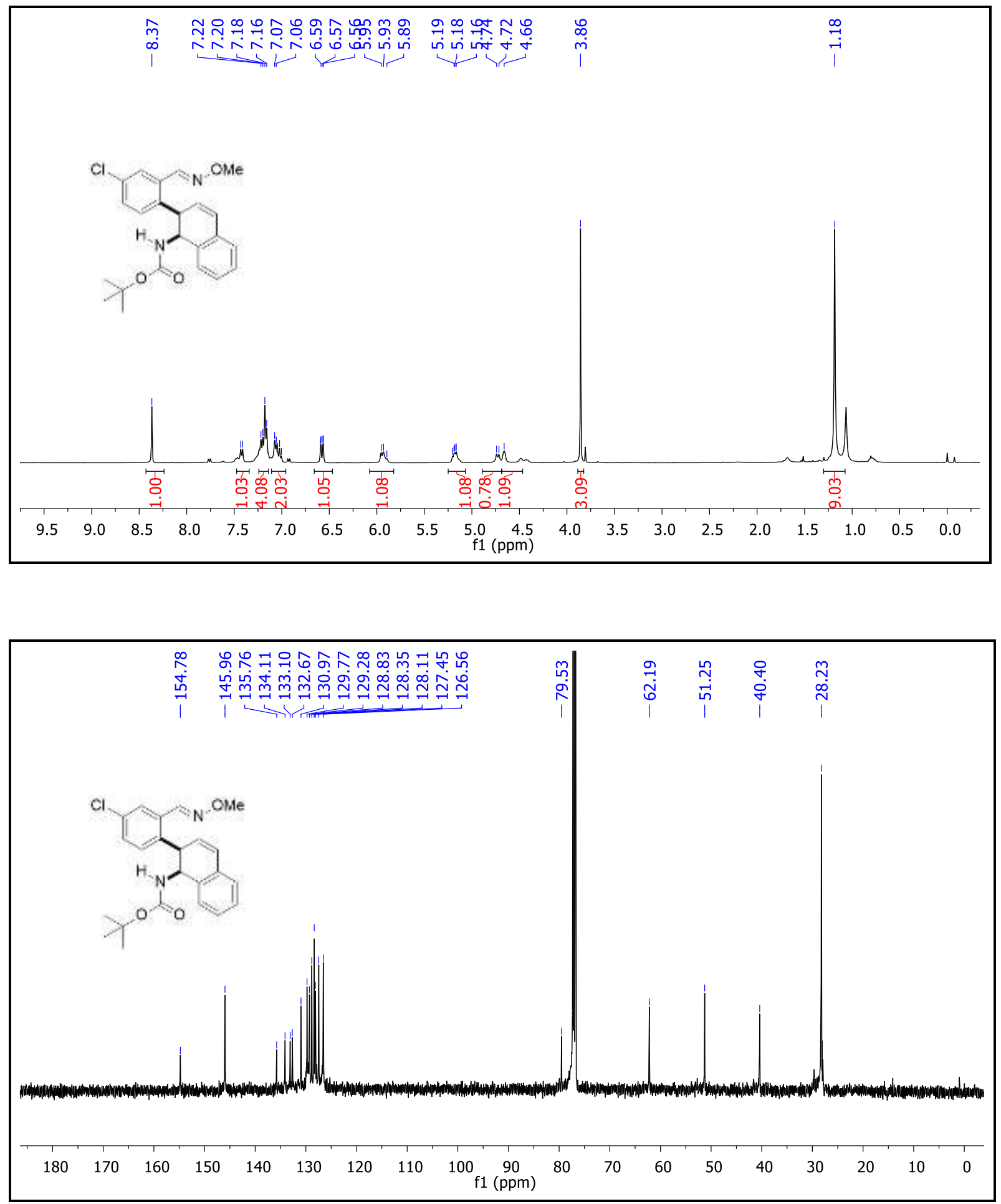
DEPT (135) NMR Spectrum of Compound 3ta $\left(\mathrm{CDCl}_{3}, 101 \mathrm{MHz}\right)$.

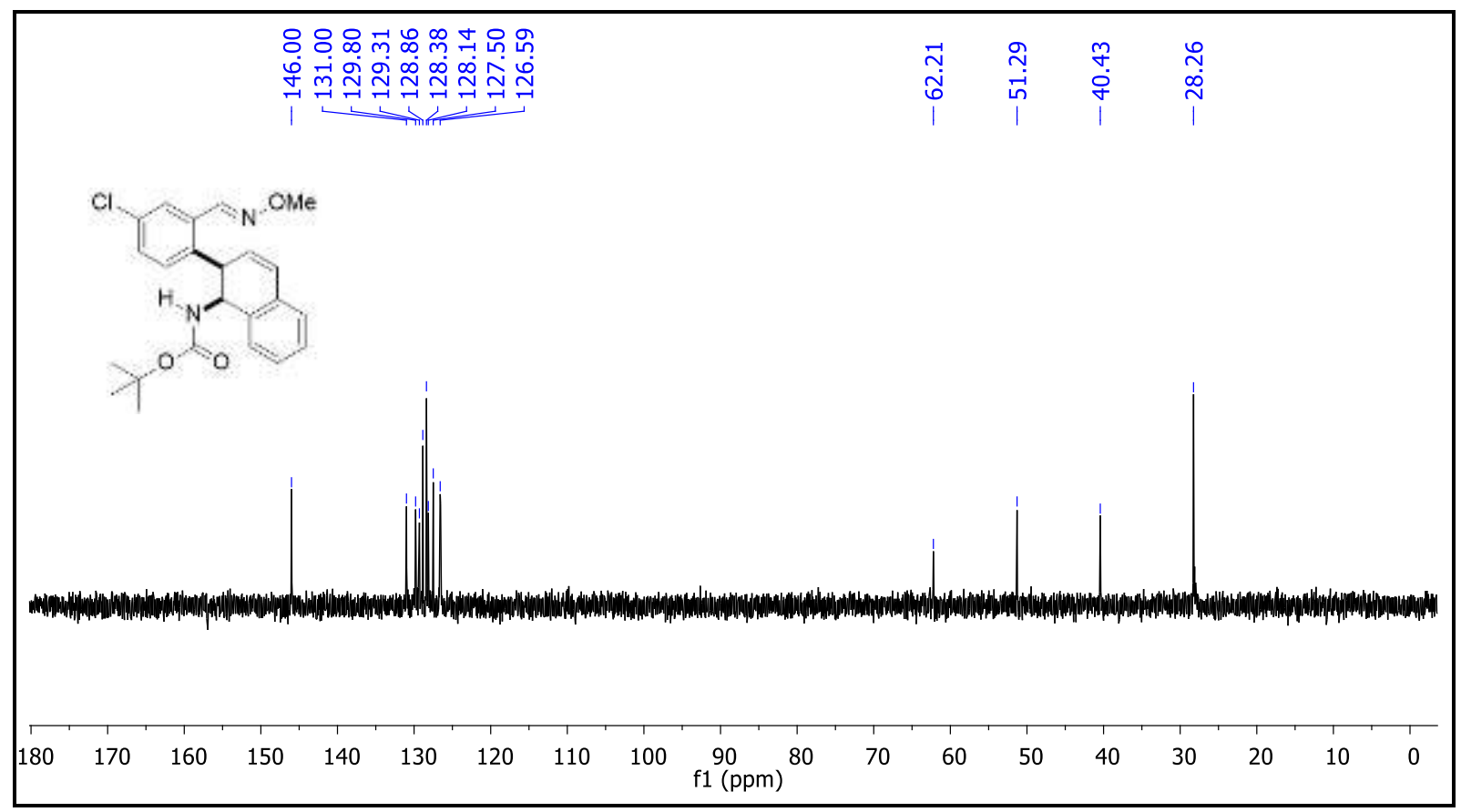


${ }^{1} \mathrm{H}$ and ${ }^{13} \mathrm{C}\left\{{ }^{1} \mathrm{H}\right\}$ NMR Spectra of Compound 3ua $\left(\mathrm{CDCl}_{3}, 400 \mathrm{MHz}\right)$.
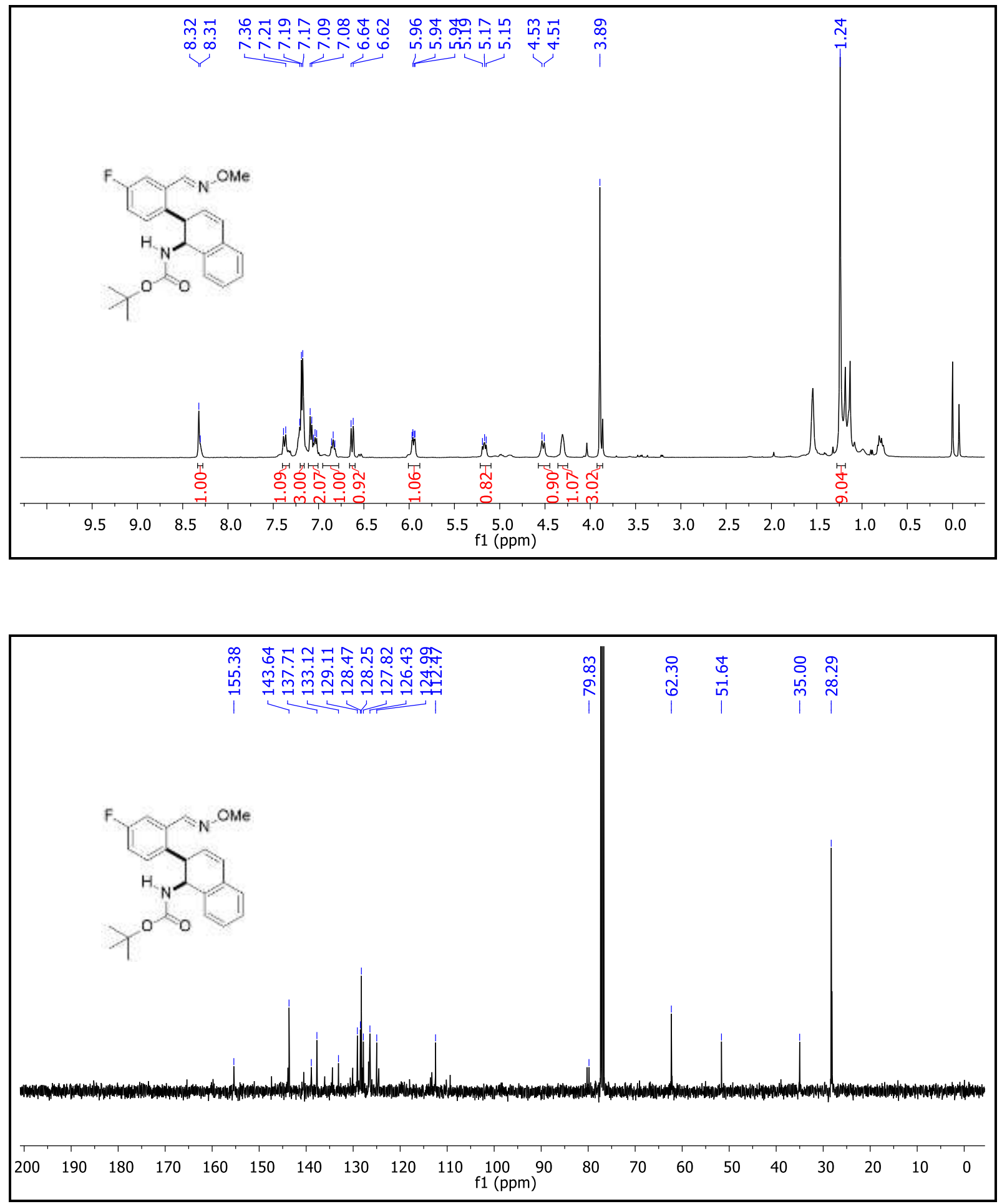
DEPT (135) NMR Spectrum of Compound 3ua $\left(\mathrm{CDCl}_{3}, 101 \mathrm{MHz}\right)$.

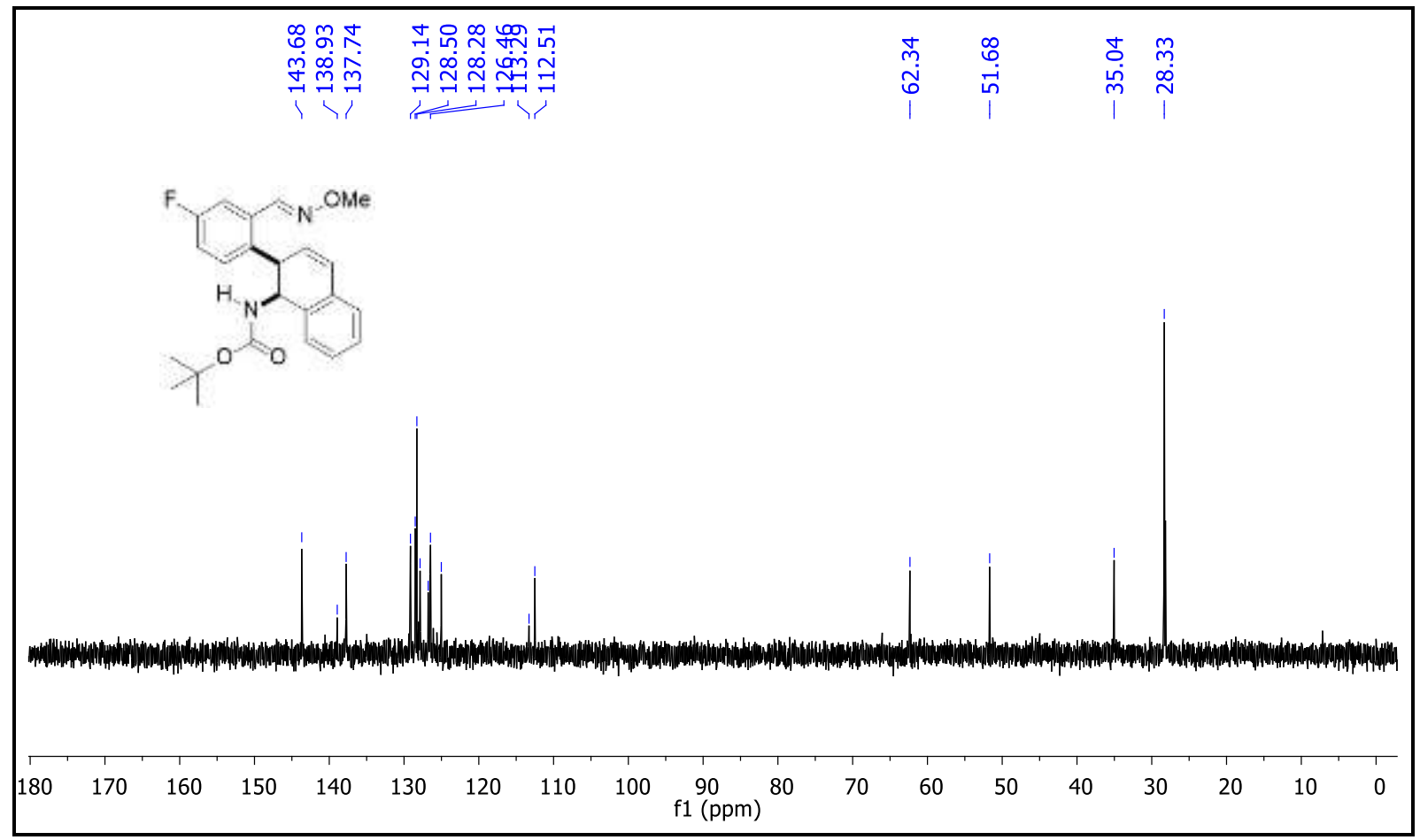

${ }^{19} \mathrm{~F}$ NMR Spectrum of Compound 3ua $\left(\mathrm{CDCl}_{3}, 471 \mathrm{MHz}\right)$.

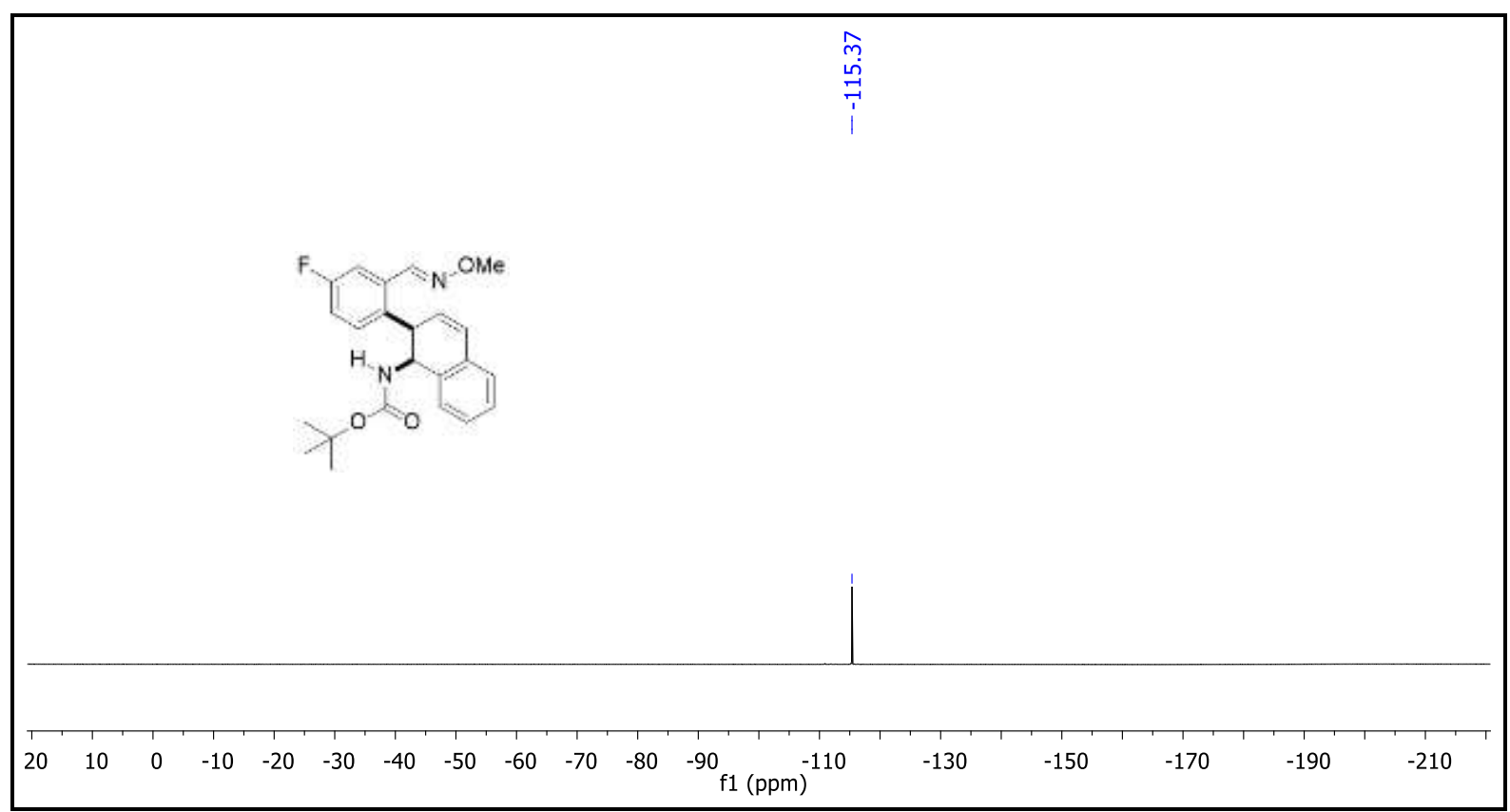


${ }^{1} \mathrm{H}$ and ${ }^{13} \mathrm{C}\left\{{ }^{1} \mathrm{H}\right\}$ NMR Spectra of Compound 3va $\left(\mathrm{CDCl}_{3}, 400 \mathrm{MHz}\right)$.
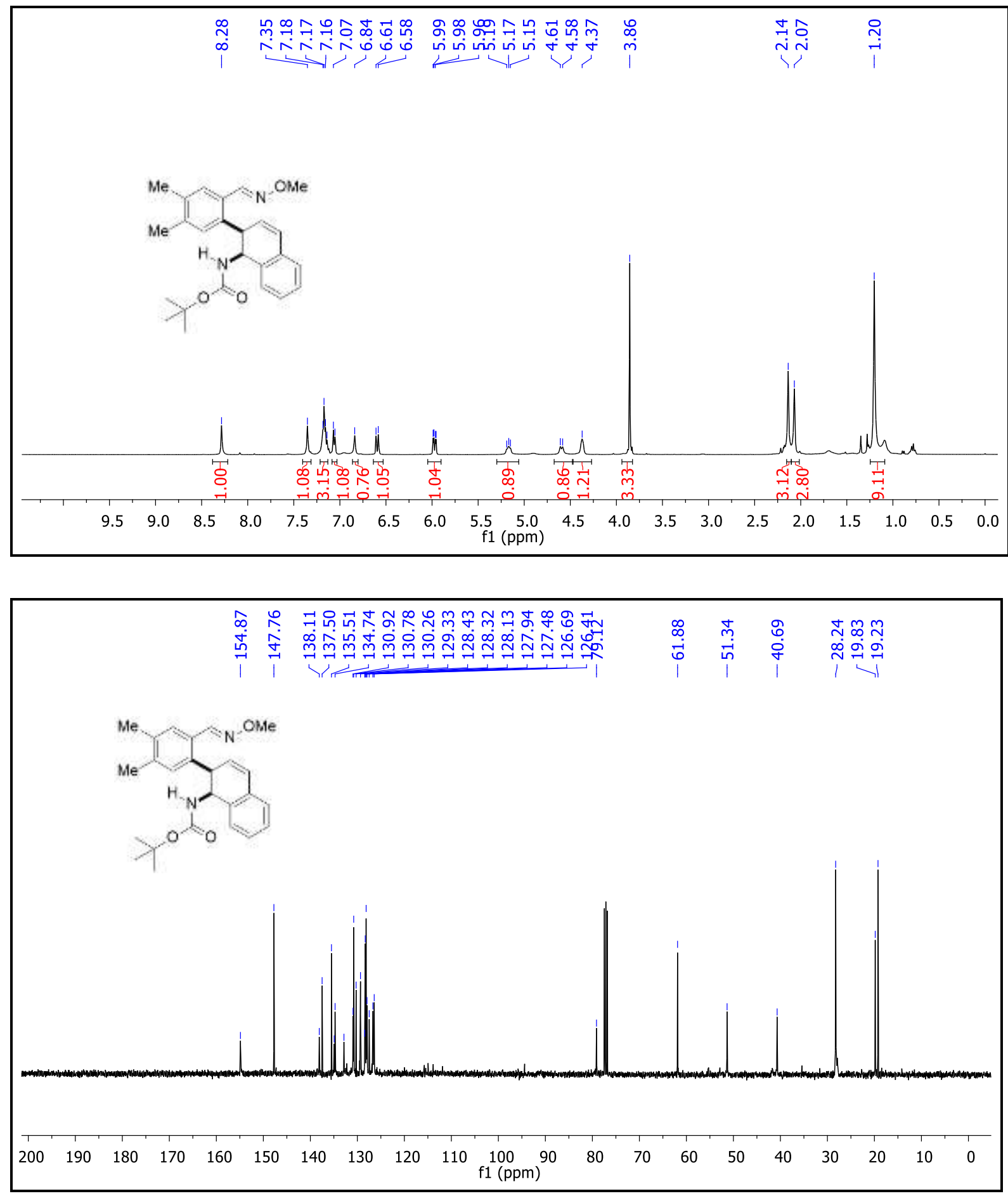
DEPT (135) NMR Spectrum of Compound 3va $\left(\mathrm{CDCl}_{3}, 101 \mathrm{MHz}\right)$.

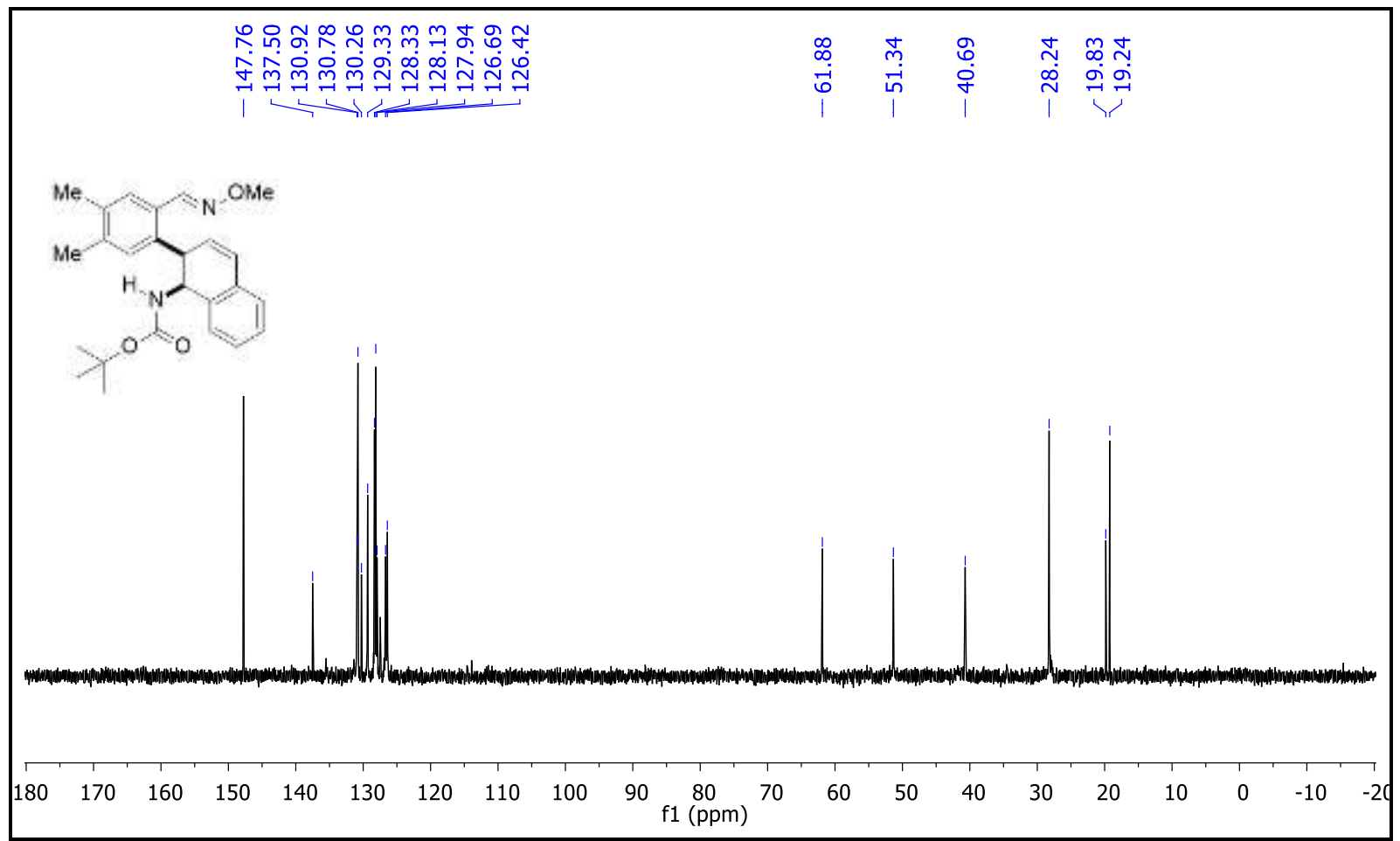


${ }^{1} \mathrm{H}$ and ${ }^{13} \mathrm{C}\left\{{ }^{1} \mathrm{H}\right\}$ NMR Spectra of Compound 3wa $\left(\mathrm{CDCl}_{3}, 400 \mathrm{MHz}\right)$.
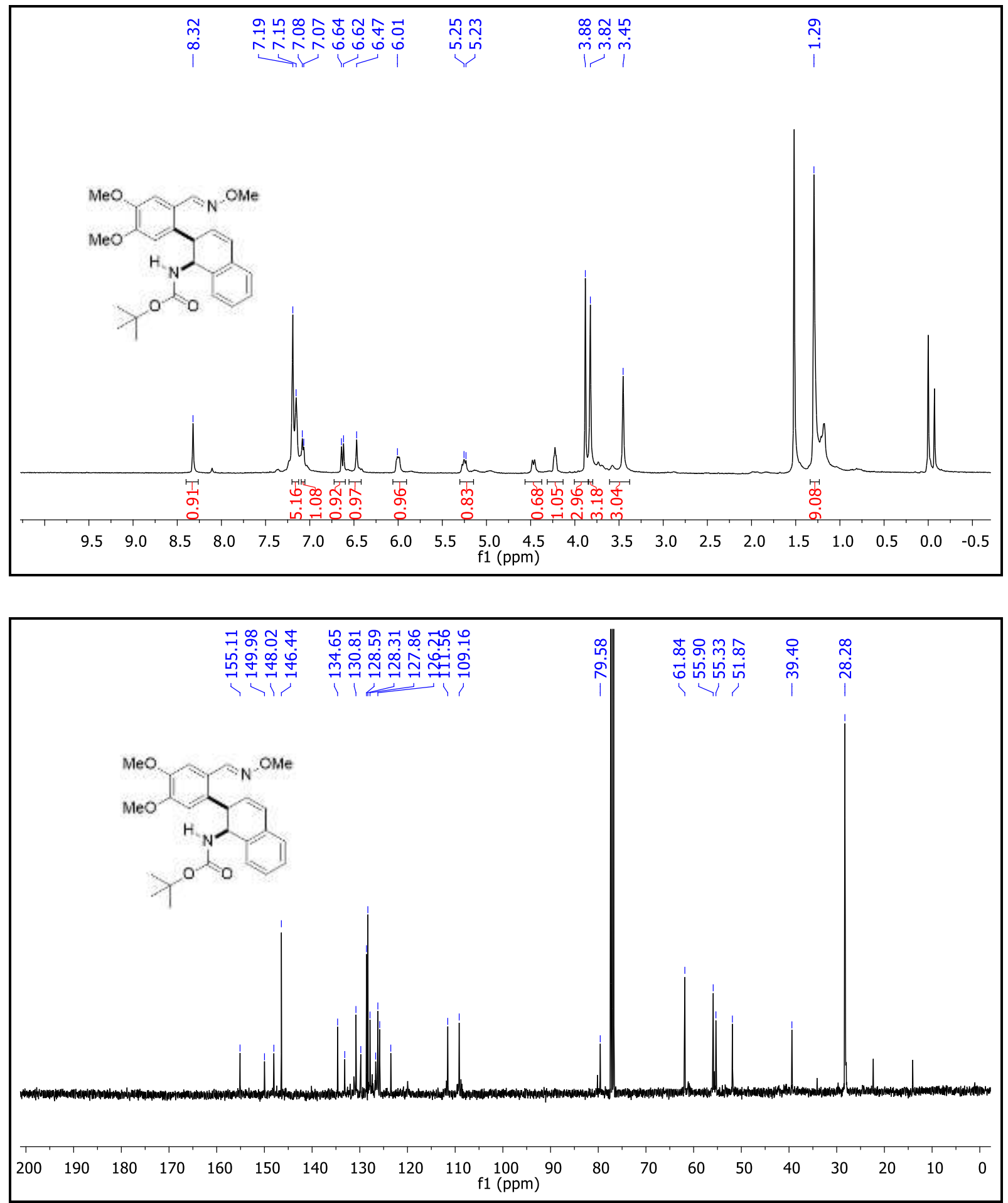
DEPT (135) NMR Spectrum of Compound 3wa $\left(\mathrm{CDCl}_{3}, 101 \mathrm{MHz}\right)$.

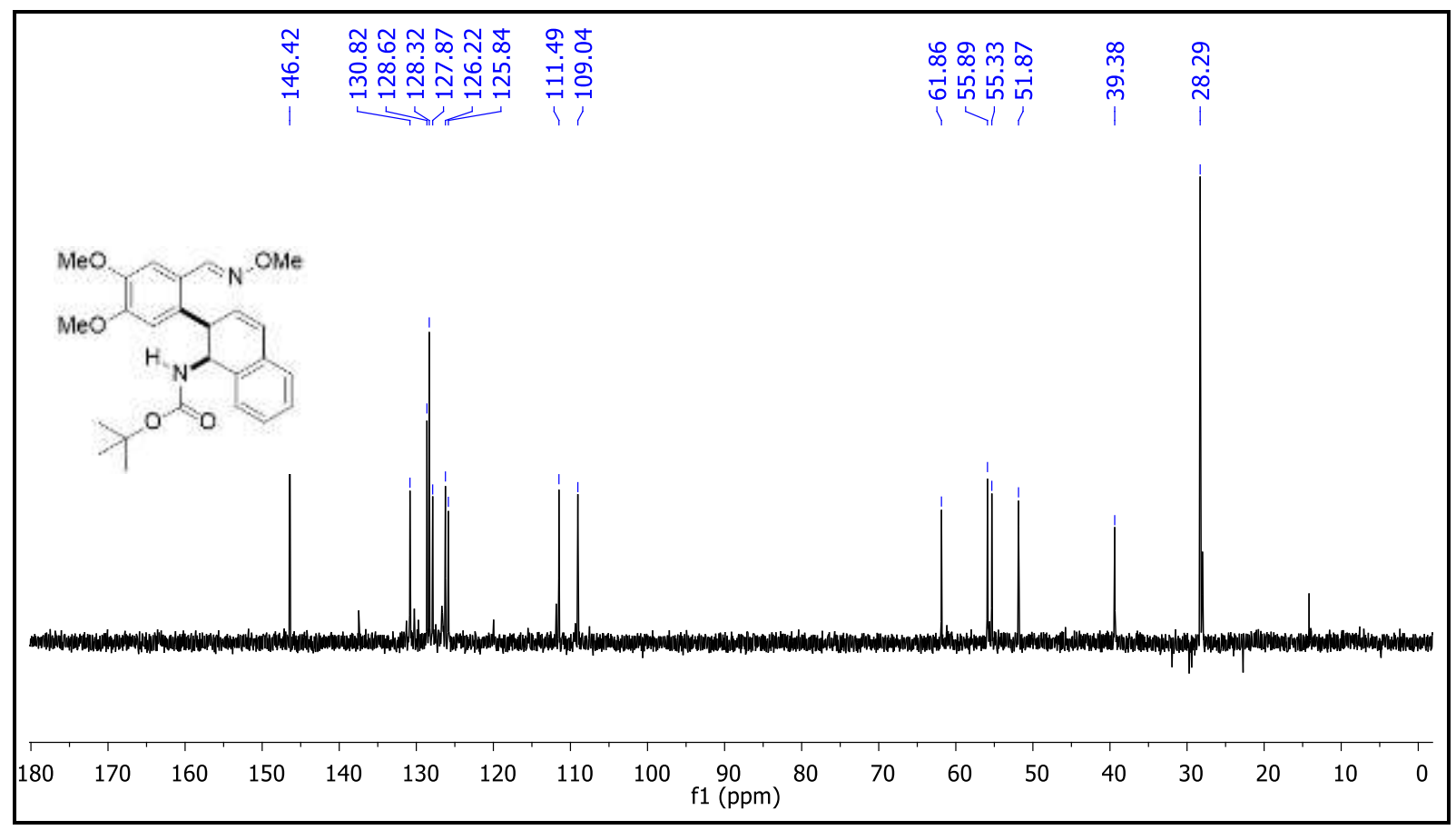


${ }^{1} \mathrm{H}$ and ${ }^{13} \mathrm{C}\left\{{ }^{1} \mathrm{H}\right\}$ NMR Spectra of Compound 3xa $\left(\mathrm{CDCl}_{3}, 400 \mathrm{MHz}\right)$.
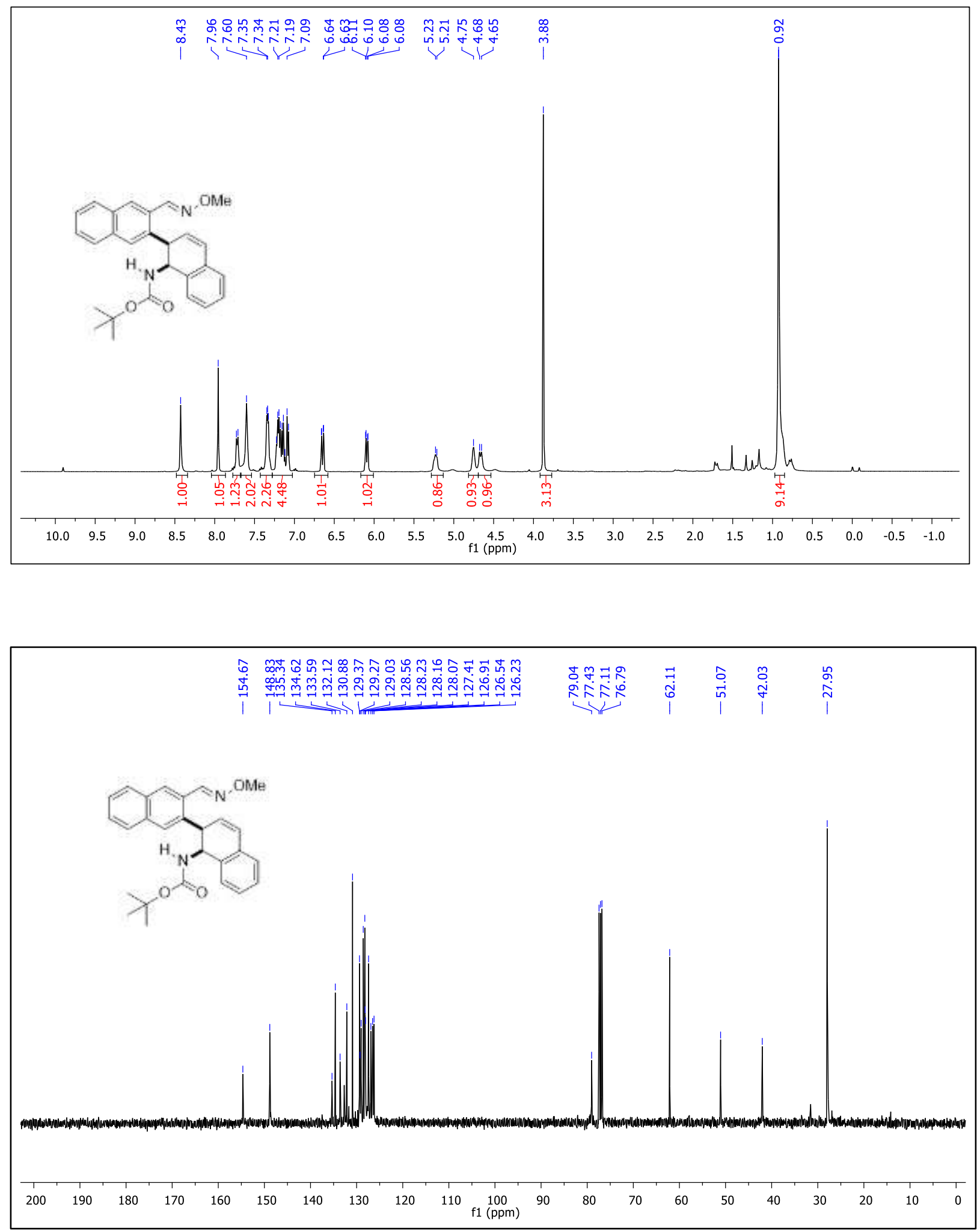
DEPT (135) NMR Spectrum of Compound 3xa $\left(\mathrm{CDCl}_{3}, 101 \mathrm{MHz}\right)$.

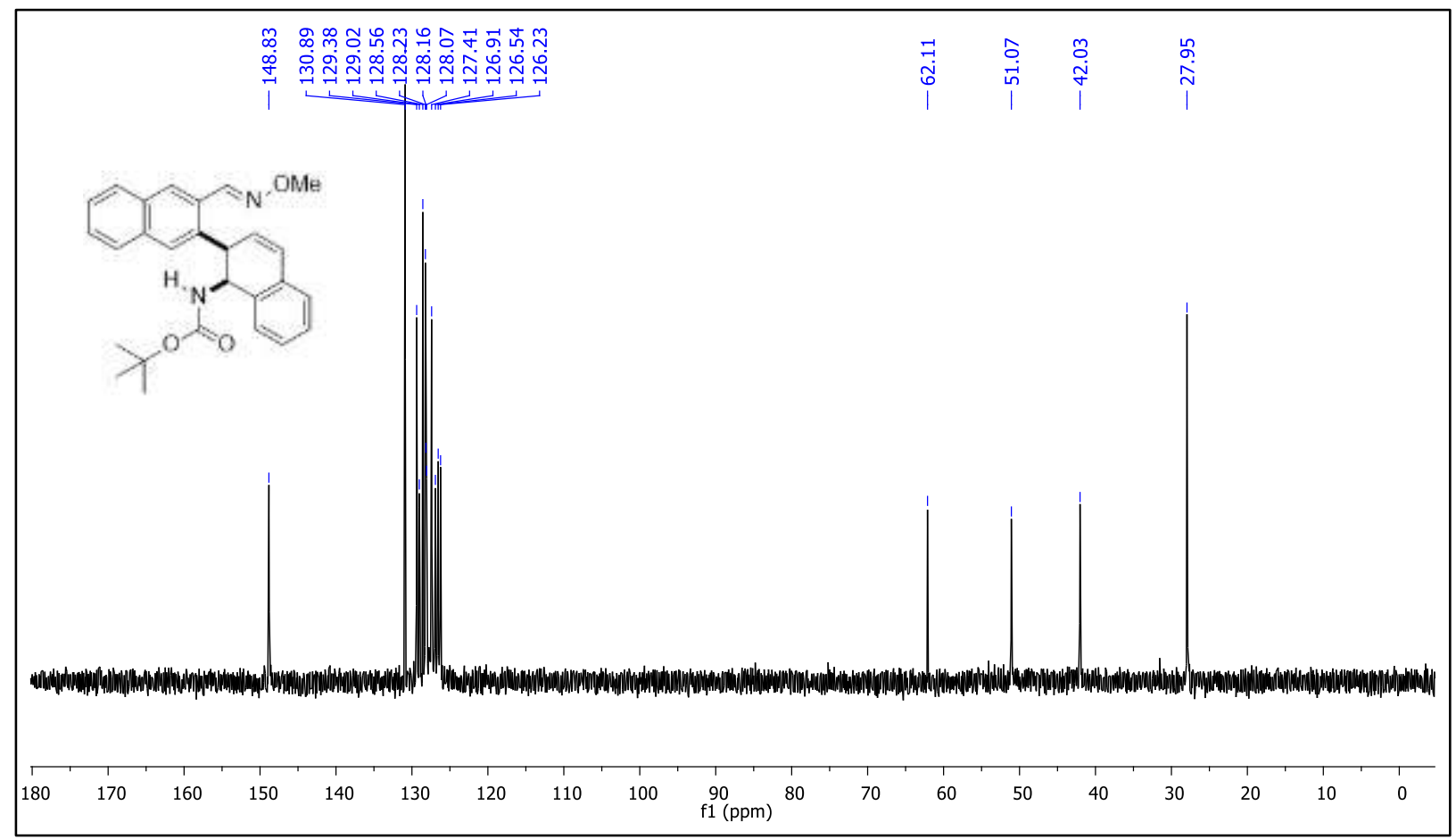


${ }^{1} \mathrm{H}$ and ${ }^{13} \mathrm{C}\left\{{ }^{1} \mathrm{H}\right\}$ NMR Spectra of Compound 3ya $\left(\mathrm{CDCl}_{3}, 400 \mathrm{MHz}\right)$.
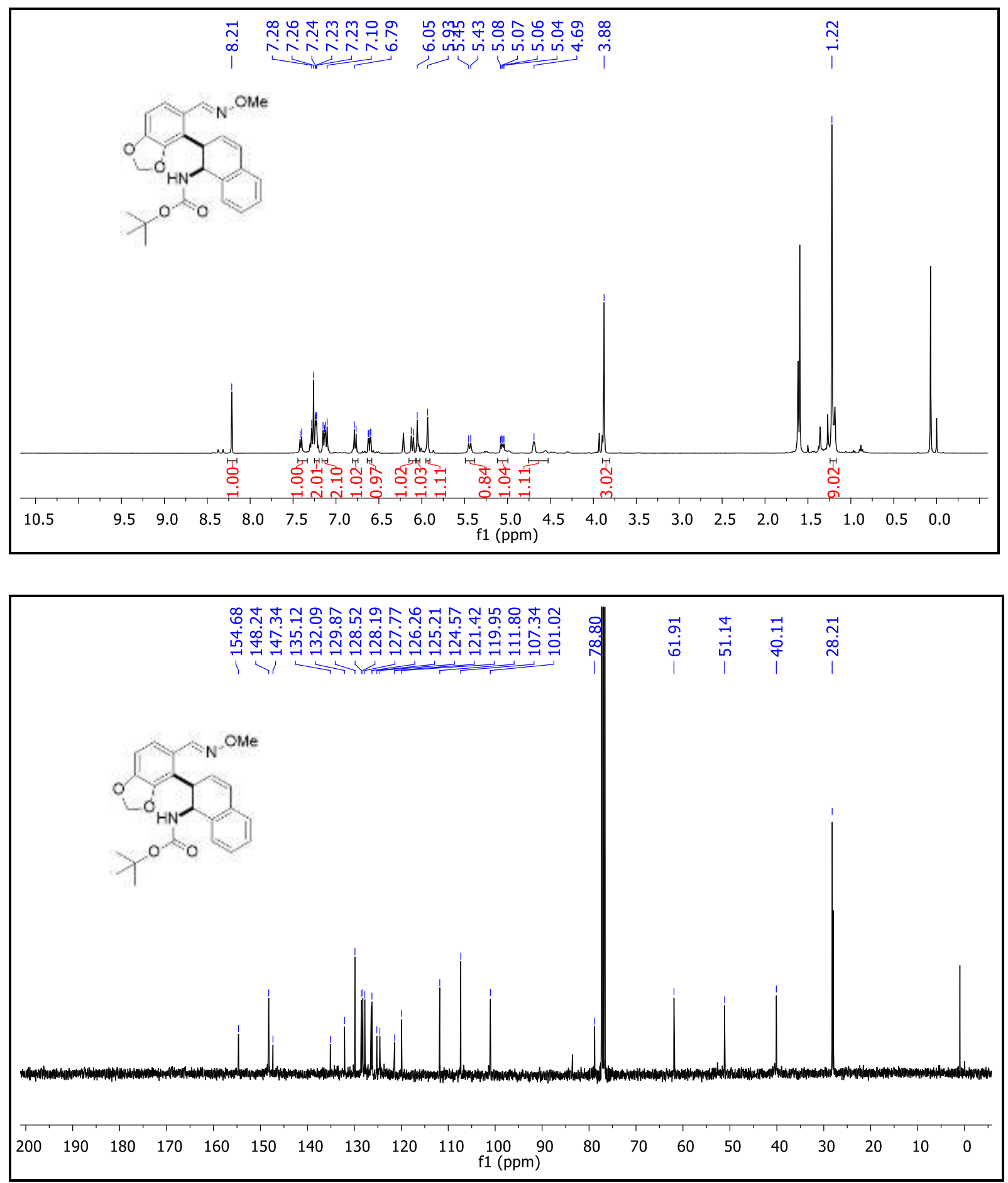
DEPT (135) NMR Spectrum of Compound 3ya $\left(\mathrm{CDCl}_{3}, 101 \mathrm{MHz}\right)$.

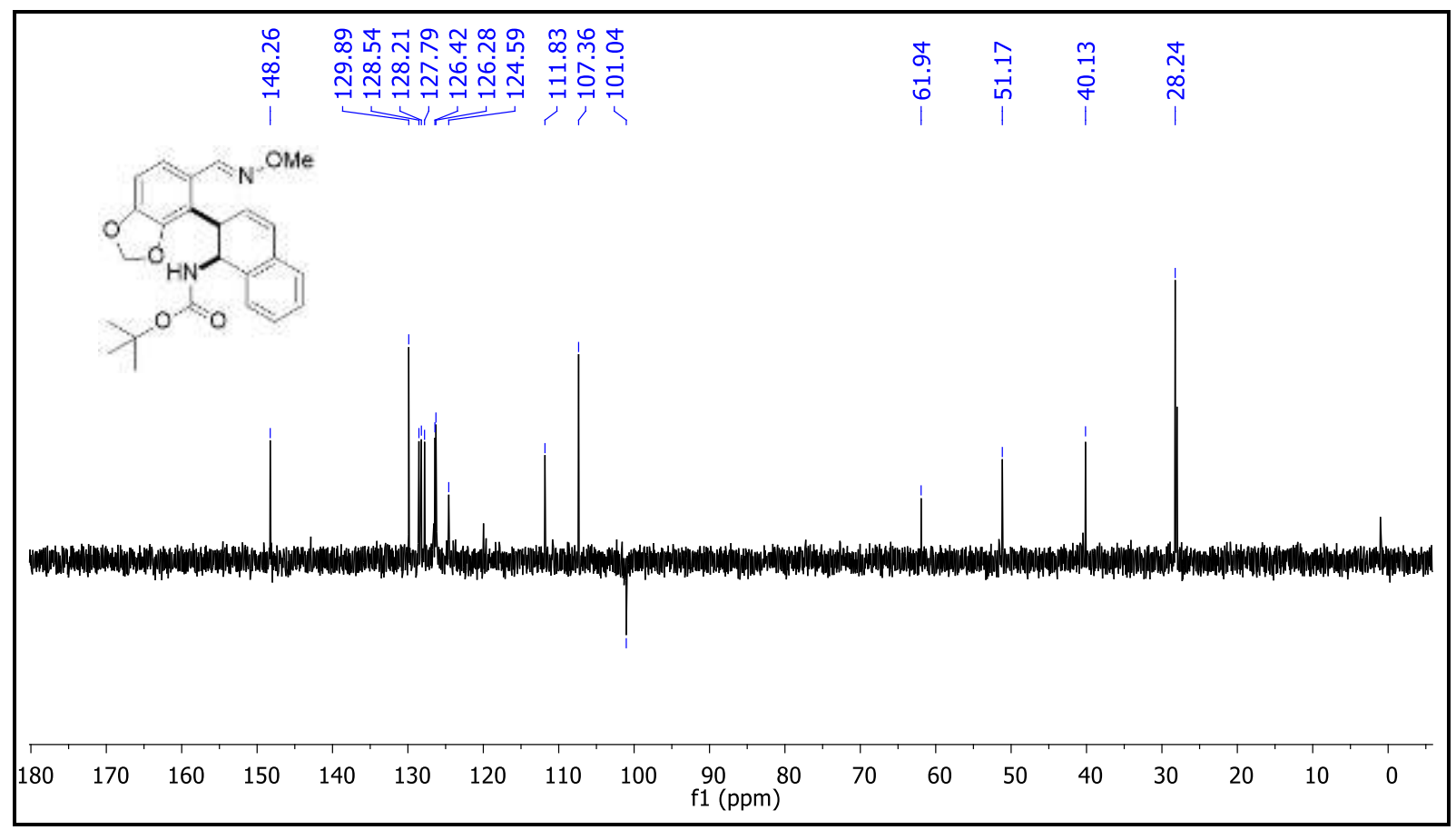


${ }^{1} \mathrm{H}$ and ${ }^{13} \mathrm{C}\left\{{ }^{1} \mathrm{H}\right\}$ NMR Spectra of Compound 3ab $\left(\mathrm{CDCl}_{3}, 400 \mathrm{MHz}\right)$.
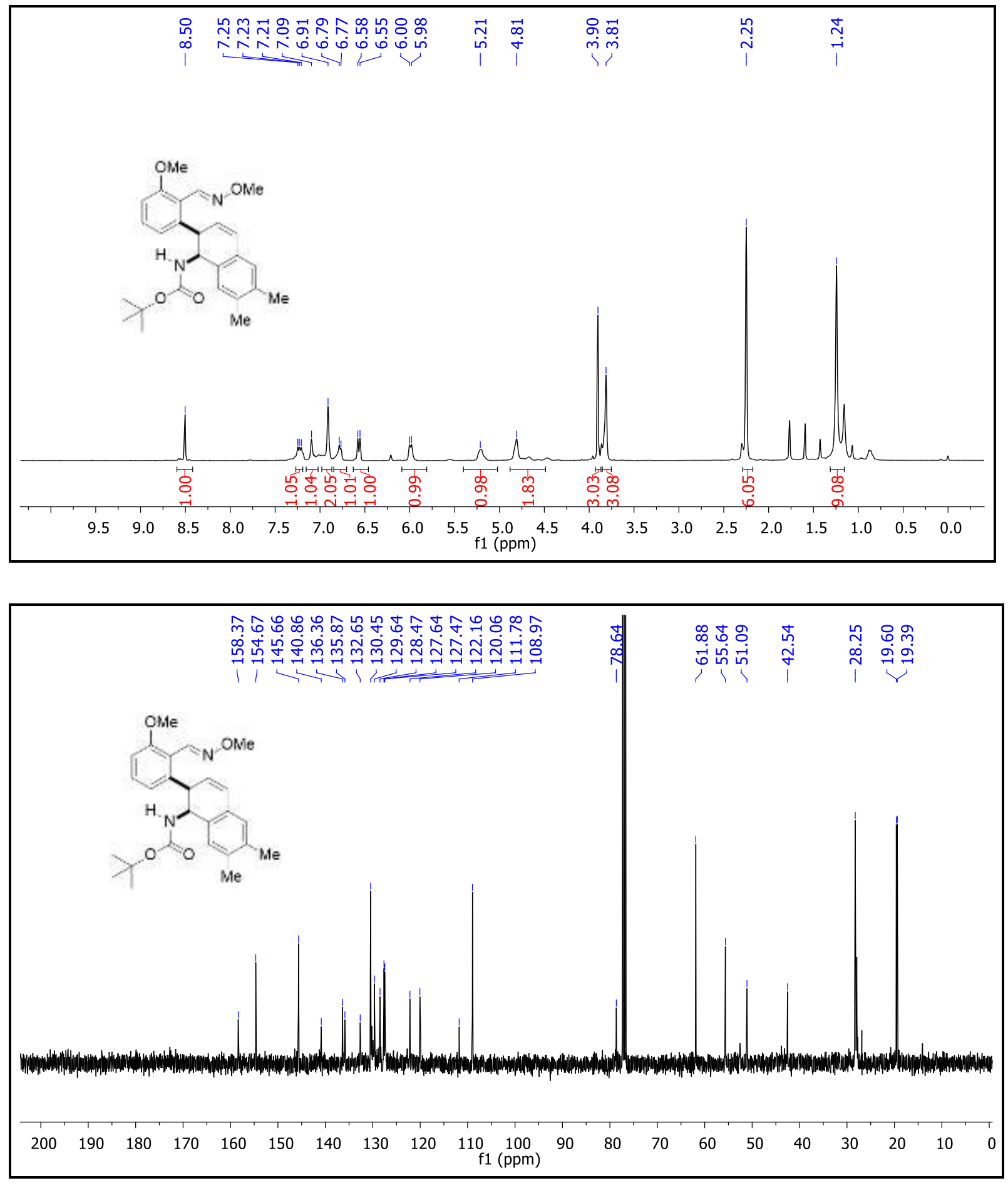
DEPT (135) NMR Spectrum of Compound 3ab $\left(\mathrm{CDCl}_{3}, 101 \mathrm{MHz}\right)$.

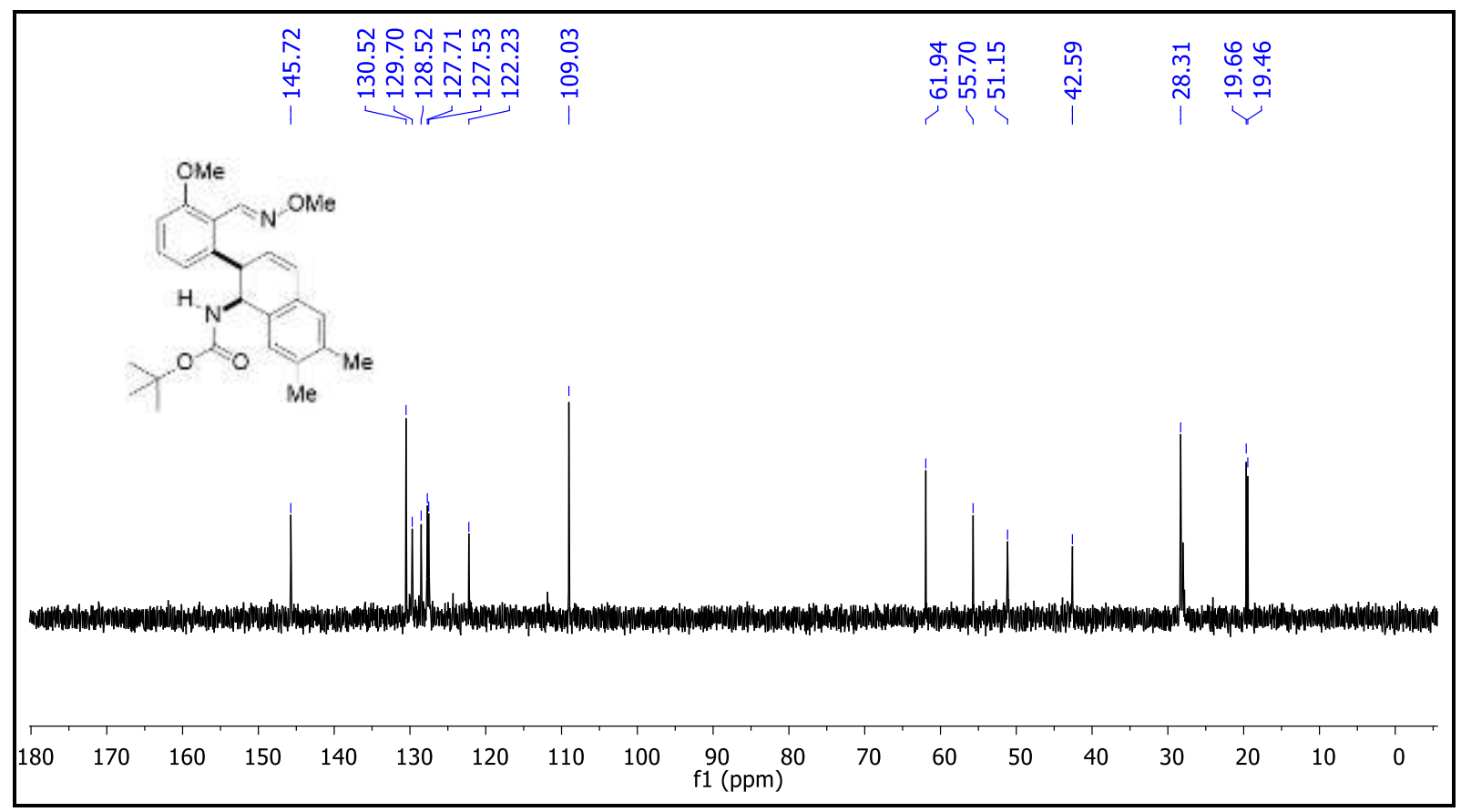


${ }^{1} \mathrm{H}$ and ${ }^{13} \mathrm{C}\left\{{ }^{1} \mathrm{H}\right\}$ NMR Spectra of Compound 3ac $\left(\mathrm{CDCl}_{3}, 400 \mathrm{MHz}\right)$.
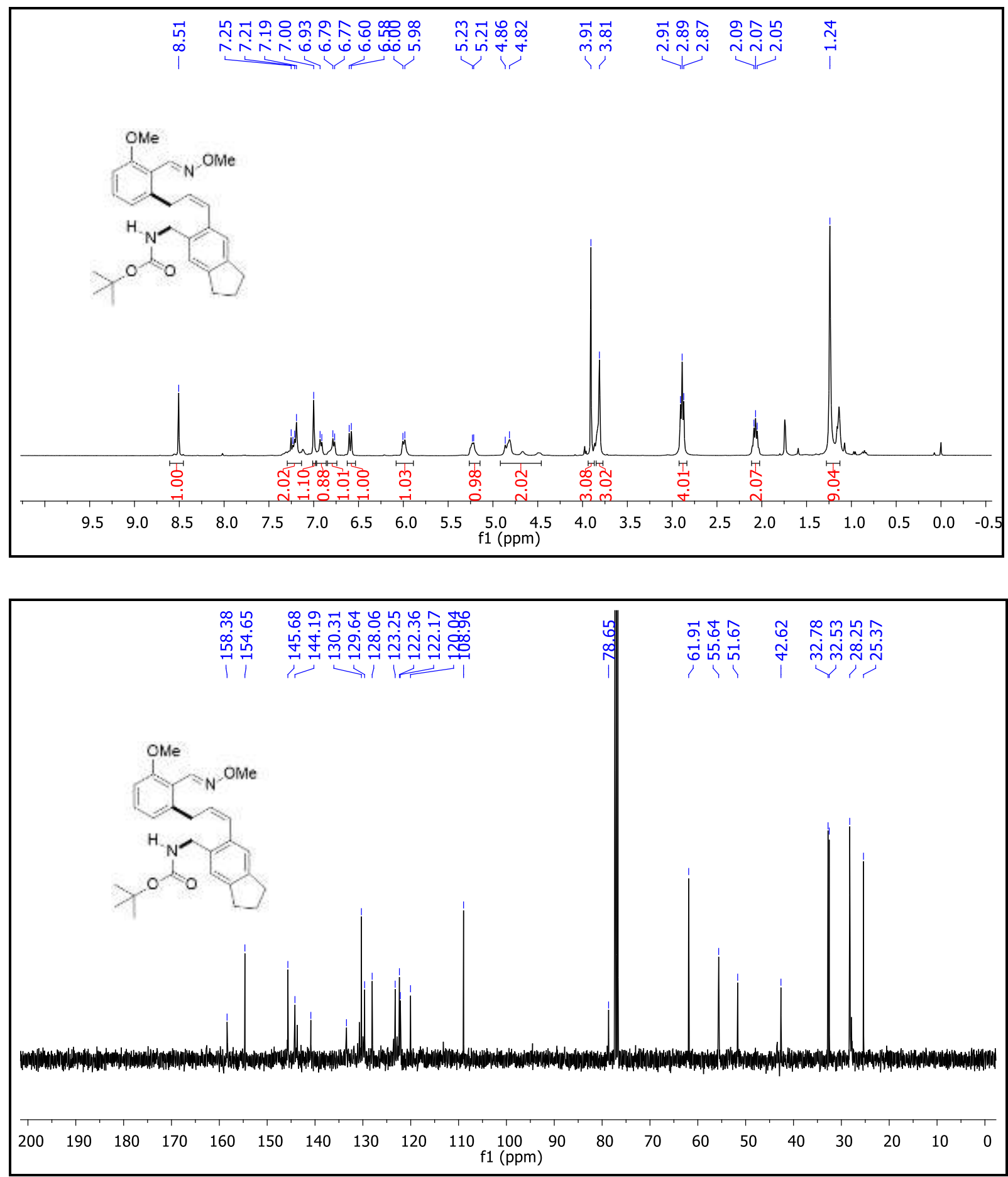
DEPT (135) NMR Spectrum of Compound 3ac $\left(\mathrm{CDCl}_{3}, 101 \mathrm{MHz}\right)$.

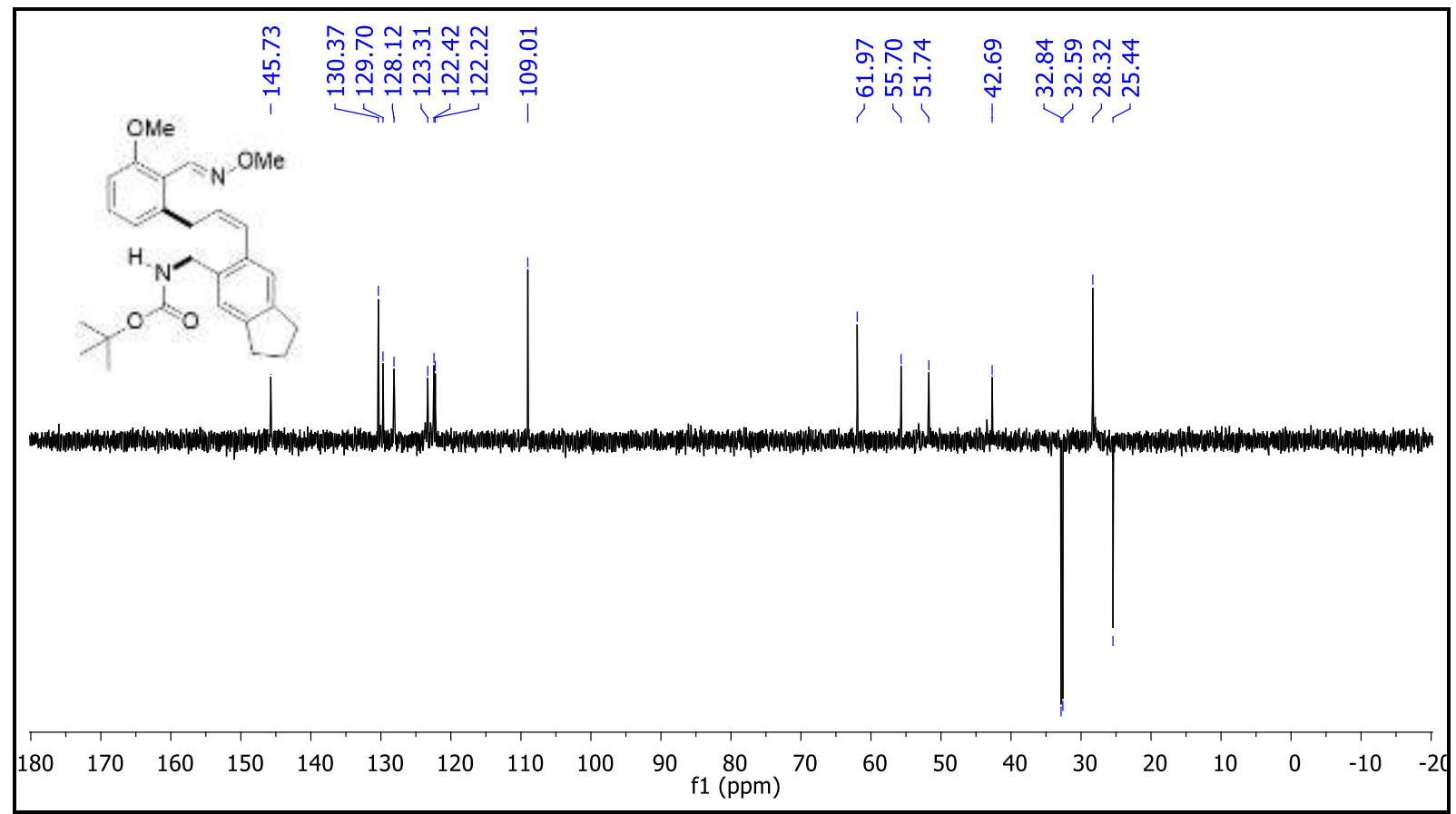


${ }^{1} \mathrm{H}$ and ${ }^{13} \mathrm{C}\left\{{ }^{1} \mathrm{H}\right\}$ NMR Spectra of Compound 3gc $\left(\mathrm{CDCl}_{3}, 400 \mathrm{MHz}\right)$.
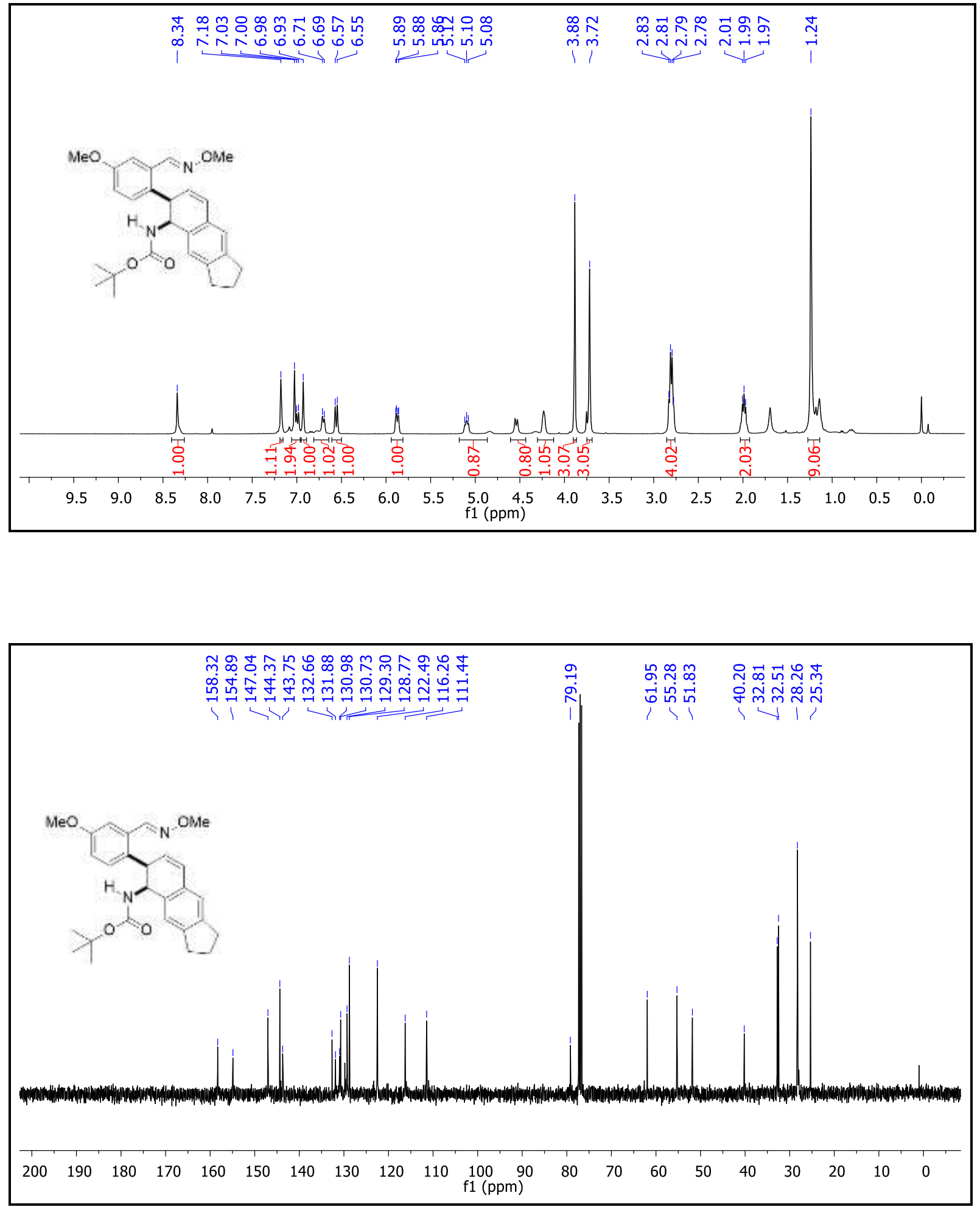
DEPT (135) NMR Spectrum of Compound $3 \mathbf{g c}\left(\mathrm{CDCl}_{3}, 101 \mathrm{MHz}\right)$.

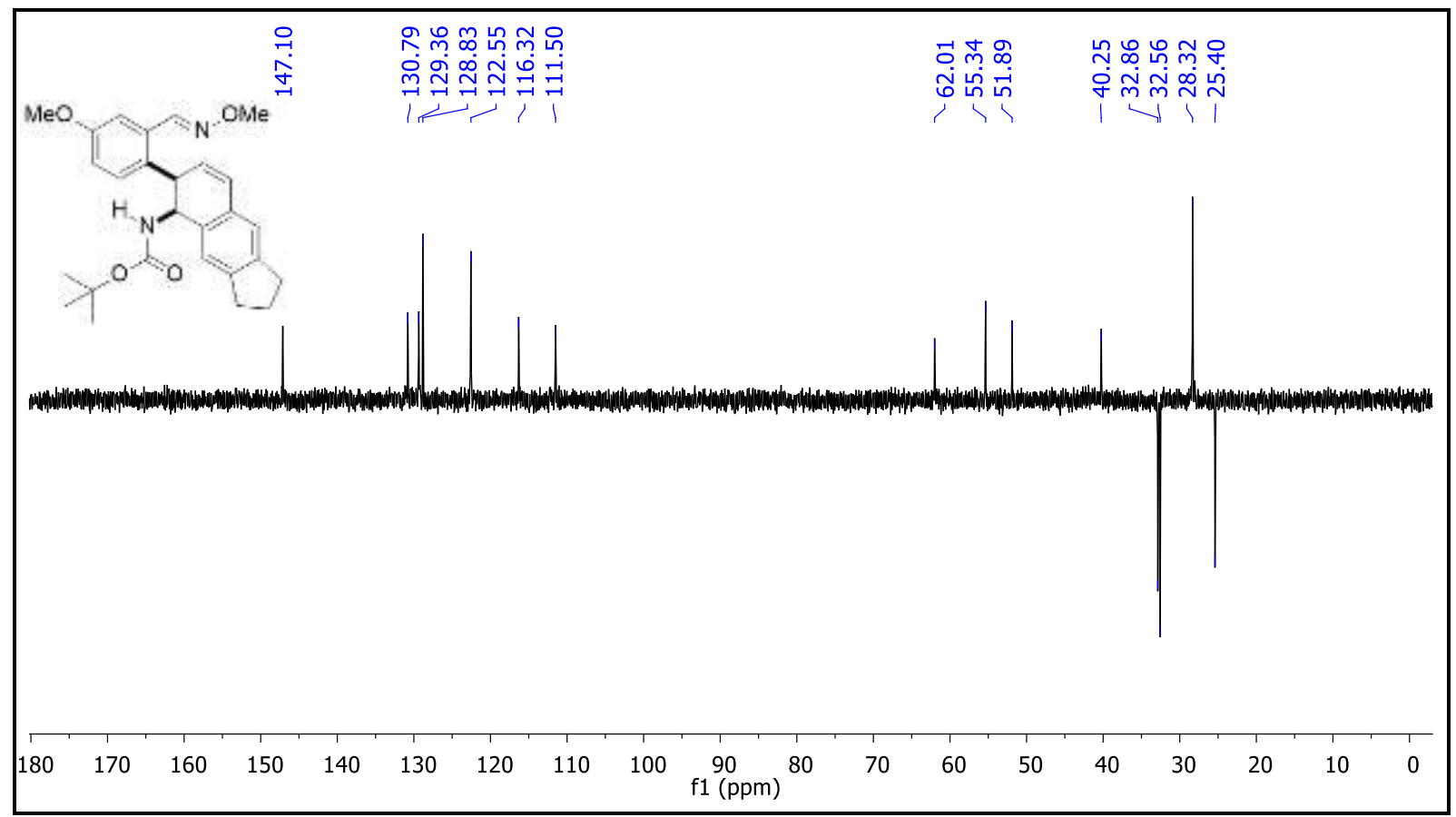


${ }^{1} \mathrm{H}$ and ${ }^{13} \mathrm{C}\left\{{ }^{1} \mathrm{H}\right\}$ NMR Spectra of Compound 3ad $\left(\mathrm{CDCl}_{3}, 400 \mathrm{MHz}\right)$.
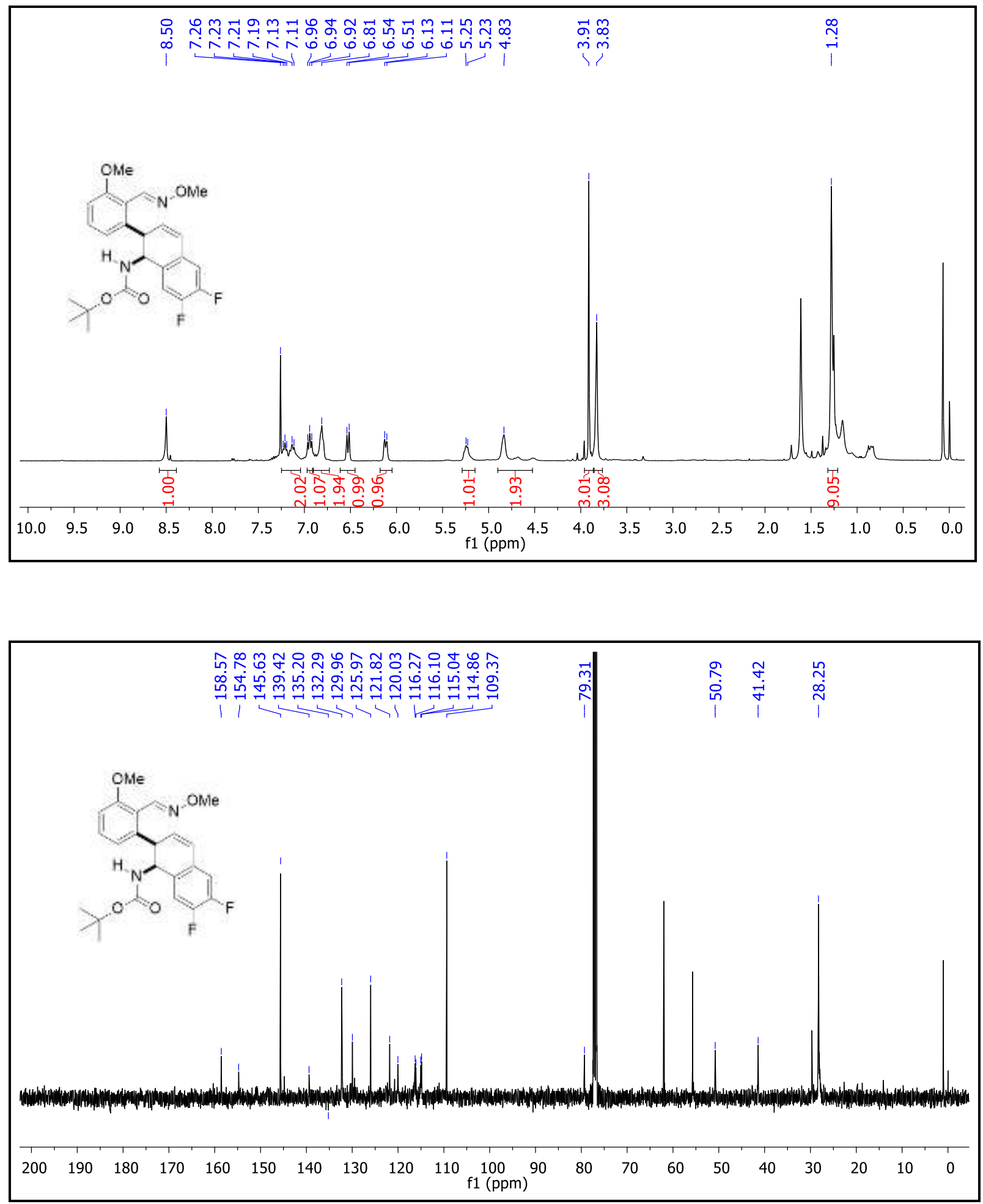
DEPT (135) NMR Spectrum of Compound 3ad $\left(\mathrm{CDCl}_{3}, 101 \mathrm{MHz}\right)$.

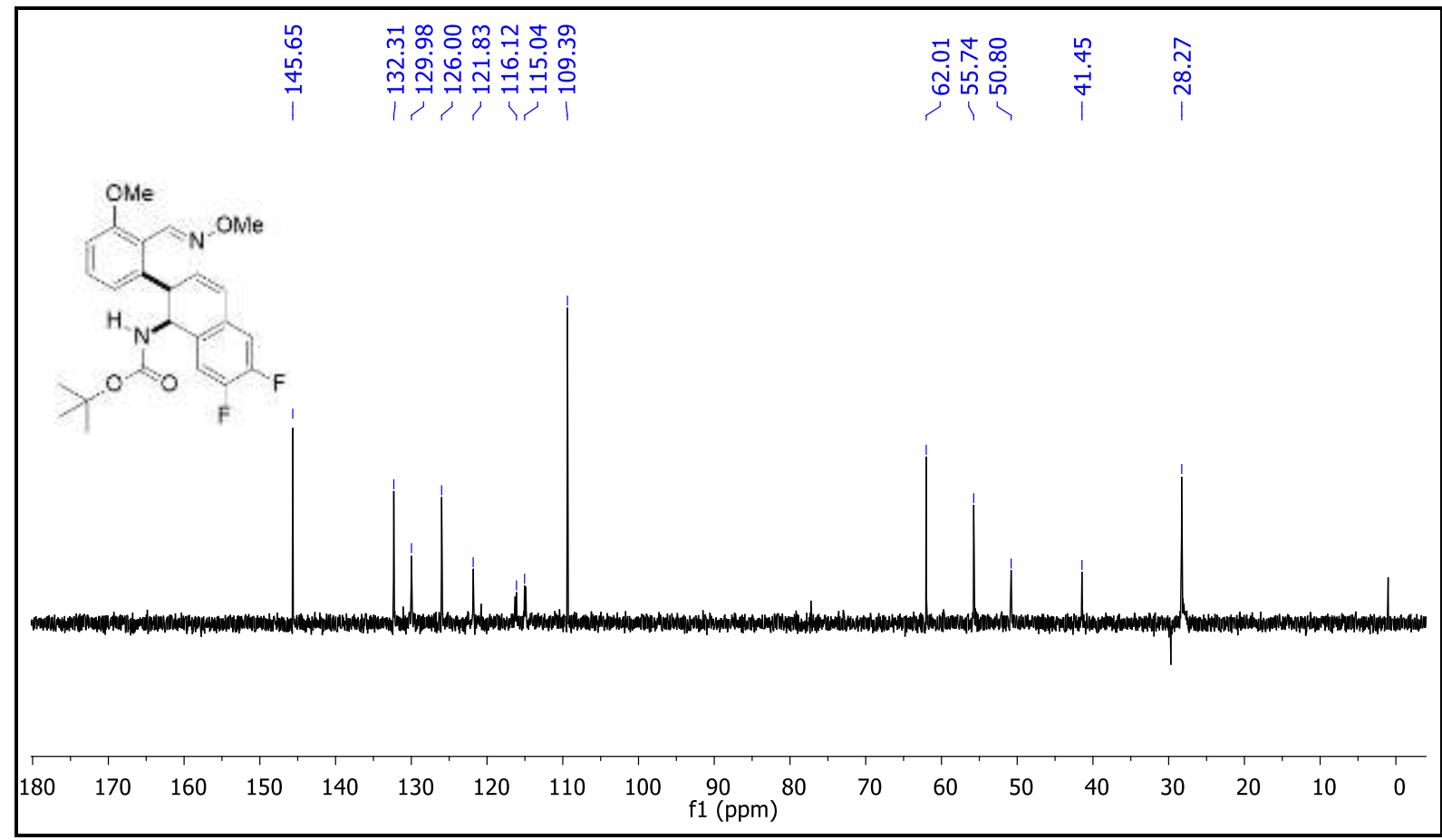

${ }^{19} \mathrm{~F}$ NMR Spectrum of Compound 3ad $\left(\mathrm{CDCl}_{3}, 471 \mathrm{MHz}\right)$.

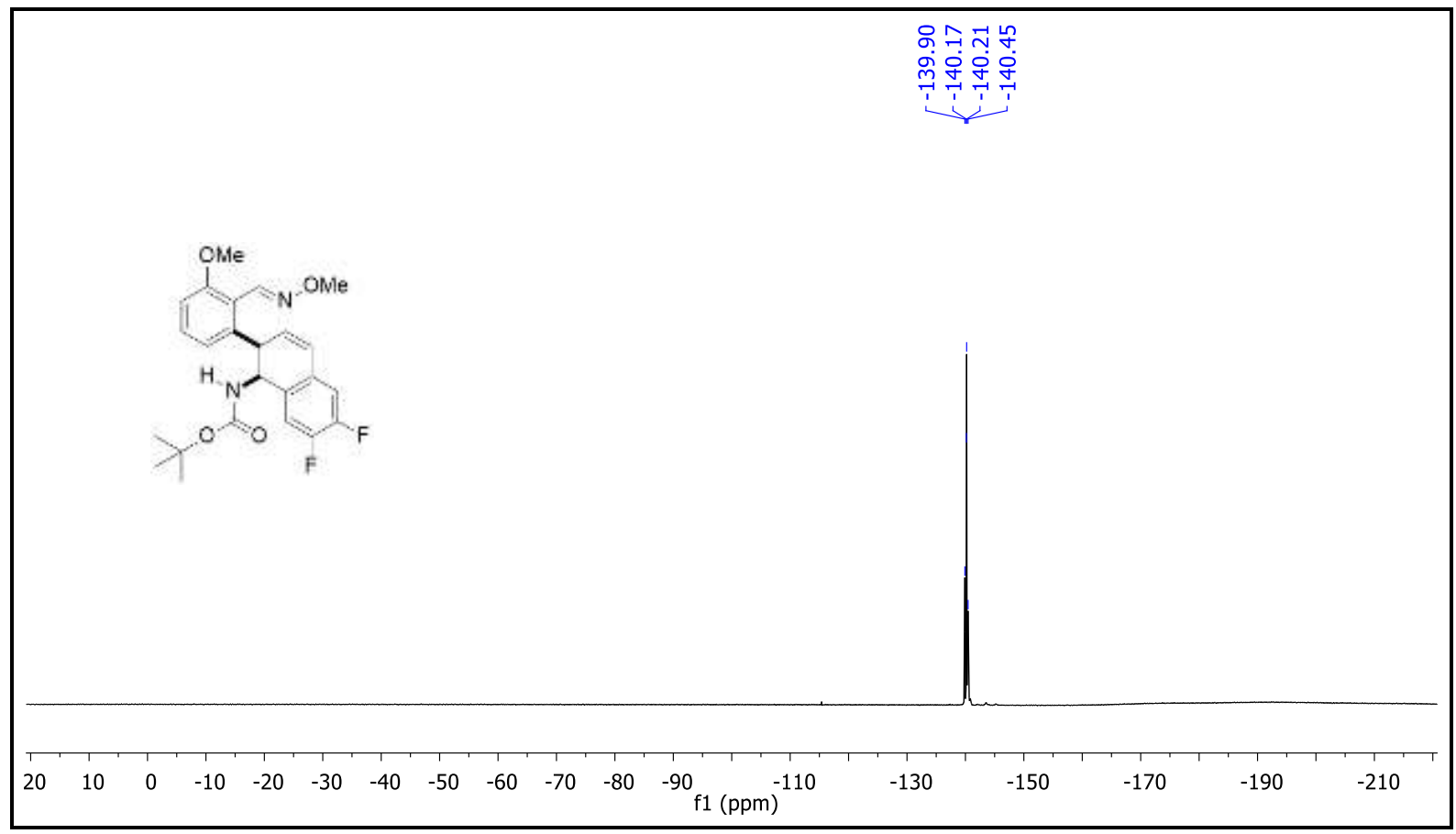


${ }^{1} \mathrm{H}$ and ${ }^{13} \mathrm{C}\left\{{ }^{1} \mathrm{H}\right\}$ NMR Spectra of Compound 3gd $\left(\mathrm{CDCl}_{3}, 400 \mathrm{MHz}\right)$.
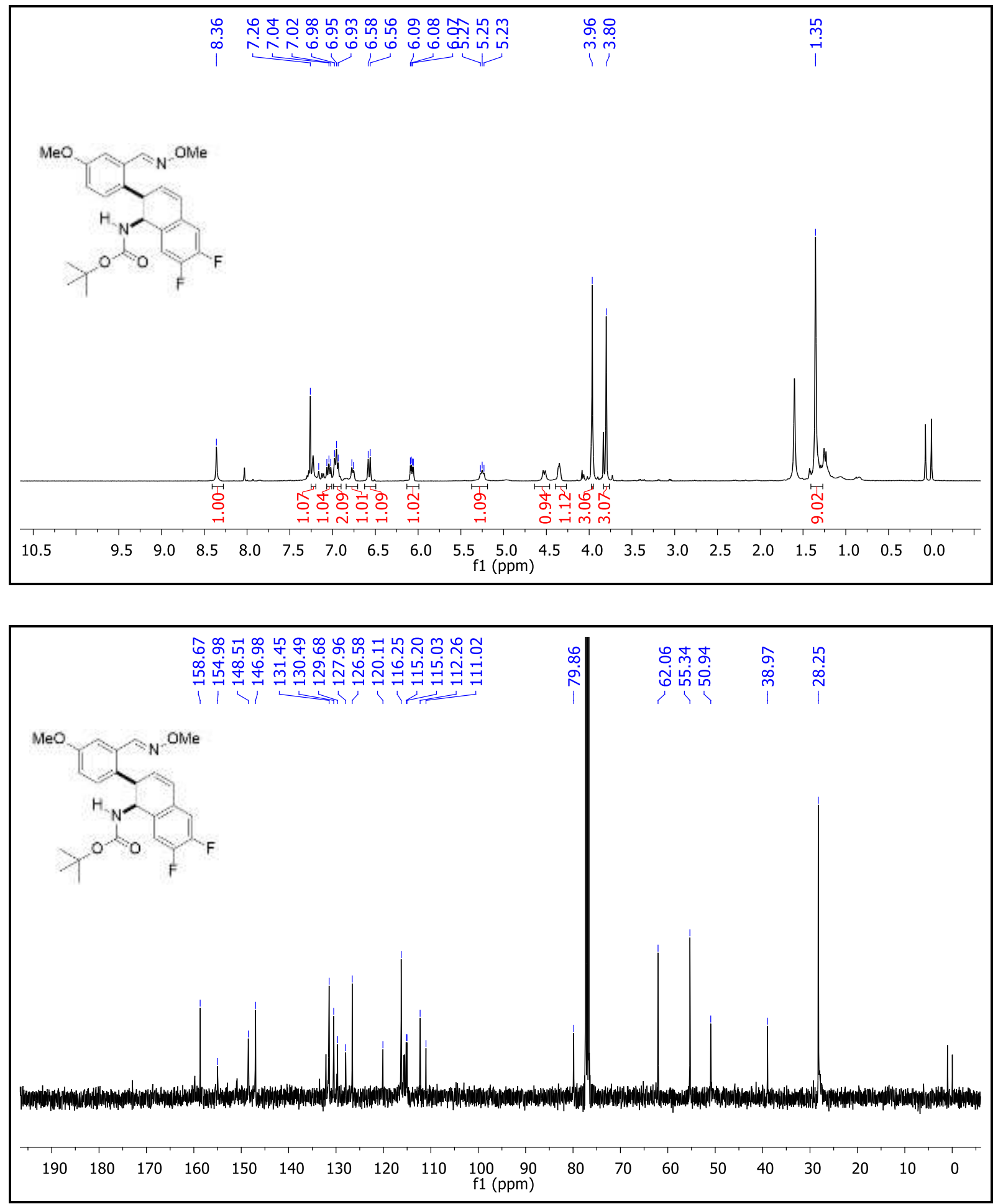
DEPT (135) NMR Spectrum of Compound 3gd $\left(\mathrm{CDCl}_{3}, 101 \mathrm{MHz}\right)$.

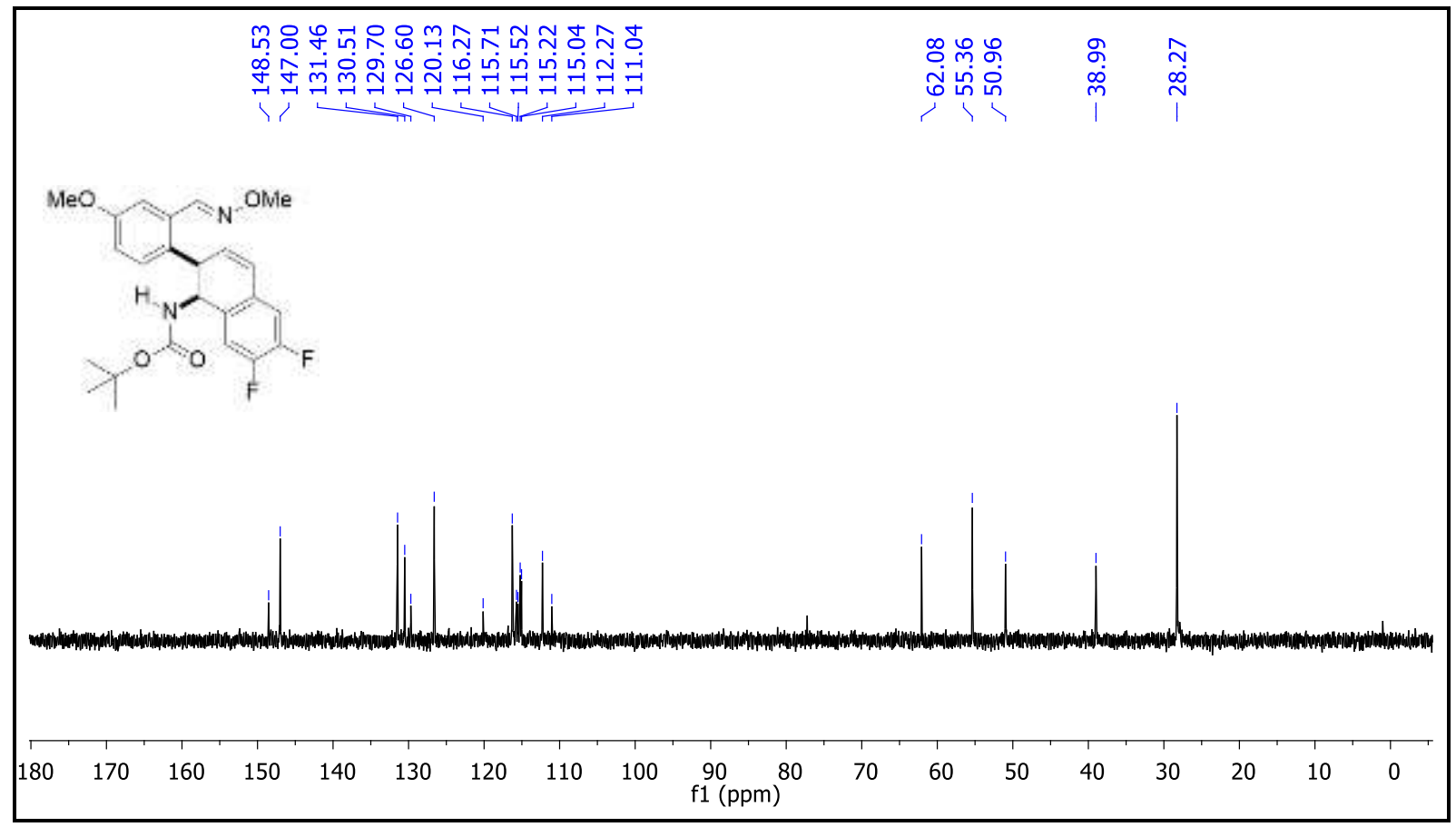

${ }^{19} \mathrm{~F}$ NMR Spectrum of Compound 3gd $\left(\mathrm{CDCl}_{3}, 471 \mathrm{MHz}\right)$.

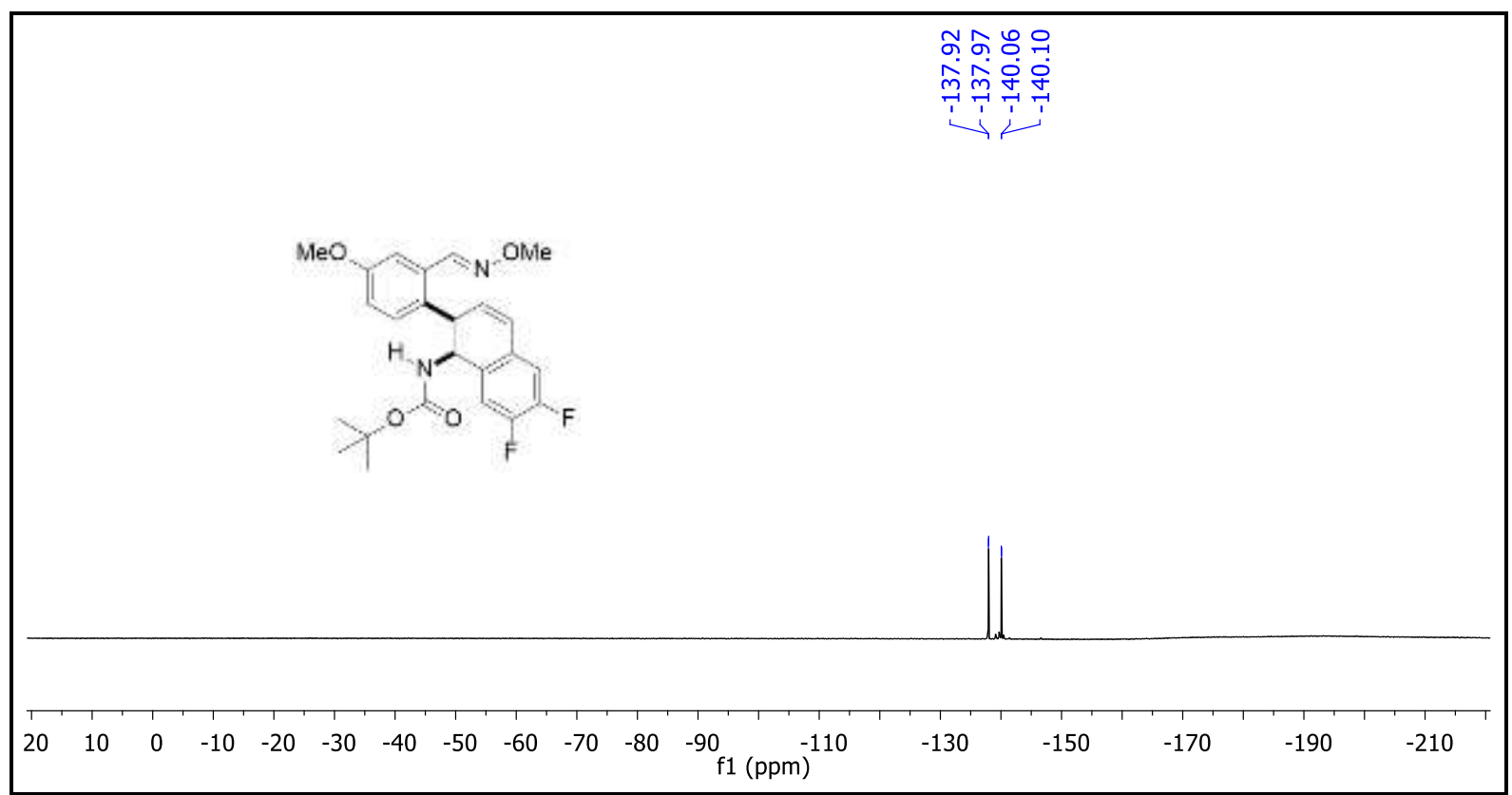


${ }^{1} \mathrm{H}$ and ${ }^{13} \mathrm{C}\left\{{ }^{1} \mathrm{H}\right\}$ NMR Spectra of Compound 3ae $\left(\mathrm{CDCl}_{3}, 400 \mathrm{MHz}\right)$.
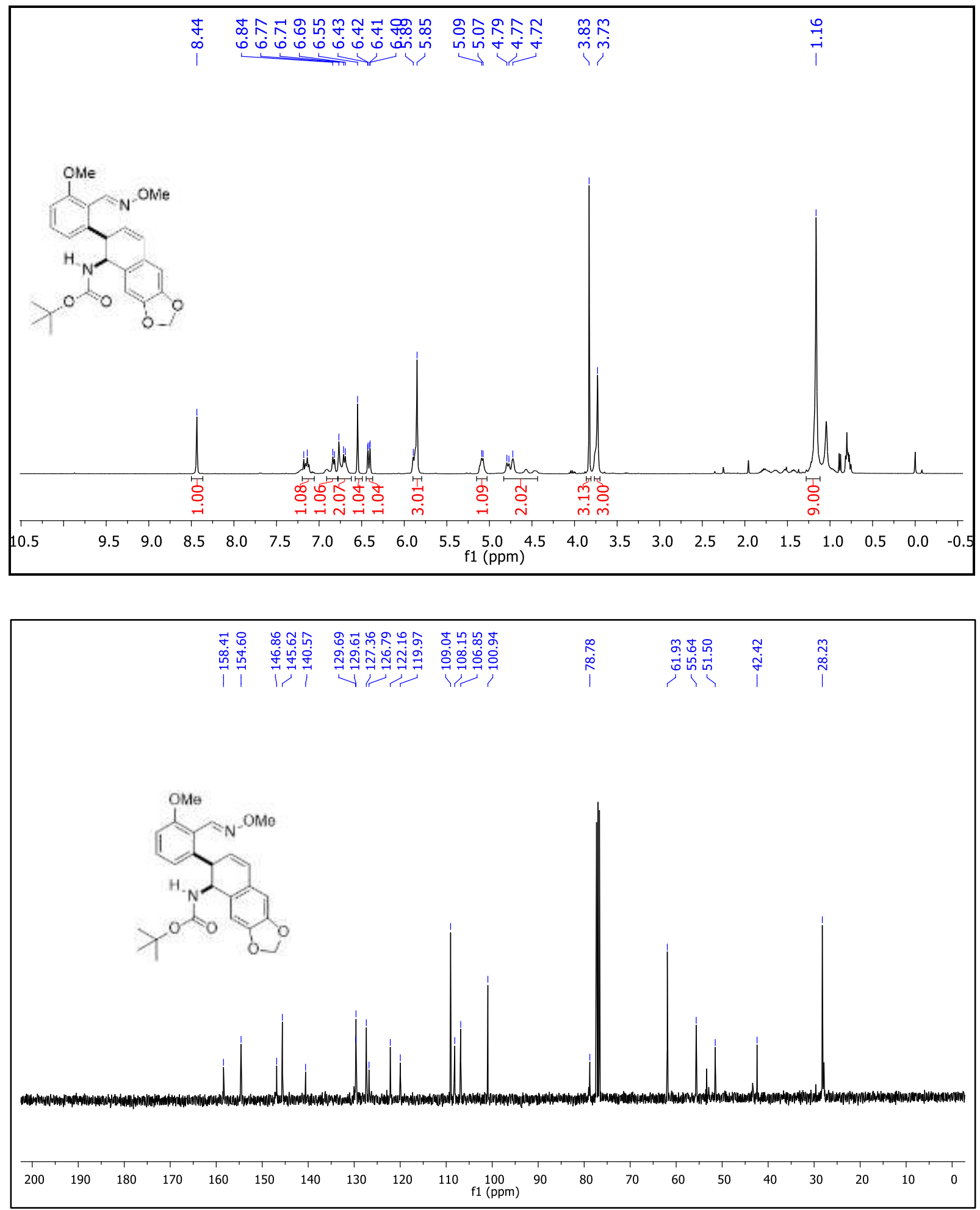
DEPT (135) NMR Spectrum of Compound 3ae $\left(\mathrm{CDCl}_{3}, 101 \mathrm{MHz}\right)$.

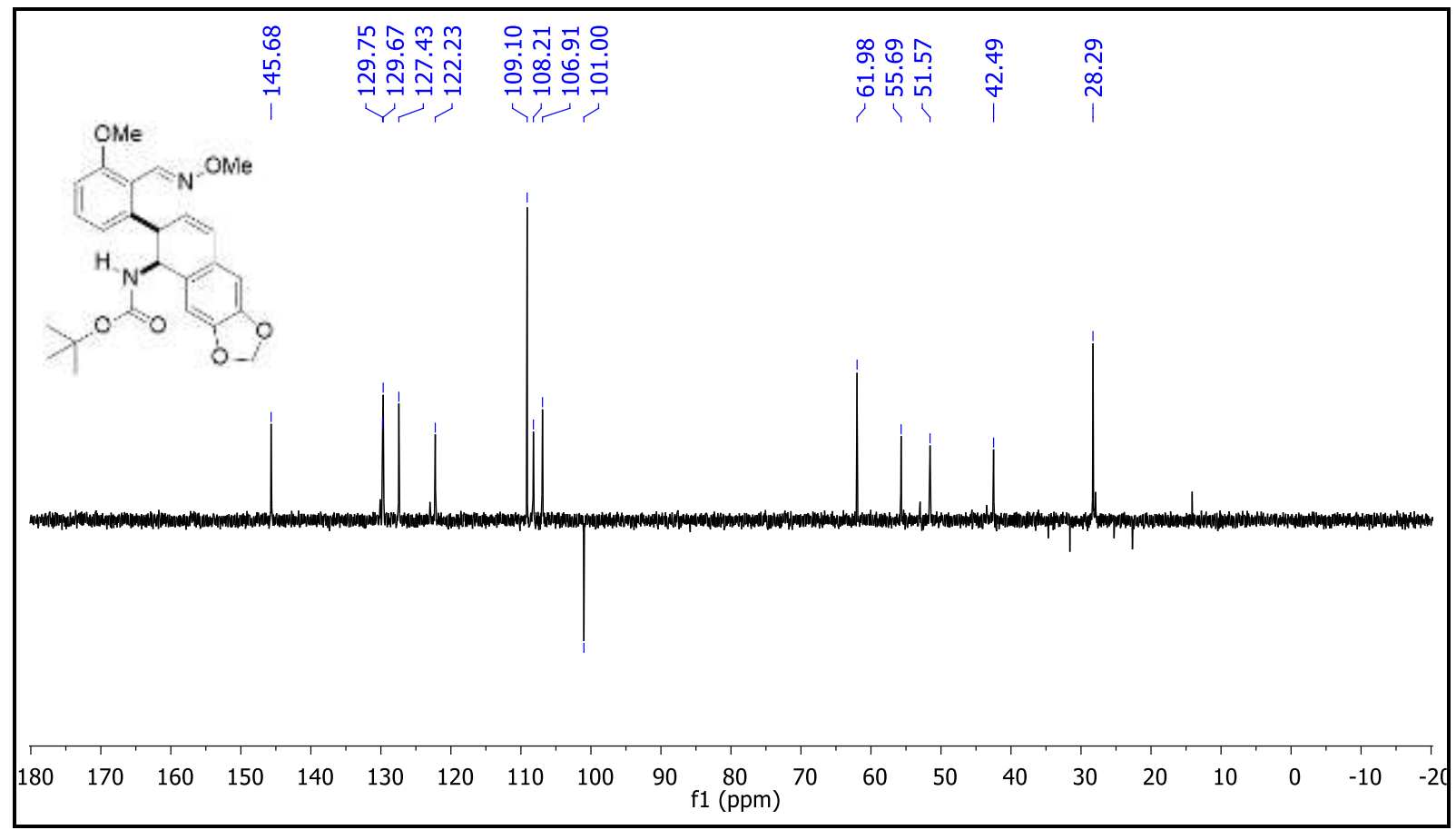


${ }^{1} \mathrm{H}$ and ${ }^{13} \mathrm{C}\left\{{ }^{1} \mathrm{H}\right\}$ NMR Spectra of Compound 3be $\left(\mathrm{CDCl}_{3}, 400 \mathrm{MHz}\right)$.
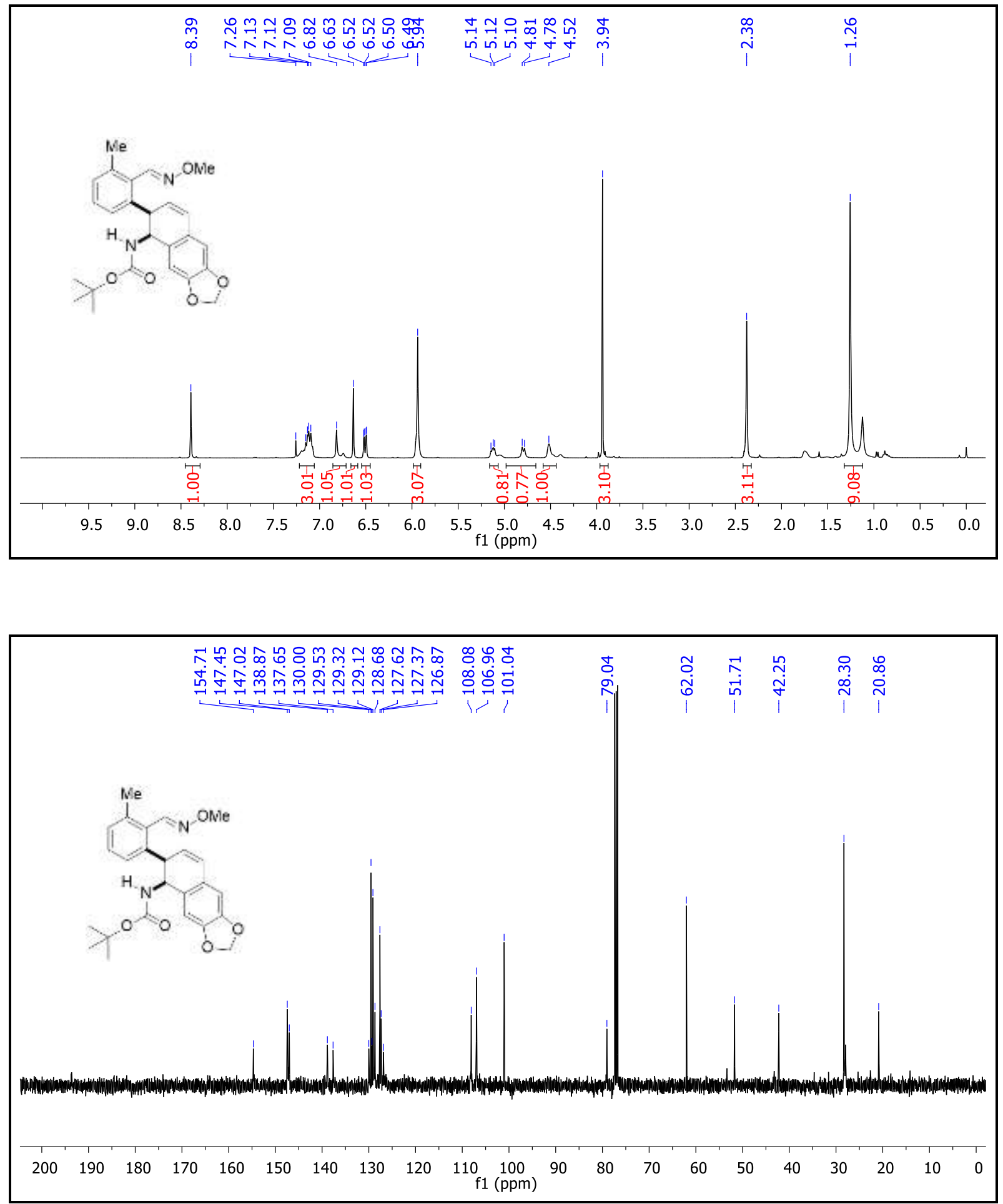
DEPT (135) NMR Spectrum of Compound 3be $\left(\mathrm{CDCl}_{3}, 101 \mathrm{MHz}\right)$.

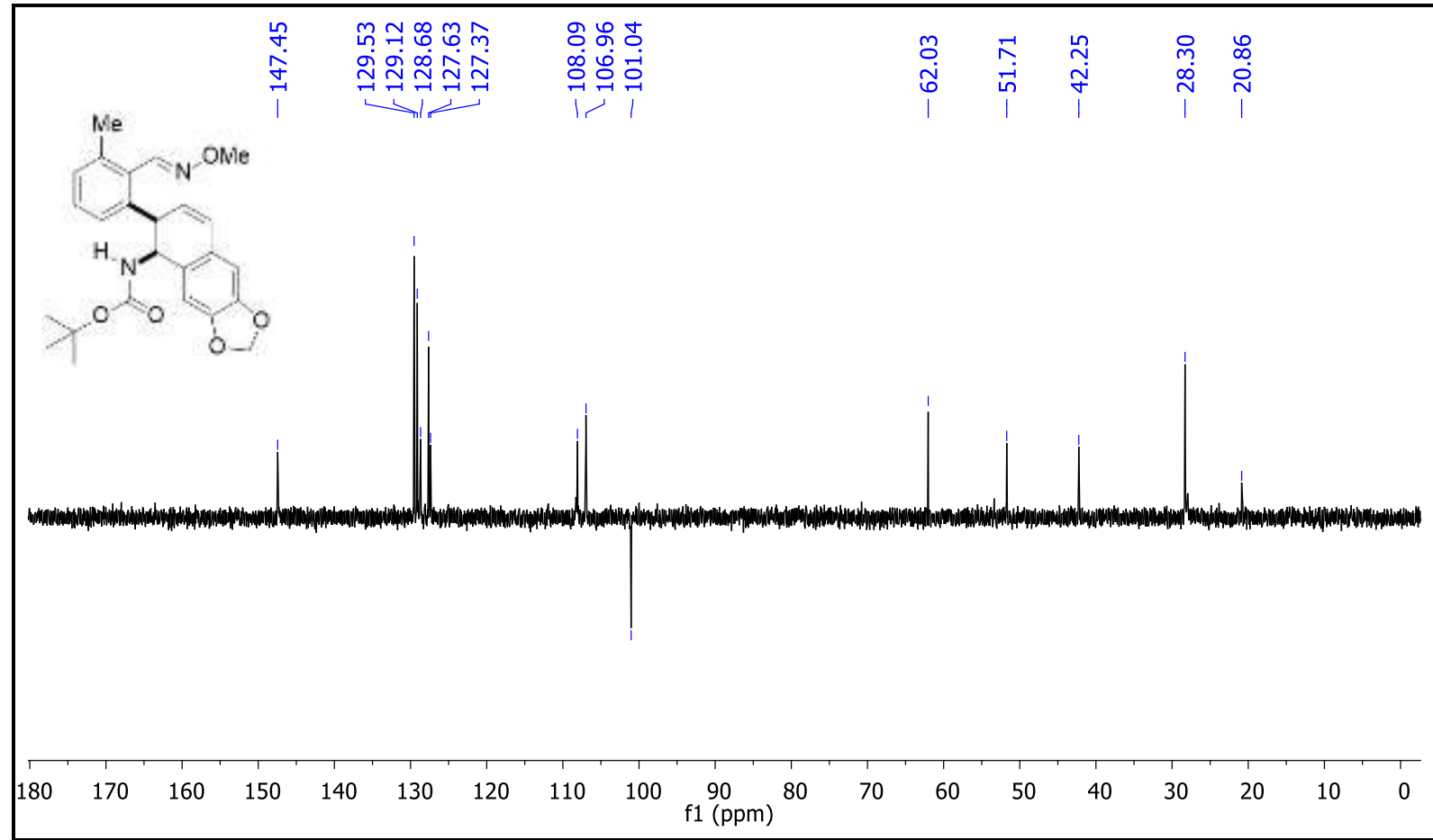


${ }^{1} \mathrm{H}$ and ${ }^{13} \mathrm{C}\left\{{ }^{1} \mathrm{H}\right\}$ NMR Spectra of Compound $3 f e\left(\mathrm{CDCl}_{3}, 400 \mathrm{MHz}\right)$.
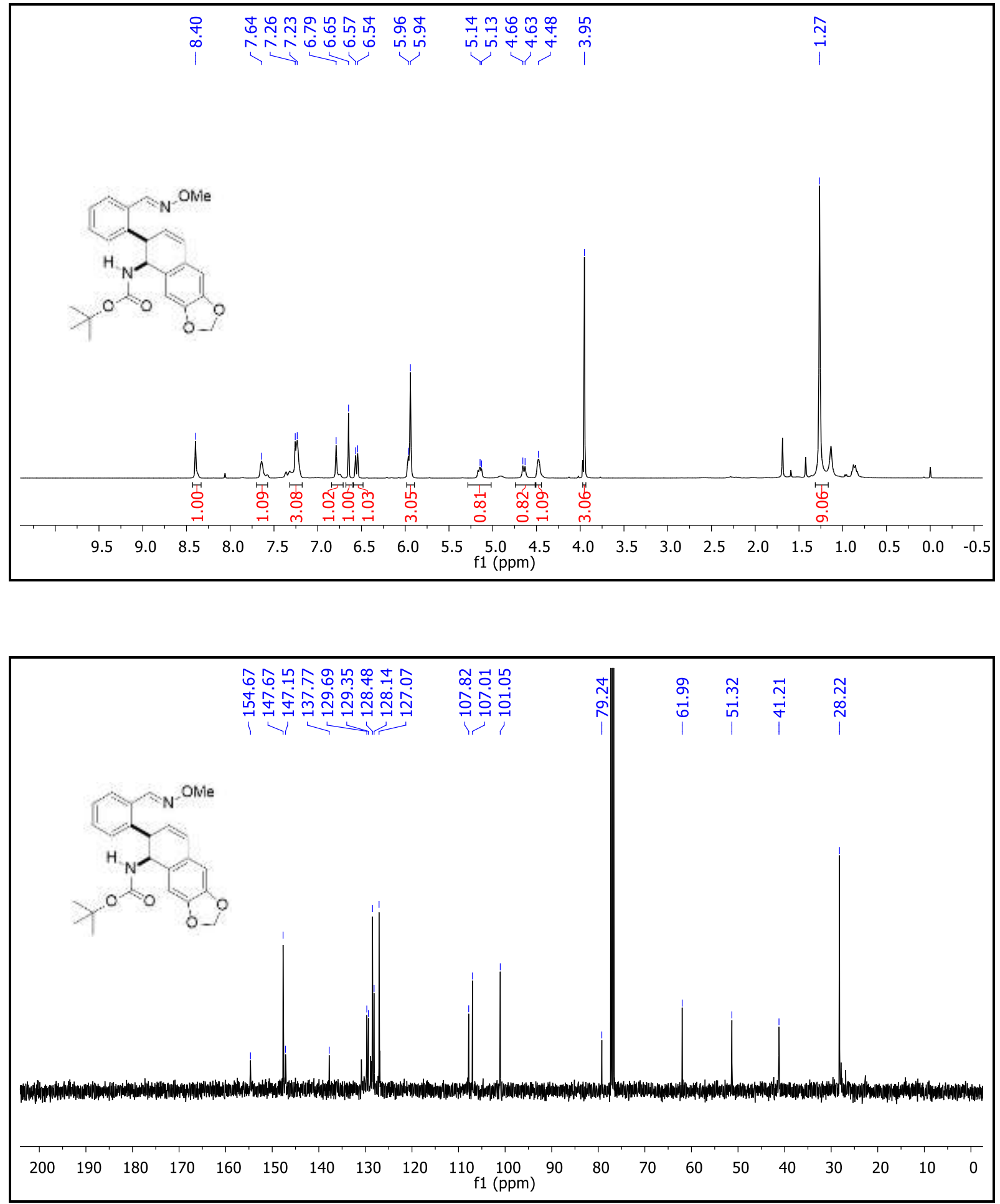
DEPT (135) NMR Spectrum of Compound $\mathbf{3 f e}\left(\mathrm{CDCl}_{3}, 101 \mathrm{MHz}\right)$.

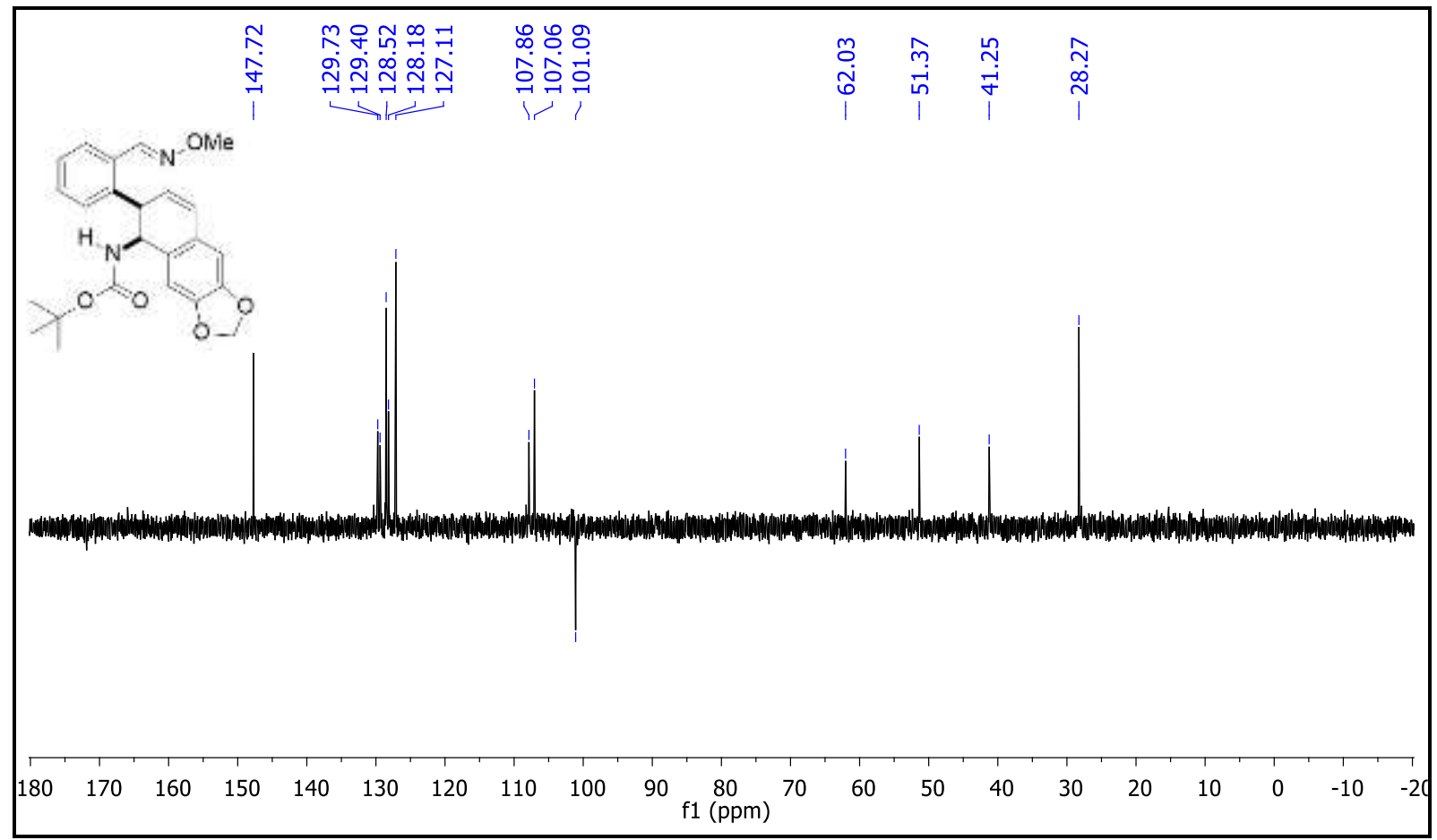


${ }^{1} \mathrm{H}$ and ${ }^{13} \mathrm{C}\left\{{ }^{1} \mathrm{H}\right\}$ NMR Spectra of Compound 3af $\left(\mathrm{CDCl}_{3}, 400 \mathrm{MHz}\right)$.
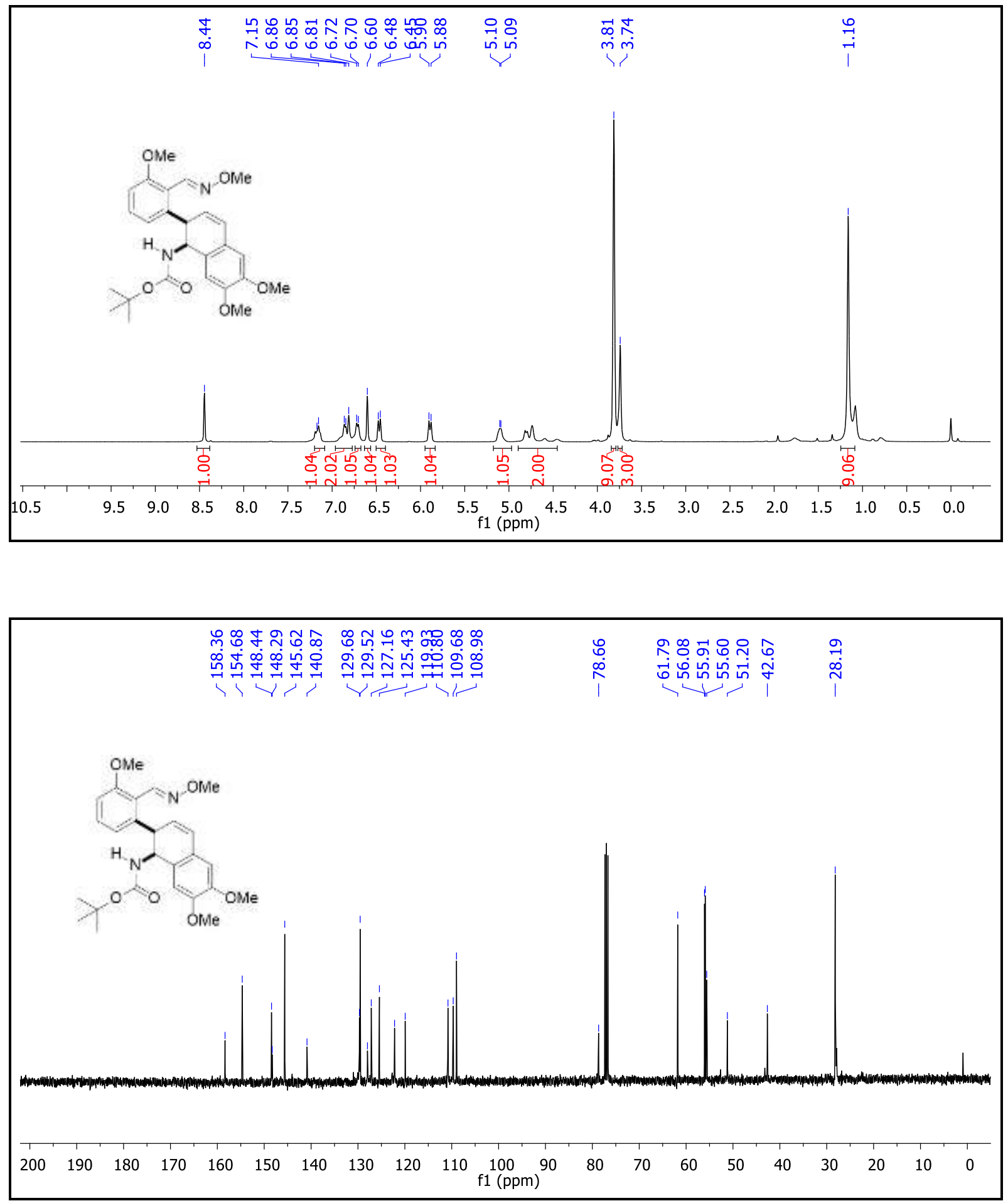
DEPT (135) NMR Spectrum of Compound 3af $\left(\mathrm{CDCl}_{3}, 101 \mathrm{MHz}\right)$.

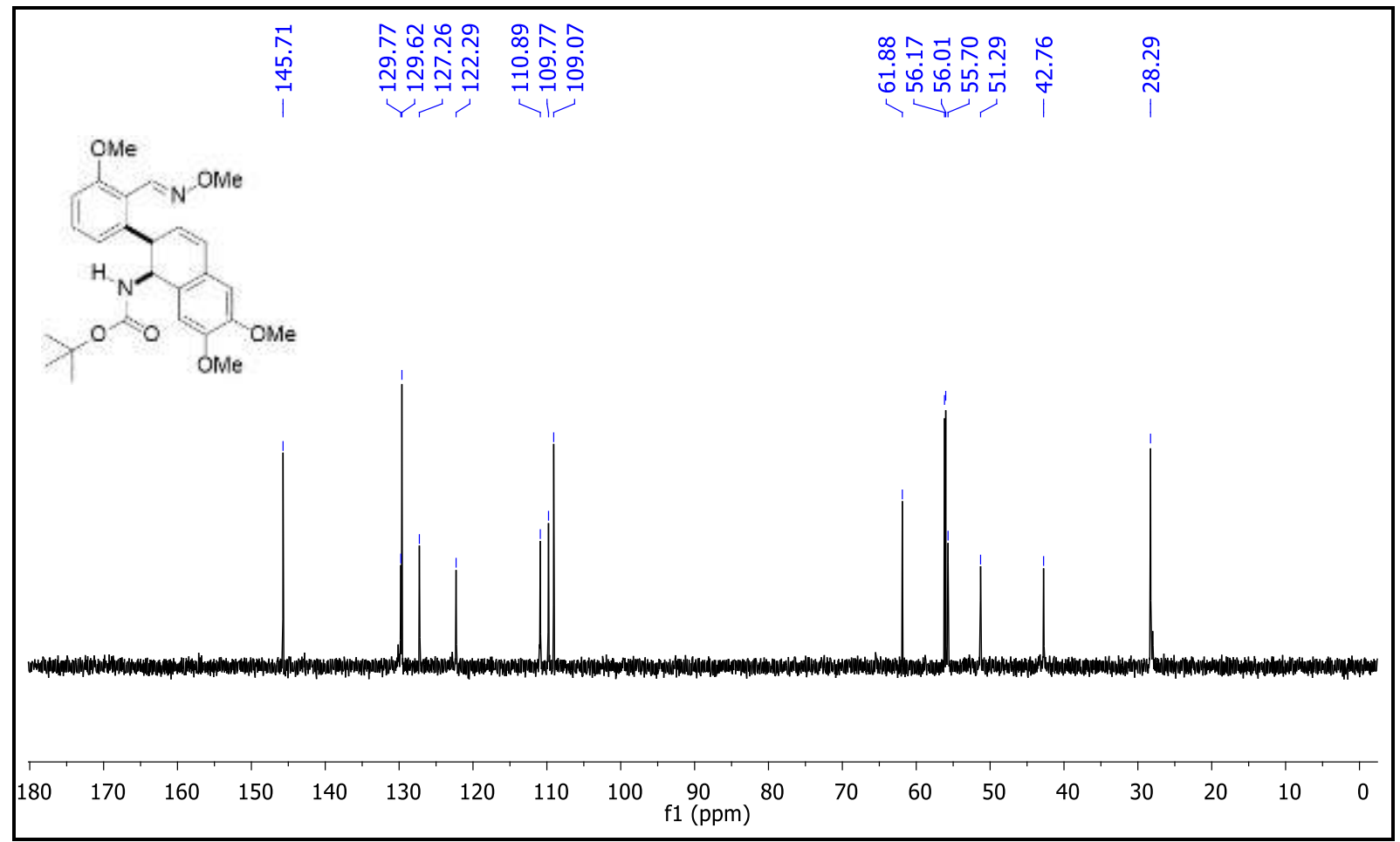


${ }^{1} \mathrm{H}$ and ${ }^{13} \mathrm{C}\left\{{ }^{1} \mathrm{H}\right\}$ NMR Spectra of Compound $3 \mathbf{b f}\left(\mathrm{CDCl}_{3}, 400 \mathrm{MHz}\right)$.
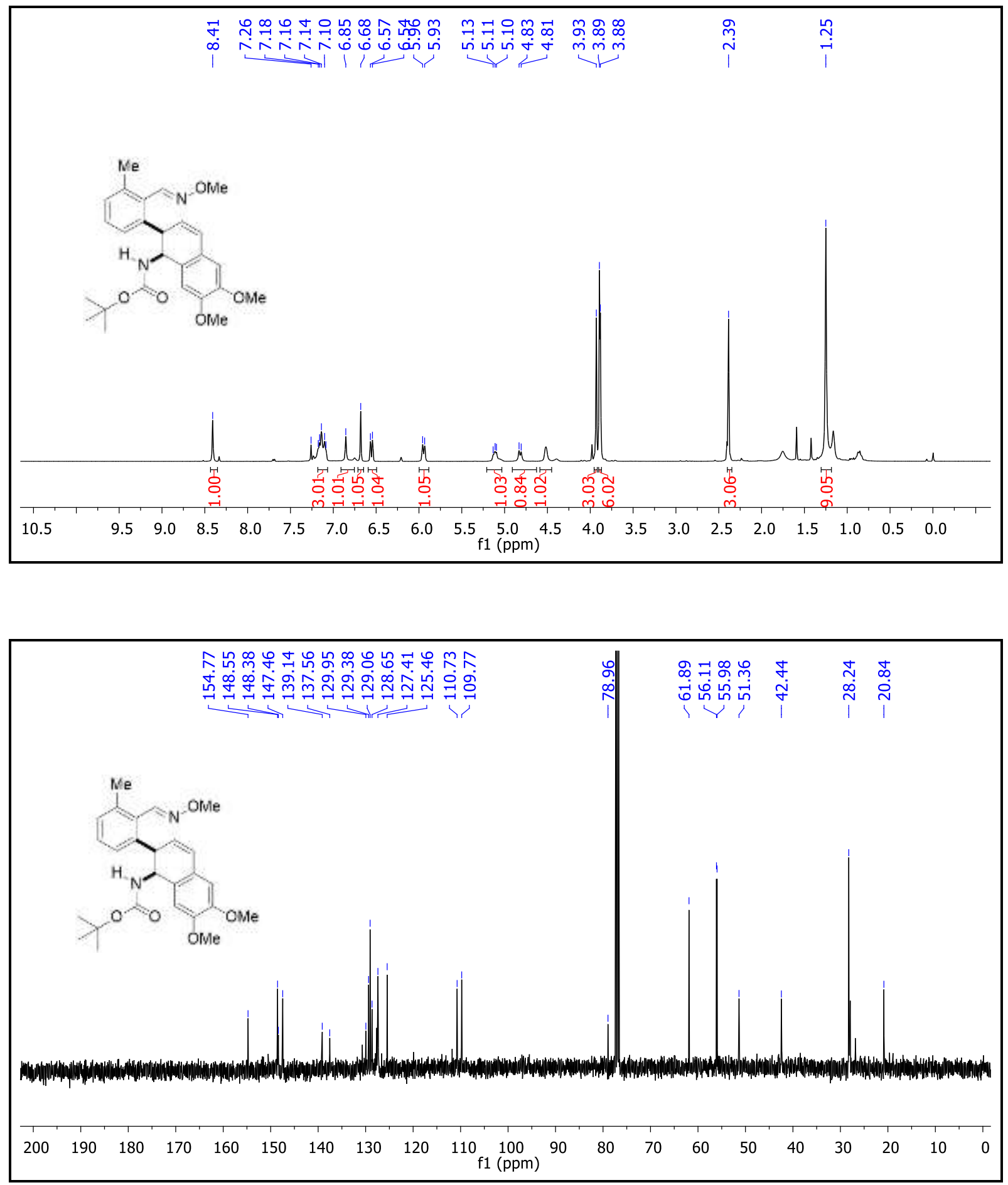
DEPT (135) NMR Spectrum of Compound $\mathbf{3 b f}\left(\mathrm{CDCl}_{3}, 101 \mathrm{MHz}\right)$.

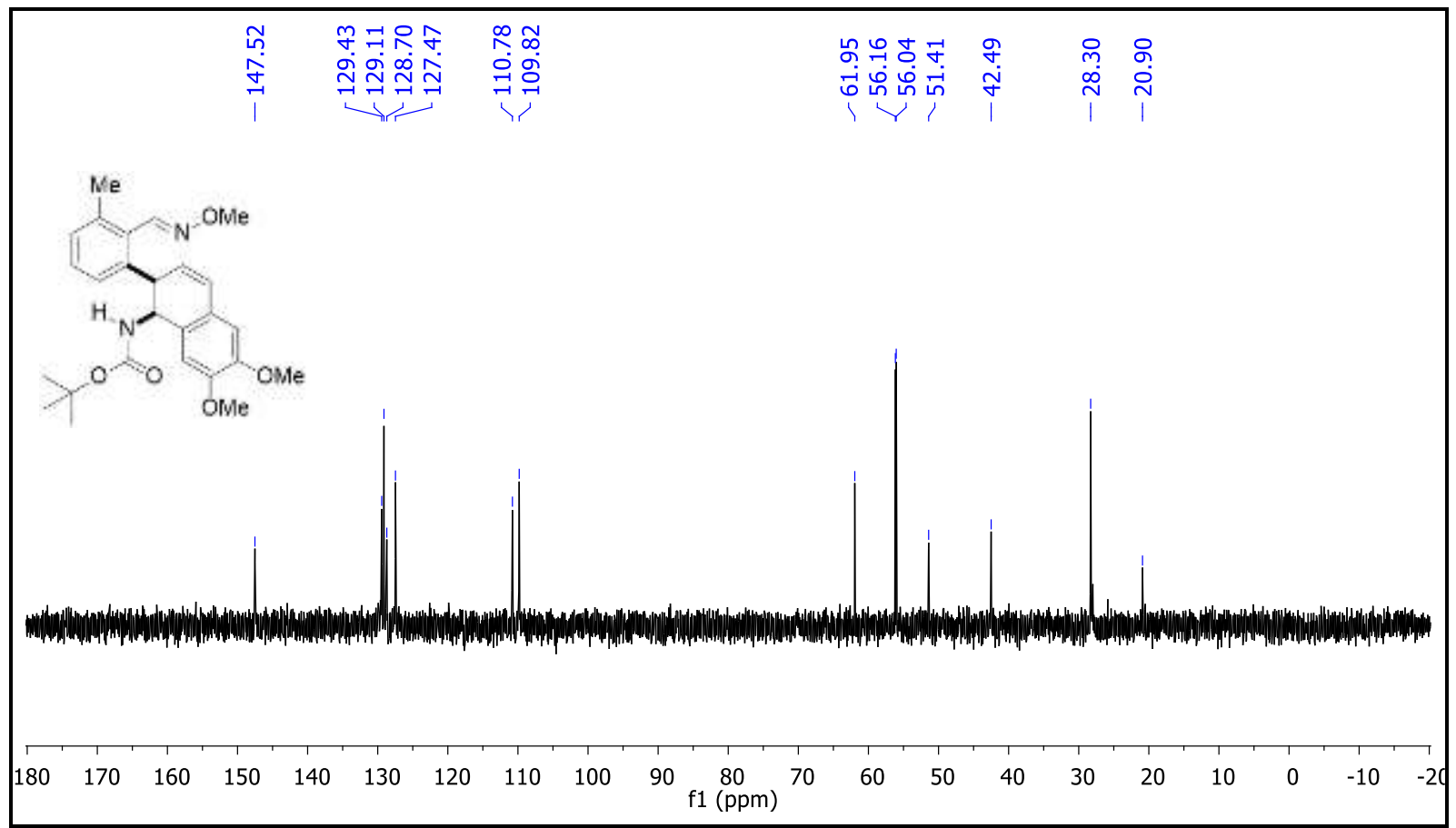


${ }^{1} \mathrm{H}$ and ${ }^{13} \mathrm{C}\left\{{ }^{1} \mathrm{H}\right\}$ NMR Spectra of Compound $3 f f\left(\mathrm{CDCl}_{3}, 400 \mathrm{MHz}\right)$.
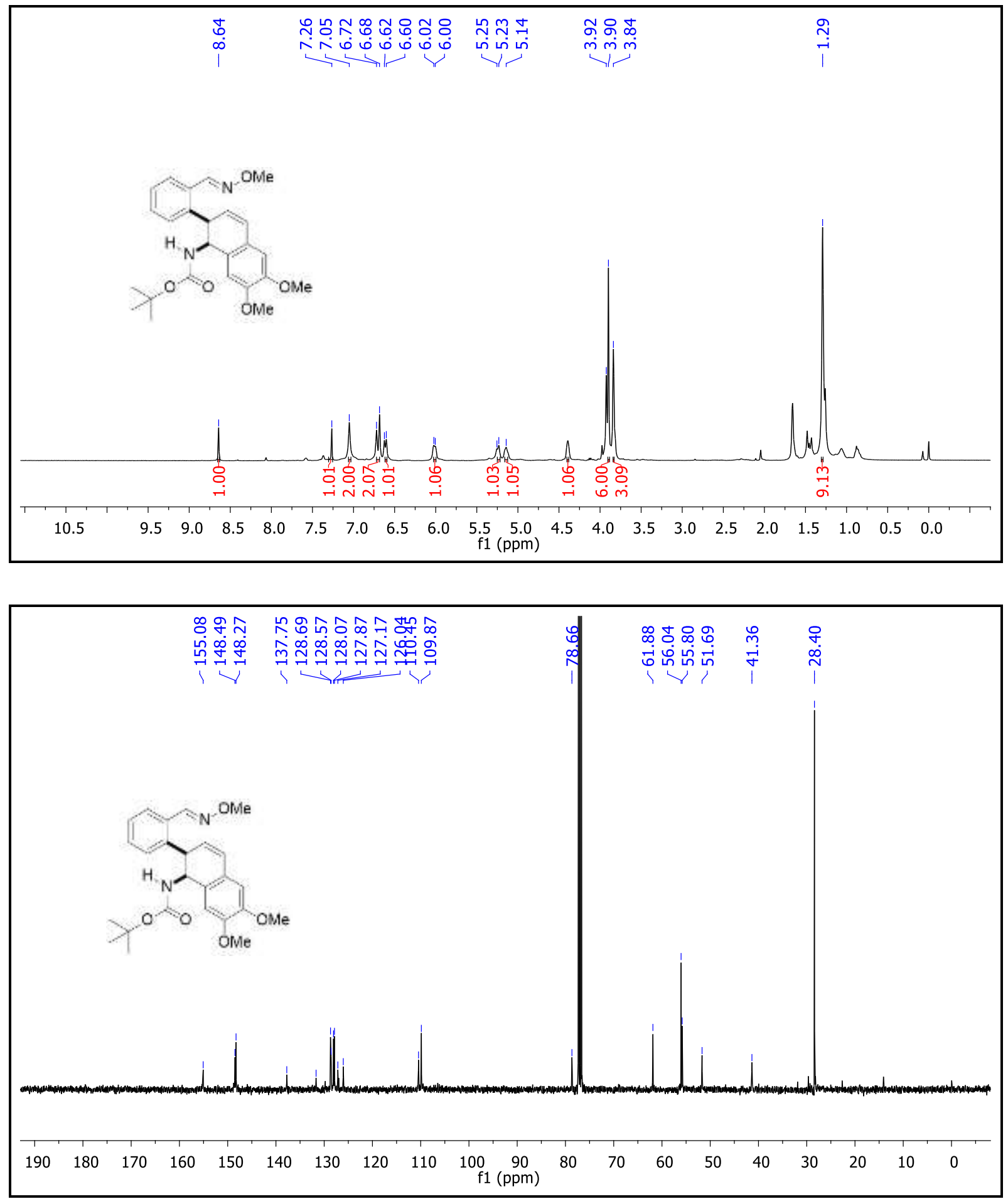
DEPT (135) NMR Spectrum of Compound $\mathbf{3 f f}\left(\mathrm{CDCl}_{3}, 101 \mathrm{MHz}\right)$.

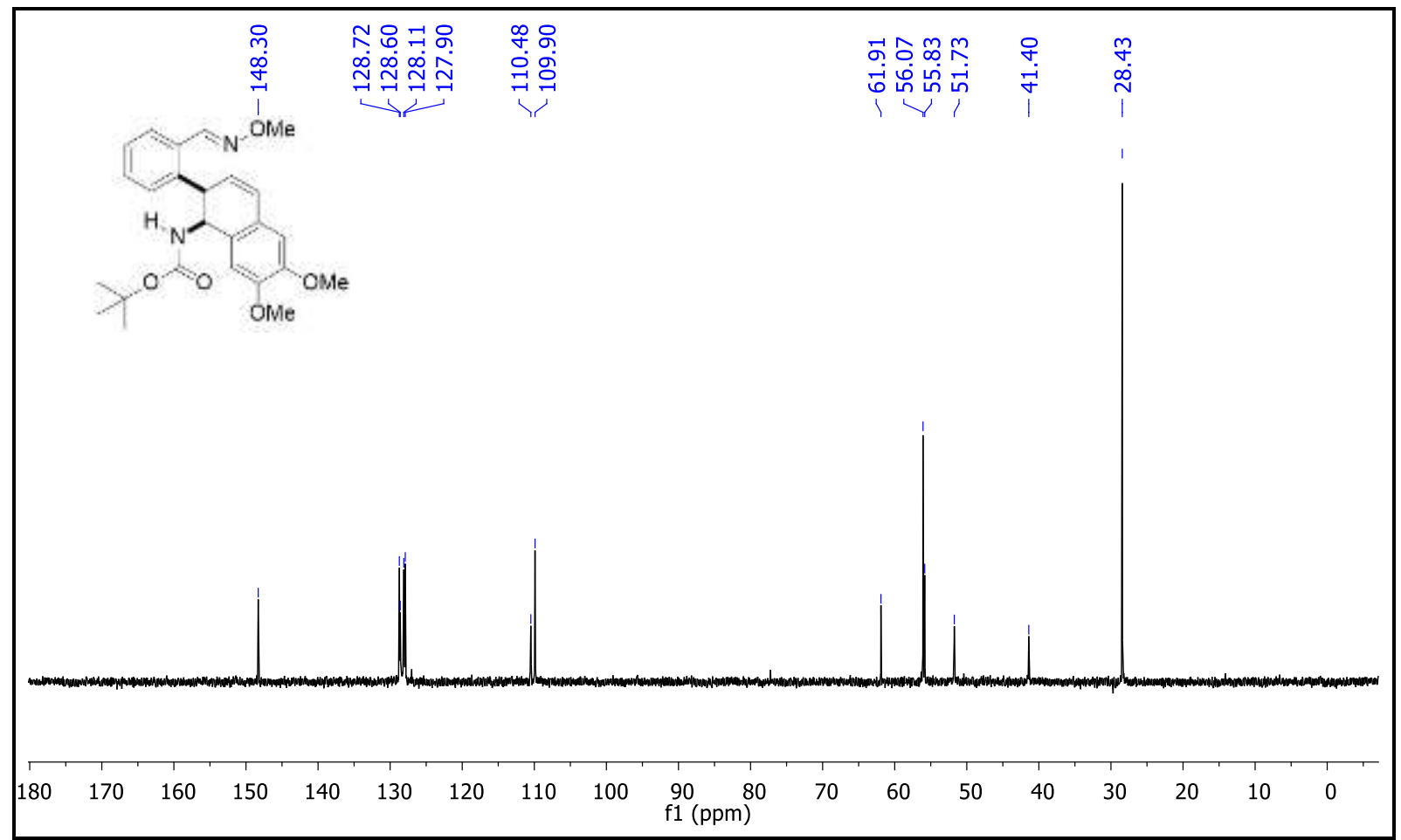


${ }^{1} \mathrm{H}$ and ${ }^{13} \mathrm{C}\left\{{ }^{1} \mathrm{H}\right\}$ NMR Spectra of Compound 3ke $\left(\mathrm{CDCl}_{3}, 400 \mathrm{MHz}\right)$.
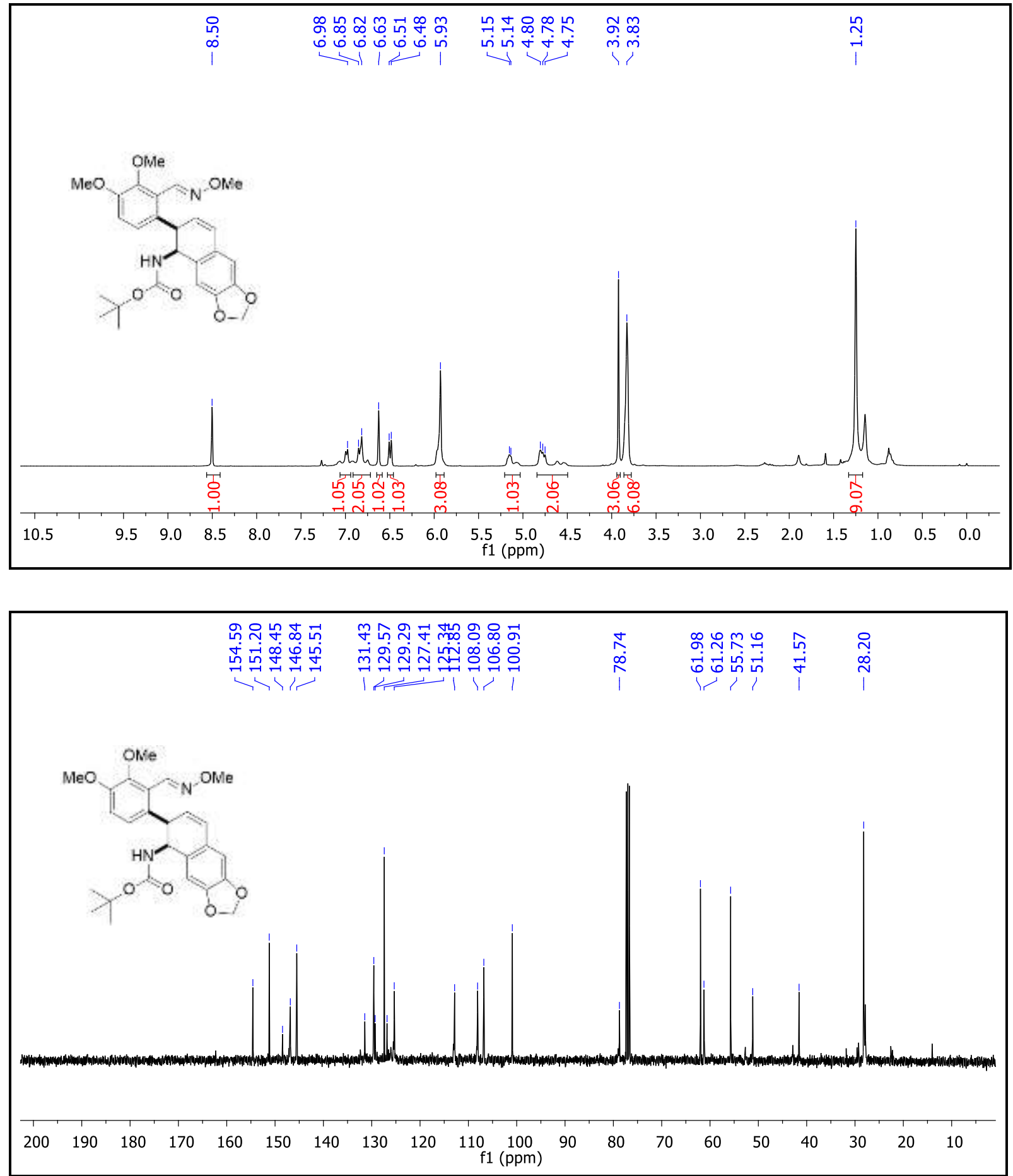
DEPT (135) NMR Spectrum of Compound 3ke $\left(\mathrm{CDCl}_{3}, 101 \mathrm{MHz}\right)$.

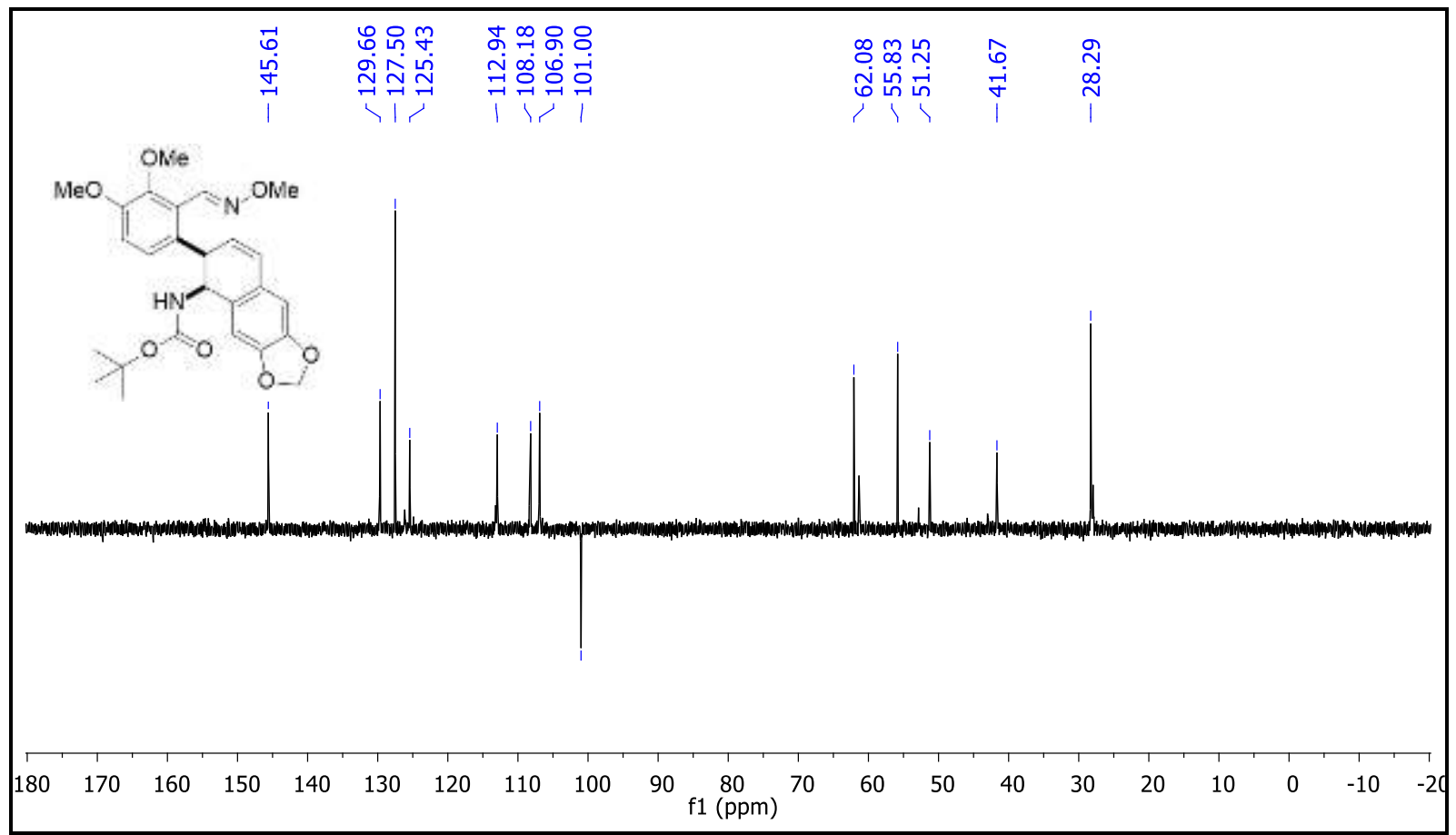


${ }^{1} \mathrm{H}$ and ${ }^{13} \mathrm{C}\left\{{ }^{1} \mathrm{H}\right\}$ NMR Spectra of Compound 3me $\left(\mathrm{CDCl}_{3}, 400 \mathrm{MHz}\right)$.
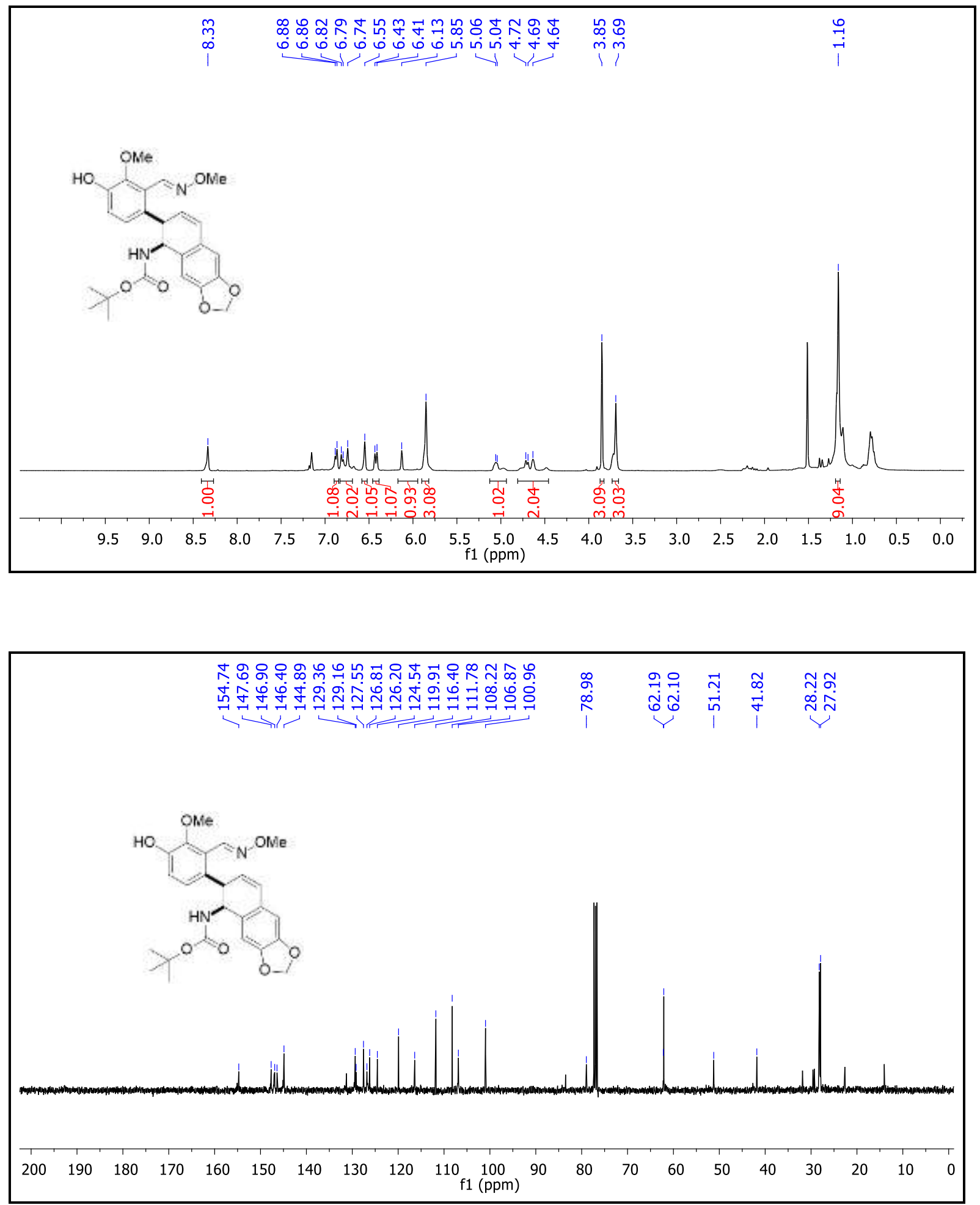
DEPT (135) NMR Spectrum of Compound 3me ( $\left.\mathrm{CDCl}_{3}, 101 \mathrm{MHz}\right)$.

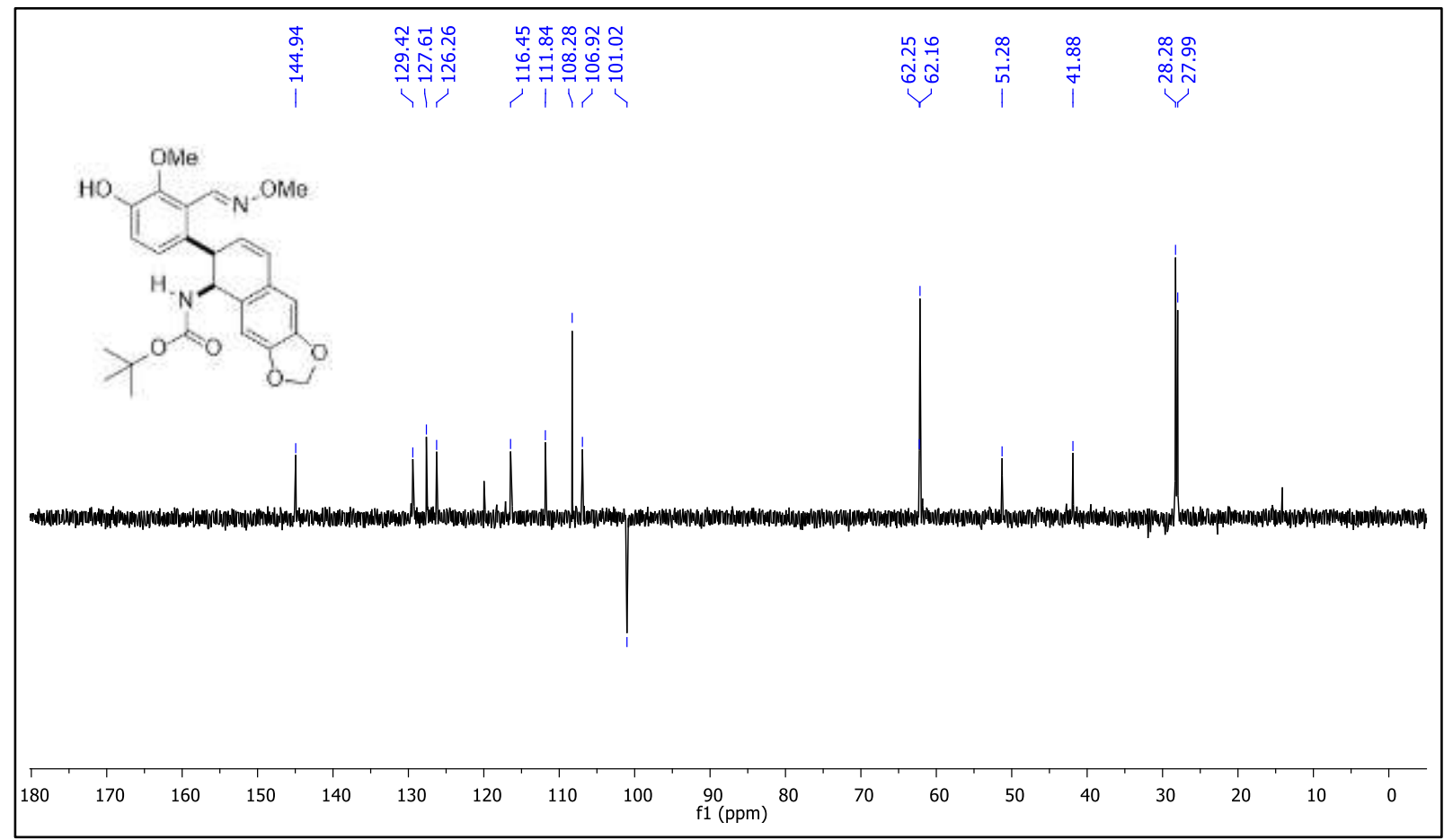


${ }^{1} \mathrm{H}$ and ${ }^{13} \mathrm{C}\left\{{ }^{1} \mathrm{H}\right\}$ NMR Spectra of Compound 3ne $\left(\mathrm{CDCl}_{3}, 400 \mathrm{MHz}\right)$.
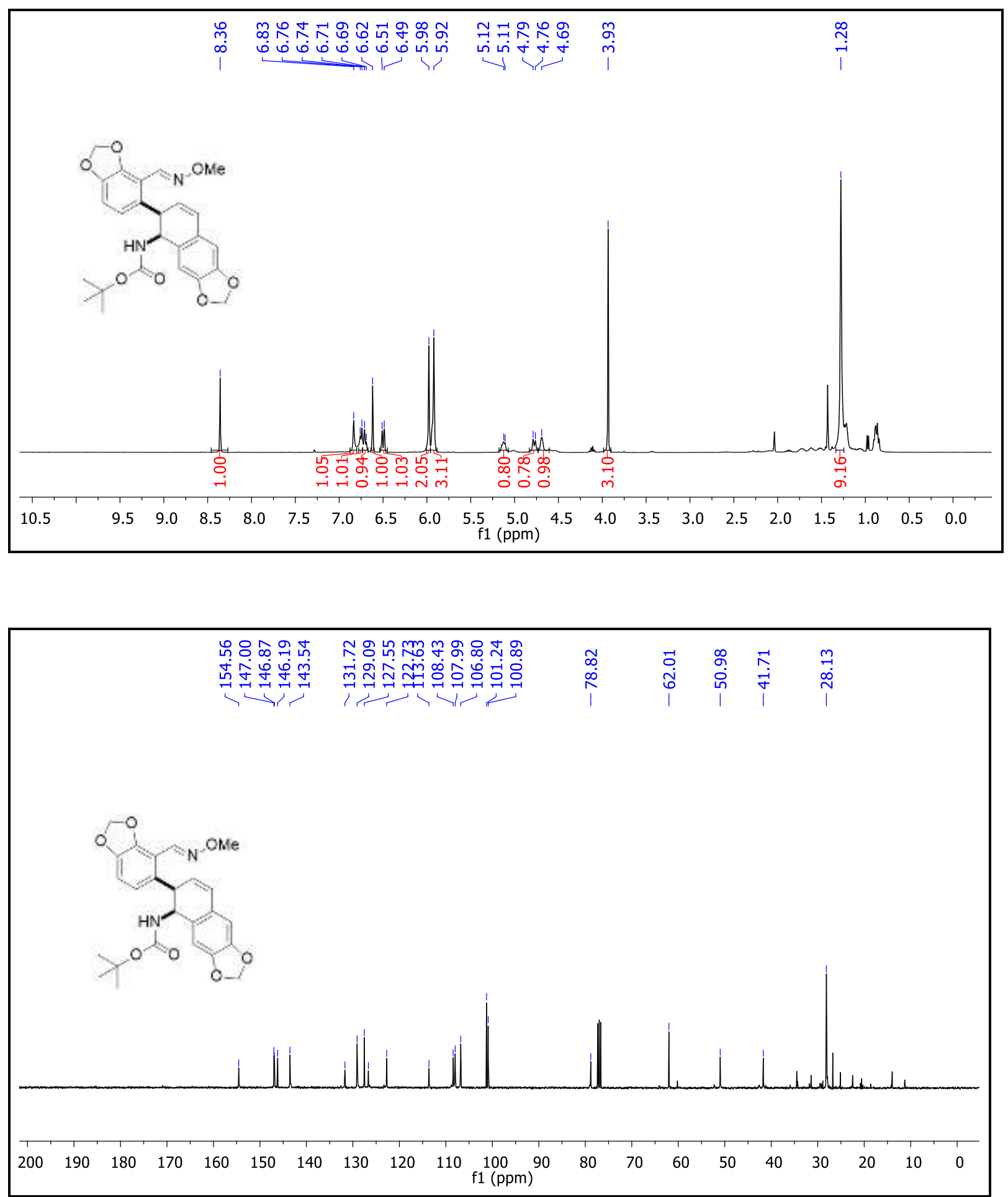
DEPT (135) NMR Spectrum of Compound 3ne $\left(\mathrm{CDCl}_{3}, 101 \mathrm{MHz}\right)$.

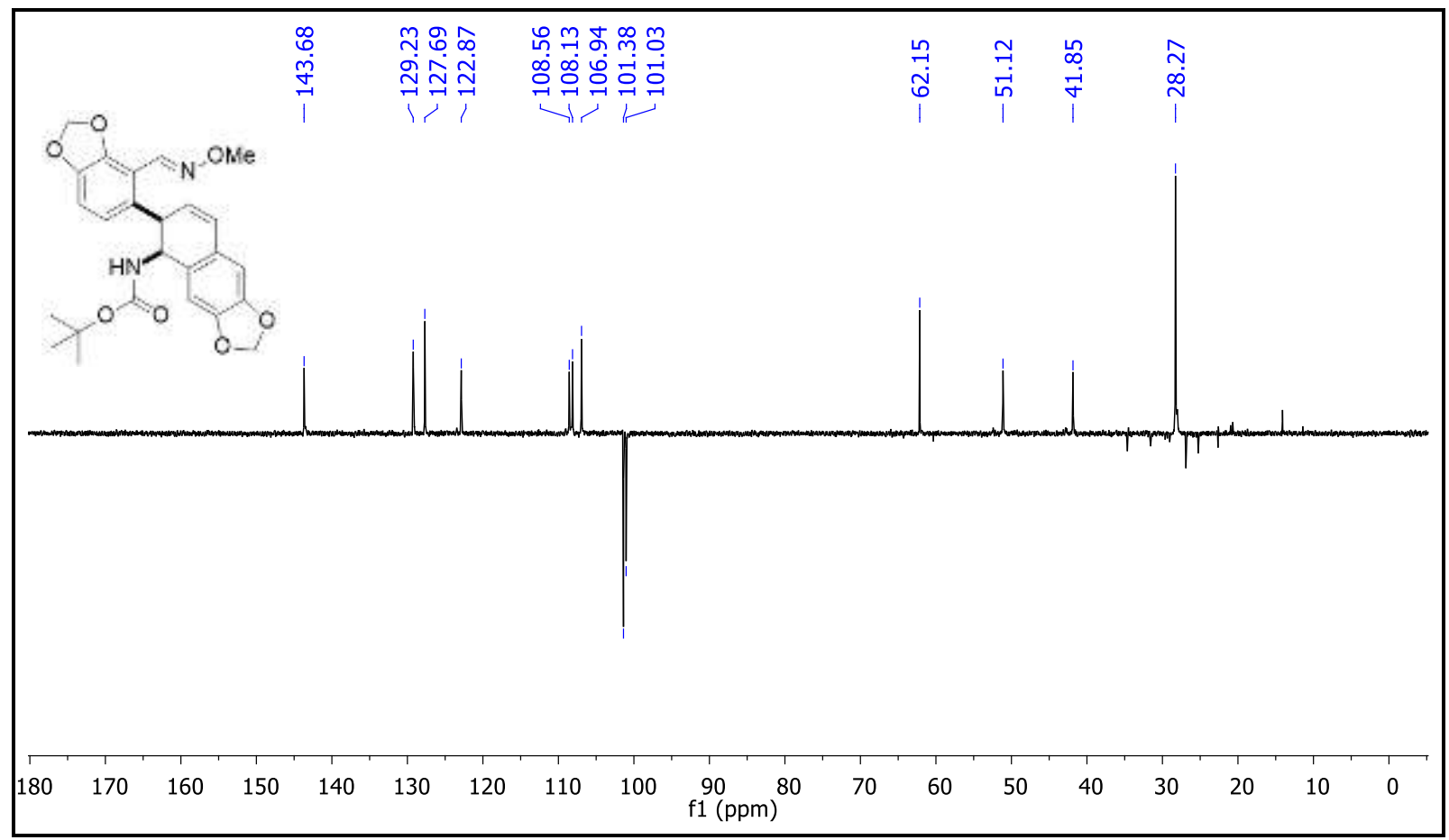


${ }^{1} \mathrm{H}$ and ${ }^{13} \mathrm{C}\left\{{ }^{1} \mathrm{H}\right\}$ NMR Spectra of Compound 3we $\left(\mathrm{CDCl}_{3}, 400 \mathrm{MHz}\right)$.
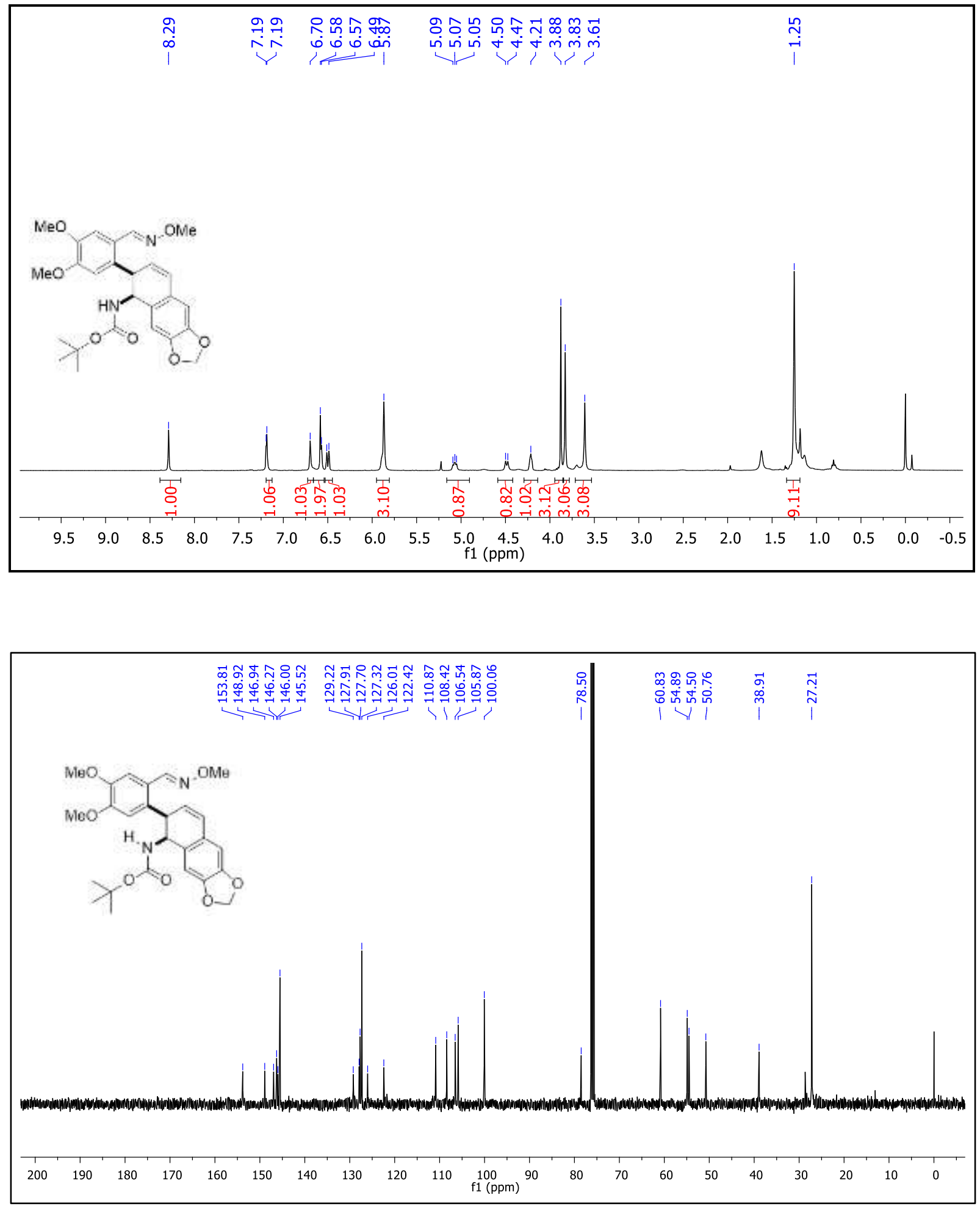
DEPT (135) NMR Spectrum of Compound 3we ( $\left.\mathrm{CDCl}_{3}, 101 \mathrm{MHz}\right)$.

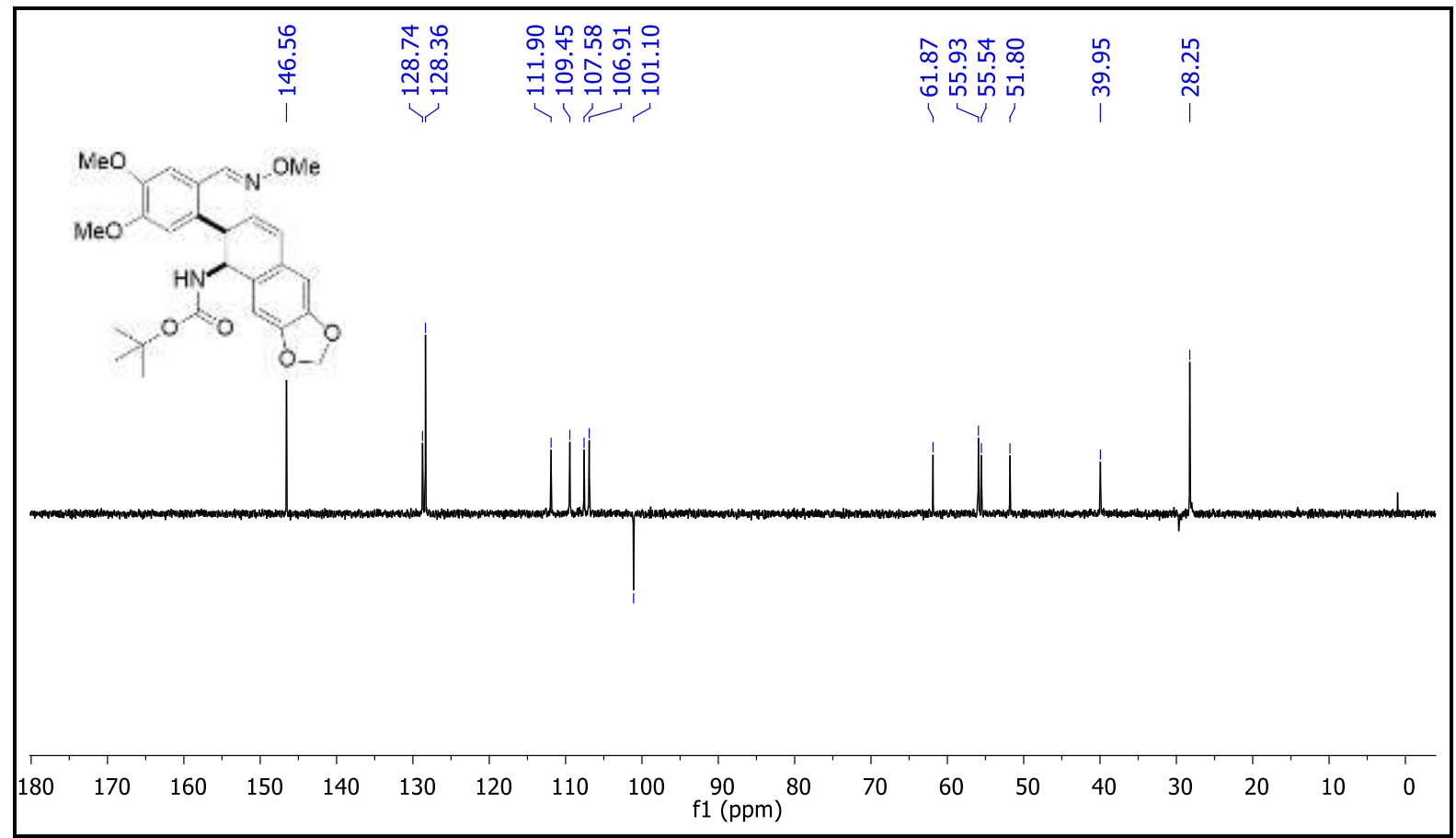


${ }^{1} \mathrm{H}$ and ${ }^{13} \mathrm{C}\left\{{ }^{1} \mathrm{H}\right\}$ NMR Spectra of Compound 3wf $\left(\mathrm{CDCl}_{3}, 400 \mathrm{MHz}\right)$.
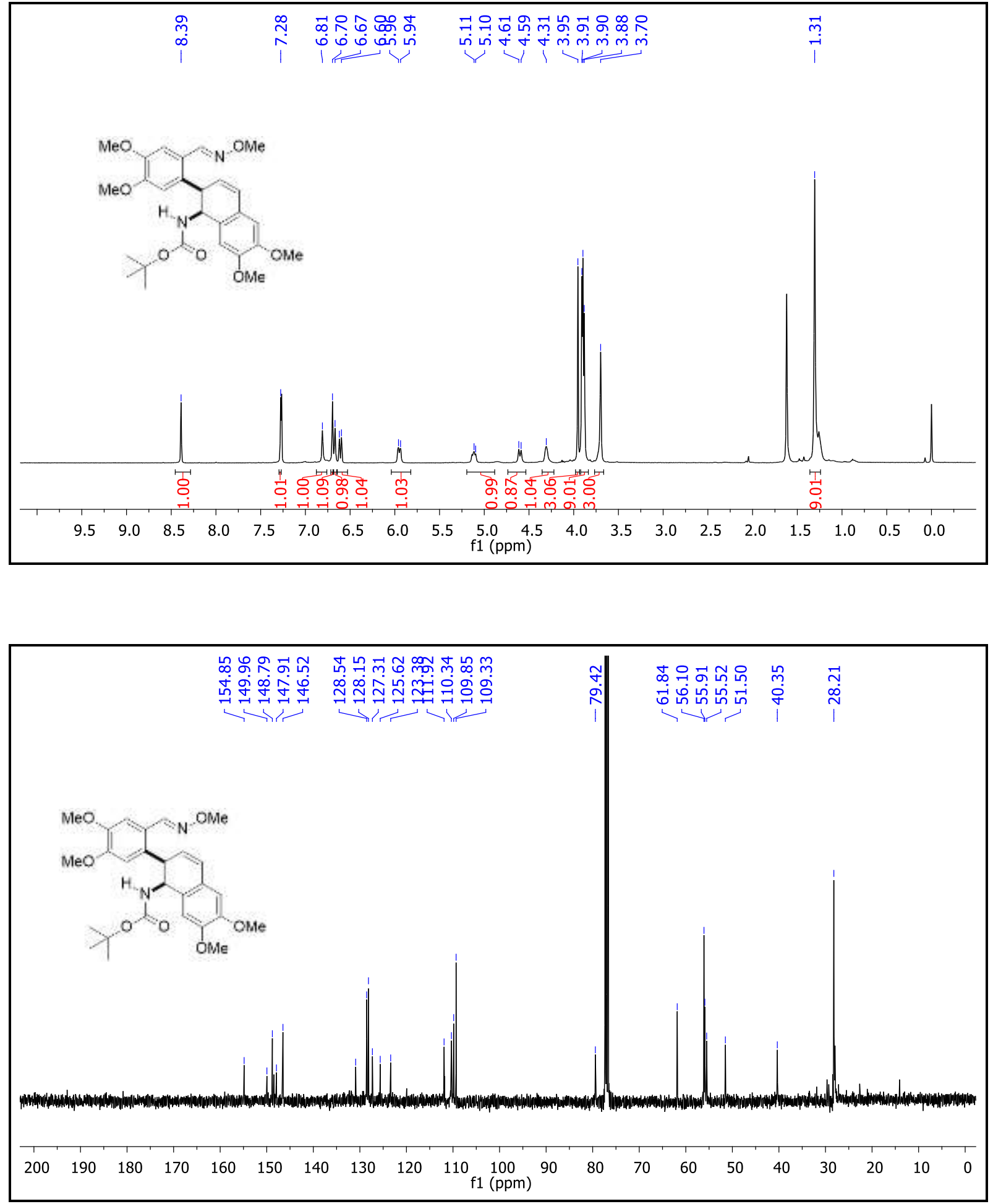
DEPT (135) NMR Spectrum of Compound $\mathbf{3 w f}\left(\mathrm{CDCl}_{3}, 101 \mathrm{MHz}\right)$.

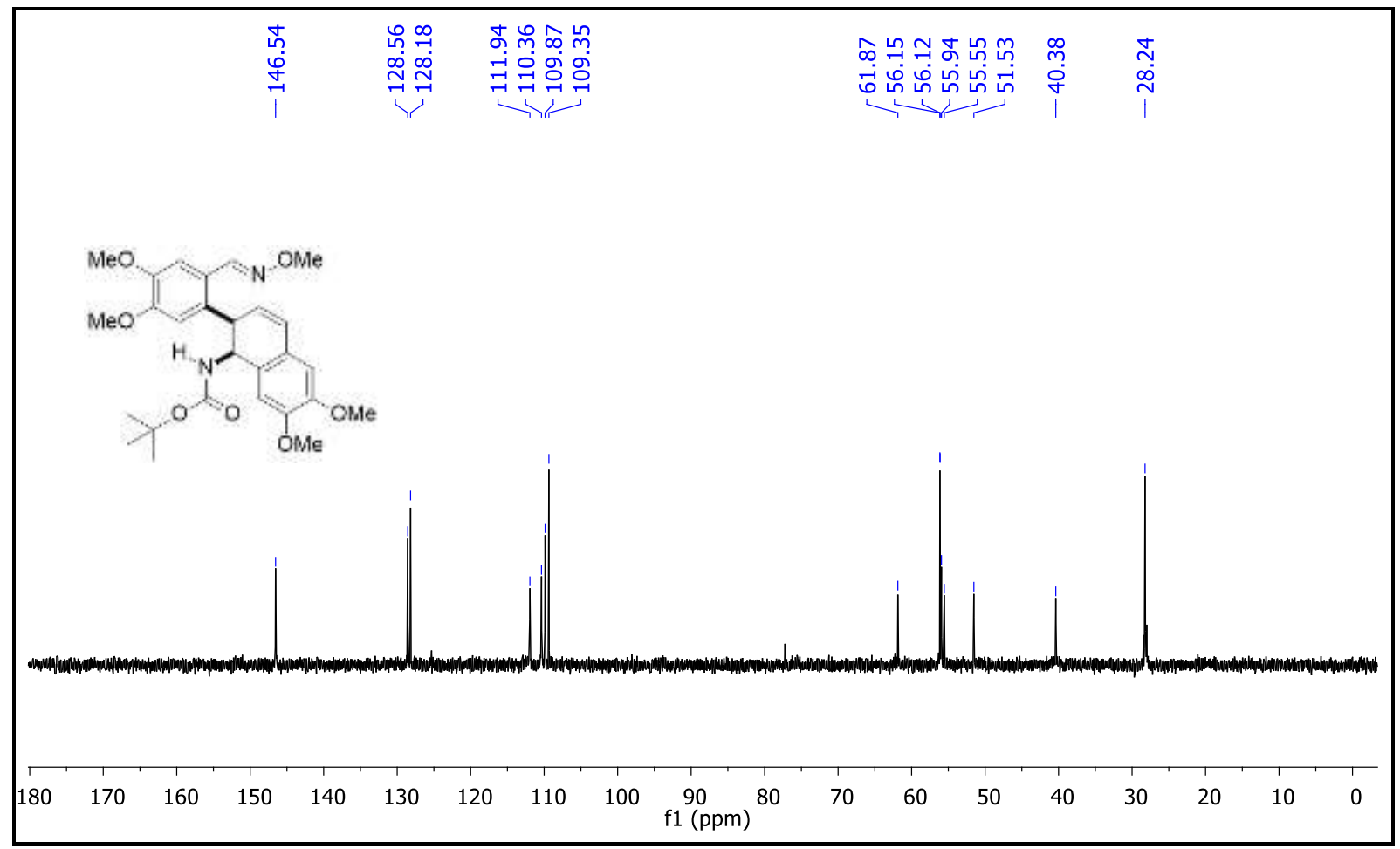


${ }^{1} \mathrm{H}$ and ${ }^{13} \mathrm{C}\left\{{ }^{1} \mathrm{H}\right\}$ NMR Spectra of Compound 3ye $\left(\mathrm{CDCl}_{3}, 400 \mathrm{MHz}\right)$.
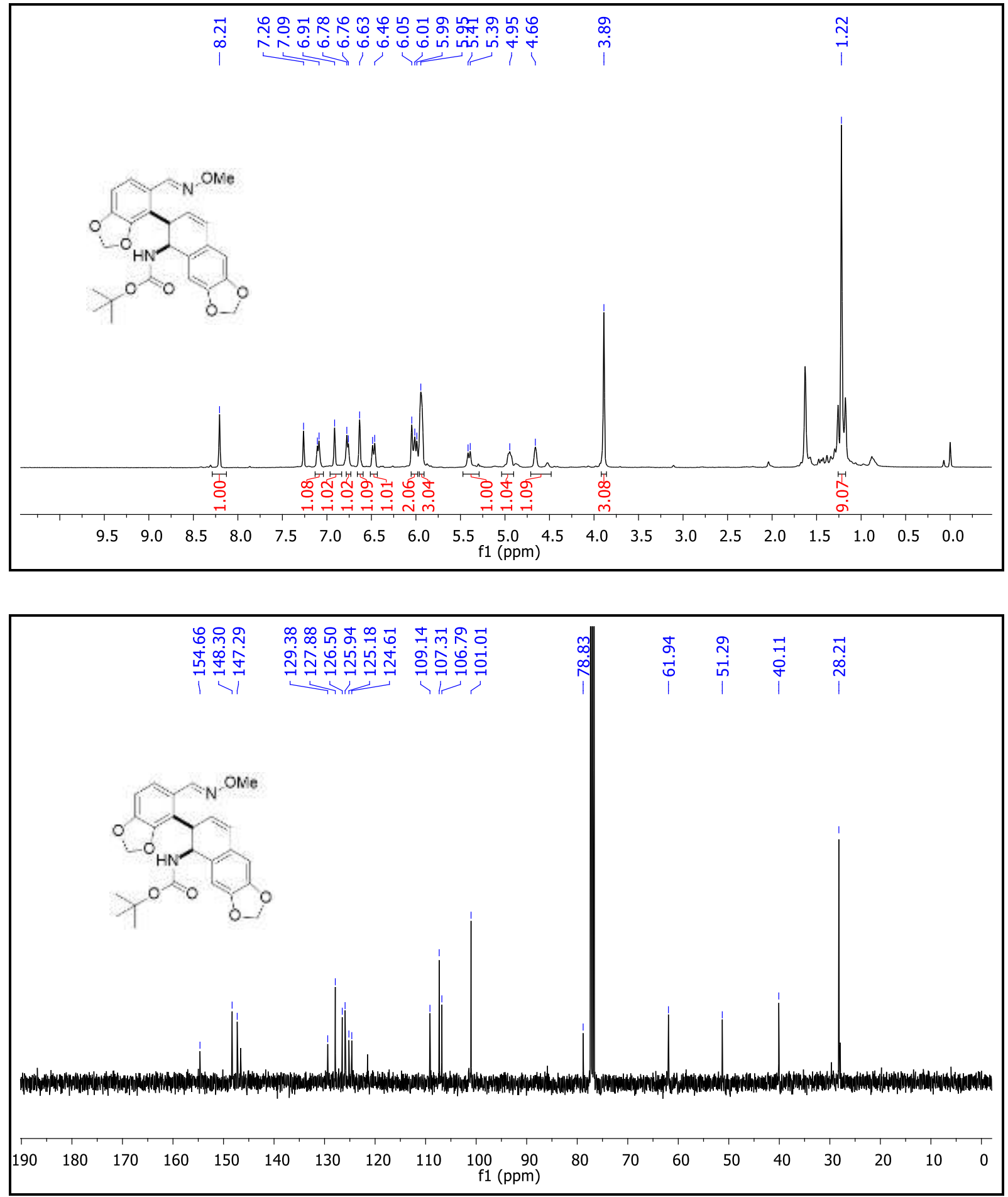
DEPT (135) NMR Spectrum of Compound 3ye $\left(\mathrm{CDCl}_{3}, 101 \mathrm{MHz}\right)$.

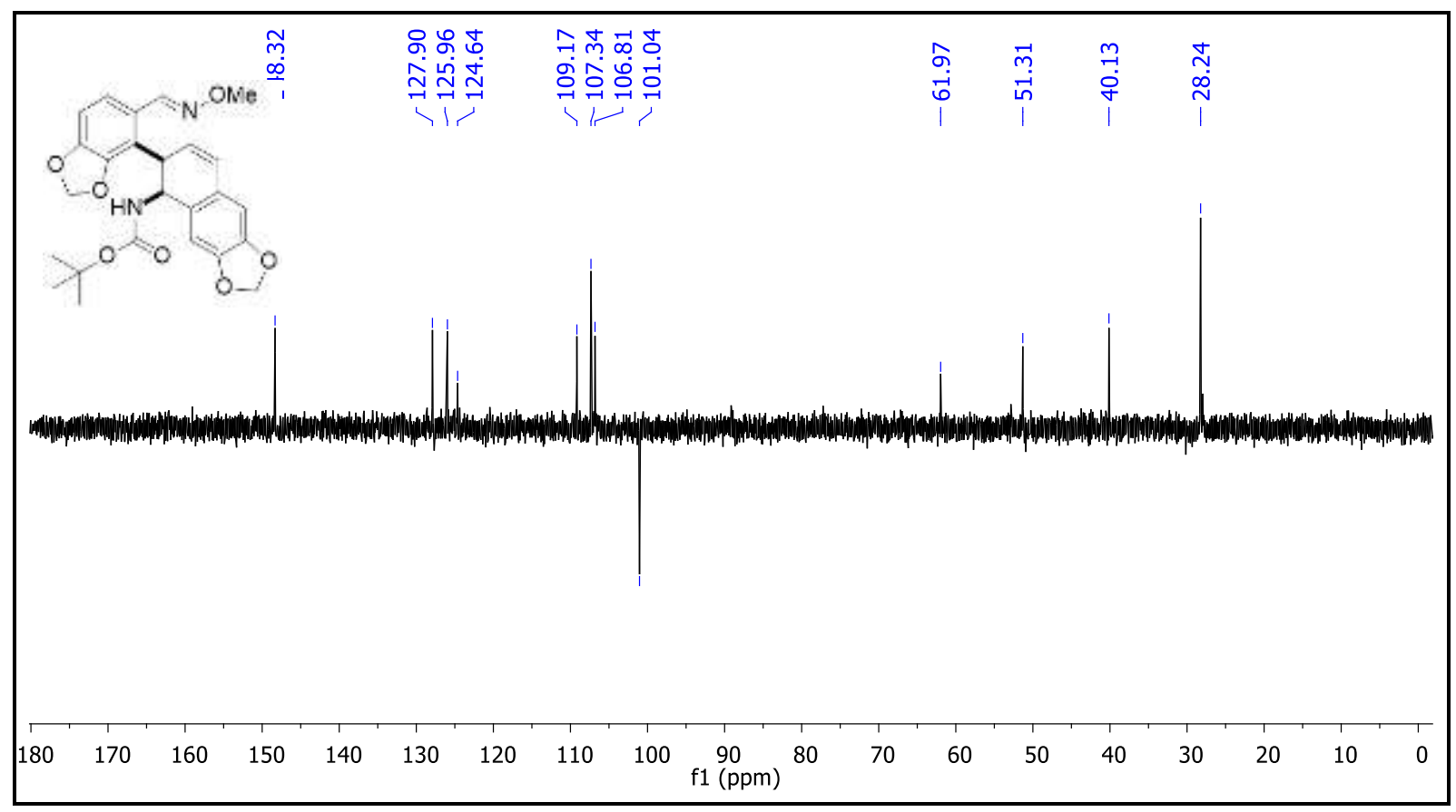


${ }^{1} \mathrm{H}$ and ${ }^{13} \mathrm{C}\left\{{ }^{1} \mathrm{H}\right\}$ NMR Spectra of Compound $\mathbf{3 y f}\left(\mathrm{CDCl}_{3}, 400 \mathrm{MHz}\right)$.
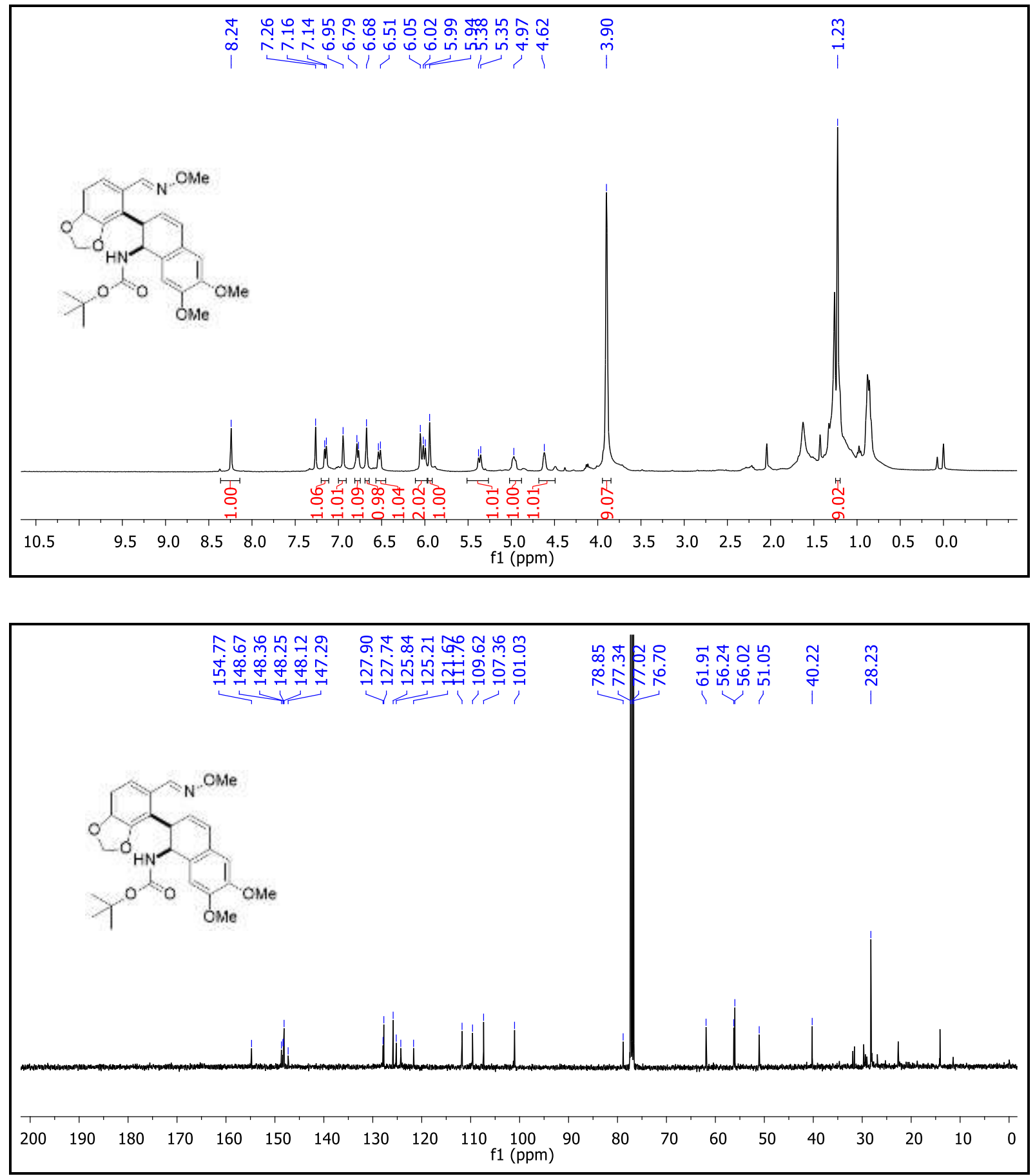
DEPT (135) NMR Spectrum of Compound $\mathbf{3 y f}\left(\mathrm{CDCl}_{3}, 101 \mathrm{MHz}\right)$.

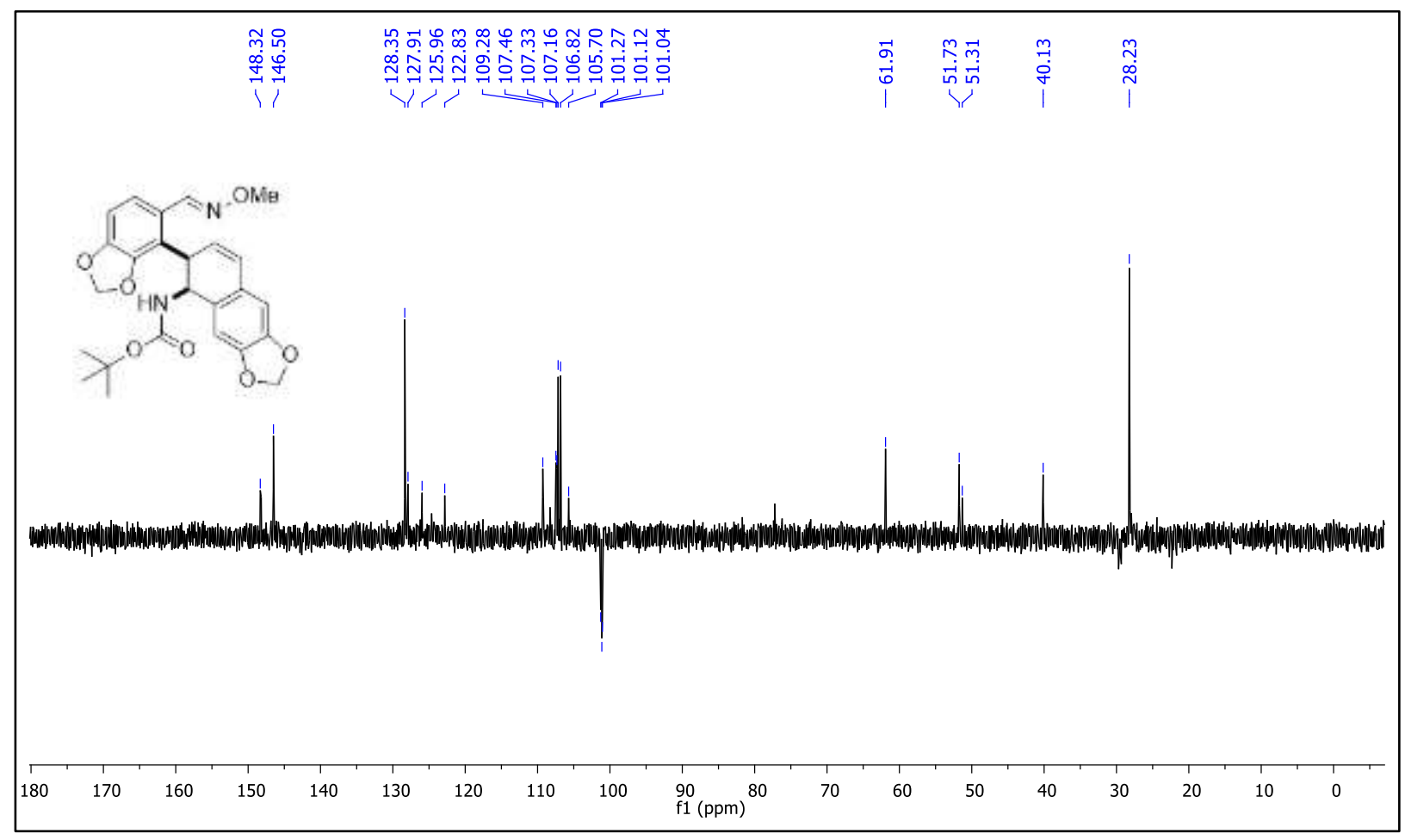


${ }^{1} \mathrm{H}$ and ${ }^{13} \mathrm{C}\left\{{ }^{1} \mathrm{H}\right\}$ NMR Spectra of Compound $4 \mathbf{a}\left(\mathrm{CDCl}_{3}, 400 \mathrm{MHz}\right)$.
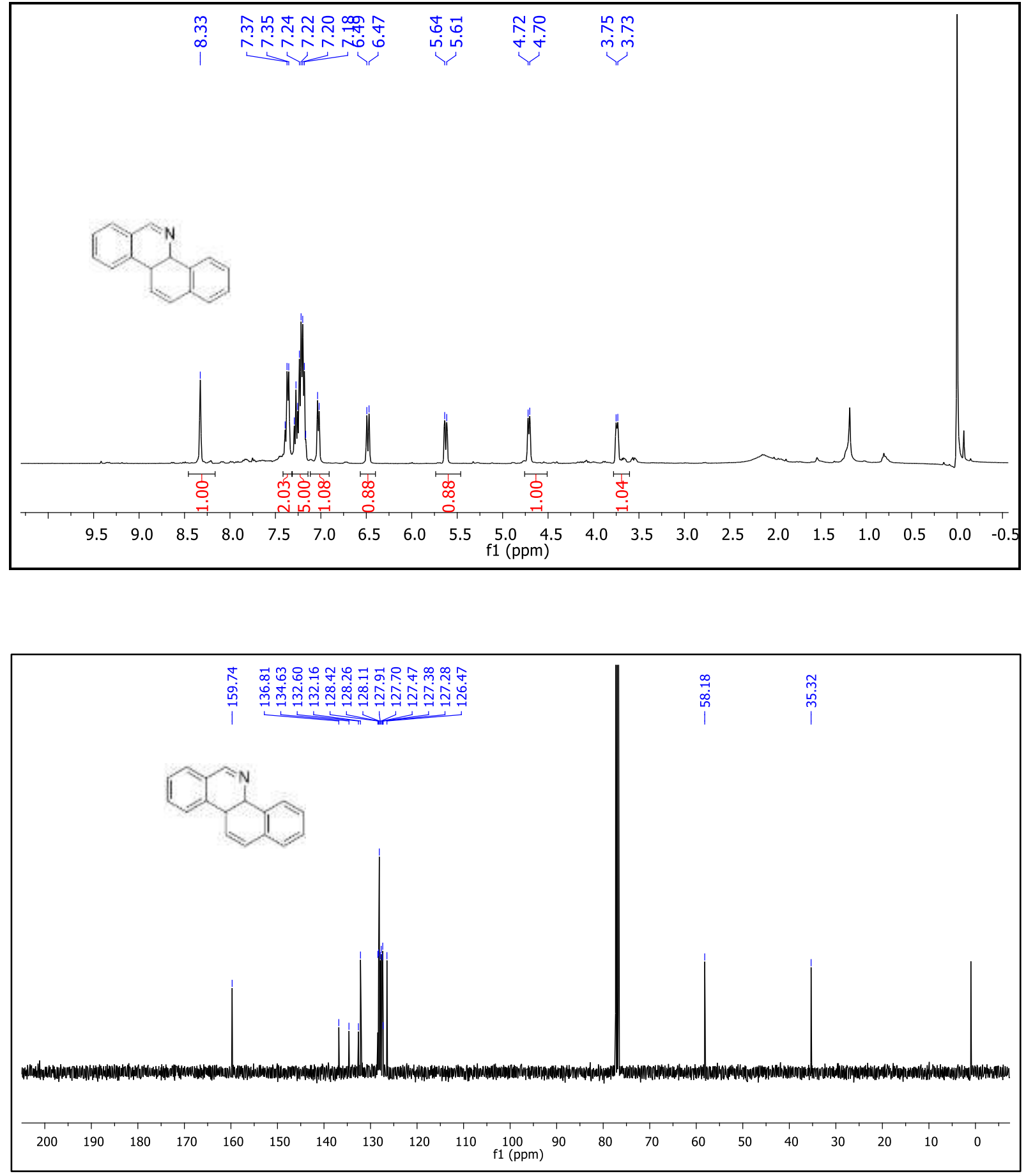
DEPT (135) NMR Spectrum of Compound $\mathbf{4 a}\left(\mathrm{CDCl}_{3}, 101 \mathrm{MHz}\right)$.

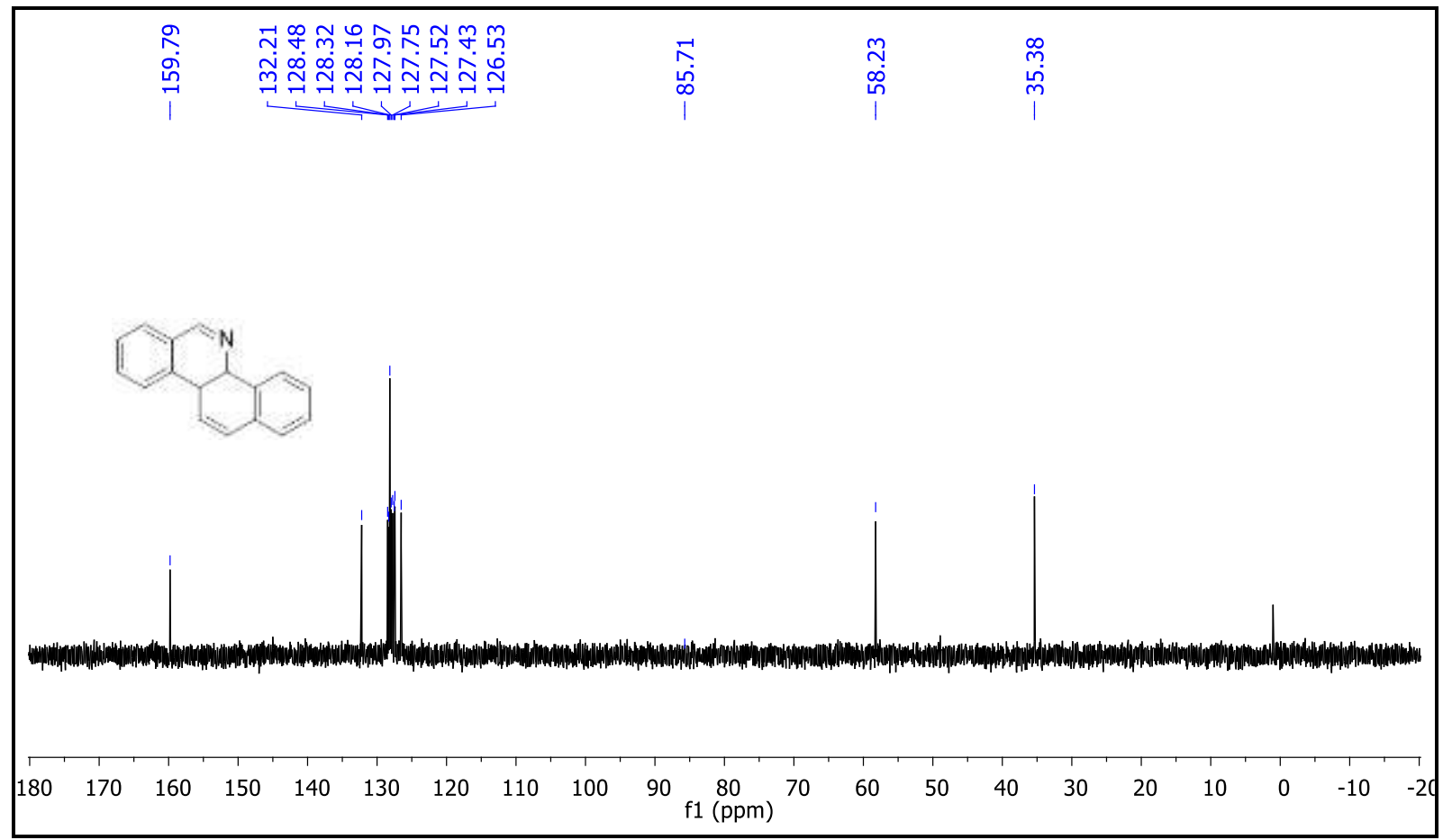


${ }^{1} \mathrm{H}$ and ${ }^{13} \mathrm{C}\left\{{ }^{1} \mathrm{H}\right\}$ NMR Spectra of Compound $\mathbf{4 b}\left(\mathrm{CDCl}_{3}, 400 \mathrm{MHz}\right)$.
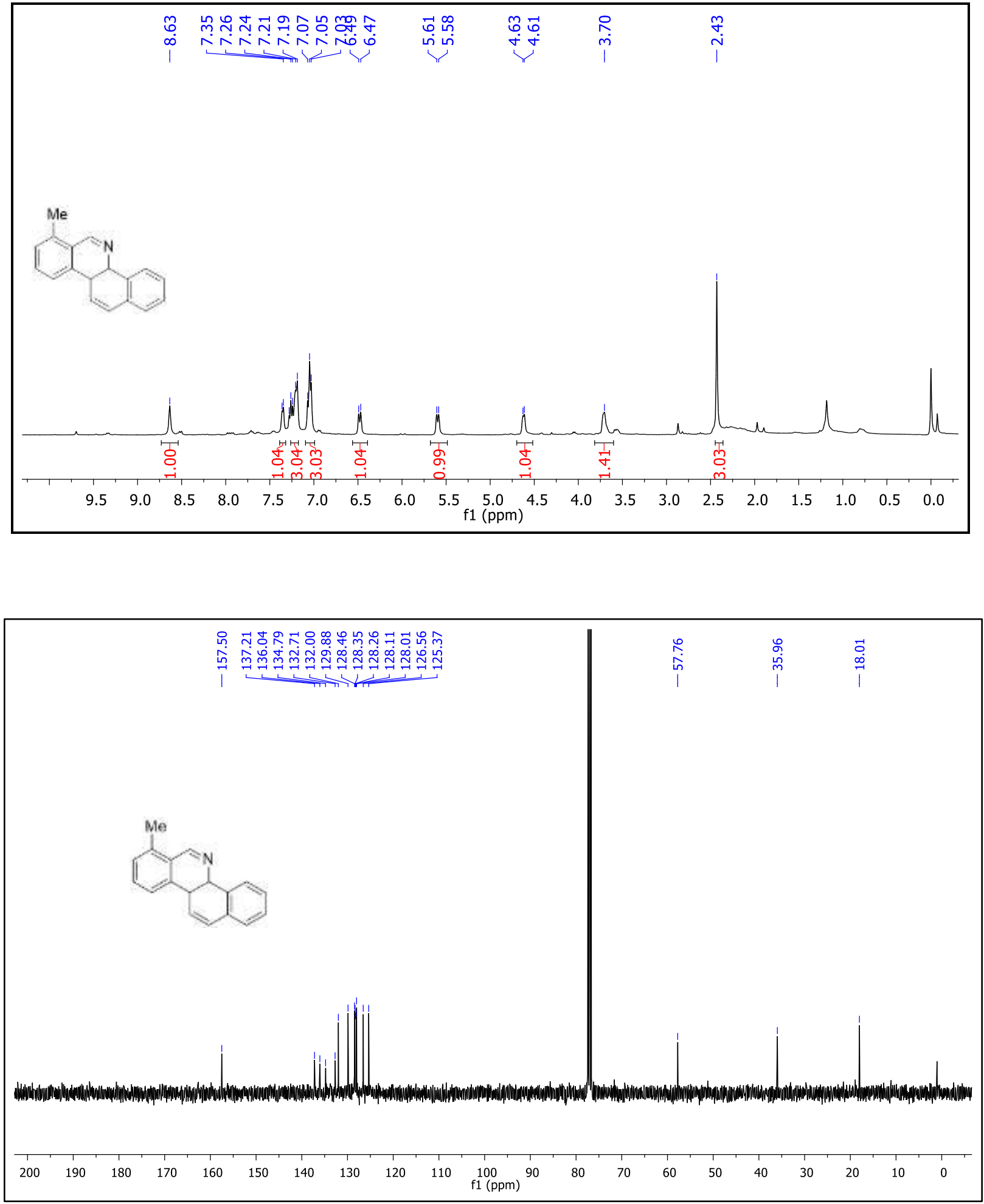
DEPT (135) NMR Spectrum of Compound $\mathbf{4 b}\left(\mathrm{CDCl}_{3}, 101 \mathrm{MHz}\right)$.

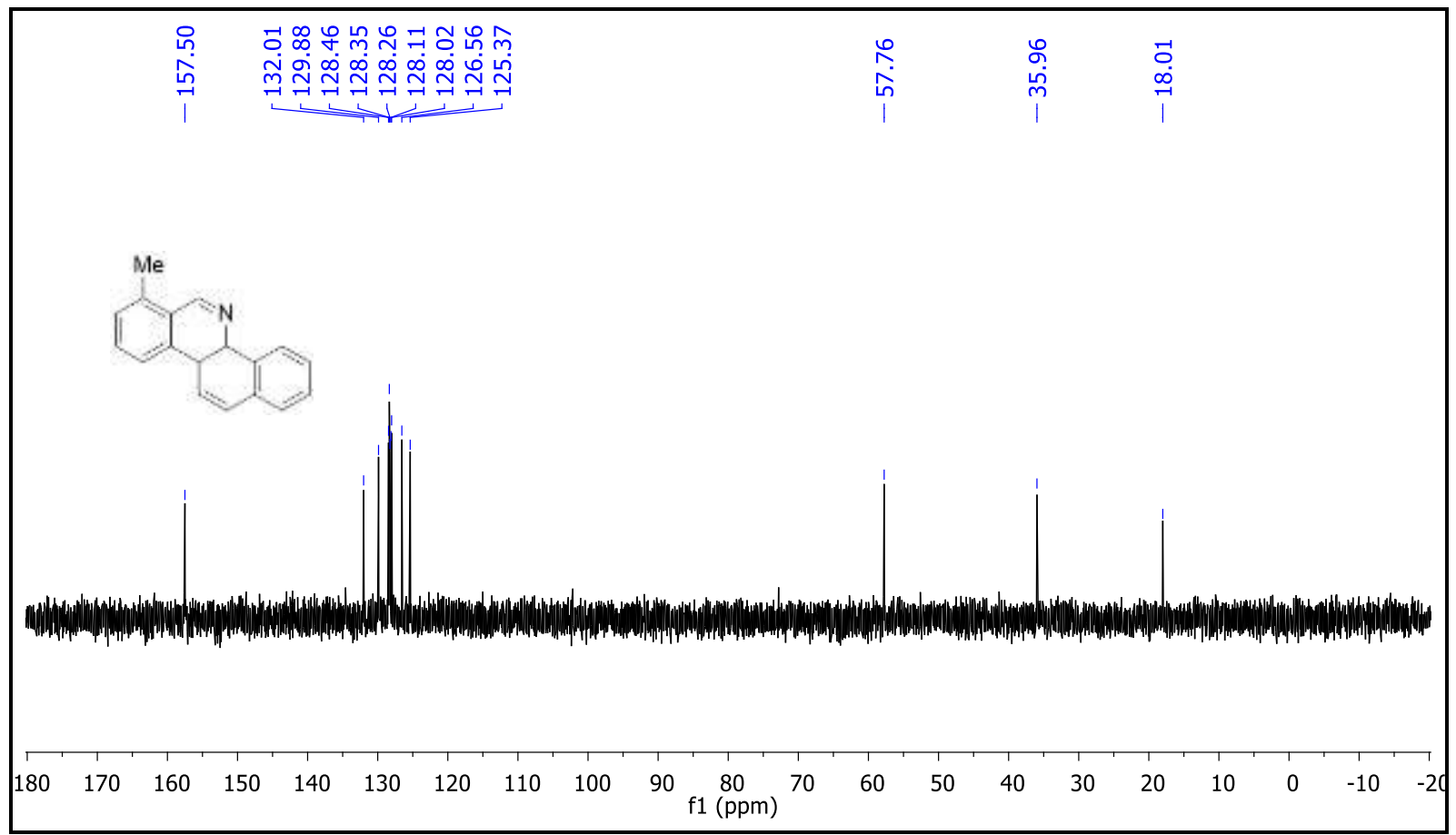


${ }^{1} \mathrm{H}$ and ${ }^{13} \mathrm{C}\left\{{ }^{1} \mathrm{H}\right\}$ NMR Spectra of Compound $\mathbf{4 c}\left(\mathrm{CDCl}_{3}, 400 \mathrm{MHz}\right)$.
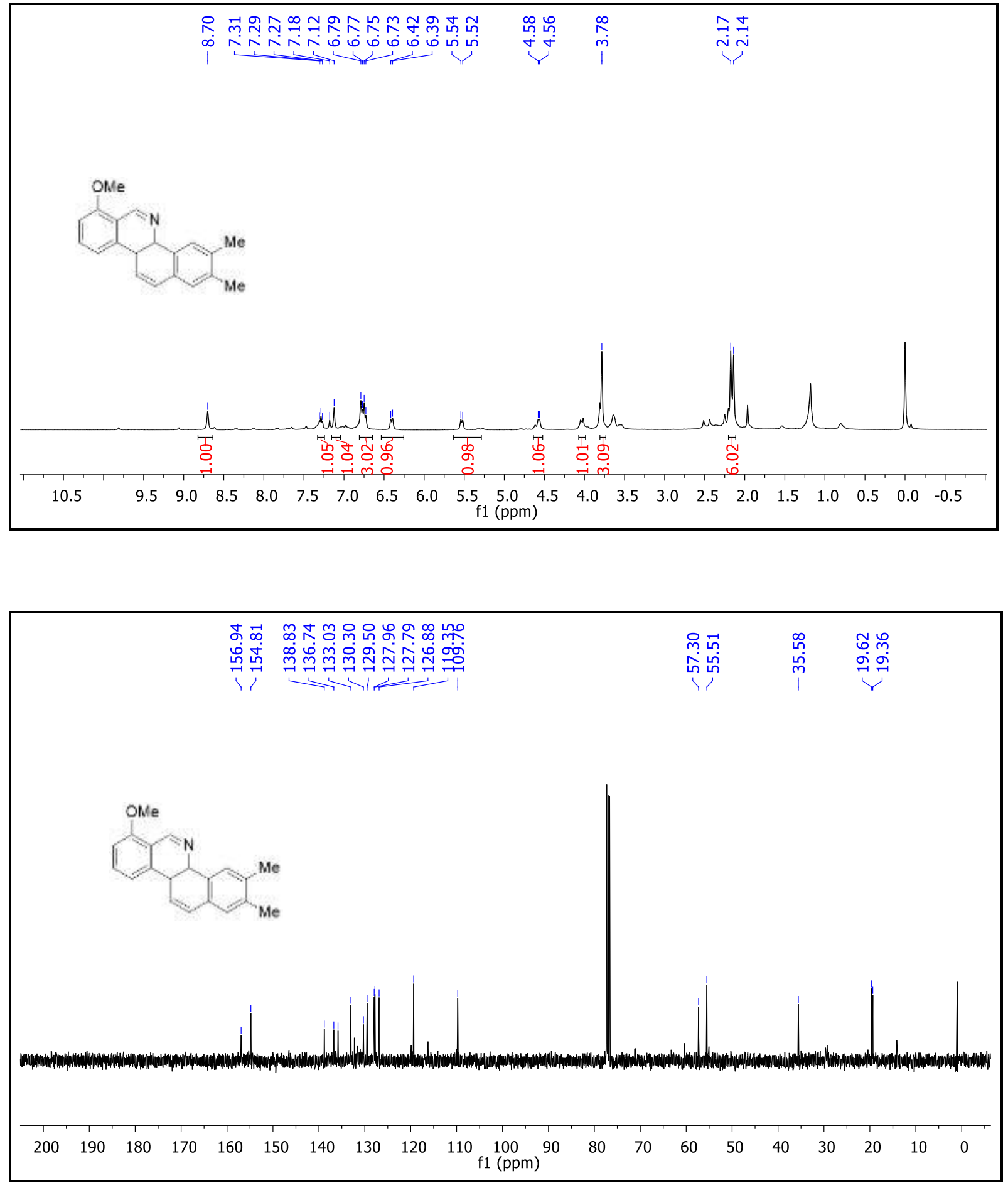
DEPT (135) NMR Spectrum of Compound $4 \mathbf{c}\left(\mathrm{CDCl}_{3}, 101 \mathrm{MHz}\right)$.

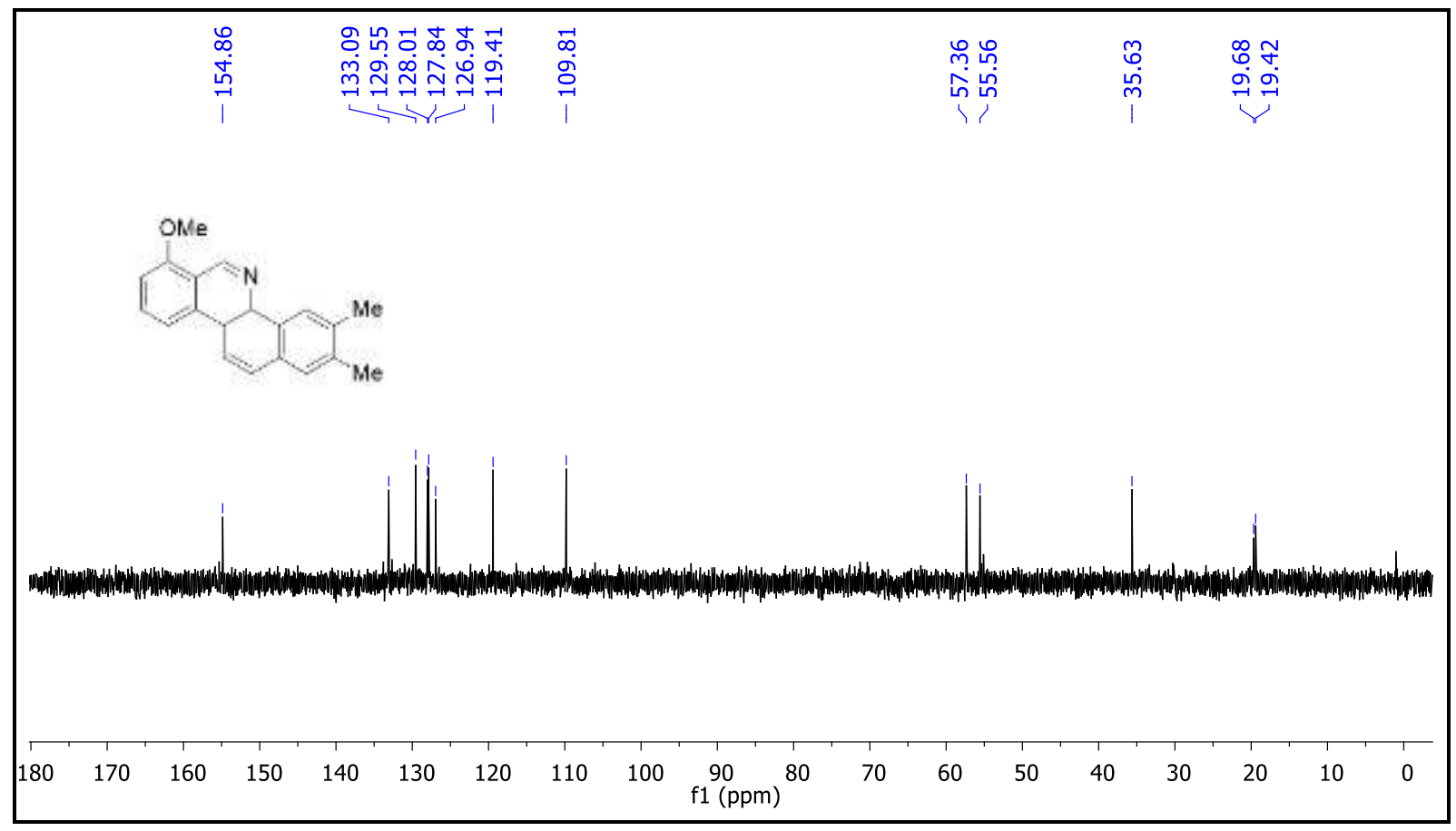


${ }^{1} \mathrm{H}$ and ${ }^{13} \mathrm{C}\left\{{ }^{1} \mathrm{H}\right\}$ NMR Spectra of Compound $\mathbf{4 d}\left(\mathrm{CDCl}_{3}, 400 \mathrm{MHz}\right)$.
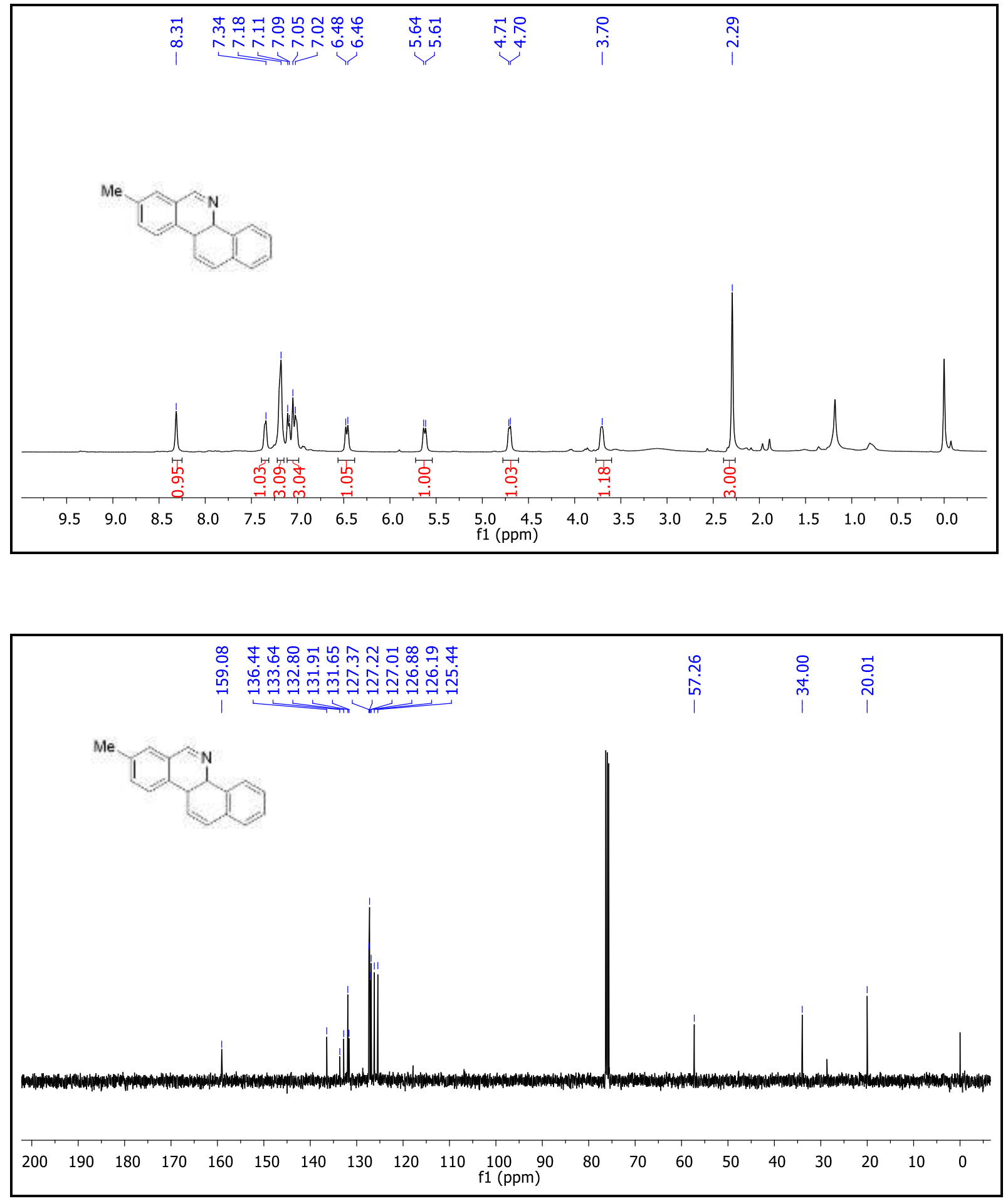
DEPT (135) NMR Spectrum of Compound $\mathbf{4 d}\left(\mathrm{CDCl}_{3}, 101 \mathrm{MHz}\right)$.

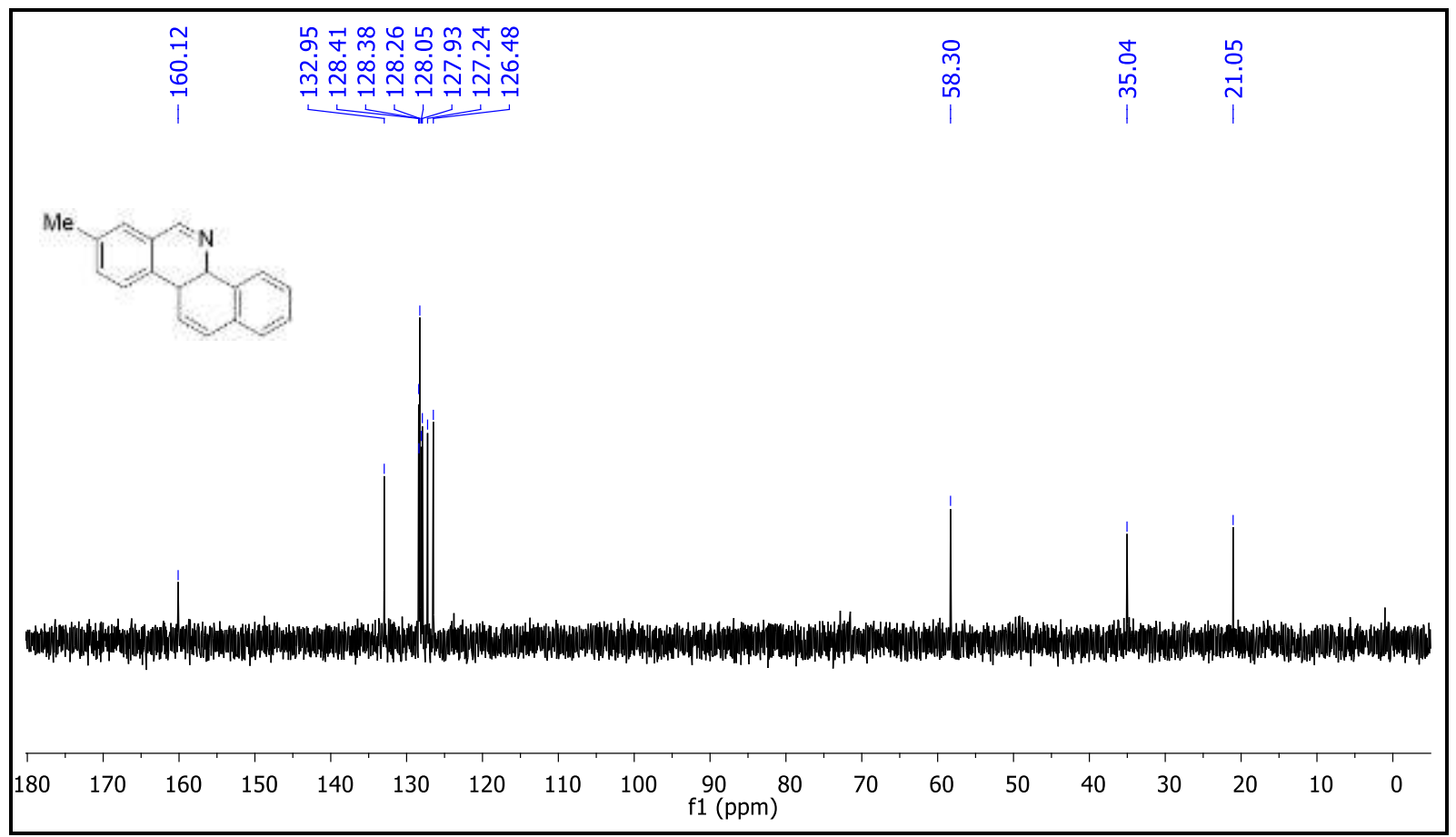


${ }^{1} \mathrm{H}$ and ${ }^{13} \mathrm{C}\left\{{ }^{1} \mathrm{H}\right\}$ NMR Spectra of Compound $4 \mathbf{e}\left(\mathrm{CDCl}_{3}, 400 \mathrm{MHz}\right)$.
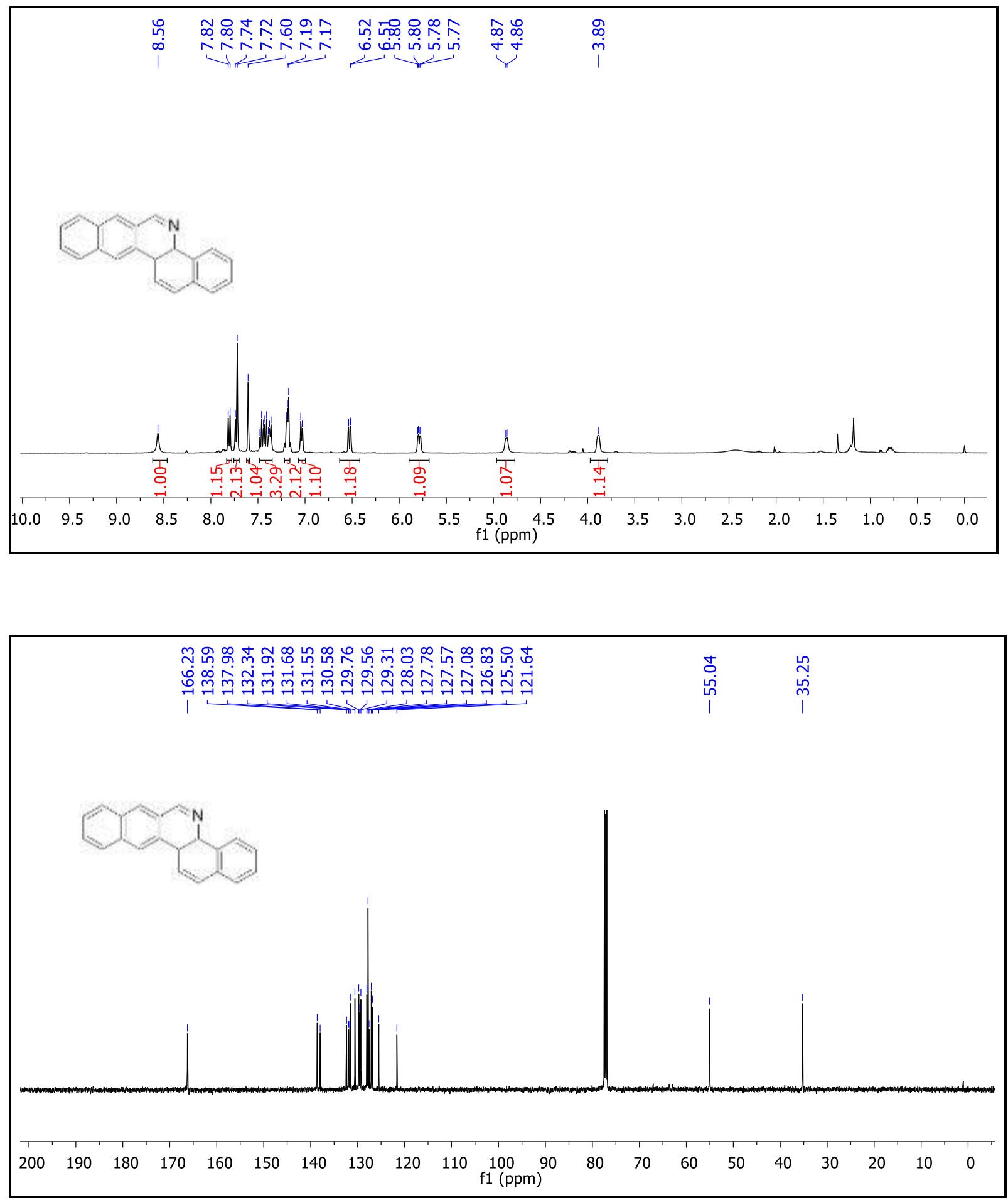
DEPT (135) NMR Spectrum of Compound $4 \mathbf{e}\left(\mathrm{CDCl}_{3}, 101 \mathrm{MHz}\right)$.

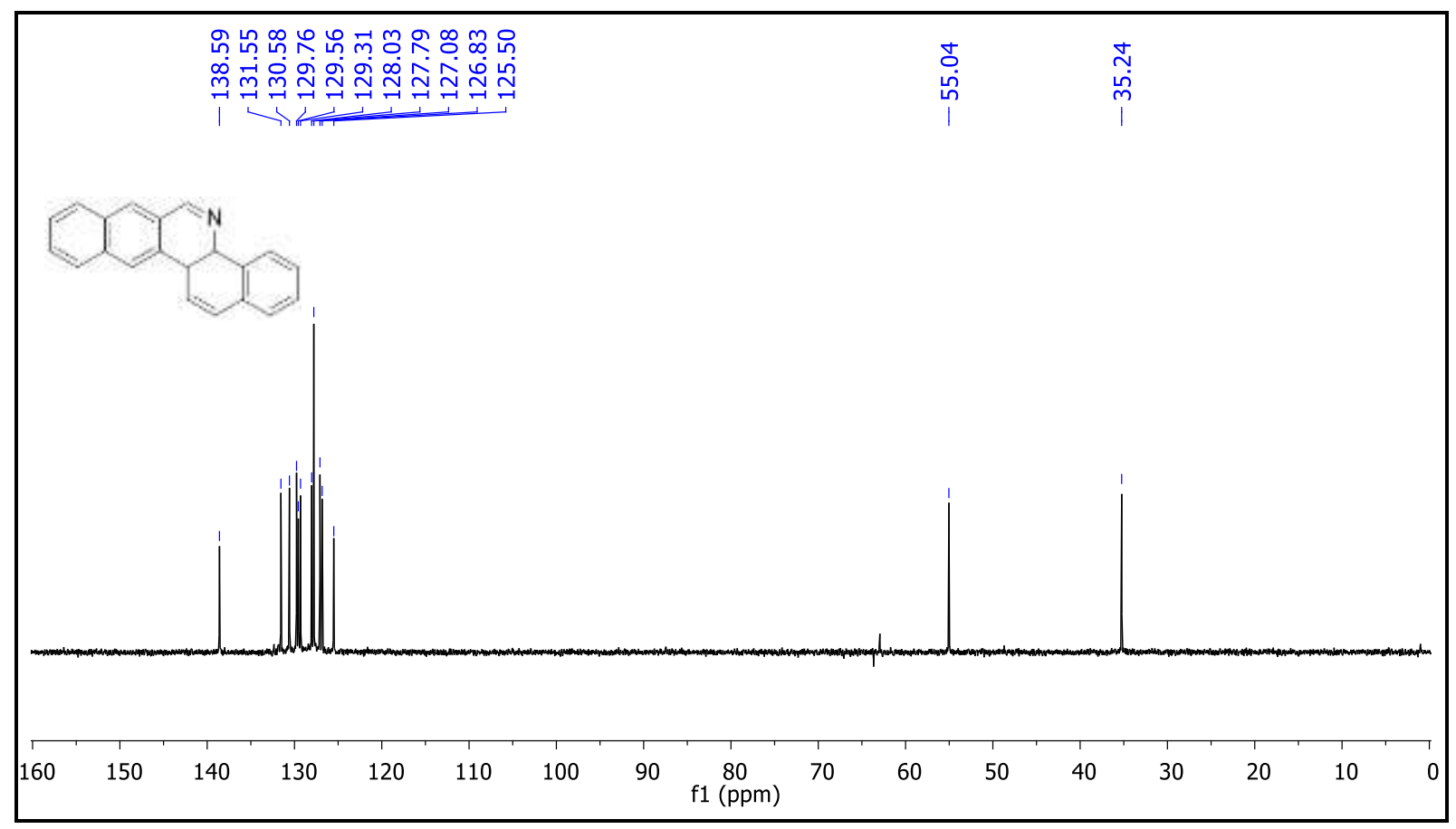


${ }^{1} \mathrm{H}$ and ${ }^{13} \mathrm{C}\left\{{ }^{1} \mathrm{H}\right\}$ NMR Spectra of Compound $\mathbf{4 f}\left(\mathrm{CDCl}_{3}, 400 \mathrm{MHz}\right)$.
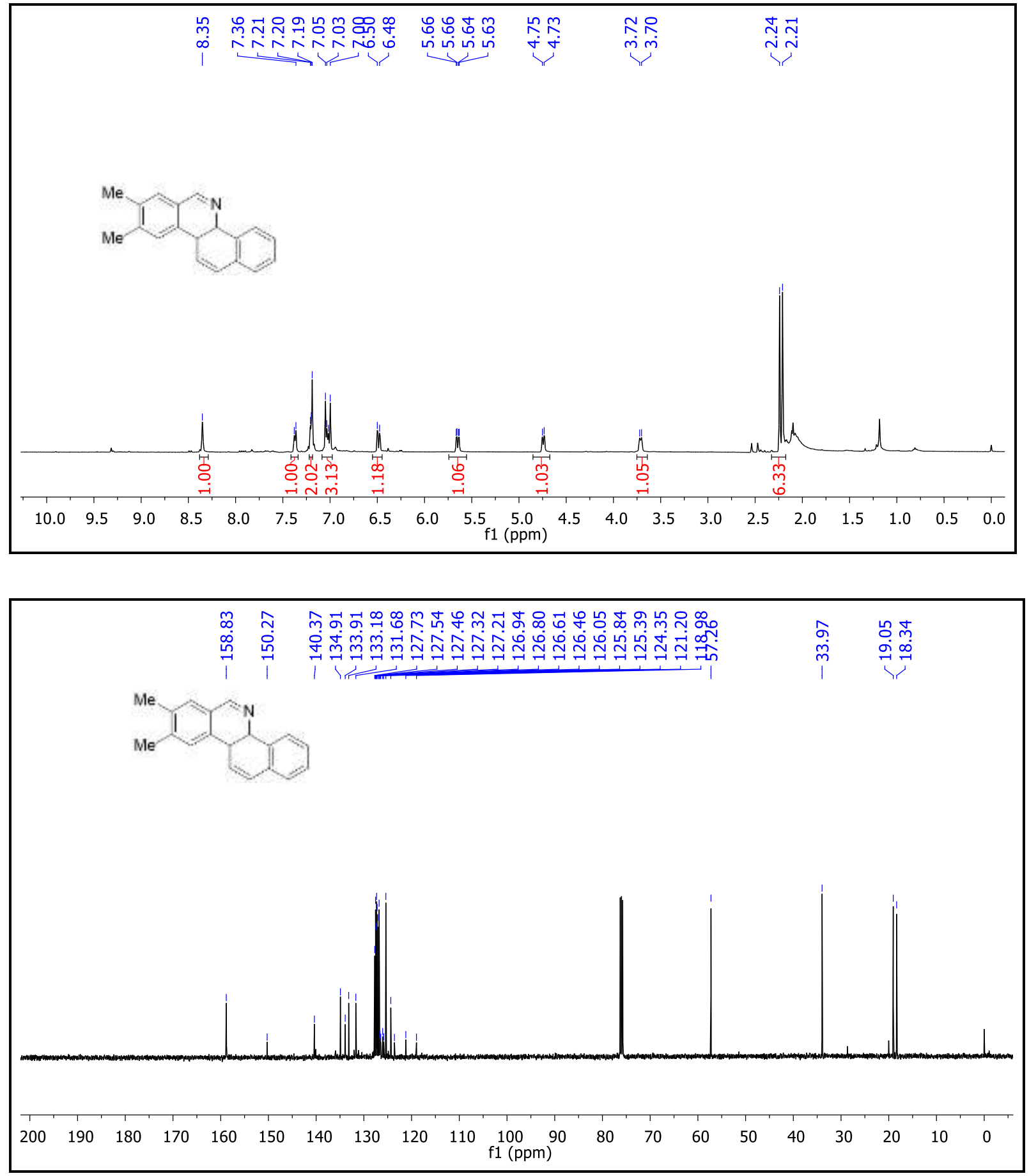
DEPT (135) NMR Spectrum of Compound $\mathbf{4 f}\left(\mathrm{CDCl}_{3}, 126 \mathrm{MHz}\right)$.

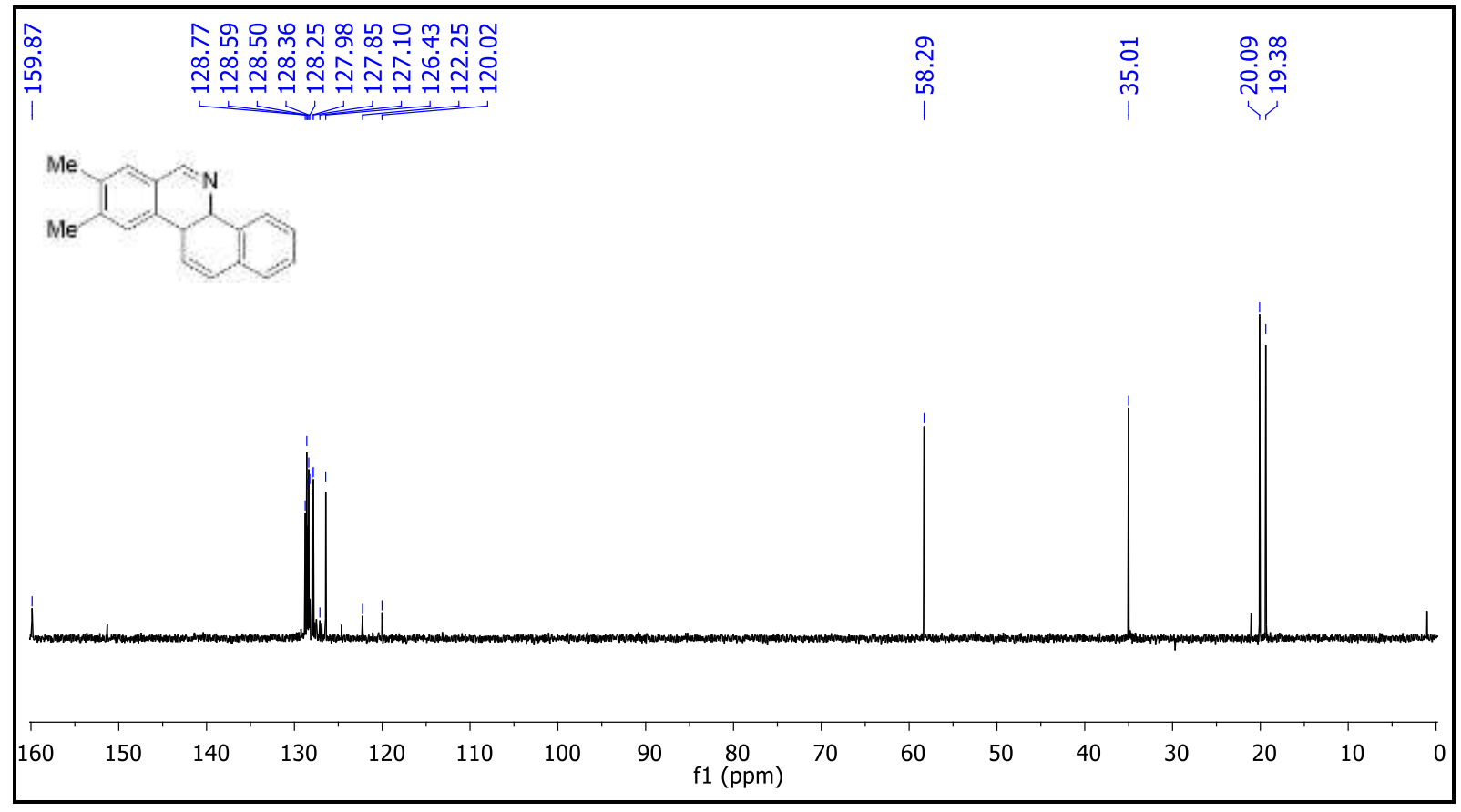


${ }^{1} \mathrm{H}$ and ${ }^{13} \mathrm{C}\left\{{ }^{1} \mathrm{H}\right\}$ NMR Spectra of Compound $4 \mathrm{~g}\left(\mathrm{CDCl}_{3}, 400 \mathrm{MHz}\right)$.
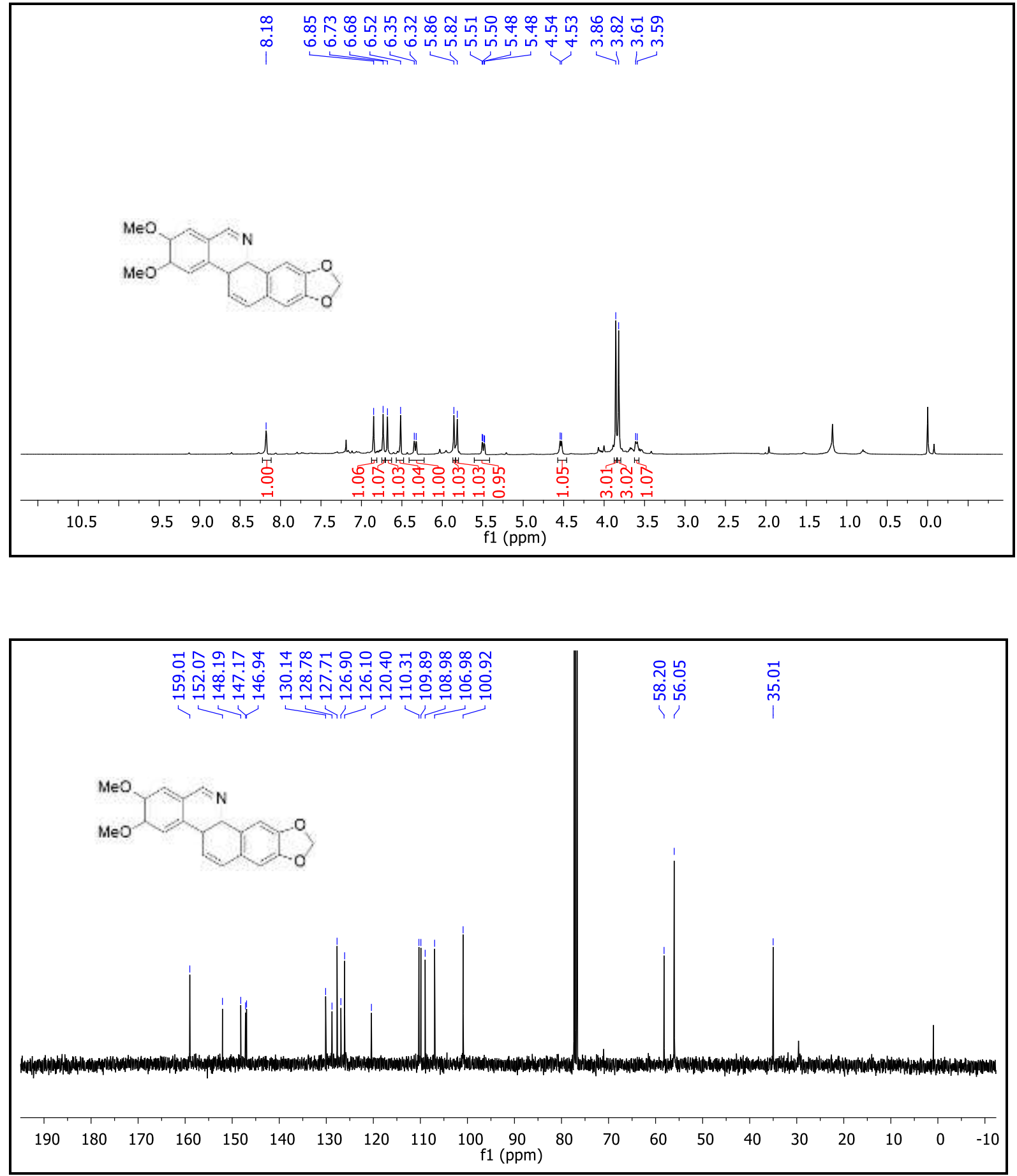
DEPT (135) NMR Spectrum of Compound $\mathbf{4 g}\left(\mathrm{CDCl}_{3}, 101 \mathrm{MHz}\right)$.

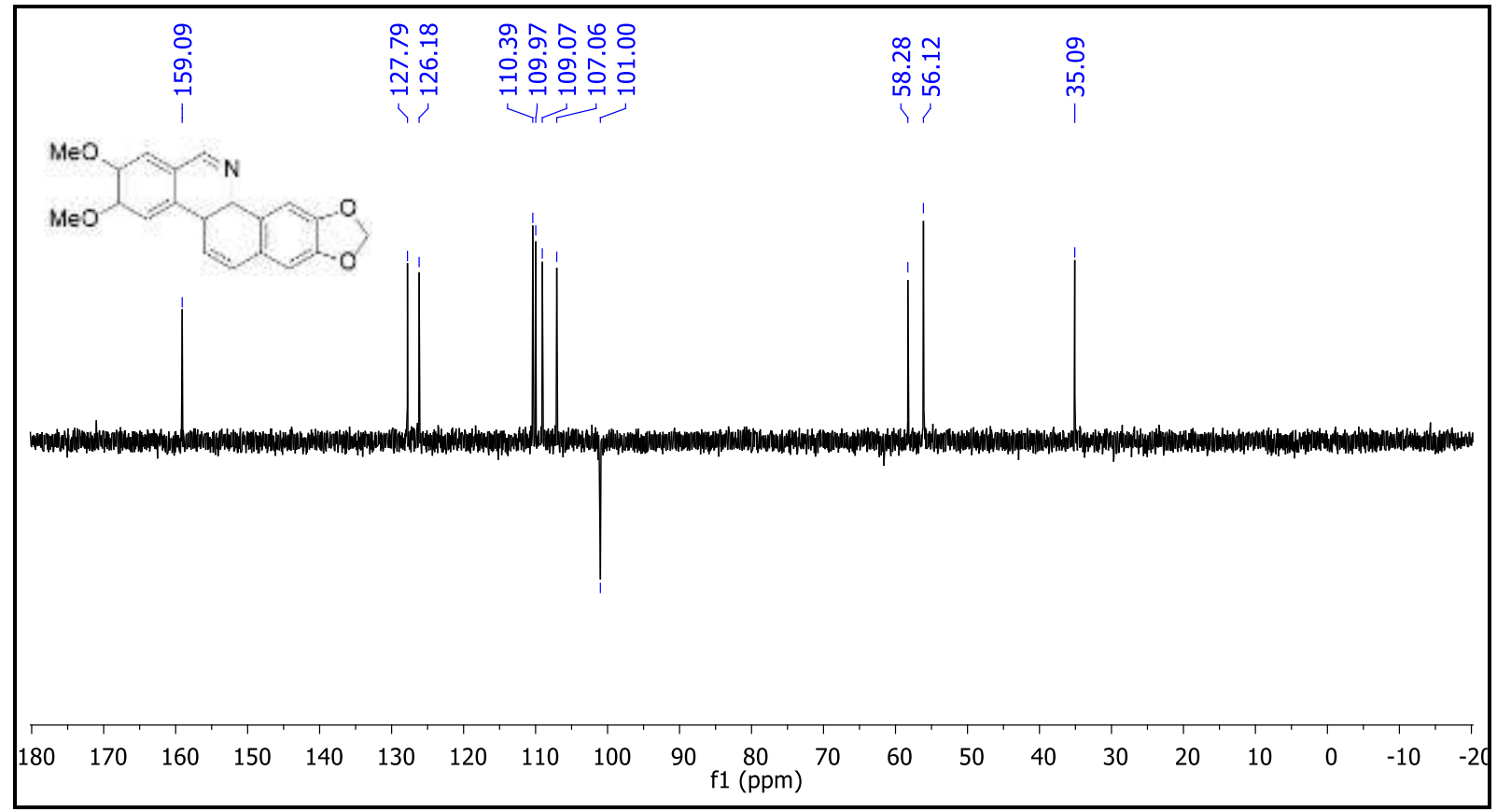


${ }^{1} \mathrm{H}$ and ${ }^{13} \mathrm{C}\left\{{ }^{1} \mathrm{H}\right\}$ NMR Spectra of Compound $\mathbf{4 h}\left(\mathrm{CDCl}_{3}, 400 \mathrm{MHz}\right)$.
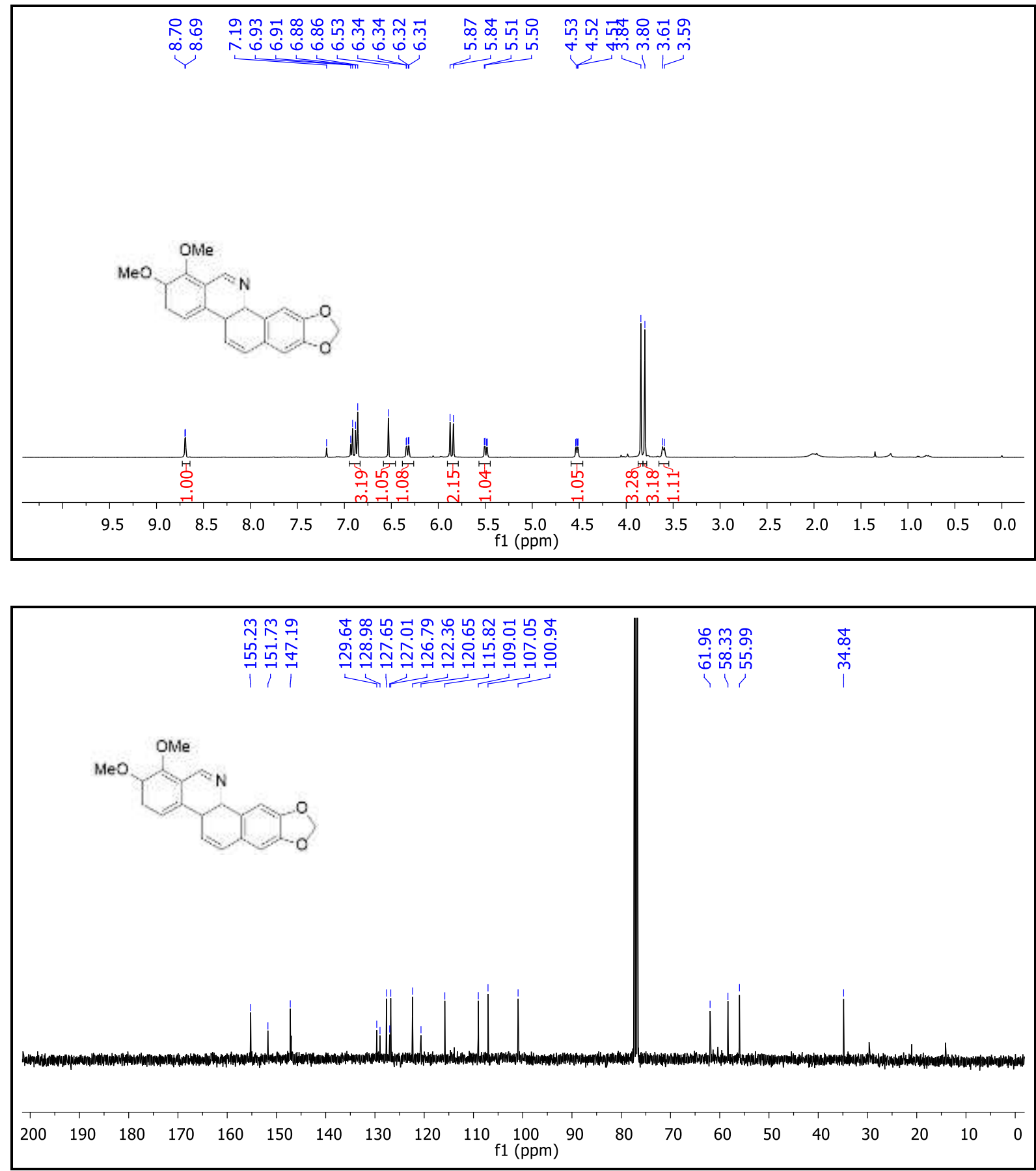
DEPT (135) NMR Spectrum of Compound $\mathbf{4 h}\left(\mathrm{CDCl}_{3}, 101 \mathrm{MHz}\right)$.

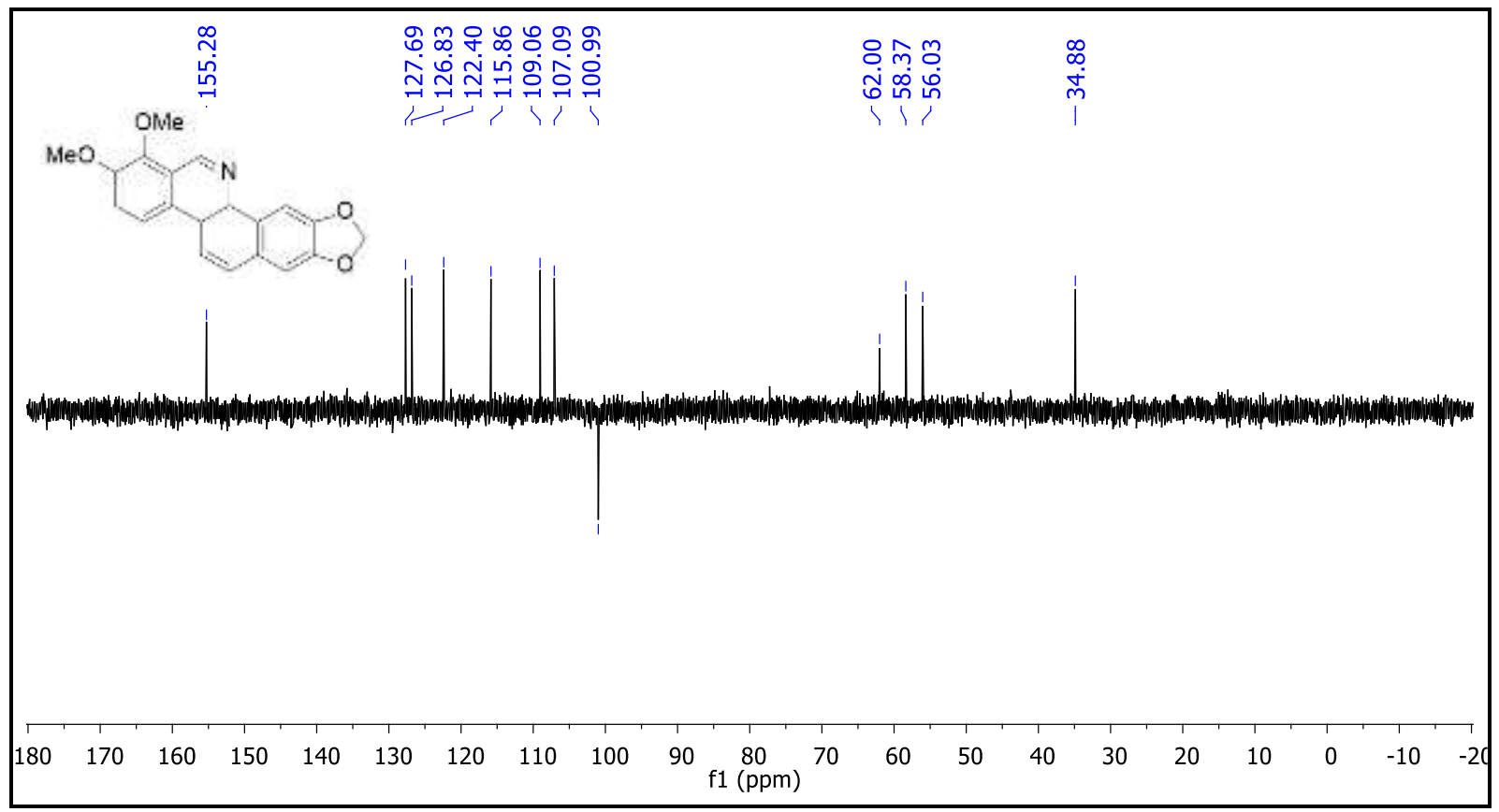


${ }^{1} \mathrm{H}$ and ${ }^{13} \mathrm{C}\left\{{ }^{1} \mathrm{H}\right\}$ NMR Spectra of Compound $\mathbf{5 a}\left(\mathrm{CDCl}_{3}, 400 \mathrm{MHz}\right)$.
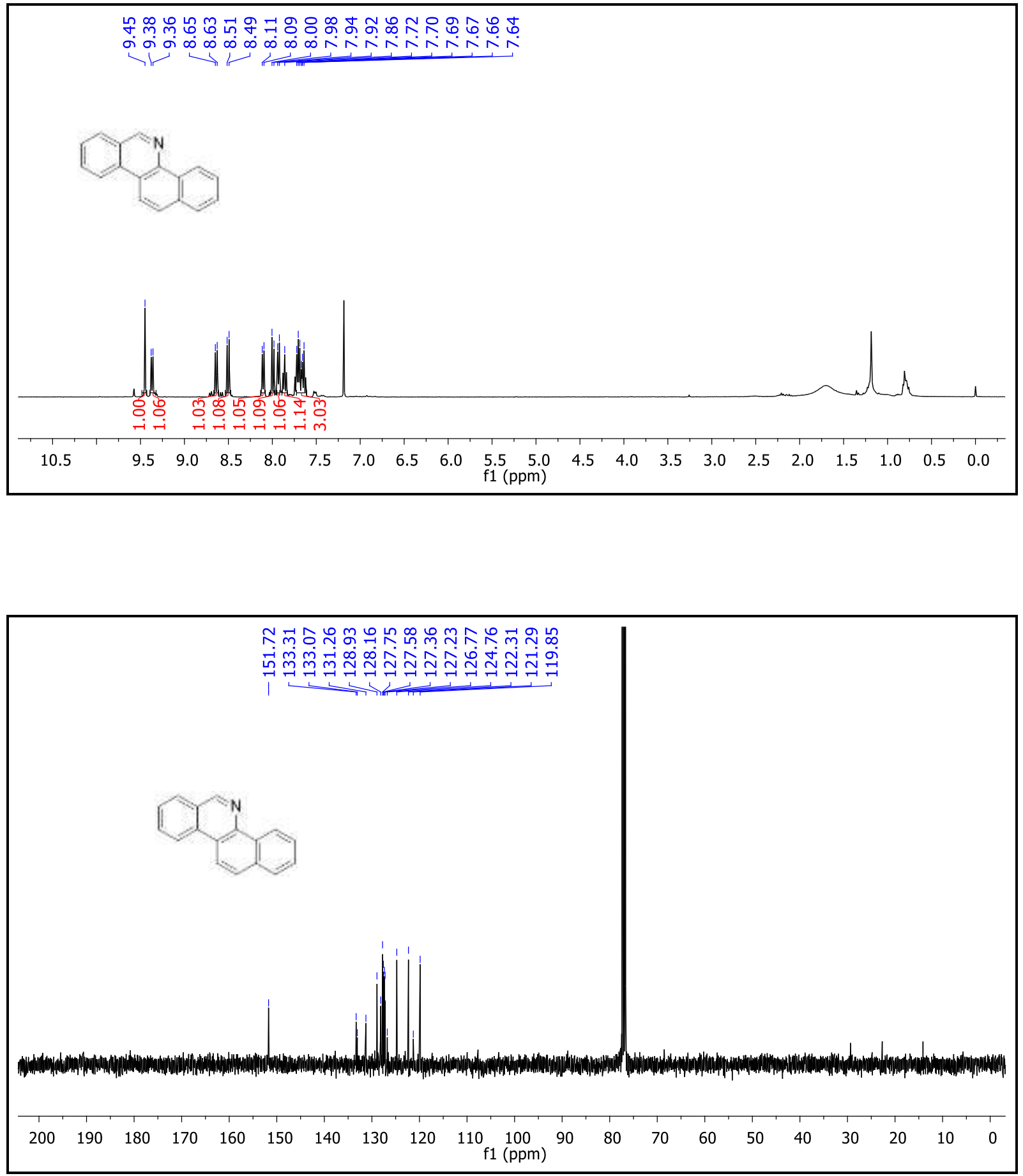
DEPT (135) NMR Spectrum of Compound $\mathbf{5 a}\left(\mathrm{CDCl}_{3}, 101 \mathrm{MHz}\right)$.

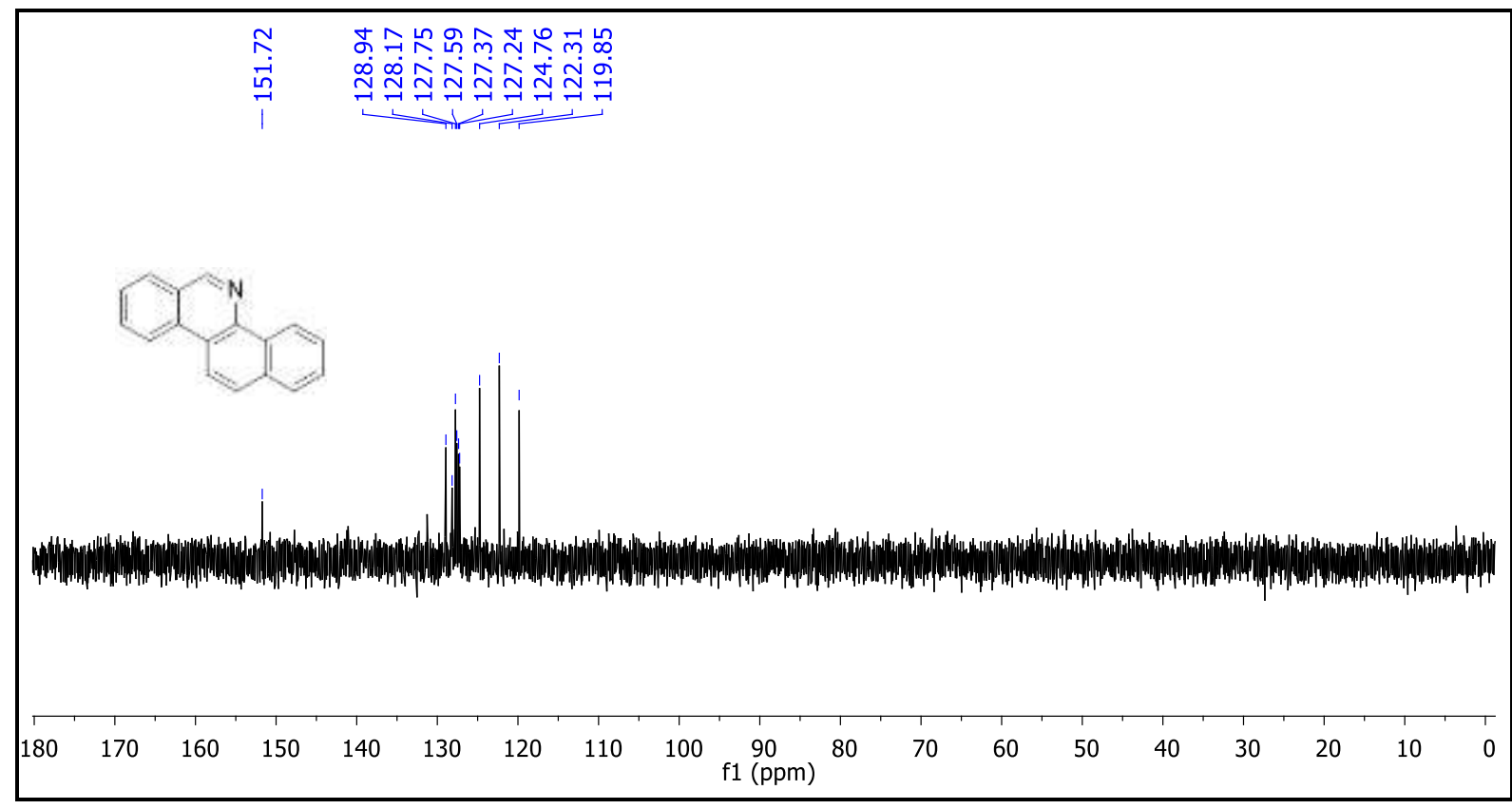


${ }^{1} \mathrm{H}$ and ${ }^{13} \mathrm{C}\left\{{ }^{1} \mathrm{H}\right\}$ NMR Spectra of Compound $\mathbf{5 b}\left(\mathrm{CDCl}_{3}, 400 \mathrm{MHz}\right)$.
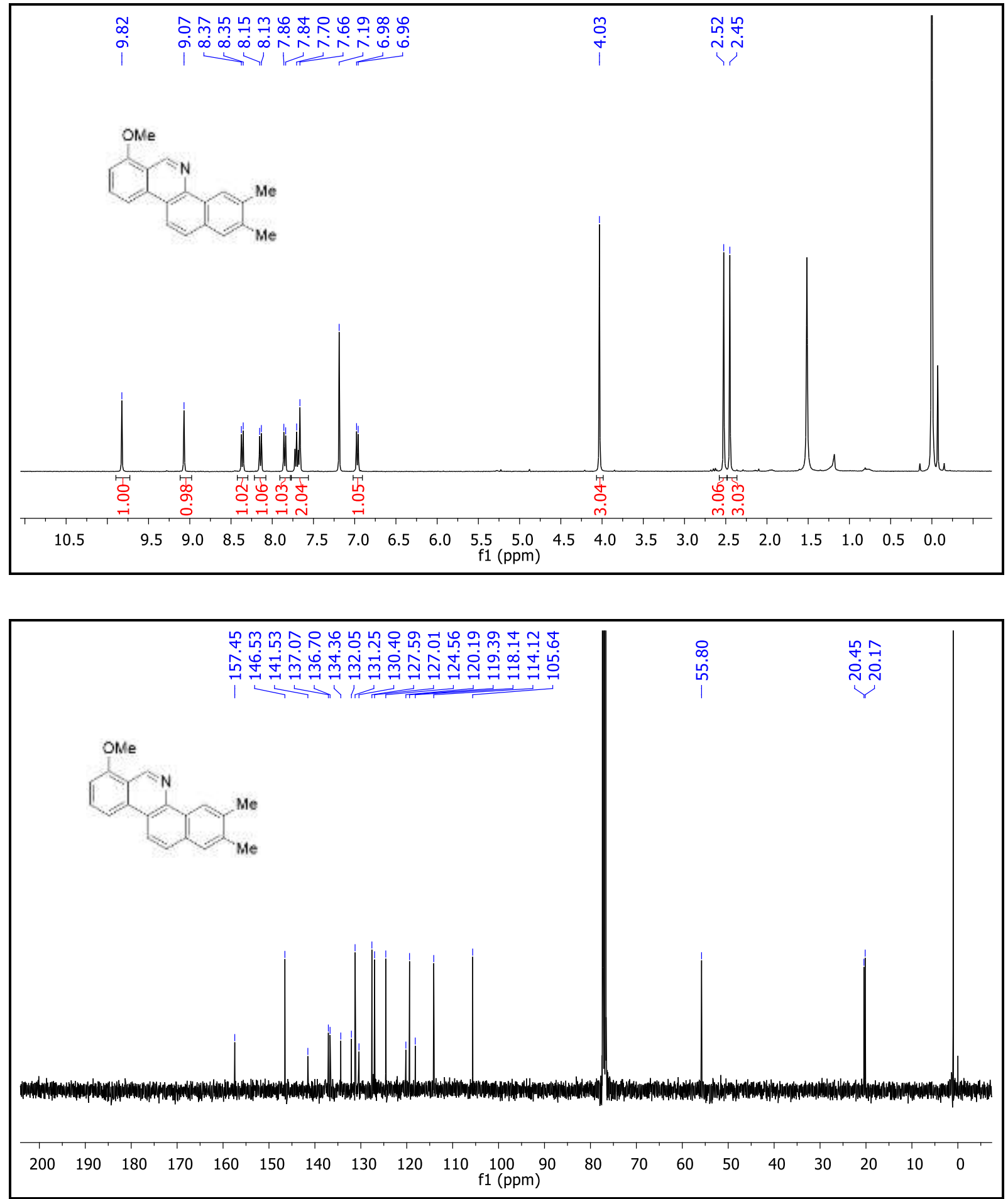
DEPT (135) NMR Spectrum of Compound $\mathbf{5 b}\left(\mathrm{CDCl}_{3}, 101 \mathrm{MHz}\right)$.

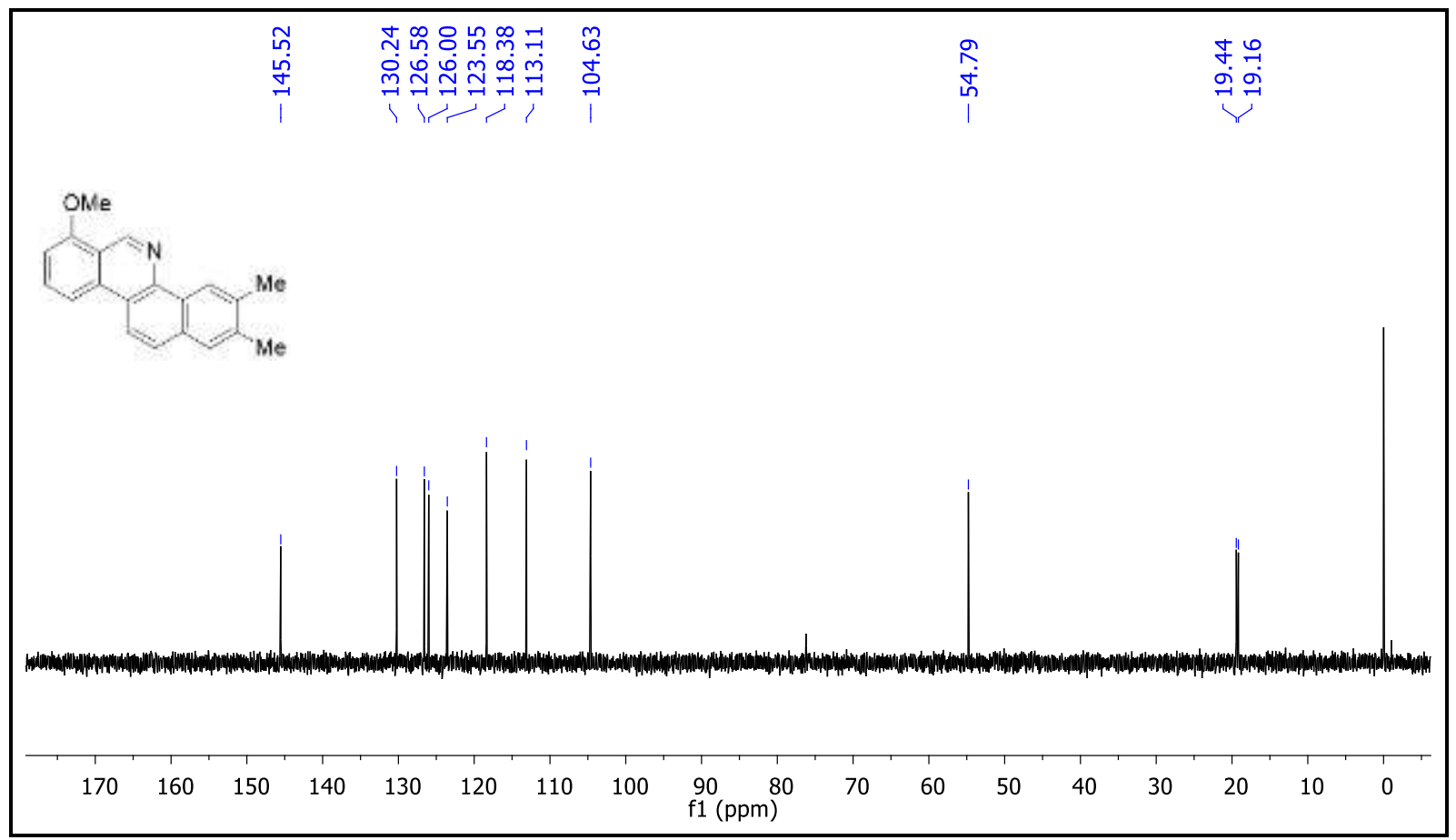


${ }^{1} \mathrm{H}$ and ${ }^{13} \mathrm{C}\left\{{ }^{1} \mathrm{H}\right\}$ NMR Spectra of Compound $\mathbf{5 c}\left(\mathrm{CDCl}_{3}, 400 \mathrm{MHz}\right)$.
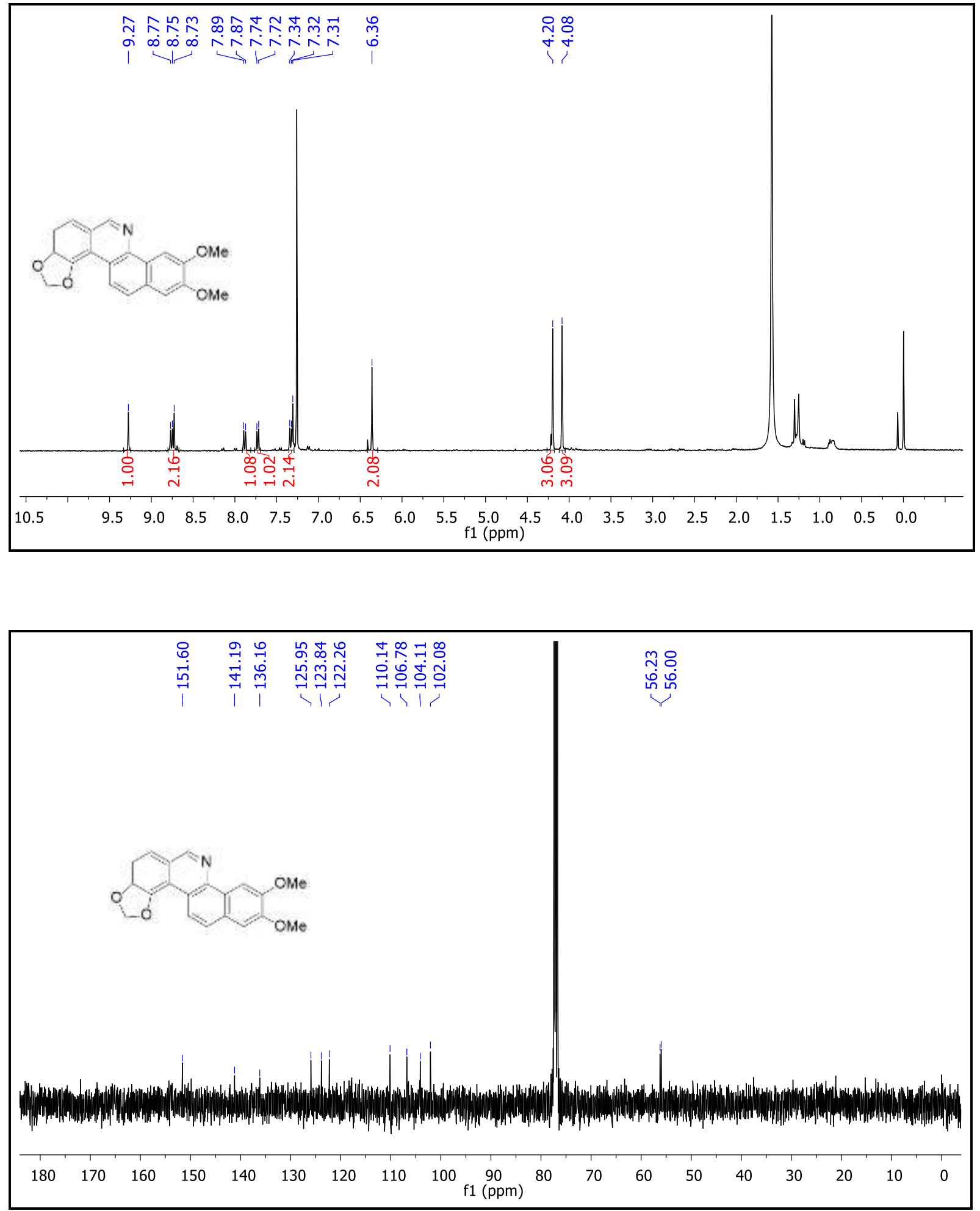
DEPT (135) NMR Spectrum of Compound $\mathbf{5 c}\left(\mathrm{CDCl}_{3}, 101 \mathrm{MHz}\right)$.

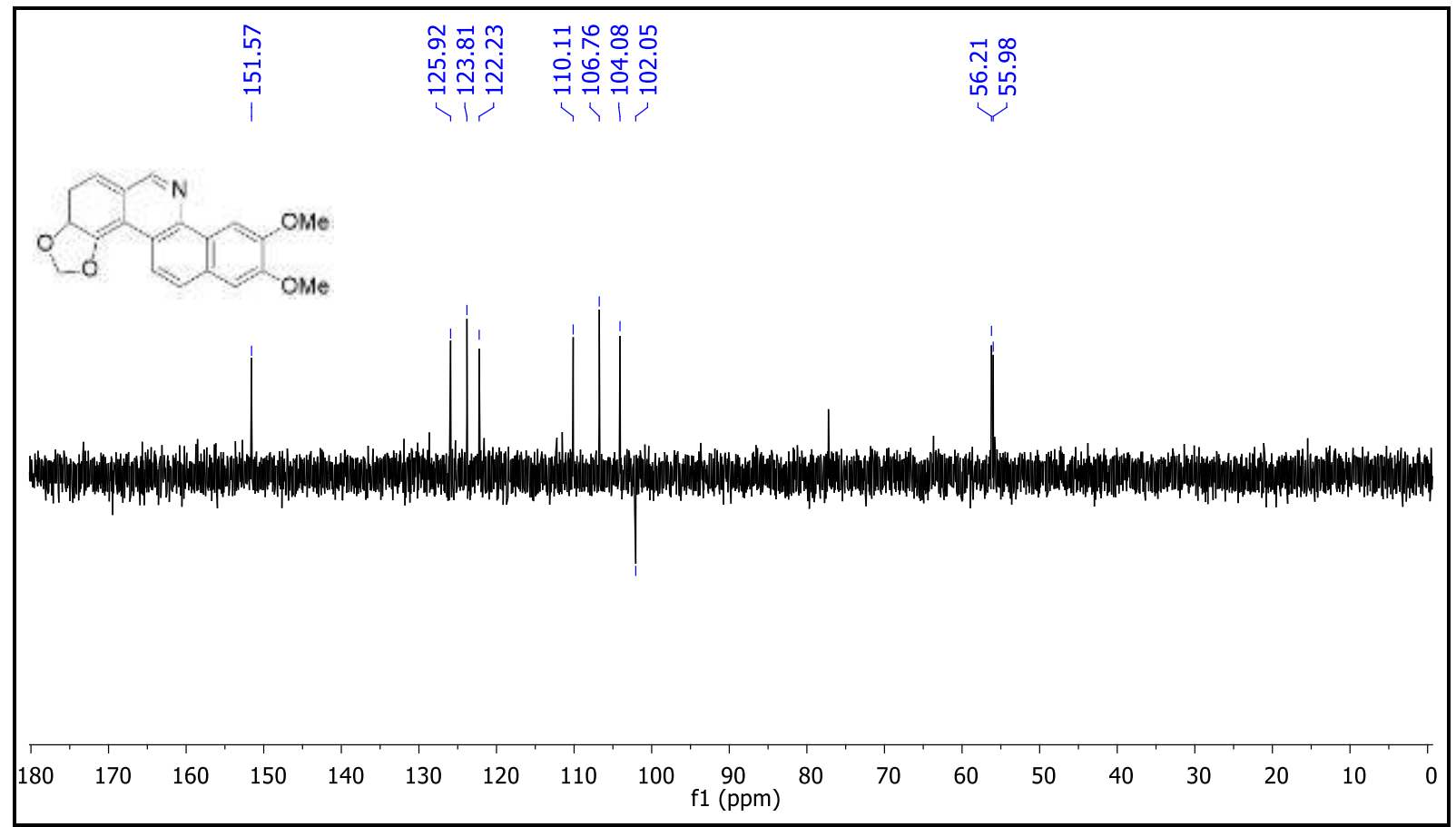


${ }^{1} \mathrm{H}$ and ${ }^{13} \mathrm{C}\left\{{ }^{1} \mathrm{H}\right\}$ NMR Spectra of Compound $\mathbf{5 d}\left(\mathrm{CDCl}_{3}, 500 \mathrm{MHz}\right)$.
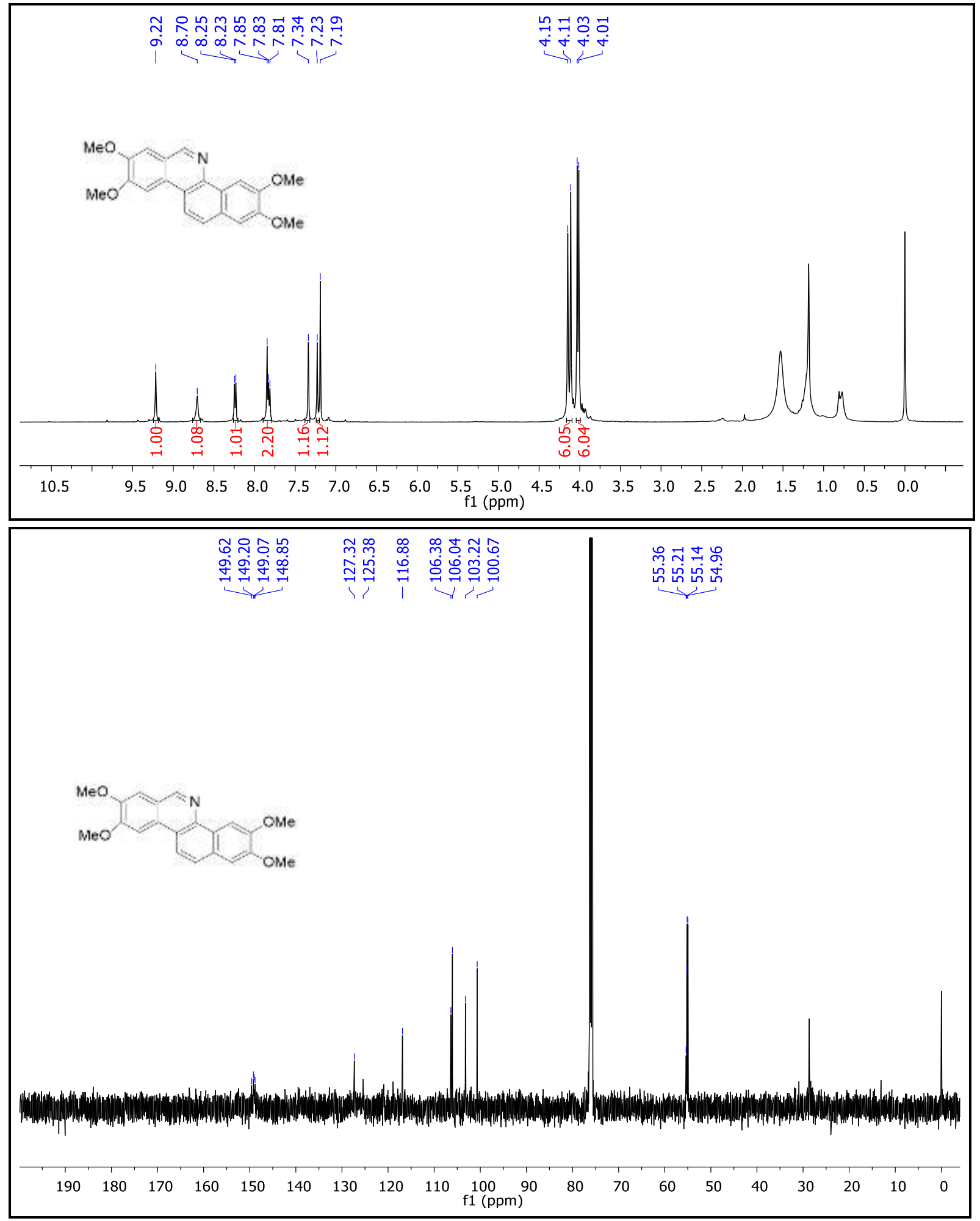
DEPT (135) NMR Spectrum of Compound $\mathbf{5 d}\left(\mathrm{CDCl}_{3}, 126 \mathrm{MHz}\right)$.

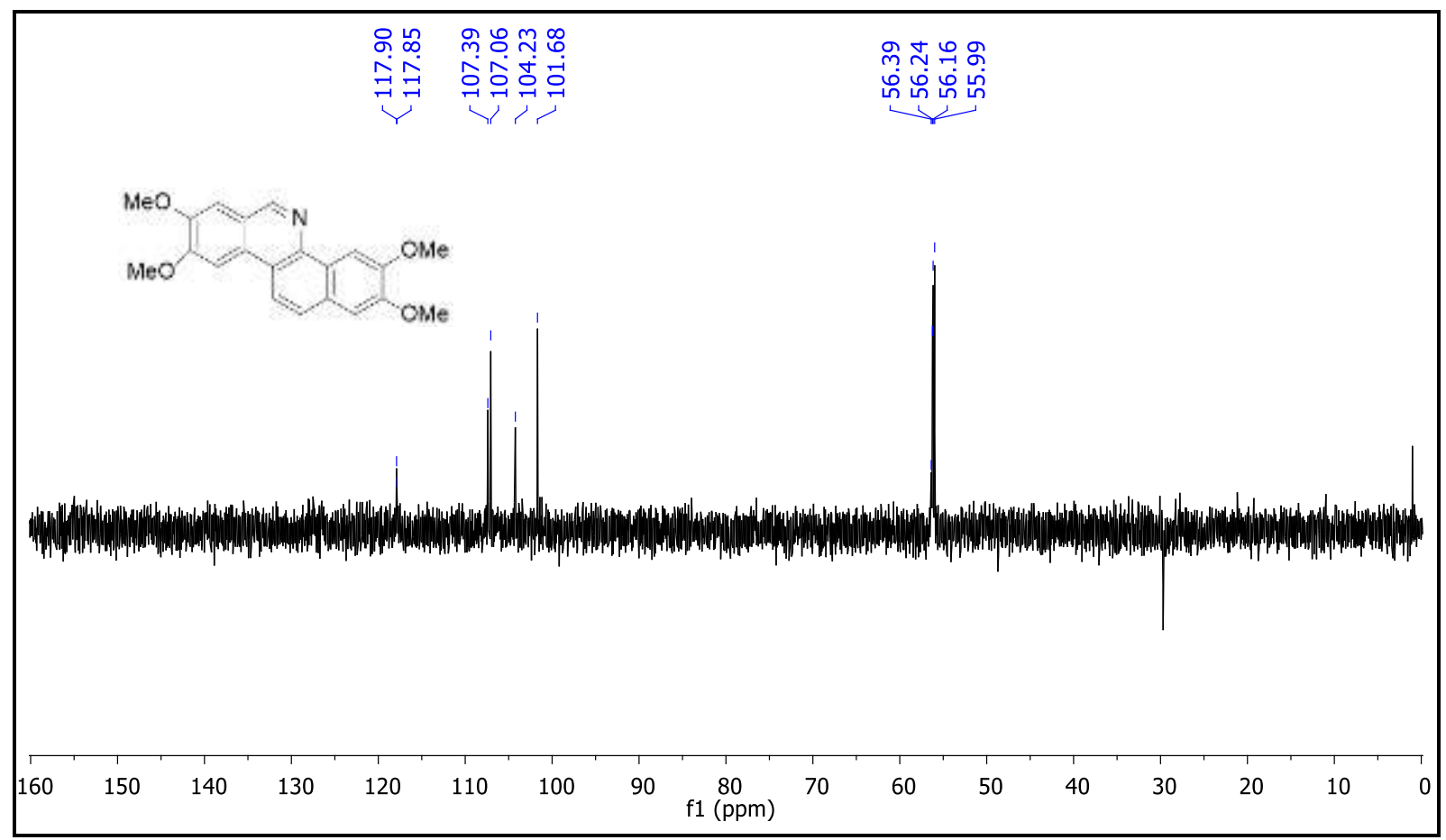


${ }^{1} \mathrm{H}$ and ${ }^{13} \mathrm{C}\left\{{ }^{1} \mathrm{H}\right\}$ NMR Spectra of Compound 5e (DMSO- $\left.d_{6}, 500 \mathrm{MHz}\right)$.
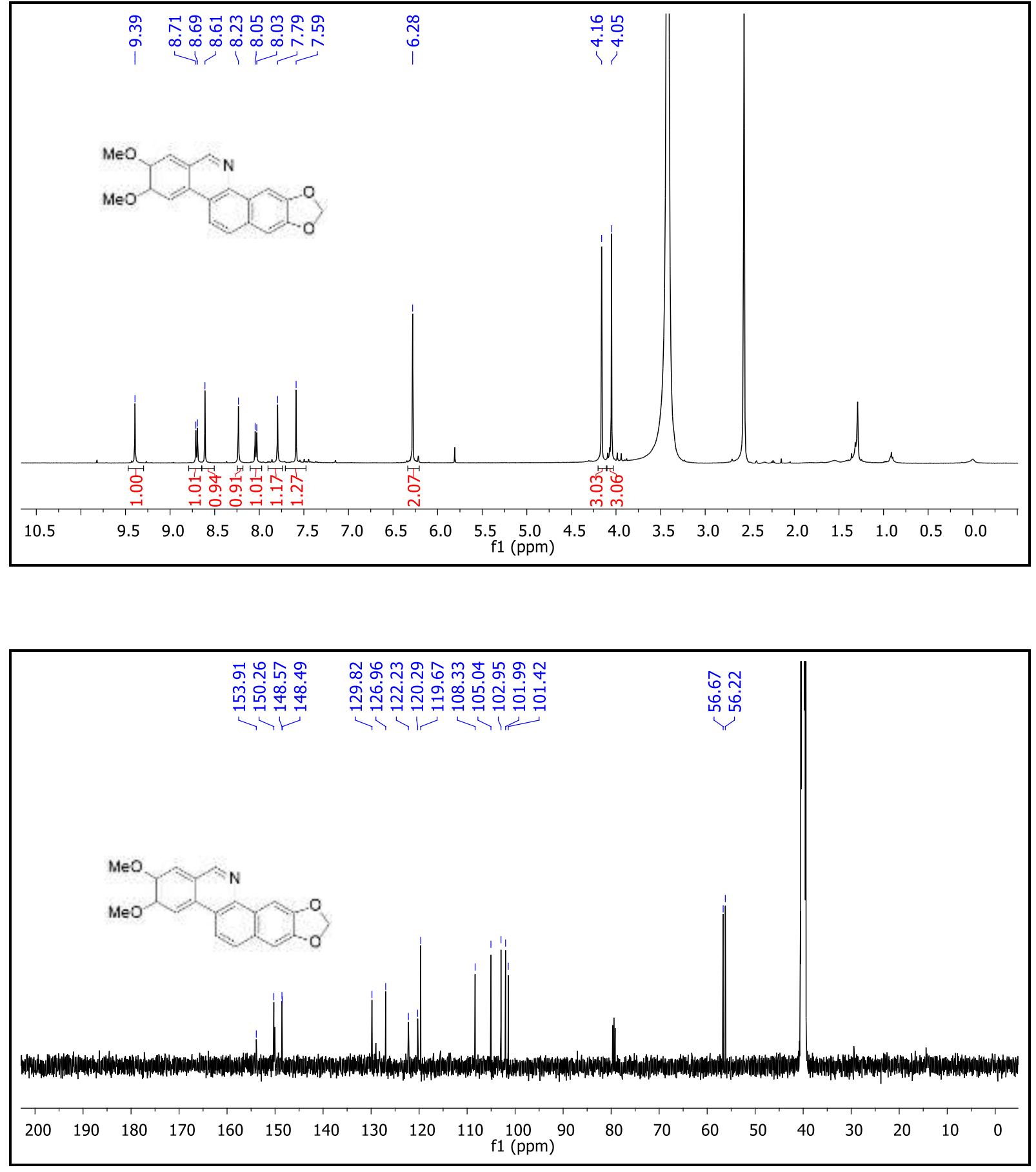
DEPT (135) NMR Spectrum of Compound 5e (DMSO- $d_{6}, 126 \mathrm{MHz}$ ).

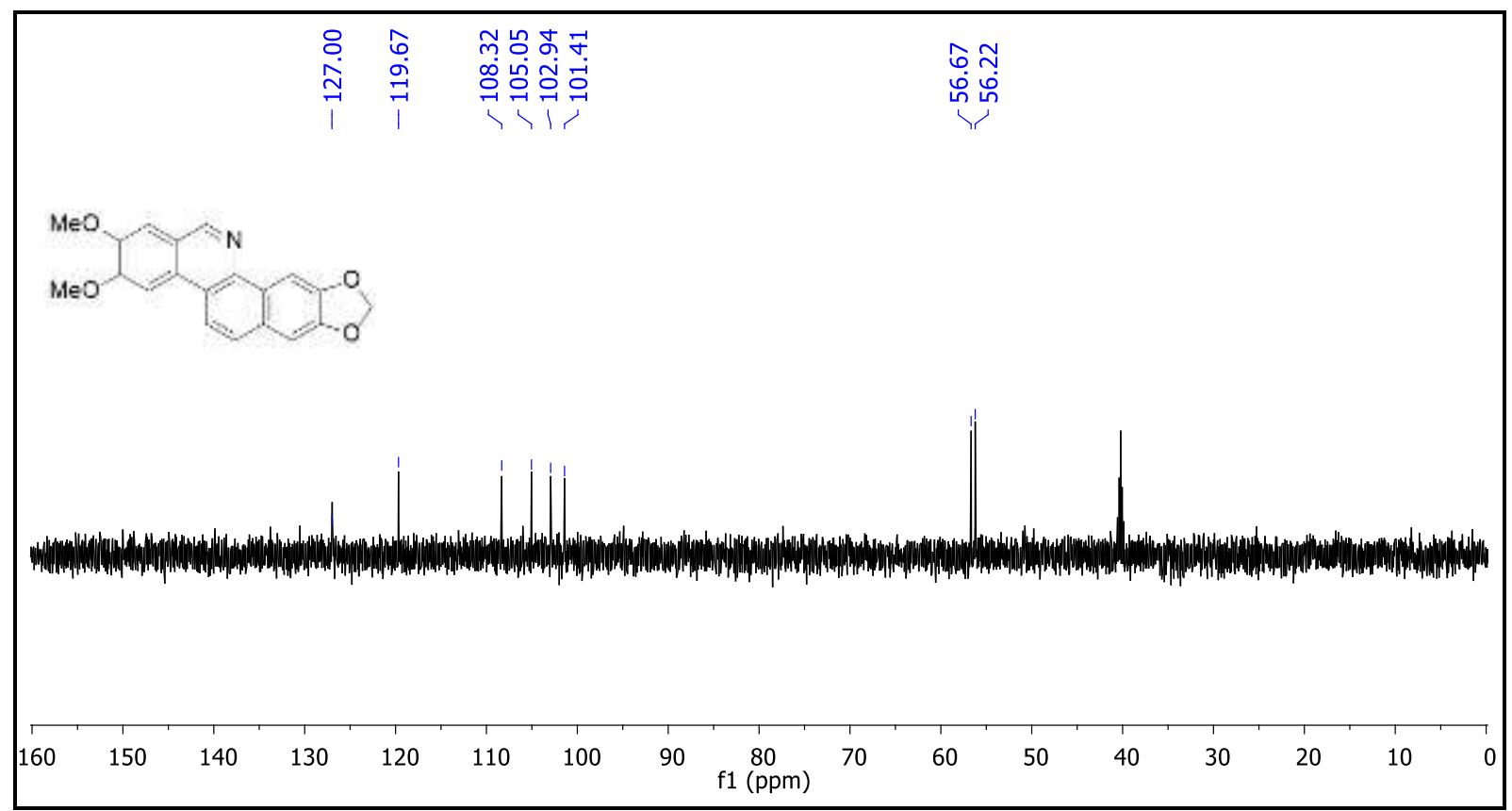


${ }^{1} \mathrm{H}$ and ${ }^{13} \mathrm{C}\left\{{ }^{1} \mathrm{H}\right\}$ NMR Spectra of Compound $\mathbf{5 f}$ (DMSO- $d_{6}, 400 \mathrm{MHz}$ ).
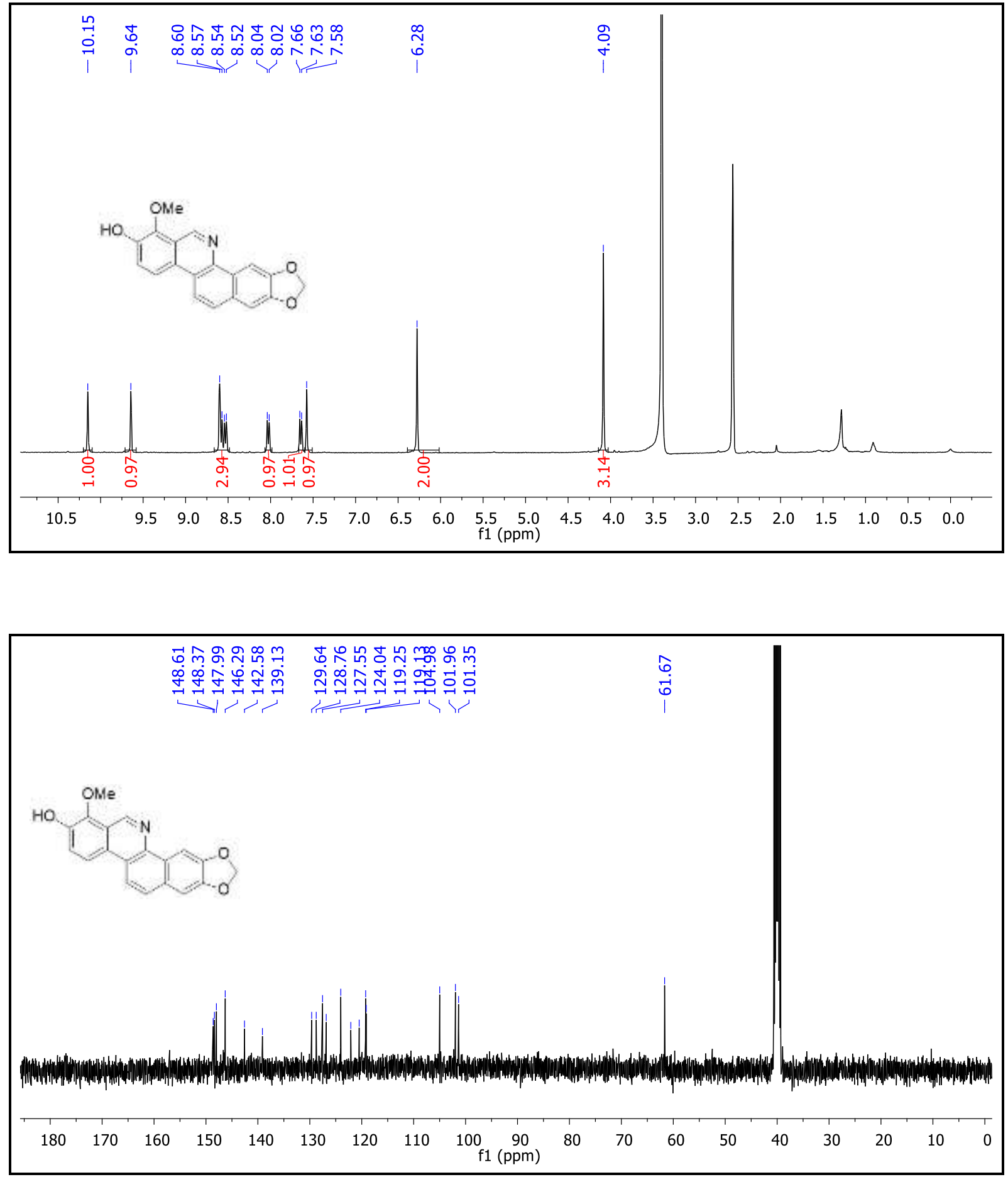
DEPT (135) NMR Spectrum of Compound $\mathbf{5 f}$ (DMSO- $d_{6}, 101 \mathrm{MHz}$ ).

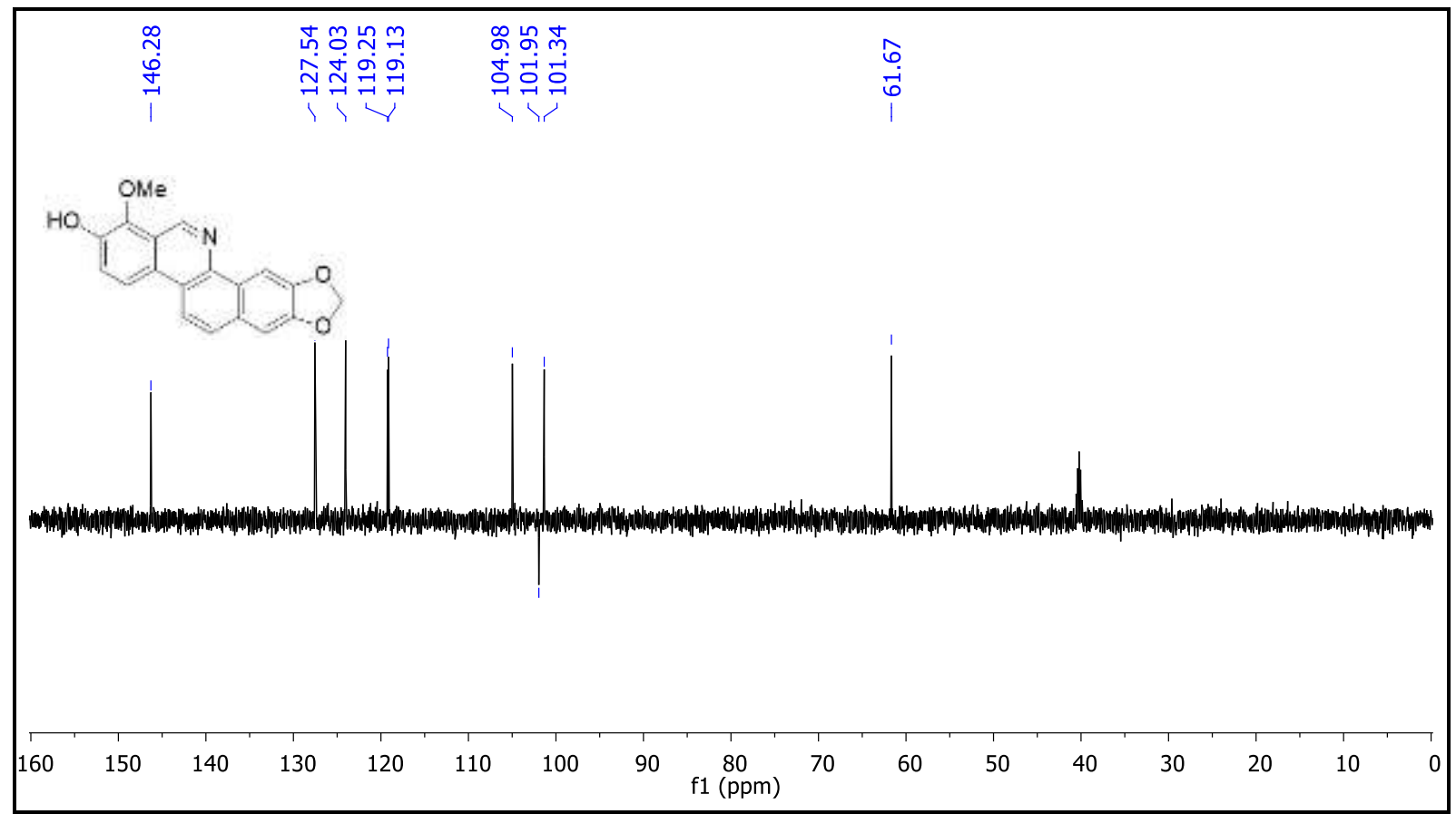


${ }^{1} \mathrm{H}$ and ${ }^{13} \mathrm{C}\left\{{ }^{1} \mathrm{H}\right\}$ NMR Spectra of Compound $5 \mathrm{~g}$ (DMSO- $d_{6}, 400 \mathrm{MHz}$ ).
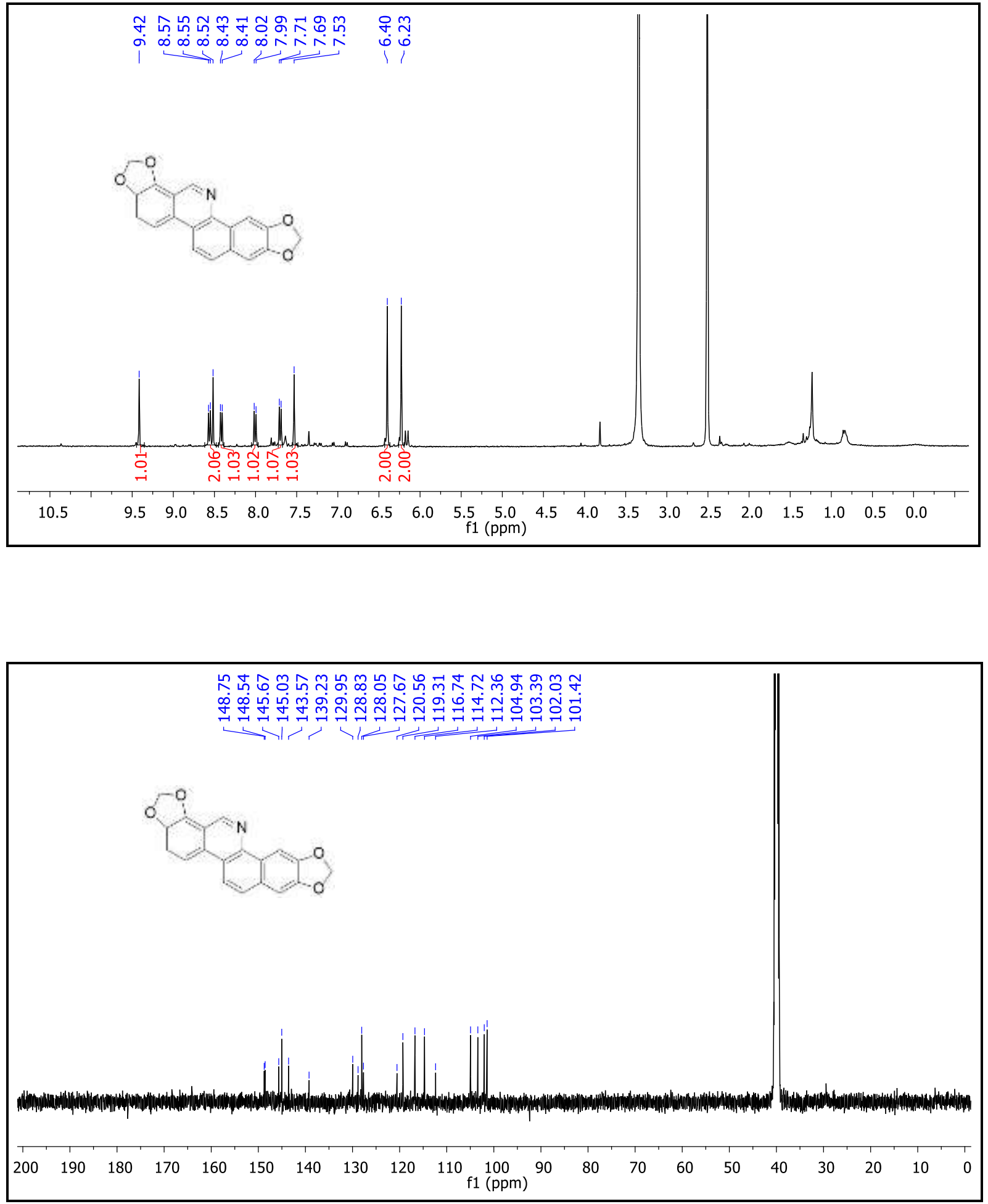
DEPT (135) NMR Spectrum of Compound 5g (DMSO- $d_{6}, 126 \mathrm{MHz}$ ).

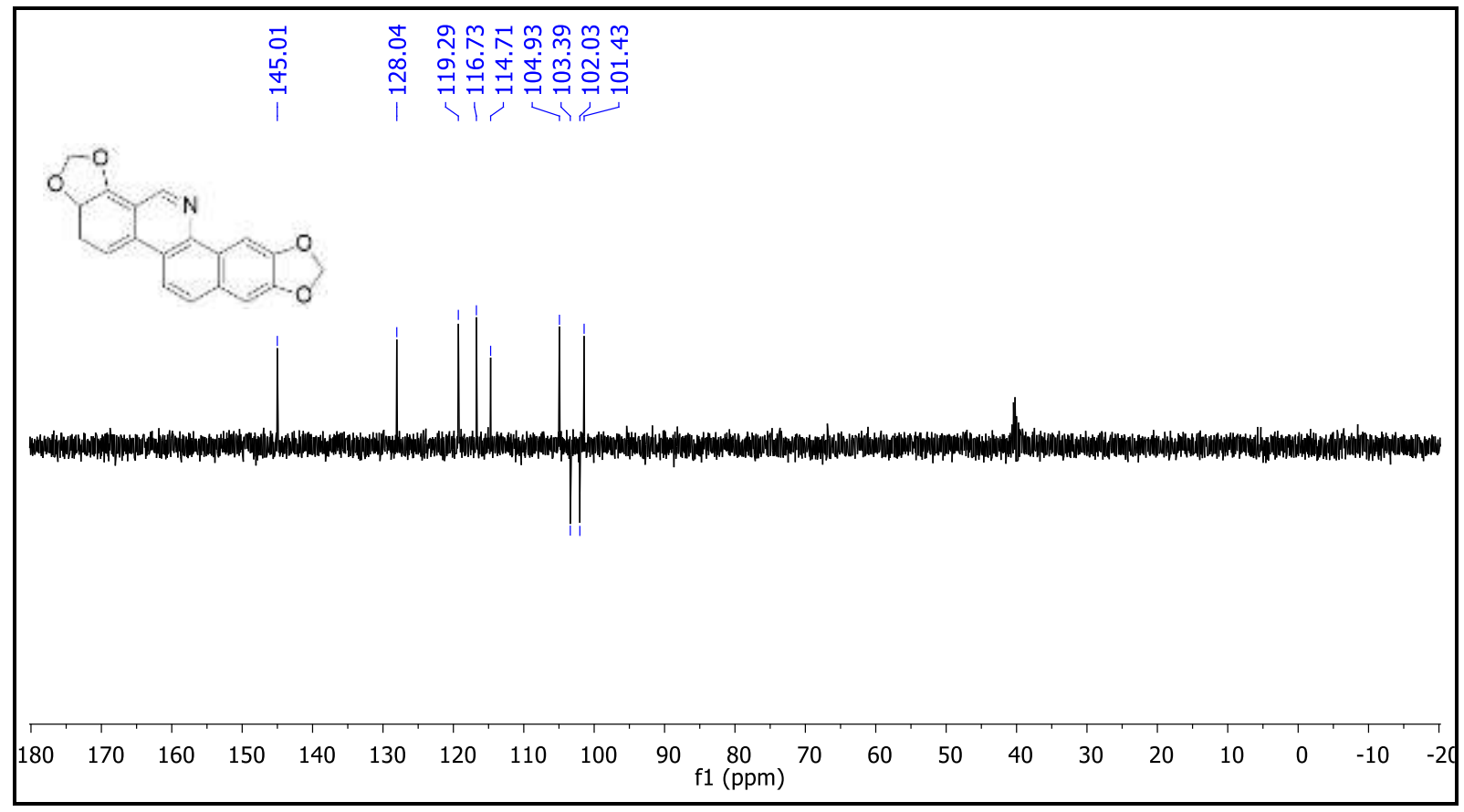


${ }^{1} \mathrm{H}$ and ${ }^{13} \mathrm{C}\left\{{ }^{1} \mathrm{H}\right\}$ NMR Spectra of Compound $\mathbf{5 h}\left(\mathrm{CDCl}_{3}, 500 \mathrm{MHz}\right)$.
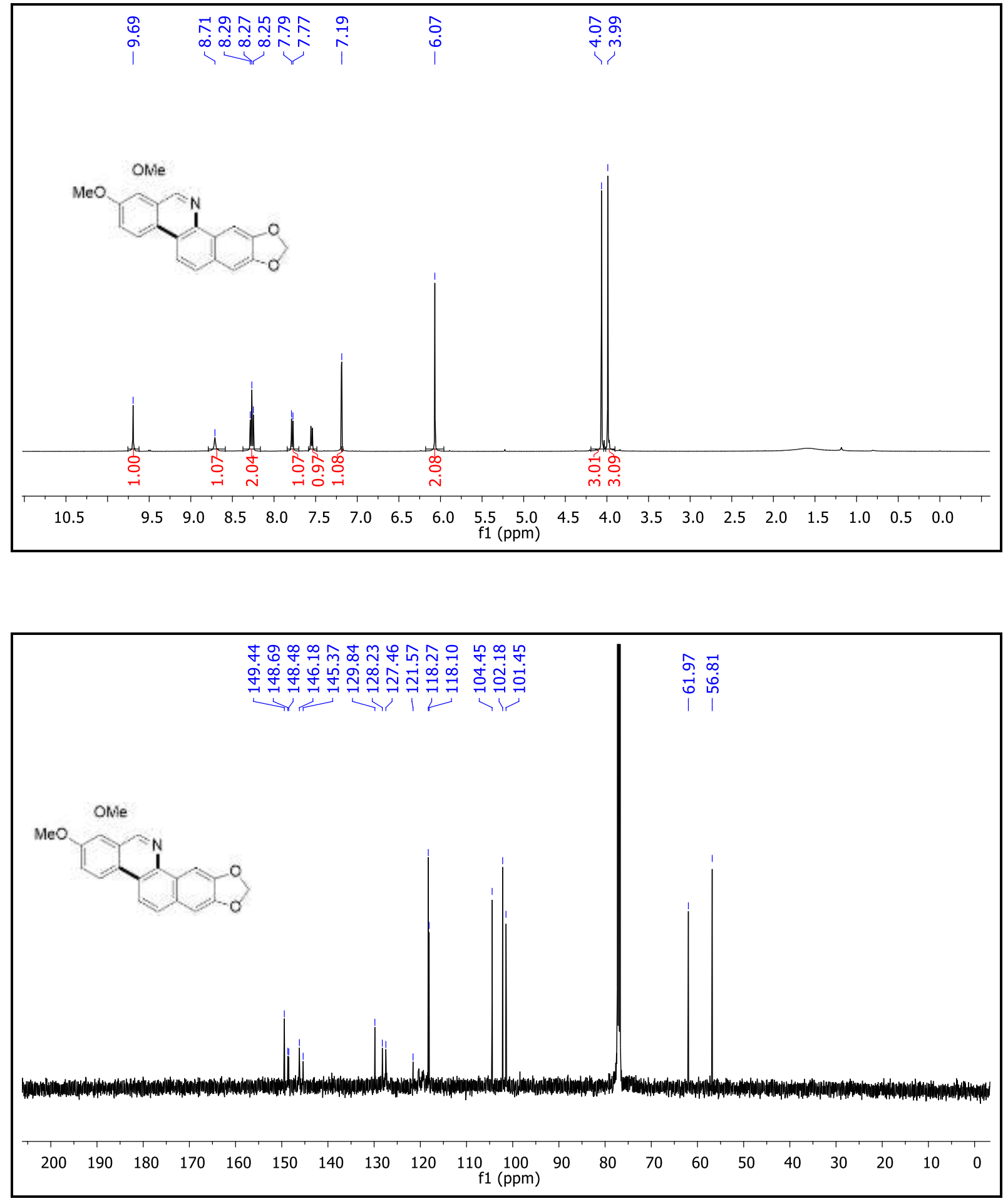
DEPT (135) NMR Spectrum of Compound $\mathbf{5 h}\left(\mathrm{CDCl}_{3}, 126 \mathrm{MHz}\right)$.

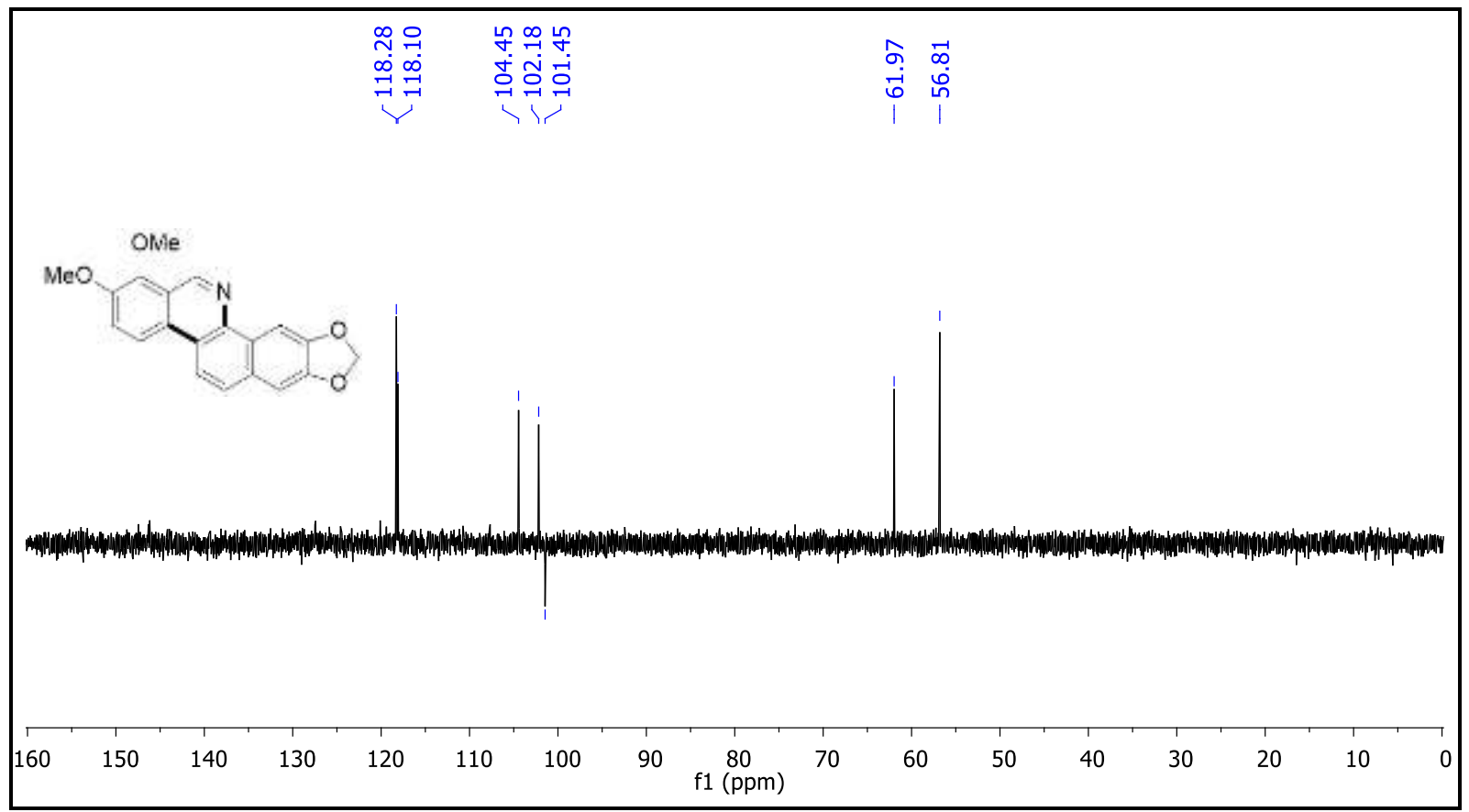

